Florida International University

FIU Digital Commons

$6-26-2020$

\title{
Boron Nitride Nanotube based Lightweight Metal Matrix Composites: Microstructure Engineering and Stress-Transfer Mechanics
}

Pranjal Nautiyal

Florida International University, pnaut001@fiu.edu

Follow this and additional works at: https://digitalcommons.fiu.edu/etd

Part of the Manufacturing Commons, Metallurgy Commons, and the Structural Materials Commons

\section{Recommended Citation}

Nautiyal, Pranjal, "Boron Nitride Nanotube based Lightweight Metal Matrix Composites: Microstructure Engineering and Stress-Transfer Mechanics" (2020). FIU Electronic Theses and Dissertations. 4513. https://digitalcommons.fiu.edu/etd/4513

This work is brought to you for free and open access by the University Graduate School at FIU Digital Commons. It has been accepted for inclusion in FIU Electronic Theses and Dissertations by an authorized administrator of FIU Digital Commons. For more information, please contact dcc@fiu.edu. 


\section{FLORIDA INTERNATIONAL UNIVERSITY}

Miami, Florida

BORON NITRIDE NANOTUBE BASED LIGHTWEIGHT METAL MATRIX

COMPOSITES:

MICROSTRUCTURE ENGINEERING AND STRESS-TRANSFER MECHANICS

A dissertation submitted in partial fulfillment of

the requirements for the degree of

DOCTOR OF PHILOSOPHY

in

MATERIALS SCIENCE AND ENGINEERING

by

Pranjal Nautiyal 
To: Dean John L. Volakis

College of Engineering and Computing

This dissertation, written by Pranjal Nautiyal, and entitled Boron Nitride Nanotube based Lightweight Metal Matrix Composites: Microstructure Engineering and Stress-Transfer Mechanics, having been approved in respect to style and intellectual content, is referred to you for judgment.

We have read this dissertation and recommend that it be approved.

$\begin{array}{r}\text { Zhe Cheng } \\ \hline \text { Seung Jae Lee } \\ \hline \text { Benjamin Boesl, Co-Major Professor } \\ \hline \text { Arvind Agarwal, Co-Major Professor }\end{array}$

Date of Defense: June 26, 2020

The dissertation of Pranjal Nautiyal is approved.

Dean John L. Volakis
College of Engineering and Computing

Andrés G. Gill

Vice President for Research and Economic Development and Dean of the University Graduate School

Florida International University, 2020 
(C) Copyright 2020 by Pranjal Nautiyal

All rights reserved. 


\section{DEDICATION}

I dedicate this dissertation to my parents, family, teachers and mentors, who have shaped me into the person I am today. 


\section{ACKNOWLDGMENTS}

I want to express my sincere gratitude to my major professor, Dr. Arvind Agarwal, for playing a critical role in the planning, execution and conclusion of this research. I had the privilege of working with Prof. Agarwal as an undergraduate researcher first, and my decision to pursue Ph.D. was based on his encouragement and support. When I look back, that was the best decision I have taken in my life so far. I cannot thank Prof. Agarwal enough for the countless hours he has spent reviewing my progress, scrutinizing my results, providing timely feedback and making sure my work is rapidly published. I am grateful for all the opportunities he has provided me to excel professionally, including access to cutting edge research facilities, opportunities to present my work in numerous international forums, opportunity to write a book, participate in proposal writing activities, and collaborations with world-class researchers on brilliant research projects. Beyond a mentor, Dr. Agarwal is a cheerleader who pushes me to perform better every single day and he lifts me up every time I hit obstacles or face disappointments.

I want to thank my co-advisor, Dr. Benjamin Boesl, for facilitating the successful completion of my Ph.D. project through his guidance and expertise on the mechanics of composites. Dr. Boesl mentored me as I grasped and worked in the area of in-situ mechanical characterization, which has become a centerpiece of my research activities over the last 4 years. Dr. Boesl was kind and patient to spend time with me in the laboratory, providing feedback with my experimental plans and reviewing the manuscripts published out of this dissertation project. In the last 5 years, we have forged a pleasant and productive professional relationship. 
I sincerely thank Drs. Seung Jae Lee and Zhe Cheng for serving in my committee. Their yearly feedback on my research was helpful to ensure I meet my dissertation objectives in a timely manner. I'm grateful for their time, efforts, and responsiveness.

I have collaborated with several of my colleagues in the Plasma Forming Group. I would like to acknowledge Dr. Archana Loganathan, Dr. Cheng Zhang, Dr. Chris Rudolf, Jenniffer Bustillos, Xiaolong Lu, Noemie Denis, and Tyler Dolmetsch. Their contributions were instrumental in meeting many of the key dissertation objectives and publication of several journal articles related to this project. I gained valuable knowledge and insights working with them.

A significant portion of my research is carried out in the Advanced Materials Engineering Research Institute (AMERI) at FIU. I would like to particularly thank Dr. Alex Franco, an AMERI staff and research assistant professor, for spending long days training me, aiding in electron microscope characterizations and facilitating equipment troubleshooting. Some of the central segments of my research would not have been completed without Dr. Franco's assistance. I would also like to acknowledge former AMERI staff members, Drs. Yusuf Emirov and Shravana Katakam, for their assistance with focused ion beam machining and transmission electron microscopy.

I would like to mention the outstanding contributions from our collaborators at the University of Central Florida, Prof. Sudipta Seal, Dr. Tamil S. Sakthivel and Dr. Ankur Gupta (now with Persimmon Technologies Corporation), who carried out high-resolution transmission electron microscopy and X-ray photoelectron spectroscopy of nanocomposite specimens. Their expertise was helpful in deciphering the complex microstructures of the 
composites studied herein. I also thank Prof. Chunlei Wang (in my department) and Dr. Richa Agrawal (a former FIU graduate student) for assisting us with Fourier transform infrared spectroscopy.

I would like to acknowledge the financial support received from FIU Graduate School through the Presidential Fellowship and Dissertation Year Fellowship awards. I also thank the Office of Naval Research grant (N00014-17-12563), the Army Research Laboratory grant (W911NF15-2-0026), and the National Science Foundation-Engineering Research Centers Program (CELL-MET ERC, NSF Cooperative Agreement No. EEC1647837) for research assistantship support and funding research supplies used to carry out the experiments.

I'm thankful to Dr. Roy Whitney of BNNT, LLC. for providing nanotube samples useful to meet some of the central dissertation objectives. BNNT, LLC. is our trusted source of high-quality nanotubes, which is fundamental to the success of this project.

I also express my gratitude to the current and former staff members of the Mechanical and Materials Engineering and AMERI for their assistance with logistics and administrative matters. I would like to mention Mabel Fernandez, Tiziana Leoni, and Beatriz Morillo, who would go out of their way to provide the best possible assistance with my assistantship contracts, research purchases, and work-related travels.

No man is an island, and I'm highly appreciative of all my current and former colleagues in the Plasma Forming Group for their support and encouragement. I have developed profound friendships in the last 5 years at FIU. I wish to thank Leslie Embrey, Melania Antillon, Archana Loganthan, Richa Agrawal, Tony Thomas, Jenniffer Bustillos, 
Cheng Zhang, Kazue Orisaka, Laura Reyes, Sadhana Bhusal, Luiza Fontoura, Tanaji Paul, Giuseppe Bianco, Cat Young and Sadegh Behdad for some of the fondest memories. Leslie and Richa have become my family in the United States, and I treasure their friendship.

Most importantly, I express my deepest gratitude to my loving family: my parents, my aunts and uncles, my siblings, and my grandparents. I derive my humanity and strength of character from them. My father, in particular, is a role model for me, and I always strive to emulate him in leading my life with integrity, courage, and conviction. 


\author{
ABSTRACT OF THE DISSERTATION \\ BORON NITRIDE NANOTUBE BASED LIGHTWEIGHT METAL MATRIX \\ COMPOSITES: \\ MICROSTRUCTURE ENGINEERING AND STRESS-TRANSFER MECHANICS \\ by
}

Pranjal Nautiyal

Florida International University, 2020

Miami, Florida

Professor Arvind Agarwal, Co-Major Professor

Professor Benjamin Boesl, Co-Major Professor

Lightweight metals, such as Aluminum, Magnesium and Titanium, are receiving widespread attention for manufacturing agile structures. However, the mechanical strength of these metals and their alloys fall short of structural steels, curtailing their applicability in engineering applications where superior load-bearing ability is required. There is a need to effectively augment the deformation- and failure-resistance of these metals without compromising their density advantage.

This dissertation explores the mechanical reinforcement of the aforementioned lightweight metal matrices by utilizing Boron Nitride Nanotube (BNNT), a 1D nanomaterial with extraordinary mechanical properties. The nanotubes are found to resist thermo-oxidative transformations up to $\sim 750^{\circ} \mathrm{C}$, establishing their suitability for engineering metal matrix composites. Al-BNNT composites are fabricated by three classes of scalable processing approaches: powder metallurgy, solidification and plasma spray additive manufacturing. These processing techniques unravel metal-nanotube interactions 
in a vast processing space, such as state of metal (solid versus liquid), the timescale of interactions $\left(10^{-3}\right.$ to $\left.10^{+3} \mathrm{~s}\right)$, range of temperatures $\left(10^{2}\right.$ to $\left.10^{3 \circ} \mathrm{C}\right)$ and pressures $\left(10^{-1}\right.$ to $10^{+1}$ $\mathrm{MPa}$ ). Limited interfacial reactions between $\mathrm{Al}$ and BNNT are observed, which improve wetting, capture, bonding and stress-transfer in the composites. Consequently, remarkable mechanical reinforcement is achieved, with $\sim 400 \%$ improvement in tensile strength, an order of magnitude jump in hardness, and up to two-fold enhancement of elastic modulus. Nanofiller assisted reinforcement of Magnesium alloys is challenging because of their low plasticity. Therefore, an architected, layered composite of AZ31 Mg alloy and BNNT is engineered to minimize embrittlement. In-situ double cantilever testing reveals effective crack bridging by BNNT, facilitated by reactive interface bonding.

Inspired by the importance of interphases, this work investigates the correlation between deformation mechanisms and chemical make-up of the composites. Ti-6Al-4V alloy is reinforced with BNNT and processed at two different temperatures (750 and $950^{\circ} \mathrm{C}$ ) to induce varying degrees of interfacial reactions. Real-time imaging of deformation, in conjunction with high-resolution chemical mapping, provides insights into the synergistic strengthening of the alloy due to interphases and BNNT present in the microstructure. The holistic understanding of microstructure evolution and mechanics of stress-transfer advanced by this dissertation will be helpful for engineering lightweight BNNT-MMCs with superior mechanical performance. 


\section{TABLE OF CONTENTS}

$\begin{array}{ll}\text { CHAPTER } & \text { PAGE }\end{array}$

1. INTRODUCTION 1

1.1. Lightweight Metal Matrix Composites 1

1.2. Boron Nitride Nanotube as a Structural Reinforcement 4

1.3. Challenges and Limitations in Current Understanding 5

1.3.1. Nanotube Morphology and Purity 5

1.3.2. Metal Matrix Composite Processing 6

1.3.3. Challenges with Mechanical Properties 7

1.4. Objectives of the Research 9

$\begin{array}{ll}\text { References } & 13\end{array}$

2. A CRITICAL REVIEW OF THE STATE-OF-THE-ART 15

2.1. Thermal Stability of Boron Nitride Nanotubes 15

2.2. Mechanical Properties of Boron Nitride Nanotubes 18

2.2.1. Tensile Behavior 18

2.2.2. Bending Behavior 19

2.2.3. Radial Mechanical Properties $\quad 21$

2.2.4. Torsional Mechanical Properties 23

2.3. Processing of Boron Nitride Nanotube-Metal Matrix Composites 25

2.3.1. Synthesis of Composite Precursor 25

2.3.2. Manufacturing Approaches $\quad 27$

2.3.3. Critique 32

2.4. Microstructure of Boron Nitride Nanotube-Metal Matrix Composites 35

2.4.1. Study of Nanotube Integration and Dispersion in the Matrix 35

2.4.2. Critique 45

2.5. Mechanical Properties of Boron Nitride Nanotube-Metal Matrix

Composites 48

2.5.1. Microhardness 48

2.5.2. Compression Strength 51 
2.6. In-situ Investigation of Deformation Mechanisms in Boron Nitride Nanotube-Metal Matrix Composites 60

2.6.1. In-situ Tensile Deformation 60

2.6.2. In-situ Bending and Fracture 63

2.6.3. Critique 64

2.7. Missing Gaps in Current Understanding: Some Critical Questions 65

$\begin{array}{ll}\text { References } & 67\end{array}$

3. THEORETICAL FRAMEWORK 71

3.1. Intertube Interactions 71

3.1.1. Nanoparticle Agglomeration $\quad 71$

3.1.2. Implication for Metal Matrix Composite Processing 73

3.1.3. Approach Adopted in this Work: Physical-Chemical

Dispersion to Address Clustering 74

3.2. Nanotube Orientation vis-à-vis Mechanical Properties of Composite 75

3.2.1. Orientation-Sensitive Shear Lag Model 75

3.2.2. Application to this Work: Engineering Percolated

Composite Microstructure $\quad 76$

3.3. Nanocomposite Interface Mechanics 77

3.3.1. Matrix-Filler Interphases $\quad 77$

3.3.2. Application to this Work: Chemical Tuning of Interfacial Stress-Transfer $\quad 78$

3.4. Solidification Front-Nanotube Interactions 80

3.4.1. Nanoparticle Capture-Repulsion Dichotomy $\quad 80$

3.4.2. Application to this Work: Strategies for Solidification Processing 83

3.5. Strength-Ductility Tradeoff 85

3.5.2. Dislocation Obstruction $\quad 85$ 
3.5.2. Approach Adopted in this Work: Architected Nanocomposites 86

3.6. Discrete-Continuum Duality 88

3.6.1. Hierarchical Microstructure, Interface Complexity and Size-Effect $\quad 88$

3.6.2. Approach Adopted in this Work: Multi-scale and In-situ Mechanical Investigation $\quad 89$

3.7. Takeaway: Theoretical Framework to Meet Dissertation Objectives 90

References 91

4. MATERIALS, EXPERIMENTAL METHODOLOGIES AND ANALYSIS TECHNIQUES 94

4.1. Boron Nitride Nanotube Precursor 94

4.1.1. Puffy Balls 94

4.1.2. Dispersion of Nanotubes 96

4.1.3. Buckypaper 97

4.2. Processing Approaches 99

4.2.1. Spark Plasma Sintering 99

4.2.2. Casting 102

4.2.3. Plasma Spray 103

4.3. Microstructure Evaluation 105

4.3.1. BNNT Integration and Distribution 105

4.3.2. Interfacial Reactions 105

4.4. Multi-scale Mechanical Characterization 106

4.4.1. Nanoscale Mechanical Properties 106

4.4.2. Meso-scale Indentation Deformation 107

4.4.3. Bulk-scale Mechanical Properties 107

4.4.4. In-situ Investigation of Deformation Mechanisms 108

4.5. Remarks 111

$\begin{array}{ll}\text { References } & 112\end{array}$ 
5. PHYSICAL, CHEMICAL AND MECHANICAL CHARACTERISTICS OF BORON NITRIDE NANOTUBE AT ELEVATED TEMPERATURES 114

5.1. Thermal Transformations in Boron Nitride Nanotubes 114

5.1.1. Simultaneous Thermal Analysis $\quad 114$

5.1.2. Phase Transformations 116

5.1.3. Oxidative Functionalization 118

5.1.4. Morphological Transformations 119

5.1.5. Discussion of Oxidative Unzipping Mechanism 122

5.1.6. Discussion of White Graphene Oxide Formation Mechanism 124

5.2. High-Temperature Mechanical Properties of Boron Nitride Nanotube 126

5.2.1. Local Indentation Compression 126

5.2.2. Cyclic Indentation Response 131

5.2.3. High Temperature Tensile Deformation 137

5.2.4. Viscoelasticity at Elevated Temperatures 146

$\begin{array}{ll}\text { 5.3. Summary } & 150\end{array}$

Output: Published Articles 151

$\begin{array}{ll}\text { References } & 151\end{array}$

6. PATHWAYS FOR ENGINEERING BORON NITRIDE

NANOTUBE-ALUMINUM COMPOSITES 155

6.1. Aluminum Reinforced with Aligned BNNTs by Sintering $\begin{array}{ll}\text { and Cold Working } & 155\end{array}$

6.1.1. Interfacial Reaction Products 156

6.1.2. Tensile Properties 157

6.1.3. Fracture Surface Examination 159

6.1.4. Discussion of Strengthening Mechanisms 163

6.2. Reactive Interface Engineering in Al-BNNT by Equilibrium $\begin{array}{ll}\text { Solidification } & 167\end{array}$

6.2.1. Interactions at BNNT-Molten Al Interface 169

6.2.2. Reactive Wetting of BNNT by Molten Al 171 
6.2.3. Reactive Filling of BNNT by Molten Al 176

6.2.4. Mechanical Response 180

6.3. Layer-by-Layer Deposition of Al-BNNT by Plasma Spray 182

6.3.1. Survival and Integration of BNNT 182

6.3.2. Interfacial Interactions in Plasma 186

6.3.3. Mechanical Reinforcement 189

6.4. Summary Remarks 191

Output: Published Articles 191

References 192

7. APPLICATION OF BORON NITRIDE NANOTUBE FOR REINFORCING MAGNESIUM ALLOY 196

7.1. Sandwich Architecture to Suppress Embrittlement 196

7.2. Integration of BNNT in AZ31 197

7.3. Metal-Nanotube Interface Analysis 199

7.4. Strengthening Mechanisms 203

7.5. Summary 206

Output: Published Articles 206

References 206

8. IN-SITU INVESTIGATION OF DEFORMATION MECHANISMS INDUCED BY BORON NITRIDE NANOTUBES AND NANO-INTERPHASES IN Ti-6Al-4V 208

8.1. Background: Sintering of BNNT-reinforced Ti6Al4V Alloy 208

8.2. BNNT-induced Strengthening: Ex-situ Mechanical Investigations 210

8.3. In-situ Indentation Investigation 214

8.4. Unraveling Sub-Surface Deformation Mechanisms in Real Time 215

8.4.1. Low-Temperature Sintered Composite 220

8.4.2. High-Temperature Sintered Composite 226

8.4.3. Reinforcement Efficacy of Deformation Mechanisms 231 
8.5. Correlation between Deformation Mechanisms and Interface Chemistry 233

8.6. Summary

References

9. SUMMARY, CRITICAL APPRAISAL AND FUTURE OUTLOOK

9.1. Chemical Stability and Structural Integrity of BNNT at Elevated

Temperatures

9.1.1. Key Findings

9.1.2. Considerations

9.2. BNNT Alignment to Maximize Mechanical Strengthening of Aluminum 247

$\begin{array}{ll}\text { 9.2.1. Key Findings } & 247\end{array}$

9.2.2. Limitations and Considerations 248

9.3. Integration of BNNT in Aluminum by Equilibrium Solidification $\quad 249$

9.3.1. Key Findings 249

$\begin{array}{ll}\text { 9.3.2. Considerations } & 250\end{array}$

9.4. Layer-by-Layer Build-up of Al-BNNT by Plasma Spray 251

9.4.1. Key Findings 251

9.4.2. Concerns and Considerations 252

9.5. Integration of BNNT in a Matrix with Low Ductility

(AZ31 Magnesium Alloy) 252

9.5.1. Key Findings 252

9.5.2. Considerations 254

9.6. In-situ Study of Mechanical Interactions between Matrix, Nanotubes $\begin{array}{ll}\text { and Interphases } & 255\end{array}$

9.6.1. Key Findings 255

9.6.2. Concerns and Considerations 256

9.7. Future Directions 257

9.7.1. Interfacial Reactions vis-à-vis Mechanical Properties 257

9.7.2. Exploration of Post-Processing Design Space 258

9.7.3. Mechanical Evaluation under Diverse Stress-States 259 
9.7.4. Alternative Manufacturing Approaches

9.7.5. Multifunctional Properties

References

APPENDIX 


\section{LIST OF TABLES}

TABLE

PAGE

2.1. Small volume fabrication of BNNT-MMCs: Manufacturing processes vis-à-vis

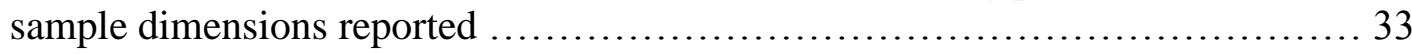

2.2. Fabrication method, morphology, dimensions, and impurities in BNNTs used for fabricating metal matrix composites reported in the literature ................... 46

2.3. Summary of mechanical reinforcement effect reported in literature, the dimensions of nanotube employed and a theoretical estimate of geometric percolation

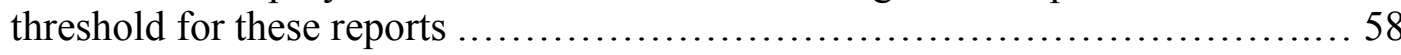

4.1. SPS processing parameters for consolidation of BNNT-MMC systems 101

6.1. Microstructure-sensitive mechanical signature from as-solidified Al-BNNT composite

8.1. Comparison of stress and energy parameters associated with deformation mechanisms observed via in-situ investigations

9.1. Summary of interfacial reaction products formed in three different metal matrices and three different processing approaches explored in this dissertation. 257 


\section{LIST OF FIGURES}

FIGURE

PAGE

1.1. Ashby-type diagram showing the disadvantage of lightweight metals for loadbearing applications (in comparison to high density, high strength steels) ..........2

1.2. Variation in relative strength (a) and relative modulus (b) of Al-CNT composites with respect to unreinforced $\mathrm{Al}$, showing strengthening and stiffening effect of

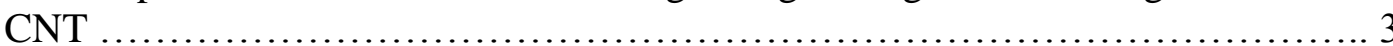

1.3. Ball-Stick models showing the side and front views of hexagonal boron nitride

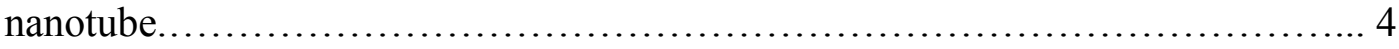

1.4. Schematic summarizing the key foci of the dissertation 11

2.1. Differential thermal analysis and thermogravimetric analysis comparing the behavior of CNT and BNNT ropes, (b) pronounced oxidation/ structural alteration of coarse BNNT after exposure to $900^{\circ} \mathrm{C}$, (c) retention of structure and morphology for finer BNNT even after exposure to $900^{\circ} \mathrm{C}$, and (d) XRD examination of phase transformations in BNNT due to thermal exposure (700 and

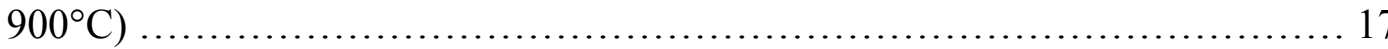

2.2. Tensile characterization of BNNT inside TEM for (a) smooth, cylindrical nanotube, and (b) bamboo-shaped nanotube with discontinuous lattice

2.3. Bending deformation mechanisms observed inside TEM for: (a) coarse BNNT,

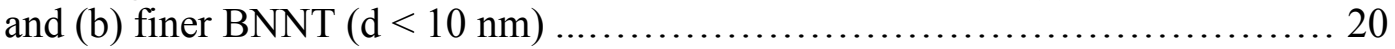

2.4. Radial compression-decompression of BNNT by an AFM tip, and (b) radial elastic modulus of BNNT before and after high temperature exposure $\left(300-850^{\circ} \mathrm{C}\right)$

2.5. Test setup for examining torsional mechanics of BNNT (suspended BNNT with a pedal on top is twisted by AFM loading), and (b) torque-torsion response over

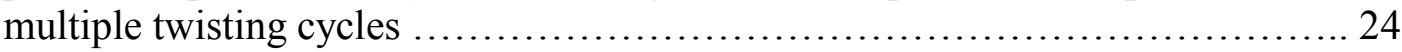

2.6. Al-BNNT composite powder prepared by wet-mixing: (a) 2 wt.\% BNNT, and (b)

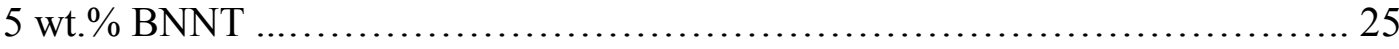

2.7. SEM micrographs showing variable Al coating thickness on BNNT (by varying

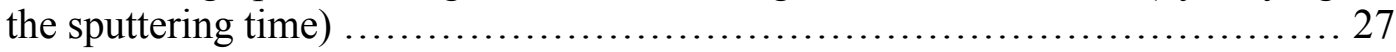

2.8. Metal ion implantation for fabricating BNNT-MMC ...................... 28

2.9. Schematic representation of the melt spinning process for fabricating Al-BNNT ribbons

2.10. Schematic representation of spark plasma sintering for consolidating Al-BNNT

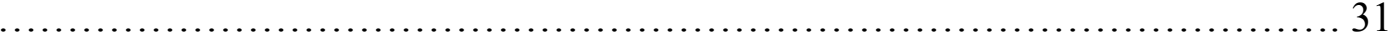

2.11. Schematic representation of high pressure-torsion consolidation of Al-BNNT .. 32

2.12. Microstructure assessment of sputter-coated Al-BNNT nanohybrids: (a) SEM image and the corresponding EDS map showing uniform coating of Al, (b) TEM 
micrographs showing uniform coating of polycrystalline $\mathrm{Al}$ on BNNT, and (c)

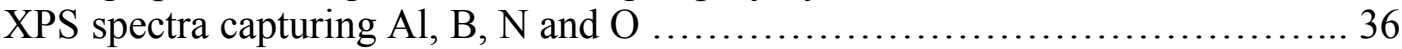

2.13. Low-to-high energy regimes for metal ion implantation to fabricate BNNT-MMC structures, and (b) Raman spectra of pristine BNNT and Al-BNNT structures indicates amorphization due to ion implantation ............................. 37

2.14. SEM micrographs showing BNNT in Al pellet (before melt spinning) and Al ribbon (after melt spinning), and (b) HRTEM images showing the integration of $\mathrm{BNNT}$ in $\mathrm{Al}$ (inside grains as well as along grain boundaries) ................. 38

2.15. Formation of $\mathrm{AlN}$ and $\mathrm{AlB}_{2}$ due to interfacial reactions between $\mathrm{Al}$ and BNNT

2.16. SEM micrographs of spark plasma sintered Al-BNNT showing: (a) signature of ductile failure, (b) pull-out of nanotube, (c) micro-porosity around grain boundaries, and (d) clustering of BNNT at grain boundaries ...

2.17. Differences in BNNT retention/ integration, and (b) EELS mapping of Al-BNNT interface in HPT-consolidated Al-BNNT composite

2.18. Comparison of FTIR spectra for BNNT before and after functionalization, and (b) SEM micrograph of the fractured surface of Al-BNNT composite fabricated using amino functionalized nanotubes

2.19. Microhardness of HPT-processed Al-BNNT: (a) Spatial variation from center to perimeter of the pellet, and (b) effect of BNNT volume fraction and heattreatment (the numeral after $\mathrm{Al}$ indicates BNNT weight percentage and the numeral after $\mathrm{BN}$ indicates heat-treatment temperature)

2.20. Micropillar compression response of SPS-fabricated Al-BNNT composite, and (b) high magnification imaging of fracture mechanisms after pillar failure ....

2.21. Effect of heat-treatment on tensile strength (a) and tensile strain (b) of HPTfabricated Al-BNNT composites

2.22. In-situ TEM tensile deformation of sputter-coated Al-BNNT nanohybrid and the corresponding force-displacement response

2.23. Pull-out of inner BNNT shells during tensile loading of Al-BNNT, and (b) high magnification image of the Al-BNNT interface from where BN shells are pulled

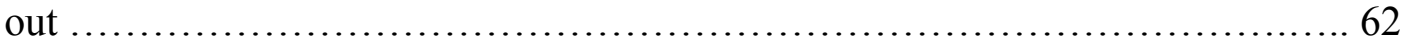

2.24. Real-time TEM snapshots of sputter-coated Al-BNNT nanocomposite undergoing bending deformation

3.1. The relative position of a ( $20 \%$ dense) BNNT agglomerate in the Ashby map, showing reasonably strong inter-tube bonding in the clusters ..................... 73

3.2. A schematic representation highlighting the importance of nanotube orientation in MMC microstructure for load-bearing ability owing to BNNT's high aspect ratio 
3.3. (a) Kinetics of Al-BNNT interfacial reactions (reaction products as a function of thermal exposure time), and (b) tensile strength and yield strength of Al-CNT as a function of interfacial reaction product fraction ....

3.4. (a) Illustration of an energy barrier for nanomaterial capture in molten metal due to repulsive forces, and (b) SEM micrograph of a melt-spun Al-BNNT composite with poor integration highlighting the importance of meeting capture criterion ....82

3.5. (a) Effect of temperature and (b) effect of nanofiller volume fraction on the

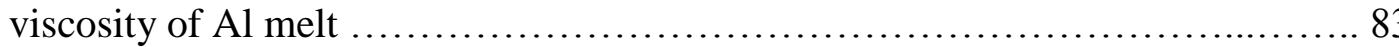

3.6. (a) TEM micrograph of a CNT-Al composite exhibiting dislocation loop formation, and (b) computed dislocation density as a function of nanotube content demonstrating dislocation pileups in the microstructure because of 1D

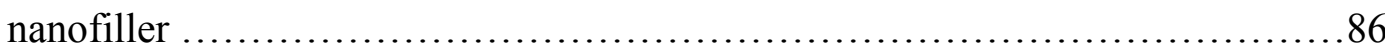

3.7. Comparison of micromechanics models and experimental data points for CNTMMC, highlighting the difficulty of predicting the elastic modulus

3.8. An illustration of multi-scale deformation phenomena in nanotube-reinforced MMCs

4.1. FESEM image of the as-received dense cluster of nanotubes (inset shows the as received ball of entangled BNNTs), and (b) FTIR spectrum of as-received BNNTs

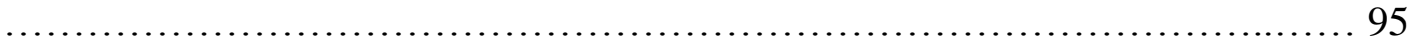

4.2. Sonication and surfactant-assisted BNNT de-agglomeration and dispersion in

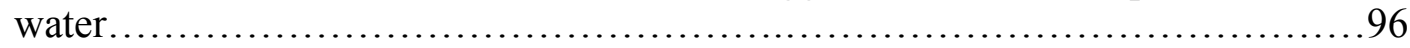

4.3. SEM micrographs of BNNT buckypaper microstructure: (a) a network of high aspect ratio nanotubes randomly aligned to form mesh-like architecture (inset shows a free-standing, foldable buckypaper), (b) high magnification micrograph of a network of nodes/ intertube junctions and nanotube struts, and (c) crosssection of the buckypaper comprising of multiple layers of BNNT meshes with inter-layer nanotube linkages providing structural support.................... 99

4.4. FESEM images of as-received (a) $\mathrm{Al}$, and (b) AZ31 powders for fabricating BNNT-MMCs

4.5. Schematic representation of the process of sputter coating, sandwiching and aligning long BNNTs within the Aluminum matrix, and the actual pictures of the composite sample at each stage. Similar sintering approach was adopted for AZ31-BNNT, except the final rolling step.................................102

4.6. A solidified and ground Al-BNNT composite sample fabricated by casting .....103

4.7. Dispersed Al-BNNT feedstock powder for plasma spray deposition obtained by

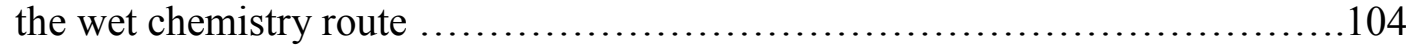

4.8. Focused ion beam milling of Ti-BNNT composite to expose the internal structure for observing the deformation mechanisms 
4.9. In-situ double cantilever test set-up for examining strengthening mechanisms at $\mathrm{Mg}-\mathrm{BNNT}$ interface in a sandwich-structured composite ....................110

5.1. (a) Weight change of BNNT, and (b) heat required to increase the temperature of the BNNT sample during TGA-DSC, as a function of temperature............... 115

5.2. Post thermal analysis phase characterization of BNNT for different TGA-DSC maximum temperatures by X-Ray Diffraction ........................... 117

5.3. Fourier Transform Infrared Spectrum (FTIR) of BNNT sample subjected to TGA$\mathrm{DSC}$ for varying temperatures, ranging from room temperature to $1000^{\circ} \mathrm{C}$

5.4. SEM images of BNNT sample at different TGA-DSC maximum temperatures showing: (a) pure nanotube cluster at room temperature, (b) growth of nodules in nanotube network at $500^{\circ} \mathrm{C}$, (c) formation of thin stacks of 2-D plates while retaining nanotube clusters at $750^{\circ} \mathrm{C}$, and (d) the formation of dense and thick 2-D stacks of white graphene oxide, with further reduction in the number of nanotube clusters. (e) EDS spectrum of platelets revealed $\mathrm{O}$..................121

5.5. (a) High magnification image showing the co-existing nanotube and platelet network, and (b) Schematic diagram showing two-stage BNNT to WGO transformation at elevated temperatures. Activation barrier for oxidative unwrapping of BNNTs via Stone Wales defect site and pure site is compared ... 123

5.6. SEM images showing progression of oxidative transformation of BNNT: (a) unreacted, partially uncurled and completely transformed regions marked yellow, (b) sideways growth of 2D WGO platelet by unzipping and coalescence of BNNTs, (c) uncurled nanoribbon forming a new peel over previous layer in WGO stack, and(d) fully formed thick stack of WGO layers, with some dangling nanotubes visible along the sideways

5.7. (a) In-situ nanoindentation of BNNT buckypaper inside SEM chamber: indentation compression leads to elastic stretching of buckypaper due to highly interconnected network, (b) nanoindentation load-displacement response of buckypaper at room temperature, $250^{\circ} \mathrm{C}$ and $500^{\circ} \mathrm{C}$ temperatures, (c) local compressibility and elastic modulus of buckypaper determined during nanoindentation as a function of temperature, and (d) schematic representation of BNNT buckling/ kinking mechanism active during indentation compression 128

5.8. (a) Nanoindentation load-displacement response for cyclic loading-unloading (50 cycles) with hysteresis loops at room temperature, $250^{\circ} \mathrm{C}$ and $500^{\circ} \mathrm{C}$, (b) microstructure strain contours developed in the buckypaper during indentation loading for 1 stand 50 thcycles are shown, attesting long-distance stress-transfer and accumulation of residual strain over the 50 deformation cycles (scale bar 20 $\mu \mathrm{m}$ ), and (c) schematic representation of the detachment-attachment mechanism at nanotube junctions leading to topological transformation due to stretching of buckypaper (during cyclic indentation)

5.9. Tensile stress-strain curves for BNNT buckypaper deformed at room temperature, $250^{\circ} \mathrm{C}, 500^{\circ} \mathrm{C}$ and $750^{\circ} \mathrm{C}$ 
5.10. Sequence of deformation events recorded by high speed camera at 5,000 frames per second during tensile testing of BNNT buckypaper at room temperature ....139

5.11. Scanning electron micrographs, revealing tensile failure mechanisms at different temperatures: (a) delamination of layers in the assembly, (b) morphological transformations at elevated temperatures, (c) alignment of nanotube bundles due to tensile loading, and (d) comparison of inter-layer load-bearing mechanism at room temperature and $750^{\circ} \mathrm{C}$

5.12. (a) Thermo-mechanical deformation of BNNT buckypaper exposed to a contact heating element during high temperature tensile testing, (b) infrared thermal image/map of the buckypaper experiencing tensile loading, and (c) the corresponding temperature profile along the buckypaper length

5.13. (a) Loss tangent as a function of dynamic loading frequency for BNNT buckypaper recorded at room temperature, $250^{\circ} \mathrm{C}, 500^{\circ} \mathrm{C}$ and $750^{\circ} \mathrm{C}$, and (b) the schematic illustration of the unzipping-zipping mechanism responsible for viscoelasticity in BNNT buckypaper assembly

6.1. X-ray diffraction pattern for spark plasma sintered layered Al-BNNT-Al

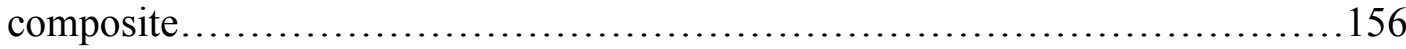

6.2. Tensile stress-strain behavior for layered Al-BNNT-Al composite showing multi-

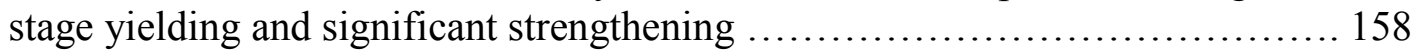

6.3. SEM image showing composite fracture surface, with BNNTs pulled out along the tensile axis. Inset shows the typical ductile mode of failure shown by Aluminum

6.4. SEM images showing: (a) crack-bridging due to directionally aligned BNNTs, and (b) enlarged view of a BNNT network acting as crack bridges along the entire depth of the crack section.

6.5. SEM image showing detachment of interfacial product and pulled out nanotubes along $\mathrm{Al} / \mathrm{BNNT}$ interface after tensile failure

6.6. Schematic diagram summarizing strengthening and deformation mechanisms in Al-BNNT-Al sandwich composite

6.7. (a) SEM image showing a network of long nanotube clusters well meshed in $\mathrm{Al}$ matrix post solidification, (b) low magnification TEM image showing long and intact BNNTs in cast Al-BNNT composite, and (c) phases identified in solidified Al-BNNT composite ................................................... 167

6.8. High resolution TEM and lattice images showing: (a) formation of AlN at AlBNNT interface, and (b) complete coverage of nanotube walls by AlN

6.9. (a) Schematic representation of AIN induced wetting of BNNT by molten Al, and

(b) theoretical calculations for the work of adhesion as a function of the extent of

AlN formation at Al-BNNT interface ..................................... 172 
6.10. SEM image showing BNNT completely wrapped by molten Al, confirming excellent interfacial wetting. Inset shows a high magnification image of nanotubes uniformly coated by Al.

6.11. TEM images showing filling of BNNTs: (a) moderate capillarity represented by nano-droplets, and (b) strong capillary effect resulting in filling by larger rod-

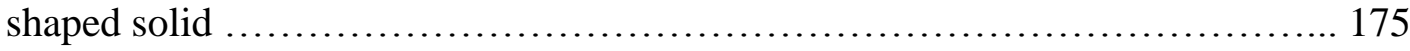

6.12. Variation of Laplace pressure with contact angle and the schematic representation of capillarity-induced filling ................................. 178

6.13. Analysis of reaction between BNNT and $\mathrm{Al}$ inside the nanotube 180

6.14. Microstructure-sensitive local response captured by nanoindentation of the assolidified Al-BNNT composite

6.15. (a) SEM micrograph of the polished cross-section of plasma sprayed coating, showing swirly splats as the fundamental microstructural building blocks, (b) SEM micrograph showing long nanotubes in the microstructure, (c) high magnification SEM micrograph showing excellent wetting of BNNT during plasma spray, and (d) X-ray diffraction characterization of plasma-sprayed deposit confirms retention of $\mathrm{h}-\mathrm{BN}$ phase in $\mathrm{Al}$, along with minimal interfacial reactions

6.16. (a) Low magnification TEM micrograph showing integration of long nanotube in the matrix, (b) high magnification TEM micrograph showing a network of nanotubes in the matrix, and (c) STEM micrograph of the nanotube in the matrix and the corresponding EDX spectrum line profile confirming the survival of BN nanotube during plasma spray

6.17. (a) HRTEM image and analysis showing reaction products at $\mathrm{Al} / \mathrm{BNNT}$ interface, and (b) SAED pattern obtained from a nanotube that confirms the formation of AIN during plasma spray....

6.18. Nanoindentation load-displacement curves exhibiting the non-homogeneous mechanical response of the coating. Enhanced indentation-resistance in certain regions of the coating indicates BNNT reinforcement

7.1. Processing scheme of AZ31-BNNT sandwich-structured composite by highpressure spark plasma sintering

7.2. (a) X-ray diffraction plot showing $\mathrm{h}-\mathrm{BN}, \mathrm{Mg}$, and $\mathrm{MgO}$ in the sintered pellet, (b) low magnification SEM micrograph revealing multiple crack deflection events (inset: image of the sectioned and delaminated pellet used for imaging), (c) SEM micrograph showing a stepped surface and multi-layer cracking due to sectioning, and (d) high magnification SEM micrograph demonstrating impregnation and intimate bonding of alloy with the nanotubes....

7.3. (a) STEM imaging and the corresponding elemental line profile along the alloyBNNT interface region, and (b) HRTEM image showing a dense network of BNNTs in the composite 
7.4. Lattice spacing calculations confirming: (a) h-BN phase of the nanotubes, (b) $\mathrm{Mg}_{3} \mathrm{~N}$ 2formation, and (c) AlN formation due to AZ31-BNNT reaction ........ 202

7.5. In-situ double cantilever testing of AZ31-BNNT composite with real-time SEM imaging: (a) stretching and fracture of BNNTs resisting crack opening, and (b) snapping of composite cluster acting as interface anchor. Post-delamination imaging showing (c) extensive crack bridging and (d) nanotube pull-out due to mechanical loading....................................................205

8.1. Comparison of meso-scale indentation curves for Ti6Al4V alloy and BNNTreinforced Ti6Al4V composites (LT and HT-sintering conditions) ....

8.2. Post indentation FESEM imaging of deformation in (a) LT-sintered, and (b) HT-sintered Ti6Al4V-BNNT composites

8.3. In-situ indentation for observing deformation mechanisms in Ti6Al4V alloy (a), LT Alloy-BNNT composite (b), and HT Alloy-BNNT composite (c) ......... 214

8.4. FIB milling of Ti6Al4V-BNNT composites for real time imaging of internal structure during indentation loading

8.5. (a) Programmed loading cycles for in-situ testing of milled trenches in displacement-control mode, and (b) illustration of work expended and recovered in an indentation cycle

8.6. Force-displacement response and in-situ SEM images demonstrating crack initiation, propagation and widening along the alloy particle boundaries in LT composite

8.7. (a) SEM micrograph showing particle detachment due to mechanical loading, and (b) imaging at multiple indentation depths reveal transition from intrinsic plasticity to inter-particle de-bonding deformation in LT-Ti6Al-BNNT.

8.8. (a) Real-time SEM images reveal the plastic flow of material without cracks or particle de-bonding, and (b) SEM images of a separate region where excess clusters resulted in poor adhesion and catastrophic failure....................224

8.9. In-situ indentation showing onset of shear banding (a), and log-range shear band propagation with subsequent loading cycles (b)

8.10. Force-displacement response and in-situ SEM images showing time-resolved crack initiation and propagation in HT-sintered Ti6Al4V-BNNT.

8.11. In-situ SEM images showing transition from tortuous crack propagation (a), to local plasticity (a), to pronounced plastic flow of material (c) .................230

8.12. SEM imaging after indentation tests reveals crack bridging and pull-out of long nanotubes in LT-sintered composite (a) and needle-like nano-species in HTsintered composite (b).

8.13. HRTEM investigation of Ti6Al4V-BNNT composites showing retention of long nanotubes in LT-sintered composite (a), co-existence of BNNT with TiN and TiN 
interphases in LT-sintered composite (b), and significant reactions/ phase transformations in HT-sintered composite (c)

8.14. (a) XPS B1s and N1s spectra for LT and HT composites, and (b) micro-scale chemical mapping of the composite microstructure with superimposed images comparing the relative atomic fraction of $\mathrm{BN}$ and interphases $\ldots \ldots \ldots \ldots \ldots \ldots . \ldots 238$

9.1. Schematic summarizing the focus areas of this dissertation and key conclusions

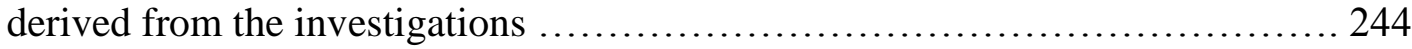




\section{ABBREVIATIONS AND ACRONYMS}

\begin{tabular}{|c|c|}
\hline BNNT & Boron Nitride Nanotube \\
\hline DIC & Digital Image Correlation \\
\hline EDS & Electron Dispersive Spectroscope/ Spectroscopy \\
\hline FIB & Focused Ion Beam Machining \\
\hline FTIR & Fourier Transform Infrared Spectroscope/ Spectroscopy \\
\hline HPT & High Pressure Torsion \\
\hline HRTEM & High Resolution Transmission Electron Microscope/ Microscopy \\
\hline SEM & Scanning Electron Microscope/ Microscopy \\
\hline SPS & Spark Plasma Sintering \\
\hline STEM & Scanning Transmission Electron Microscope/ Microscopy \\
\hline MMC & Metal Matrix Composite \\
\hline TEM & Transmission Electron Microscope/ Microscopy \\
\hline UTS & Ultimate Tensile Strength \\
\hline XPS & X-ray Photoelectron Spectroscope/ Spectroscopy \\
\hline XRD & X-ray Diffraction \\
\hline
\end{tabular}




\section{CHAPTER 1 INTRODUCTION}

The objective of this study is to engineer metal-matrix composites with exceptional mechanical properties by utilizing hexagonal boron nitride nanotube as the reinforcement phase and investigate the fundamental deformation mechanisms at multiple microstructural length scales. The motivation behind this research is to augment the load-bearing ability of existing lightweight metals and alloys (with high strength-to-weight ratio), such as aluminum, magnesium and titanium, so that they can be employed as critical structural members for aerospace, automotive and marine applications.

\subsection{Lightweight Metal Matrix Composites}

There has been a great interest in engineering lightweight structural materials for highspeed and long-distance transportation because of advantages in energy efficiency, reduced fuel consumption, controlled carbon footprint and better acceleration. As a result, metals with high strength-to-weight ratio, such as titanium, aluminum and magnesium are desirable for aerospace, marine and automotive applications. However, the mechanical properties of these metal systems fall short for critical structural applications where high strength steels are used. Figure 1.1 shows an Ashby type diagram for yield stress vs. ductility highlights the disadvantage of $\mathrm{Al}, \mathrm{Mg}$ and $\mathrm{Ti}$ alloys when compared with high strength steels. ${ }^{1}$ It is vital to fill this gap in mechanical performance to broaden the applicability of lightweight metals. 


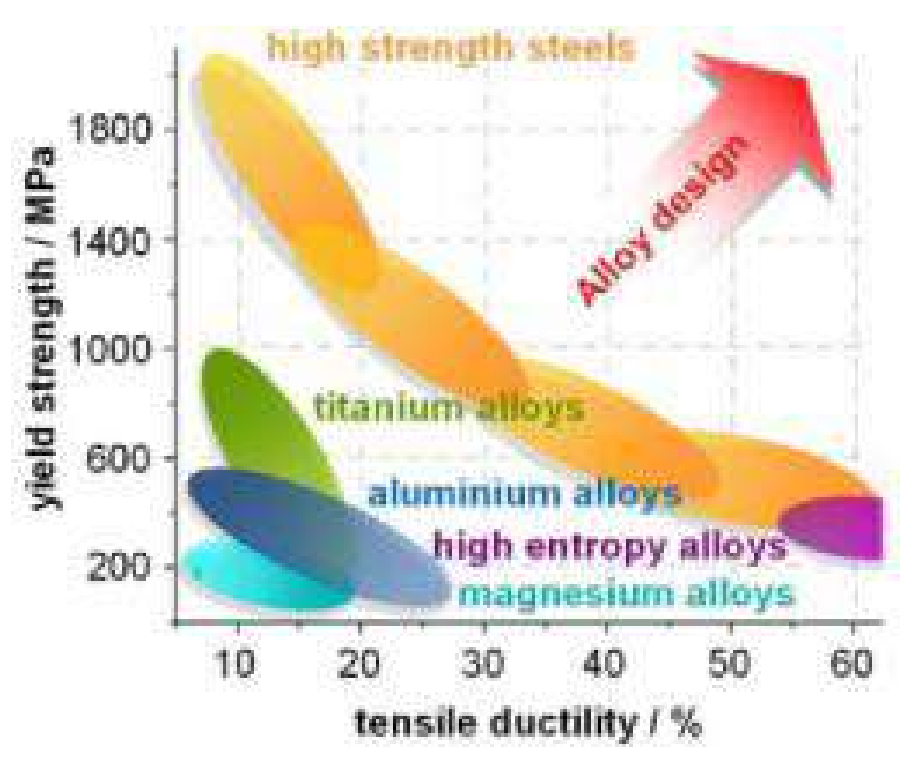

Figure 1.1. Ashby-type diagram showing the disadvantage of lightweight metals for loadbearing applications (in comparison to high density, high strength steels). ${ }^{1}$

Reinforcing these low-density metals with secondary fillers helps in augmenting their mechanical properties, such as stiffness, strength, hardness, toughness, fatigue resistance, wear resistance etc. One-dimensional (1D) fillers, with a fibrous or tubular architecture, are particularly desirable because of their rope-like crack-bridging potential, high surface area for interface bonding and their potential to form interconnected network in the composite microstructure for mechanical/ geometric percolation. Use of highstrength carbon fibers gained tremendous popularity starting in 1960s for manufacturing advanced composite materials, with applications in rocket nozzles, missile nose tips, heat shields, aircraft brakes, wings and fuselage, and thermal management. In 1991, the discovery of carbon nanotubes (CNT) revolutionized the field of composite materials. CNTs, with diameter about 3 orders of magnitude smaller than the carbon fibers display exceptional mechanical properties, such as stiffness up to $\sim 1 \mathrm{TPa}$ and tensile strength 
exceeding $11 \mathrm{GPa}$. This inspired the use of CNT as a reinforcement for metal matrices, such as $\mathrm{Al}, \mathrm{Cu}, \mathrm{Ni}, \mathrm{Mg}$, Ti etc. ${ }^{2}$ The addition of $\mathrm{CNT}$ to $\mathrm{Al}$ matrices has been reported to result in up to three-fold enhancement in fracture strength (Figure 1.2a) and up to 75\% improvement in elastic modulus (Figure 1.2b). ${ }^{3}$
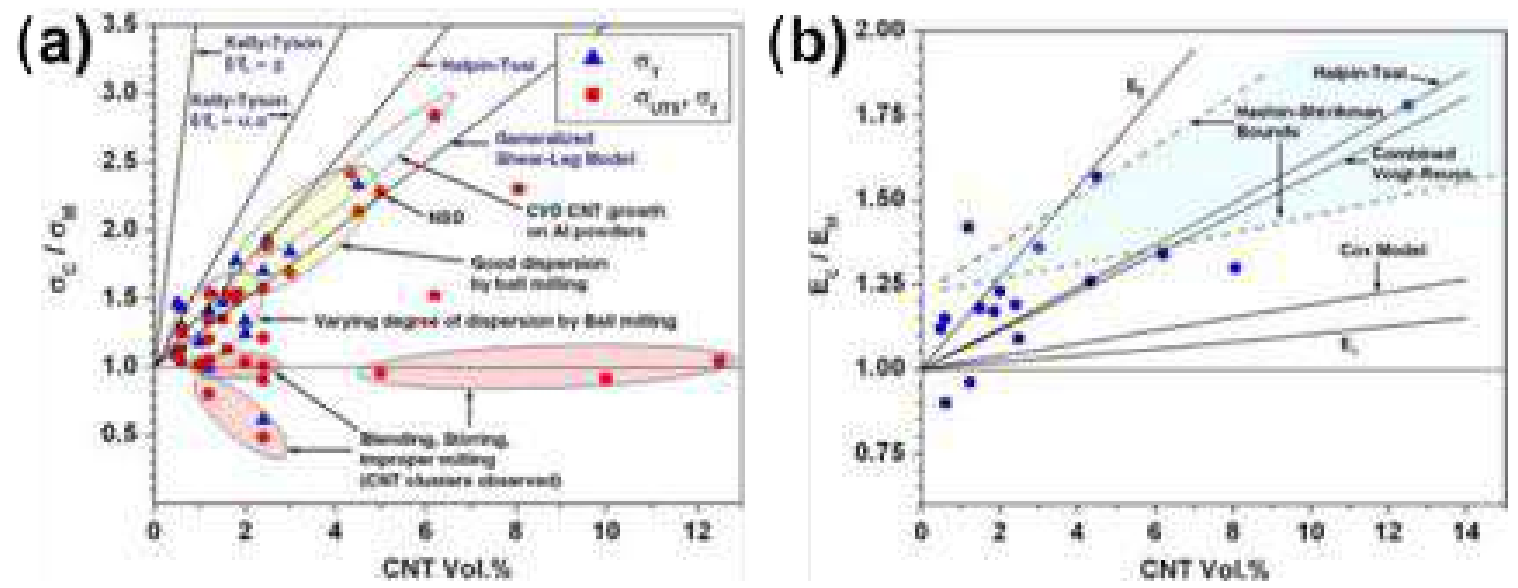

Figure 1.2. Variation in relative strength (a) and relative modulus (b) of Al-CNT composites with respect to unreinforced $\mathrm{Al}$, showing strengthening and stiffening effect of $\mathrm{CNT}^{3}$

One major drawback of CNT for engineering metal matrix composites is its poor structural and chemical stability at elevated temperatures. CNT oxidizes when exposed to temperatures exceeding $400^{\circ} \mathrm{C}$, which limits the processing window for manufacturing metal matrix composites. Metalworking operations involve high temperature exposure, which is conducive to chemical reactions between the nanotubes and the metal matrices. Excess reactions during processing compromises the structural integrity and mechanical properties of the nanotubes. As a result, high temperature exposure needs to be limited for manufacturing CNT-based composites. Moreover, CNT-based composites cannot be employed for high temperature structural applications due to this reason. Therefore, despite 
their attractive mechanical properties, CNT is not a very effective secondary filler for metal matrix composites.

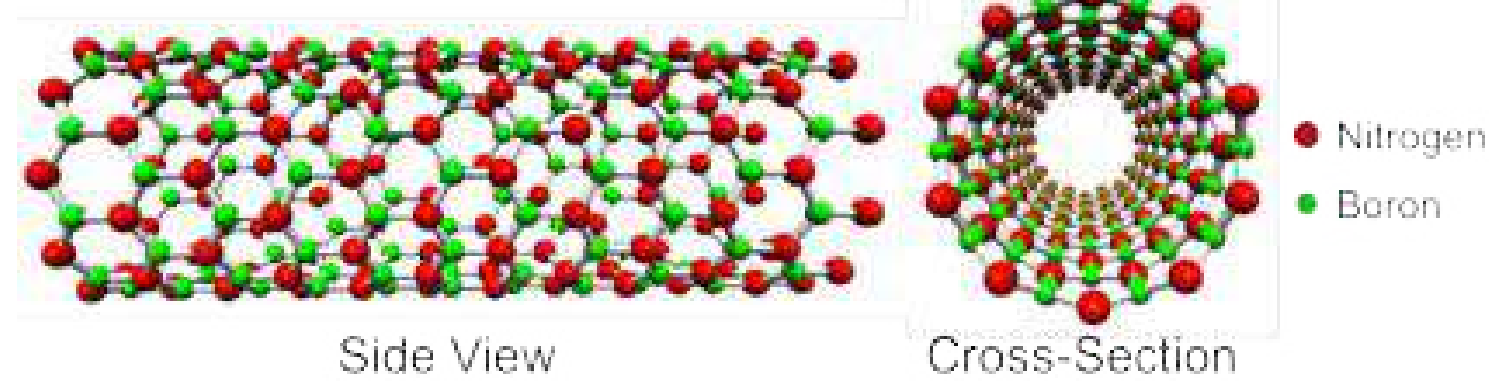

Figure 1.3. Ball-Stick models showing the side and front views of hexagonal boron nitride nanotube.

\subsection{Boron Nitride Nanotube as a Structural Reinforcement}

BNNT, a 1D nanomaterial and a structural analogue of CNT, consists of alternating B and $\mathrm{N}$ atoms arranged in a honeycomb-like fashion (Figure 1.3). Like CNT, BNNT also displays excellent intrinsic mechanical properties, such as elastic modulus and tensile strength exceeding $1 \mathrm{TPa}$ and $60 \mathrm{GPa}$, respectively. ${ }^{4}$ Additionally, BNNT has two major advantages over CNT: (i) BNNT has superior high temperature endurance, as it can resist oxidative transformations up to $\sim 700-900^{\circ} \mathrm{C}^{5,6}$ and (ii) BNNT is chemically more stable (less reactive) in comparison to $\mathrm{CNT},{ }^{5,7}$ This makes it a compatible nanofiller with metal manufacturing processes. Moreover, BNNT has several other desirable characteristics, such as excellent thermal conductivity (as high as $2,400 \mathrm{~W} \mathrm{~m}^{-1} \mathrm{~K}^{-1}$ for aligned nanotubes), ${ }^{8}$ neutron radiation absorption ability, ${ }^{9}$ and it is a piezoelectric material. ${ }^{10,11}$ These characteristics are promising in aerospace applications - structural, packaging as well as devices - where thermal management, radiation shielding, and energy harvesting are desired. The fact that BNNT can display brilliant functional properties, in addition to 
remarkable thermal and mechanical resilience, makes it a highly suitable nanofiller for engineering multifunctional structural composites.

\subsection{Challenges and Limitations in Current Understanding}

\subsubsection{Nanotube Morphology and Purity}

Strengthening in a composite is dictated by the stress-transfer from the matrix to the filler phase. For 1D nanotube reinforced composites, longer tubes are desirable for effective strengthening and crack-bridging mechanisms. The early studies on BNNT-MMCs relied on shorter nanotubes $(<10 \mu \mathrm{m})$ fabricated by mechanothermal, annealing and chemical vapor deposition techniques. ${ }^{12,13}$ Low aspect ratio nanotubes are characterized by relatively lower surface area, lowering the total matrix/filler interfacial area available for stresstransfer to take place. Fabrication by ball milling introduces elemental impurities, such as $\mathrm{Fe}$ and $\mathrm{Cr}$ to BNNT. These elements can react and form undesirable phases during MMC processing. Some of the studies also employ bamboo-shaped BNNTs, which do not have smooth morphology and are characterized by discontinuous lattices separated by joints. As a result, bamboo-shaped nanotubes display relatively inferior mechanical properties, with the experimentally measured fracture strength and Young's modulus $\sim 8 \mathrm{GPa}$ and $225 \mathrm{GPa}$, respectively. ${ }^{14}$ These values are roughly a quarter of the mechanical properties displayed by smooth, crystalline BNNTs. Use of short, impure and segmented nanotubes fails to realize the reinforcement potential of BNNTs. 


\subsubsection{Metal Matrix Composite Processing}

Scalable manufacturing is vital for commercial application of novel high-performance composites. The early works on BNNT-MMCs made use of processing techniques, such as magnetron sputtering, ${ }^{15}$ high-pressure torsion (HPT), ${ }^{16}$ pressureless vacuum sintering,,${ }^{17}$ spark plasma sintering (SPS) ${ }^{18}$ and melt spinning. ${ }^{19}$ Most of these techniques can produce very small volume of materials, with centimeter-scale or smaller dimensions. Techniques such as SPS or HPT require somewhat complex equipment setup, highly skilled labor and these are not as widely used in the manufacturing industry. It is desirable to explore the development of BNNT-MMCs by other manufacturing approaches, especially those which are easily scalable and commercially viable. For instance, casting (metal solidification) is one of the most extensive and traditional manufacturing techniques. There is a lack of information whether BNNT can survive the extreme thermal conditions in a molten metal pool, the nature of chemical interactions between molten metal and nanotubes, and the microstructure characteristics in cast BNNT-MMCs. In last one decade, interest in additive manufacturing (AM) has grown exponentially because layer-by-layer material build-up enables the fabrication of net-shaped or near net-shaped structures. However, AM techniques are characterized by non-equilibrium conditions due to ultrafast cooling rates. The possibility, challenges and strategies for BNNT integration via AM processes are currently unknown. The current gap in fundamental understanding of composite processing via scalable manufacturing processes is a major roadblock in the widespread adoption of BNNTs as structural reinforcement filler for metal matrices. 


\subsubsection{Challenges with Mechanical Properties}

Some early studies have reported the advantageous effect of BNNT addition in Aluminum, such as improvement in hardness, ${ }^{17}$ compressive yield strength, ${ }^{18}$ elastic modulus ${ }^{19}$ and tensile strength. ${ }^{16,19}$ However, the degree of improvement is highly variable in these studies and the enhancement in properties is often nominal, not meeting up to the reinforcement potential of BNNT. There are several factors which are responsible for this observation:

- Effective enhancement in properties requires that the added nanofiller should exceed the mechanical percolation threshold, which is a theoretical volume fraction at which a connected microstructure is formed. ${ }^{20}$ This threshold is a function of nanotube dimensions, and exceeding the threshold usually results in a prominent improvement. The studies on BNNT-MMCs have not examined the mechanical percolation aspect.

- Increasing the BNNT volume fraction in the composite should resolve the abovementioned percolation limitation. However, nanotubes have very high surface area and van der Waals forces cause them to agglomerate. Due to their tubular architecture, BNNT are also seen to entangle with each other, forming dense clusters. It is challenging to ensure homogeneous distribution of nanotubes throughout the metal matrix. BNNT clusters act as stress concentration sites in the microstructure and can lead to failure initiation. In fact, one study on Al-BNNT reports drop in hardness with BNNT addition due to the accumulation of the nanotubes. ${ }^{16}$ Dispersion becomes even more critical for scalability, as poor nanotube distribution in a large-scale sample will result in inconsistent and inferior mechanical performance. There has been a lack of 
attention on strategies for BNNT dispersion to design homogeneous MMC microstructures.

- 1D nanomaterials display superior mechanical properties along the axial length, and generally possess rather low radial strength and stiffness. Therefore, only the nanotubes aligned along the loading direction (in a uniaxial test) are expected to contribute towards the load-bearing capacity. Even if the theoretical percolation threshold is reached, the mechanical anisotropy of BNNT implies not all the fibers will equally contribute towards strengthening. The studies on BNNT-MMCs have largely ignored the orientation-dependence during composite engineering.

- Stress-transfer between matrix and filler is the fundamental requirement for nanofiller strengthening. This requires intimate interface adhesion. Weak interfaces can easily fail under mechanical loading. Currently, there is no study correlating the mechanical properties with the nature of interface bonding between BNNT and metal matrices. The lack of effort towards characterizing the nature of interfaces is a contributing factor towards the observed anomalies in the mechanical performance of BNNT-MMCs.

- Introduction of a secondary filler can significantly alter the deformation characteristics of metals: they arrest plasticity, cause deflection and bridging of cracks, undergo interface sliding, and experience stress-induced pull-out and fracture. Investigation of deformation mechanisms in BNNT-MMCs is restricted to post-mortem imaging of the samples, after the mechanical testing is over. Considering the complex microstructure of the composites, post-failure imaging fails to provide a complete picture of the efficacy of BNNTs for mechanical reinforcement. 


\subsection{Objectives of the Research}

Based on the discussion in the preceding sections, it is hypothesized that BNNT's mechanical properties can be efficiently harnessed and translated to large-scale metal matrix composites by careful control over nanotube alignment, nanotube dispersion and interface chemistry. This necessitates the investigation of microstructure evolution vis-àvis processing approach and conditions. Further, it is vital to understand deformation events that are activated in the MMCs to design composite microstructures for desired mechanical characteristics. This work delves into these fundamental scientific and materials engineering aspects pertaining to BNNT-MMCs. The three key elements of this dissertation are:

(1) Investigation of thermal and mechanical characteristics of BNNT to evaluate its suitability as a mechanical reinforcement for metal matrices:

- Physical and chemical transformations experienced at elevated temperatures

- Assessment of load-bearing ability at elevated temperatures

(2) Exploration of multiple, scalable manufacturing strategies for effectively engineering composite microstructures:

- Nanotube dispersion and alignment in metal matrices

- Identification of suitable processing parameters

- Metal-BNNT chemical reactions during processing

- Integration of BNNT in different metal matrices: (a) low melting point aluminum- and magnesium-based composites, and (b) high melting point titanium-based composites 
(3) Examination of mechanical properties of the synthesized nanocomposites at multiple length scales:

- Composites subjected to different stress-states

- Assessing improvement in mechanical properties

- Real-time imaging of deformation mechanisms

The research objectives and approaches undertaken in this dissertation are summarized in Figure 1.4. 
Thermal Characteristics of BNNT Physical-Chemical Transtomations High-Temperature Mechanics
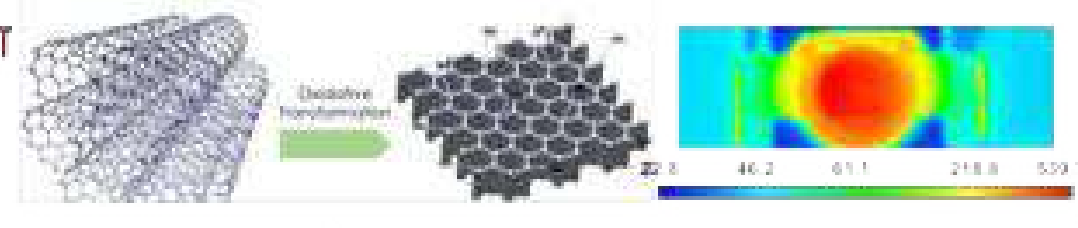

Microstructure Engineering

Processing Route:

Dispersion and Algnment

Intertace and Reactions

integration in Metal Matrices
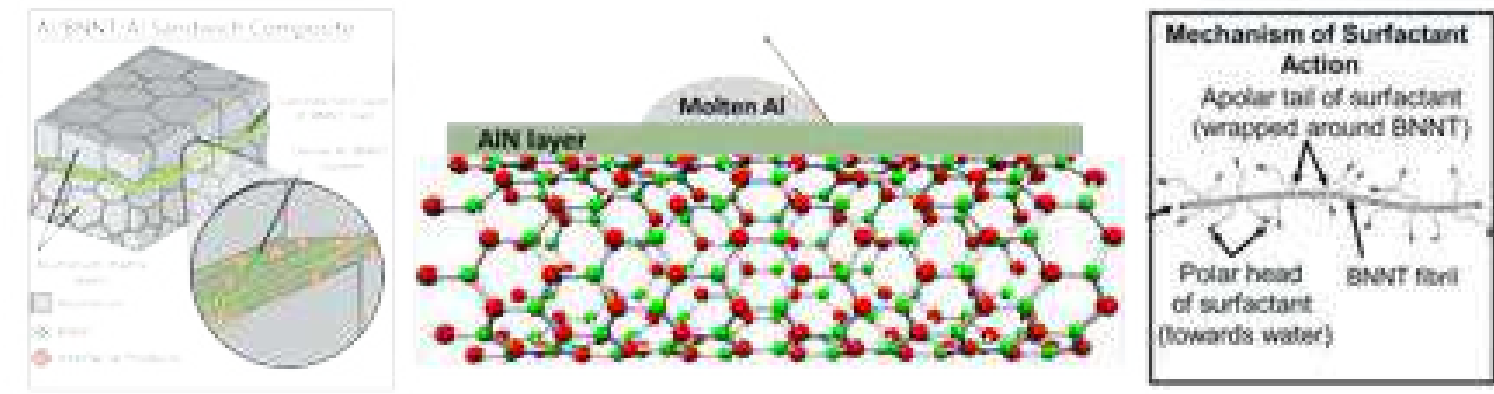

\begin{abstract}
Multi-Scale Mechanics Nechanical Improvement Strengthening Wechomism Real-time Imaging
\end{abstract}

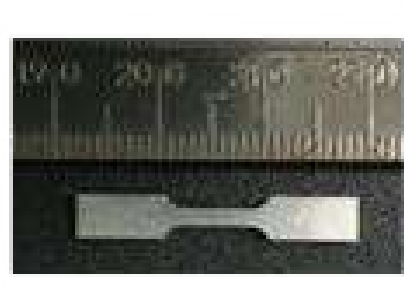

$m m-m$

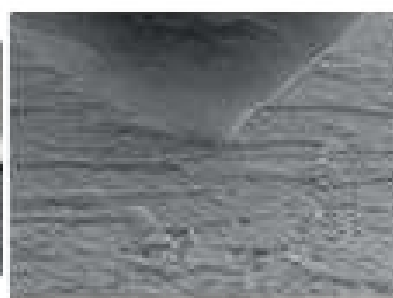

$\mu m-m m$

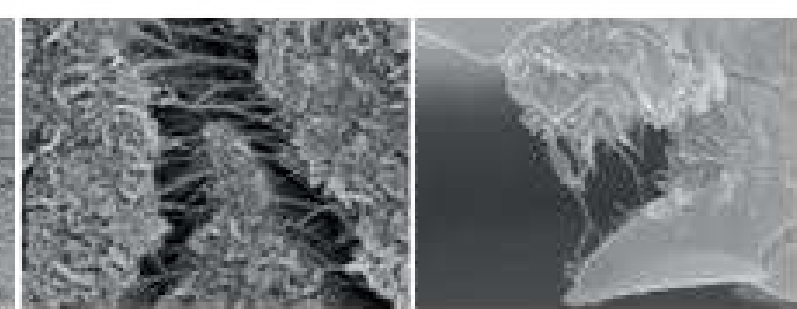

$\mu m-n m$

Figure 1.4. Schematic summarizing the key foci of the dissertation. 
This dissertation is divided into 9 chapters. Chapter 2 provides a critical review of the state-of-the-art in BNNT technology, such as physical and chemical characteristics of BNNT, mechanical and functional properties of BNNT, and processing and mechanical behavior of BNNT-MMCs. Chapter 3 is focused on the theoretical framework and fundamental concepts, which have inspired the approaches and directions pursued in the dissertation. The materials, experimental methods, tools and techniques used to meet the dissertation objectives are introduced in Chapter 4. Chapter 5 describes the investigation of physical, chemical and mechanical characteristics of BNNT at elevated temperatures. Chapters 5 through 7 are the heart of the dissertation, where manufacturing, microstructure evolution, interface chemistry and mechanical properties of BNNT-MMCs are scrutinized. Chapter 6 delves into pathways for engineering BNNT-reinforced aluminum composites with desired microstructure characteristics and superior mechanical properties. Chapter 7 demonstrates the application of BNNT for reinforcing a lightweight magnesium alloy by employing ingenious physical-chemical interface engineering strategies. The real-time imaging of deformation mechanisms by high-resolution in-situ mechanical investigations is presented in Chapter 8. A titanium alloy-based composite system is chosen for in-situ assessment because it's a high-temperature material, providing a wider processing window for interrogating the effect of interfacial reactions on the mechanics of MMC. The dissertation ends with a chapter on critical appraisal of the findings of the thesis and a discussion on the future outlook for BNNT-MMCs. 


\section{References}

1. Springer, H., Baron, C., Szczepaniak, A., Uhlenwinkel, V. \& Raabe, D. Stiff, light, strong and ductile: nano-structured High Modulus Steel. Sci. Rep. 7, 2757 (2017).

2. Agarwal, A., Bakshi, S. R. \& Lahiri, D. Carbon Nanotubes: Reinforced Metal Matrix Composites. (CRC Press, Taylor \& Francis Group, 2011).

3. Bakshi, S. R. \& Agarwal, A. An analysis of the factors affecting strengthening in carbon nanotube reinforced aluminum composites. Carbon N. Y. 49, 533-544 (2011).

4. Wei, X., Wang, M.-S., Bando, Y. \& Golberg, D. Tensile tests on individual multiwalled boron nitride nanotubes. Adv. Mater. 22, 4895-4899 (2010).

5. Golberg, D., Bando, Y., Kurashima, K. \& Sato, T. Synthesis and characterization of ropes made of BN multiwalled nanotubes. Scr. Mater. 44, 1561-1565 (2001).

6. Chen, Y., Zou, J., Campbell, S. J. \& Caer, G. Le. Boron nitride nanotubes: Pronounced resistance to oxidation. Appl. Phys. Lett. 84, 2430-2432 (2004).

7. Lahiri, D. et al. Insight into reactions and interface between boron nitride nanotube and aluminum. J. Mater. Res. 27, 2760-2770 (2012).

8. Belkerk, B. E. et al. Thermal conductivity of vertically aligned boron nitride nanotubes. Appl. Phys. Express 9, 075002 (2016).

9. Thibeault, S. A. et al. Nanomaterials for radiation shielding. MRS Bull. 40, 836$841(2015)$

10. Bai, X. et al. Deformation-driven electrical transport of individual boron nitride nanotubes. Nano Lett. 7, 632-637 (2007).

11. Kang, J. H. et al. Multifunctional Electroactive Nanocomposites Based on Piezoelectric Boron Nitride Nanotubes. ACS Nano 9, 11942-11950 (2015).

12. Tang, C., Bando, Y., Sato, T. \& Kurashima, K. A novel precursor for synthesis of pure boron nitride nanotubes. Chem. Commun. 1290-1291 (2002).

13. Li, L. H., Chen, Y. \& Glushenkov, A. M. Synthesis of boron nitride nanotubes by boron ink annealing. Nanotechnology 21, 105601 (2010).

14. Tang, D.-M. et al. Mechanical properties of bamboo-like boron nitride nanotubes by in situ TEM and MD simulations: Strengthening effect of interlocked joint interfaces. ACS Nano 5, 7362-7368 (2011). 
15. Yamaguchi, M., Tang, D., Zhi, C., Bando, Y. \& Shtansky, D. Synthesis, structural analysis and in situ transmission electron microscopy mechanical tests on individual aluminum matrix / boron nitride nanotube nanohybrids. Acta Mater. 60, 6213-6222 (2012).

16. Yamaguchi, M., Meng, F., Firestein, K., Tsuchiya, K. \& Golberg, D. Powder metallurgy routes toward aluminum boron nitride nanotube composites, their morphologies, structures and mechanical properties. Mater. Sci. Eng. A 604, 9-17 (2014).

17. Singhal, S. K., Srivastava, A. K., Pasricha, R. \& Mathur, R. B. Fabrication of AlMatrix Composites Reinforced with Amino Functionalized Boron Nitride Nanotubes. J. Nanosci. Nanotechnol. 11, 5179-5186 (2011).

18. Lahiri, D. et al. Boron nitride nanotubes reinforced aluminum composites prepared by spark plasma sintering: Microstructure, mechanical properties and deformation behavior. Mater. Sci. Eng. A 574, 149-156 (2013).

19. Yamaguchi, M. et al. Utilization of multiwalled boron nitride nanotubes for the reinforcement of lightweight aluminum ribbons. Nanoscale Res. Lett. 8, 3 (2013).

20. Wang, G., Yu, D., Mohan, R. V., Gbewonyo, S. \& Zhang, L. A comparative study of nanoscale glass filler reinforced epoxy composites: Electrospun nanofiber vs nanoparticle. Compos. Sci. Technol. 129, 19-29 (2016). 


\section{CHAPTER 2}

\section{A CRITICAL REVIEW OF THE STATE-OF-THE-ART}

This chapter provides relevant background information about boron nitride nanotube (BNNT) and an overview of the current state-of-the-art related to BNNT-based metal matrix composites (BNNT-MMCs). The chapter starts with a discussion on the thermal stability and oxidation mechanisms in BNNT, followed by an overview of key mechanical characteristics of BNNT reported in the literature. Thereafter, a critical assessment of processing, microstructure study and mechanical investigations of BNNT-MMCs is presented. The chapter ends with a discussion on the current gap in scientific understanding in the form of some critical questions that need to be answered for meaningful advancements in the field of BNNT-MMCs. The goal of this chapter is to overhaul the scientific literature, critically examine the lacunae in the reported approaches, and build a case for the investigations reported in the subsequent chapters.

\subsection{Thermal Stability of Boron Nitride Nanotubes}

One of the striking characteristics of BNNT is its resistance to oxidation and chemical stability at elevated temperatures. There are reports establishing that BNNT's oxidation resistance extends up to twice the temperatures that CNT is able to endure ${ }^{1,2}$. Golberg et al. reported differential thermal analysis (DTA) and thermogravimetry (TGA) of CNT and BNNT ropes made of chemically vapor deposited nanotubes ${ }^{1}$. While CNT started oxidizing around $571^{\circ} \mathrm{C}$, the reaction initiation in the case of BNNT was observed only after $830^{\circ} \mathrm{C}$ 
(Figure 2.1a). In a separate study, Chen and co-workers performed TEM examination of BNNTs after exposing them to $700-900^{\circ} \mathrm{C}$ temperatures ${ }^{2}$. The study reported a thin and disordered layer of oxide formed on BNNT after heating up to $700^{\circ} \mathrm{C}$. Further heating to $900^{\circ} \mathrm{C}$ produced interesting observations: while coarse nanotubes $(20-200 \mathrm{~nm}$ diameter) were severely damaged, the finer BNNTs (diameter $<20 \mathrm{~nm}$ ) did not degrade after hightemperature exposure (Figure 2.1b). TGA revealed a sharp weight gain around $800^{\circ} \mathrm{C}$, which the authors ascribed to BNNT oxidation. In contrast to BNNT, CNT oxidation initiated around $400^{\circ} \mathrm{C}$ and was completed at $700^{\circ} \mathrm{C}$. XRD examination of BNNT before and after thermal exposure revealed extensive oxidation at $900^{\circ} \mathrm{C}$ : the diffraction intensity of $\mathrm{BN}$ peaks was significantly suppressed and the peak intensity of $\mathrm{B}_{2} \mathrm{O}_{3}$ was pronounced (Figure 2.1c).

TEM imaging indicated that oxidation initiates at BNNT tips and along the broken walls. Defects in coarse-grained nanotubes, such as bamboo and cone-like structures, are detrimental to the oxidation resistance. The formation of $\mathrm{Fe}_{2} \mathrm{O}_{3}$ was also seen in the XRD spectra, and it was attributed to the presence of iron particles in the BNNT sample. The impurities were introduced during BNNT synthesis (from Fe catalyst). These findings underscore the importance of structure, morphology and purity for nanotube design and applications. Overall, the superior thermal stability of BNNT is promising for engineering metal matrix composites, which often require elevated processing temperatures. This also opens the possibility of exploring high-temperature applications of BNNT-MMCs. 

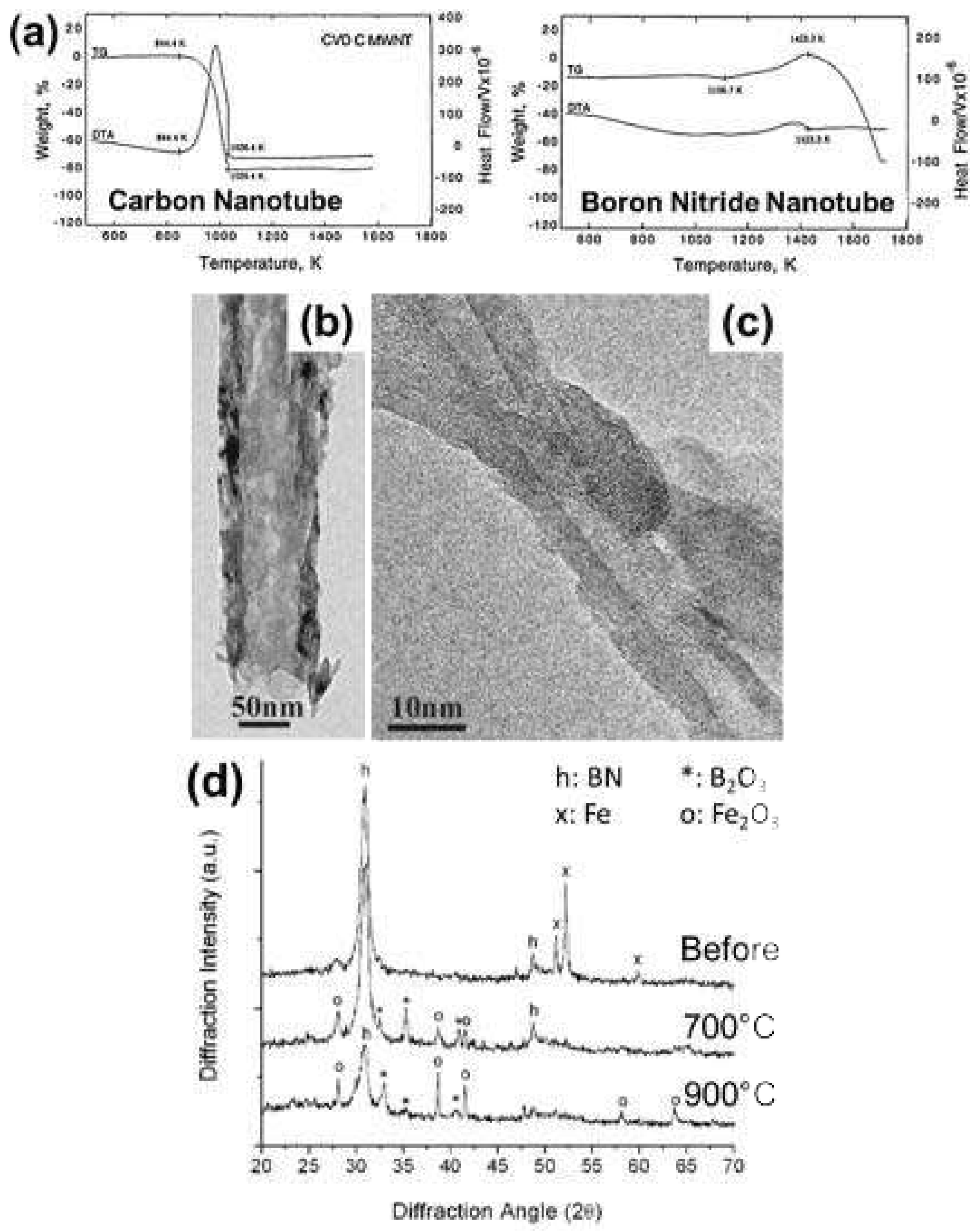

Figure 2.1. (a) Differential thermal analysis and thermogravimetric analysis comparing the behavior of CNT and BNNT ropes ${ }^{1}$, (b) pronounced oxidation/ structural alteration of coarse BNNT after exposure to $900^{\circ} \mathrm{C}^{2}$, (c) retention of structure and morphology for finer BNNT even after exposure to $900^{\circ} \mathrm{C}^{2}$, and (d) XRD examination of phase transformations in BNNT due to thermal exposure $\left(700 \text { and } 900^{\circ} \mathrm{C}\right)^{2}$. 


\subsection{Mechanical Properties of Boron Nitride Nanotubes}

\subsubsection{Tensile Behavior}

BNNT is characterized by remarkable tensile strength. In-situ tensile tests inside HRTEM have shown the measurements are sensitive to the location of failure ${ }^{3}$. The intrinsic fracture strength of BNNT varied from 26.9 to $33.2 \mathrm{GPa}$. The strength was much lower $(8.5-20.6$ GPa) when the failure happened at the clamping site because of stress concentration. Additionally, prominent structural defects such as irregular cross-section, vacancies, impurities or dislocations can also lower the strength of the nanotubes. The calculated Young's modulus ranged from 0.7 to $1.3 \mathrm{TPa}$. Pull-out of multiwalled BNNT was characterized by breakage of shells and stick-slip motion. This observation is notably different from CNTs, which generally don't exhibit breakage of shells during telescopic sliding ${ }^{4}$. These differences are ascribed to the partially ionic character of B-N bonds. This requirement of inter-shell breakage prior to failure is promising for enhanced strengthening and energy dissipation, highlighting the promise of MW-BNNT as a structural reinforcement. It has been observed that the morphology of BNNT influences its mechanical properties (Figure 2.2). Tang and co-workers examined the tensile response of a bamboo-shaped BNNT and reported much lower strength $\sim 8 \mathrm{GPa}$ and elastic modulus $\sim 0.2 \mathrm{TPa}^{5}$. The inferior mechanical properties are attributed to the discontinuous lattices separated by bamboo joints. These joints are held together by mechanical interlocking and weak van der Waals interactions. These findings highlight the importance of nanotube morphology for effective mechanical reinforcement. 


\section{(a) Uniform Cylindrical}

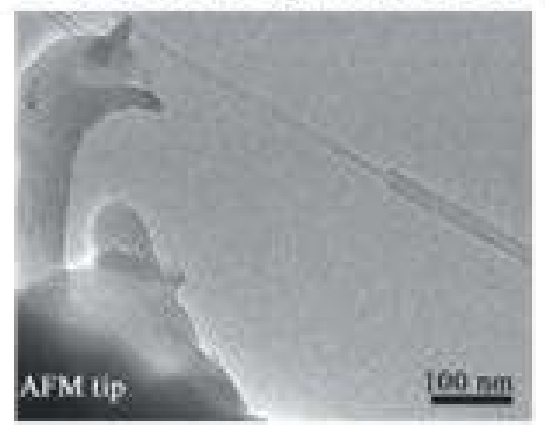

(b) Bamboo-Shaped
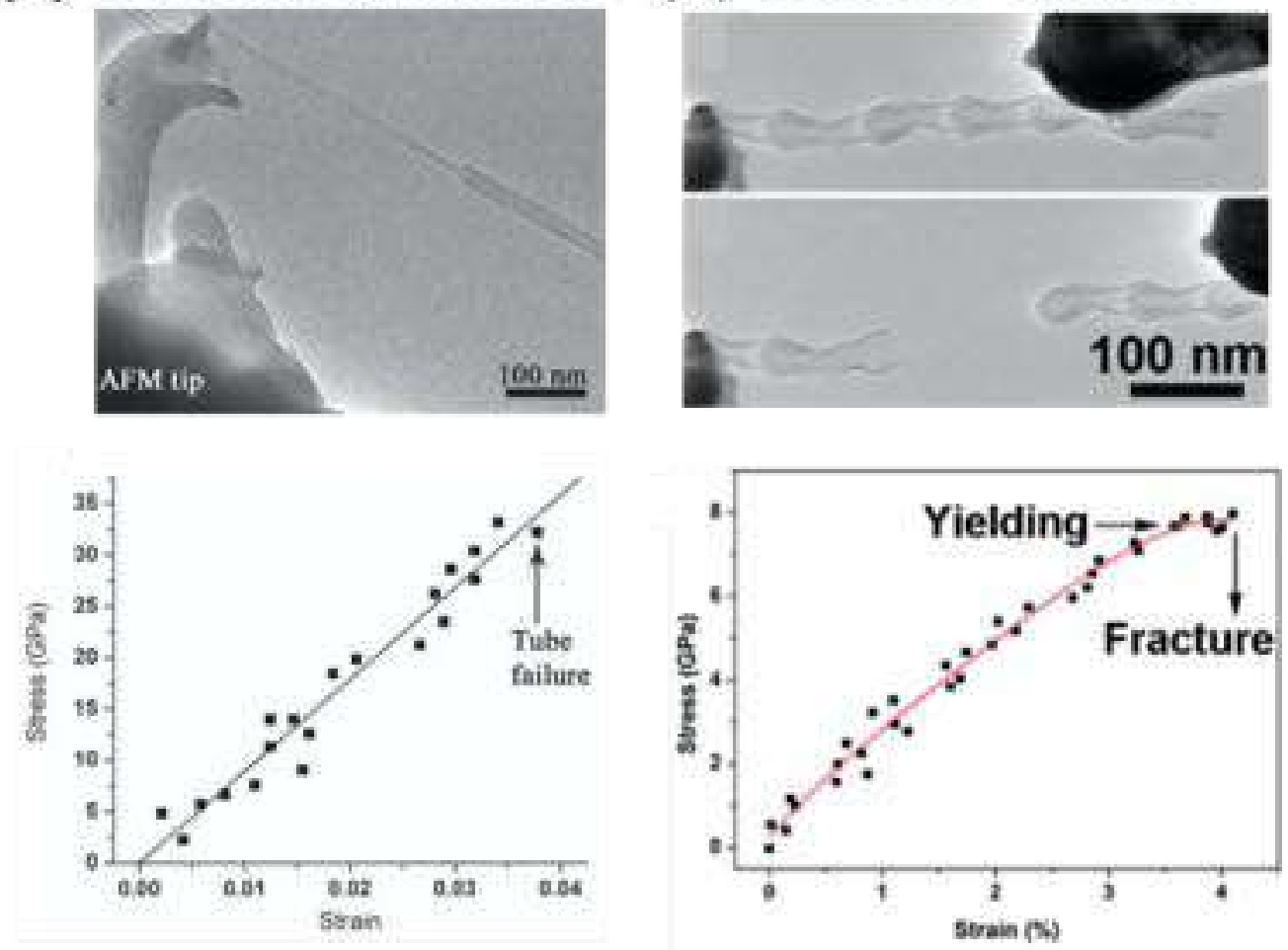

Figure 2.2. Tensile characterization of BNNT inside TEM for (a) smooth, cylindrical nanotube ${ }^{3}$, and (b) bamboo-shaped nanotube with discontinuous lattice ${ }^{5}$.

\subsubsection{Bending Behavior}

Unlike bulk ceramic materials, BNNT displays impressive flexibility. Huang et al. performed bending of BNNT inside $\mathrm{TEM}^{6}$. One end of the nanotube was mounted on a substrate and the other end contacted an AFM tip. Bending was performed by progressively reducing the substrate-AFM separation. The study reported bucking or kinking of nanotubes due to bending. The sharp kinks were associated with dislocation-like defects (Figure 2.3a). The observed bending mechanisms were influenced by the diameter of BNNT. Nanotubes with a diameter of less than $10 \mathrm{~nm}$ did not display sharp kinks; rather, ripple-like morphology was reported for finer nanotubes (Figure 2.3b). This makes finer 


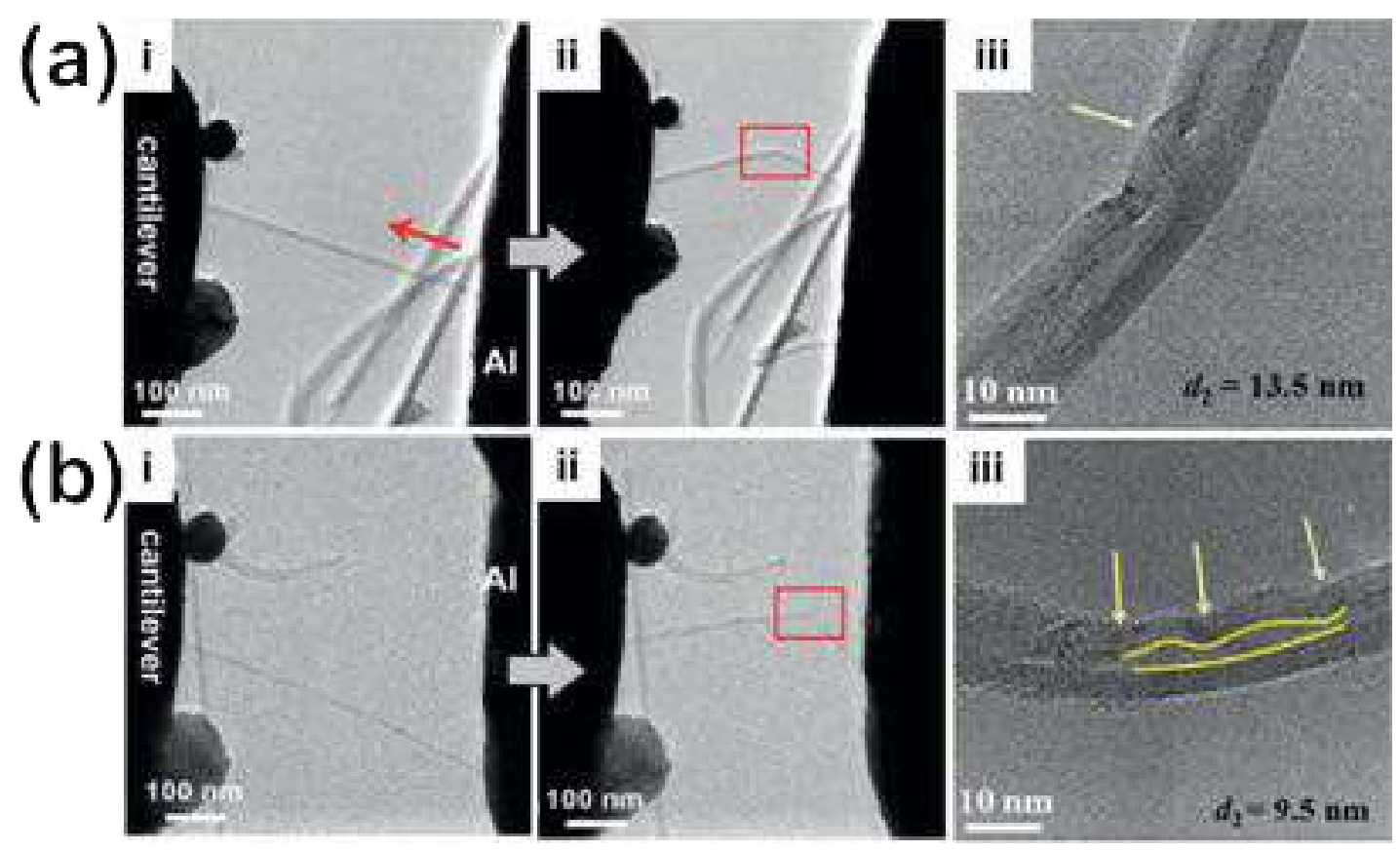

Figure 2.3. Bending deformation mechanisms observed inside TEM for: (a) coarse BNNT, and (b) finer BNNT $(\mathrm{d}<10 \mathrm{~nm})^{6}$.

BNNTs highly flexible (bending radius $\sim 45 \mathrm{~nm}$ ) and more resistant to bond breakage. The critical load for nanotube failure by buckling was estimated using Euler's formula:

$$
F_{c r}=\frac{\pi^{2} E I}{L^{2}}
$$

where $\mathrm{E}$ is Young's modulus, $\mathrm{L}$ is the nanotube length and $\mathrm{I}$ is the moment of inertia for the hollow tube, which can be determined by the relationship:

$$
I=\frac{\pi\left(d_{2}^{4}-d_{1}^{4}\right)}{64}
$$

where $d_{1}$ and $d_{2}$ are the inner and outer tube diameters, respectively. The critical force for buckling was much lower in the case of finer nanotubes $(\sim 12 \mathrm{nN})$ as compared to coarser nanotubes $(\sim 46 \mathrm{nN})$; however, finer nanotubes were able to sustain much higher forces 
even after buckling. As a result, fine BNNT (with $5 \mathrm{~nm}$ diameter) displayed an exceptionally high bending strength of $\sim 1.2 \mathrm{GPa}$. On the other hand, coarse nanotubes were characterized by a rather low bending strength of $\sim 0.3 \mathrm{GPa}$. These findings highlight the importance of nanotube dimensions for mechanical reinforcement.

\subsubsection{Radial Mechanical Properties}

BNNT displays anisotropy in mechanical properties owing to its morphology. The superior strength and stiffness of nanotubes along the axial orientation was discussed in Section 2.2.1. Zheng and co-workers measured the radial mechanical properties of BNNT deposited on Si wafer by AFM loading-unloading tests ${ }^{7}$. While lower compression loads $(5 \mathrm{nN})$ showed elastic deformation with no permanent height reduction; forces greater than

$10 \mathrm{nN}$ induced plastic deformation in the nanotubes (Figure 2.4a). The radial elastic moduli were calculated based on a Hertzian $\operatorname{model}^{8}$ :

$$
h=h_{0}-\left(\frac{P}{k_{1} \sqrt{h_{0}}}\right)^{\frac{2}{3}}-\left(\frac{P}{k_{2} \sqrt{R_{*}}}\right)^{\frac{2}{3}}+\left(\frac{P}{k_{3} \sqrt{R_{\text {tip }}}}\right)^{\frac{2}{3}}
$$

$$
k_{1}=\frac{4}{3}\left(\frac{1-v_{n t}^{2}}{E_{n t}^{r a d}}+\frac{1-v_{\text {sub }}^{2}}{E_{\text {sub }}}\right)^{-1}, k_{2}=\frac{1-v_{t i p}^{2}}{E_{\text {tip }}}, k_{3}=\frac{4}{3}\left(\frac{1-v_{t i p}^{2}}{E_{t i p}}+\frac{1-v_{\text {sub }}^{2}}{E_{\text {sub }}}\right)^{-1}
$$

where $\mathrm{P}$ is compressive load, $\mathrm{h}$ is the measured height of nanotube (on the substrate), $\mathrm{E}$ is the elastic modulus and $v$ is the Poisson's ratio. The subscripts nt, tip and sub denote BNNT, AFM tip and substrate, respectively. The authors reported diameter-dependent radial moduli for BNNT, ranging from $1.85 \mathrm{GPa}$ to $40.78 \mathrm{GPa}$. These radial modulus 
values are $20-40 \%$ lower than the moduli reported for CNT of comparable diameters. The authors remarked the strong but supple characteristic of BNNT will be useful for engineering nanocomposites, as they will be able to conform to the metal or ceramic grains for superior bonding.

(a)

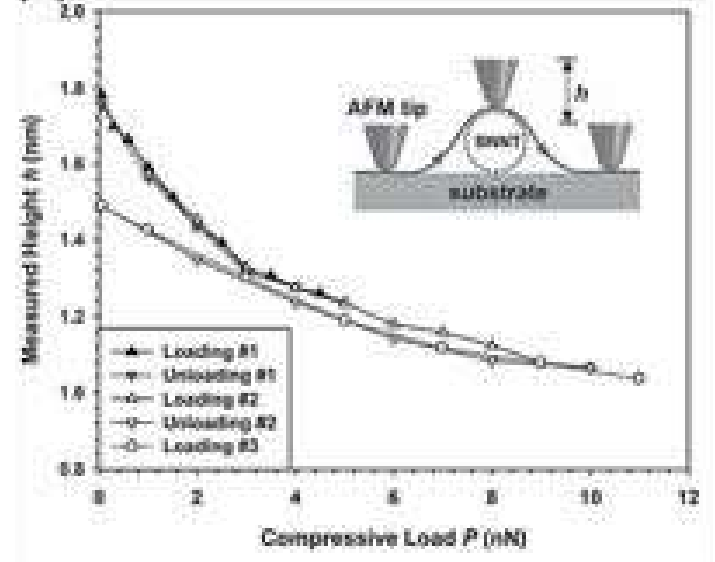

(b)

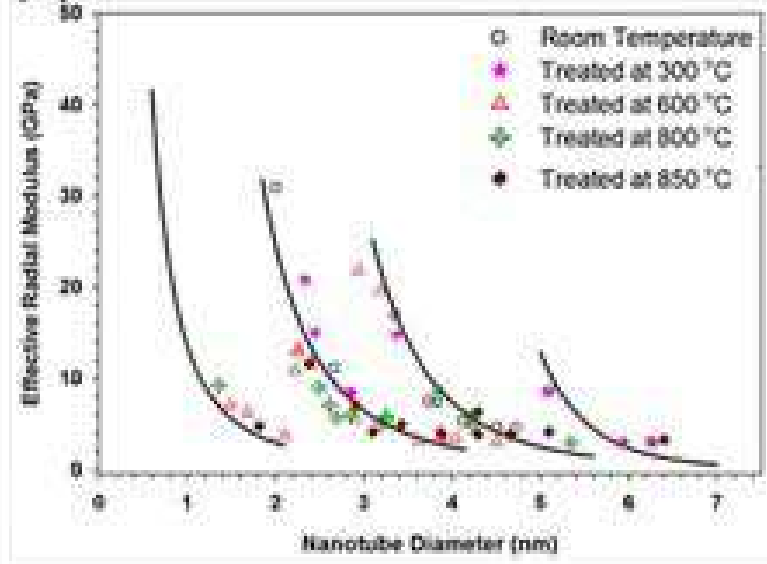

Figure 2.4. (a) Radial compression-decompression of BNNT by an AFM tip ${ }^{7}$, and (b) radial elastic modulus of BNNT before and after high temperature exposure $\left(300-850^{\circ} \mathrm{C}\right)^{9}$.

In a separate study, Chen et al. used the same approach to investigate the effect of thermal exposure on the radial mechanical response of BNNTs ${ }^{9}$. The AFM measurements were performed on the cooled down nanotubes at room temperature (Figure 2.4b). The authors reported that there was no change in the effective radial modulus of BNNTs before or after heat-treatment (up to $850^{\circ} \mathrm{C}$ ). It is noteworthy that the measurements were not performed at elevated temperatures; nevertheless, these post-cooling tests highlight that BNNT retains its mechanical integrity after thermal exposure. This finding is useful because metal/ ceramic matrix composite manufacturing typically involves elevated temperature conditions, and it is critical that the properties of BNNT should not be 
compromised during processing. However, this finding does not conclusively establish the suitability of BNNT for high temperature structural applications. There are no reports on mechanical testing of BNNTs performed at elevated temperatures, which will be necessary to understand their load-bearing ability under extreme thermal conditions.

\subsubsection{Torsional Mechanical Properties}

Mechanical interactions between nanotube walls in BNNT is an important attribute for its reinforcing potential in composite materials. Garel et al. evaluated the torsional mechanics of BNNT to interrogate the interlayer coupling in multiwalled nanotubes ${ }^{10}$. The test set-up used in the study comprised of BNNT suspended between two metallic pads and a pedal on top of the nanotube (Figure 2.5a). The pedal was pressed using an AFM tip, which caused the twisting of the nanotube. The torque-torsion relationship was seen to be reversible over multiple twisting cycles (Figure $\mathbf{2 . 5 b}$ ), indicating elastic deformation. Since BNNT did not break even after repeated twisting (up to $60^{\circ}$ angle), it was not possible to determine the failure strength. However, the authors estimated the lower bound of the torsional strength using the maximum value of torque applied during the tests:

$$
\tau_{B N N T}=\frac{T_{\max }}{\pi r_{\text {out }}^{3}}
$$

where $T_{\max }$ is the maximum torque exerted on the nanotube and $r_{\text {out }}$ is the outer nanotube diameter. Based on this relationship, the lower bound of $\tau$ was calculated to be 2 GPa. This exceeds the torsional strength of CNT $\left(\tau_{\mathrm{CNT}}=0.14-0.19 \mathrm{GPa}\right)$ by an order of magnitude! The inter-layer coupling in BNNT allows effective distribution of load, resulting in brilliant 
failure-resistance. This is an extremely important finding, and underscores the superiority of MW-BNNT over MW-CNT for engineering nanocomposites.
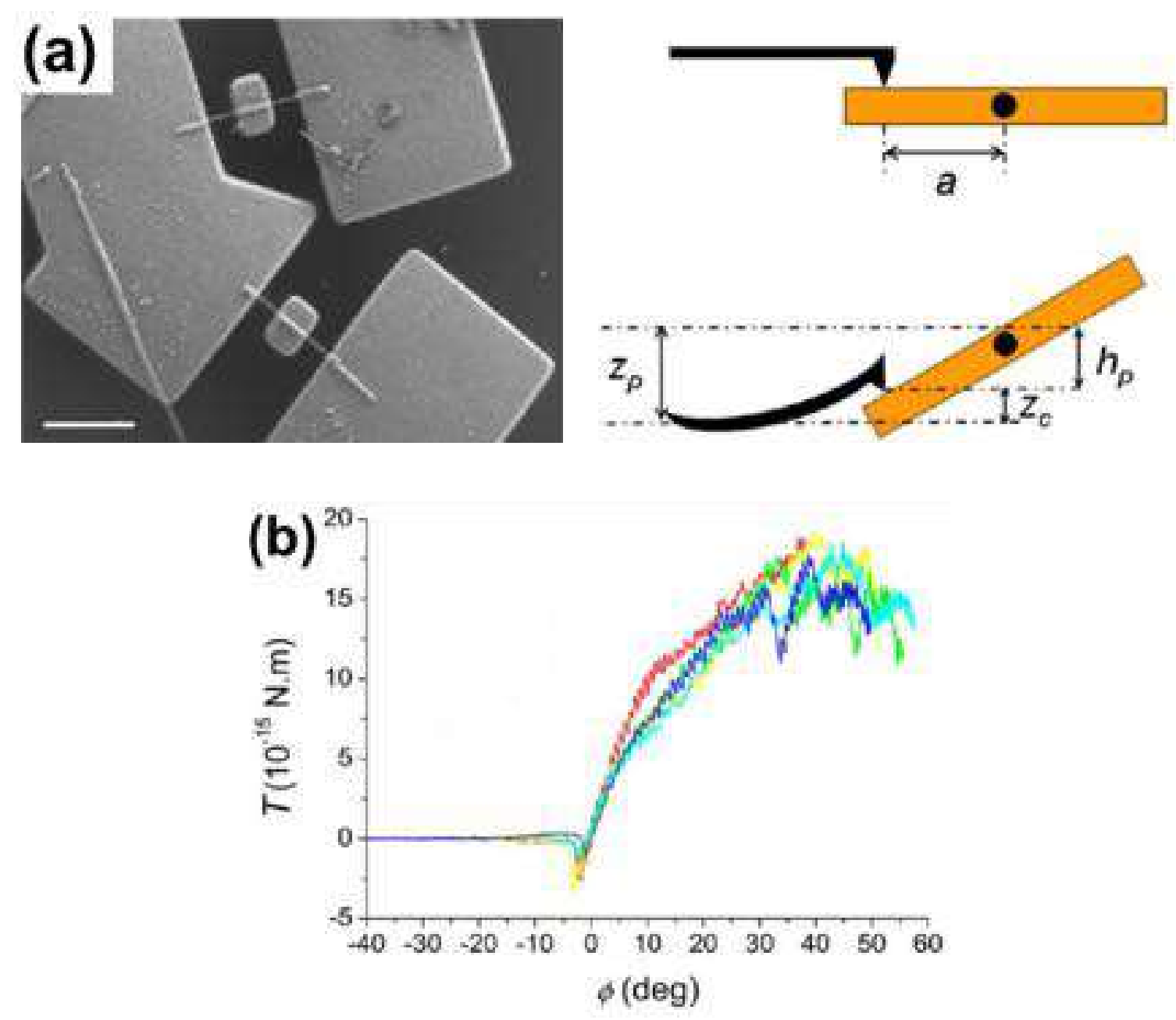

Figure 2.5. (a) Test setup for examining torsional mechanics of BNNT (suspended BNNT with a pedal on top is twisted by AFM loading), and (b) torque-torsion response over multiple twisting cycles ${ }^{10}$.

Inspired by these remarkable mechanical properties and thermal stability of BNNT, there is a rising interest in utilizing these nanotubes for reinforcing lightweight metals. The subsequent sections of the chapter are devoted to BNNT-MMCs. 


\subsection{Processing of Boron Nitride Nanotube-Metal Matrix Composites}

\subsubsection{Synthesis of Composite Precursor}

Mixing of BNNT and metal matrix is a critical step prior to composite manufacturing. The microstructure homogeneity and mechanical properties of the MMC depend on the composite precursor.
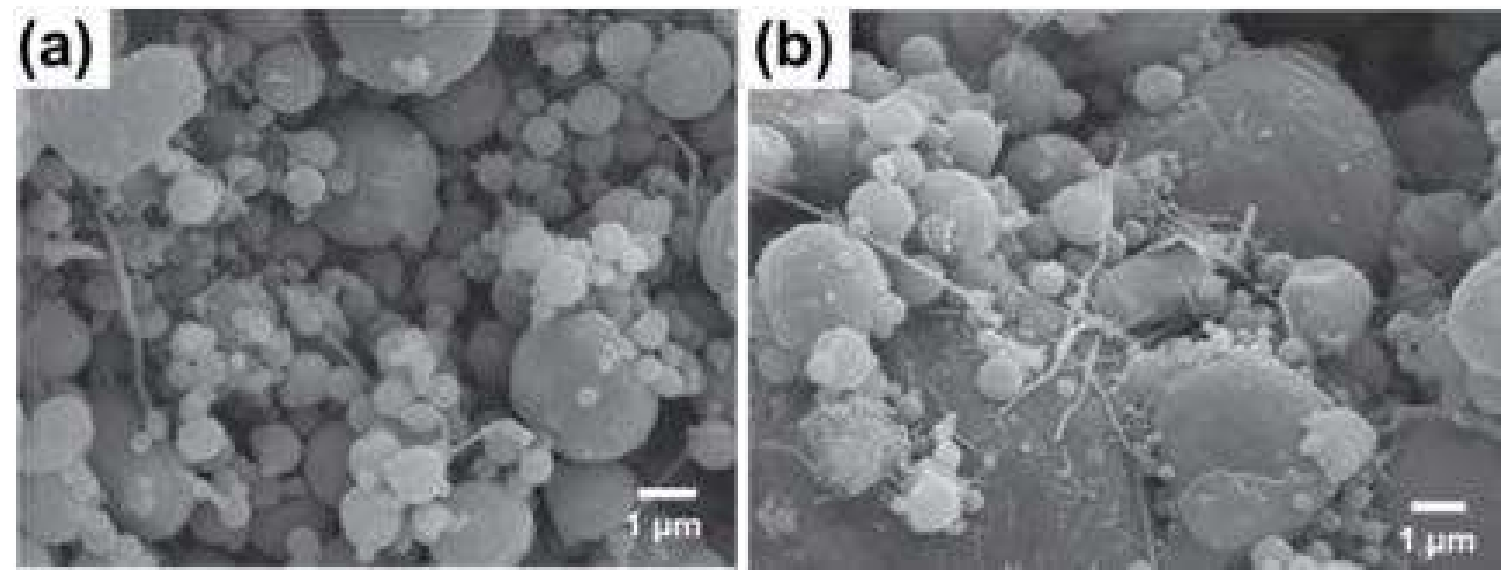

Figure 2.6. Al-BNNT composite powder prepared by wet-mixing: (a) 2 wt.\% BNNT, and (b) 5 wt. $\% \mathrm{BNNT}^{11}$.

\section{A. Wet-Mixing Method}

In wet-mixing approach, the nanotubes are first added to a liquid medium, such as acetone ${ }^{11}$ or ethanol ${ }^{12}$, and subjected to ultrasonication to deagglomerate and disperse the BNNTs. The studies employing wet-mixing method report the variation in sonication time from $1 \mathrm{~h}$ to $3 \mathrm{~h}^{11-13}$. Longer sonication helps in breaking BNNT clusters, which is important to engineer composites. However, excessive sonication can damage the nanotubes and cause shortening of their length, which is not desirable from the standpoint of mechanical reinforcement. Therefore, it is important to identify a fine balance, which can be done by 
imaging the composite mixtures after sonication and before composite manufacturing (Figure 2.6).

The BNNT dispersion step is followed by the addition of metal powder to the suspension. The metal-BNNT mixing is promoted by further sonication ${ }^{11}$, stirring $^{12}$ or by using a mechanical mixer ${ }^{14}$. Thereafter, the solvent is removed by evaporation.

\section{B. BNNT Functionalization}

Van der Waals forces acting between the nanotubes are responsible for their spontaneous clustering. Attaching a functional group to the nanotube surface can reduce the attraction forces, enabling their dispersion. Srivastava and co-workers used ball milling approach to attach amide and amine groups to the BNNTs ${ }^{13}$. This was accomplished by a four-step process:

(i) Mixing of $\mathrm{BNNT}$ and $\mathrm{NH}_{4} \mathrm{HCO}_{3}$ in acetone by ultrasonication,

(ii) Drying of the mixture at $40^{\circ} \mathrm{C}$ with simultaneous magnetic stirring,

(iii) Ball milling of the dried mixture at a speed of $323 \mathrm{rpm}$ for $2 \mathrm{~h}$ (using $\mathrm{ZrO}_{2}$ balls) and

(iv) Removal of the residual gases by heating the mixture (at $80^{\circ} \mathrm{C}$ in vacuum).

The authors mixed the functionalized BNNTs with Al powder by ultrasonication in ethanol (for 3 hours), followed by drying at $80^{\circ} \mathrm{C}$ while magnetically stirring the suspension. 

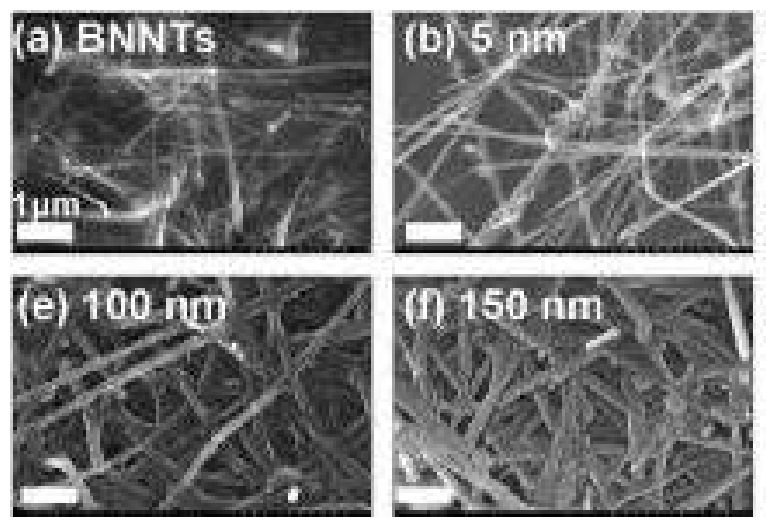
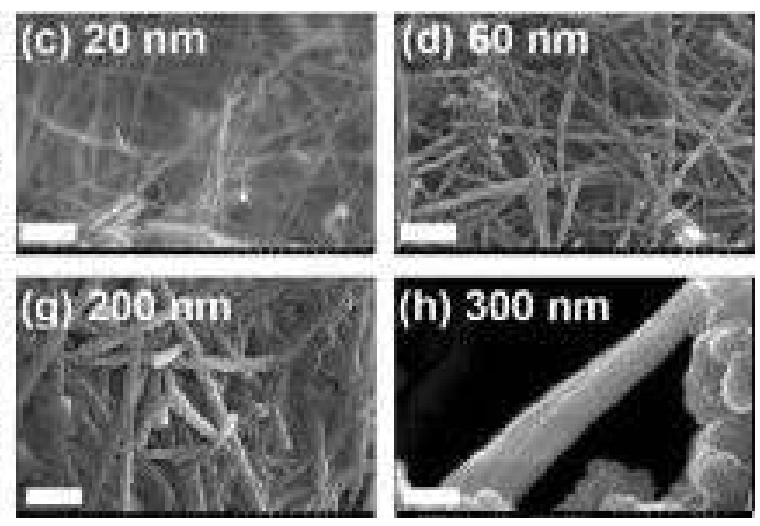

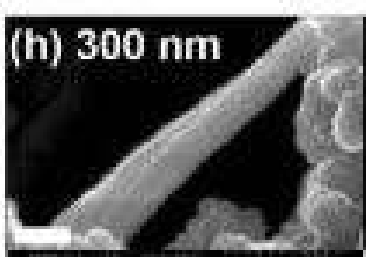

Figure 2.7. SEM micrographs showing variable Al coating thickness on BNNT (by varying the sputtering time) ${ }^{15}$.

\subsubsection{Manufacturing Approaches}

\section{A. Magnetron Sputtering}

Sputtering is a thin film deposition technique, which involves interactions between plasma, target material and substrate material inside a vacuum chamber. Plasma is created by ionizing an inert gas (such as Ar, Xe) and these ions are bombarded onto the target material (attached to the cathode). The energy transfer during bombardment causes the ejection of the target material particles. These escaped particles then travel towards the anode where the substrate is placed and get deposited onto the substrate surface. Yamaguchi et al. synthesized Al-BNNT hybrids by magnetron sputtering approach, using pure Al target (99.99\%) and BNNT (placed on $\mathrm{Si}$ wafer/ $\mathrm{Cu}$ grid/ $\mathrm{Au}$ microwire) as the substrate material $^{15}$. The study reported the use of Ar as the sputtering gas (flow rate of $20 \mathrm{sccm}$ ), the gas pressure of $500 \mathrm{mPa}, 200 \mathrm{~W}$ power, $11 \mathrm{~cm}$ standoff distance (between the substrate and the target) and a base pressure lower than $5 \times 10^{-4} \mathrm{~Pa}$. The thickness of the Al layer on BNNT was varied from $5 \mathrm{~nm}$ to $300 \mathrm{~nm}$ by tweaking the sputtering time (Figure 2.7). 
Bhuiyan and co-workers used sputtering approach to synthesize Ti-BNNT ${ }^{16}$. The study reported $100 \mathrm{~W}$ power, 600 seconds sputtering time, 5 mTorr gas pressure and Ar flow rate of $30 \mathrm{sccm}$. The authors also investigated the effect of heat-treatment on interface characteristics of the sputtered composite structures.

\section{B. Metal Ion Implantation}

In the ion implantation technique, the ion species are accelerated towards the target material and impinge the target surface upon impact (Figure 2.8). Obraztsova and coworkers used Ni-based alloy, Al and Ti for engineering BNNT composites ${ }^{17}$. The study reported acceleration voltage ranging from $5-30 \mathrm{kV}$, ion current was varied between 5 and $30 \mathrm{~mA}$, and $10 \mathrm{~cm}$ standoff distance between the BNNT sample and the ion acceleration zone. The irradiation dose depends on these processing parameters and was varied in this study from a lower bound of $1.73 \times 10^{17}$ ions $/ \mathrm{cm}^{2}$ to a high of $4.5 \times 10^{17}$ ions $/ \mathrm{cm}^{2}$.

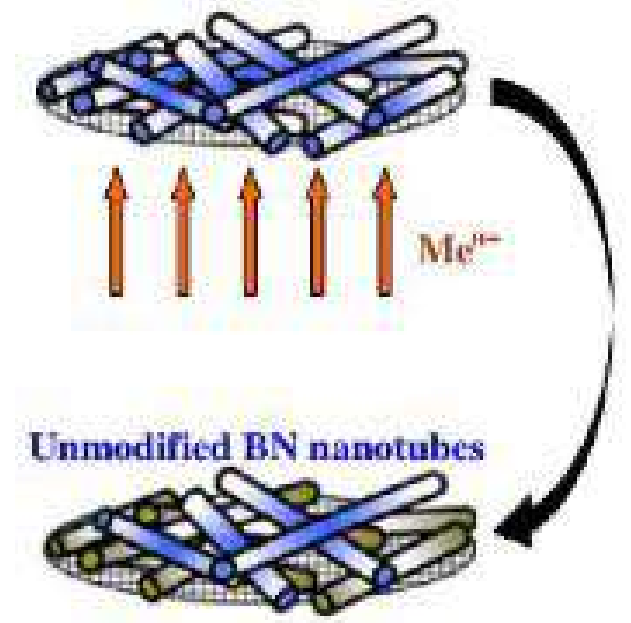

BN nanotuhes/Me

Figure 2.8. Metal ion implantation for fabricating BNNT-MMC ${ }^{17}$. 


\section{Melt Spinning}

Melt spinning is a solidification processing approach, in which liquid metal interfaces with a cooled rotating drum, producing solidified slender ribbons ${ }^{18}$. The technique has been employed for fabricating Al-BNNT composite ribbons ${ }^{14}$. In order to perform melt spinning, the authors first fabricated Al-BNNT pellets by compaction of composite powders at room temperature and $20 \mathrm{MPa}$ pressure. The pellets kept inside a quartz tube (with $1 \mathrm{~mm}$ diameter nozzle) were melted by induction currents, and melt spun on a water-cooled copper drum rotating with $24 \mathrm{~m} / \mathrm{s}$ speed. The fabrication was performed in Ar atmosphere. The schematic representation of the process and the ribbon output are shown in Figure 2.9.

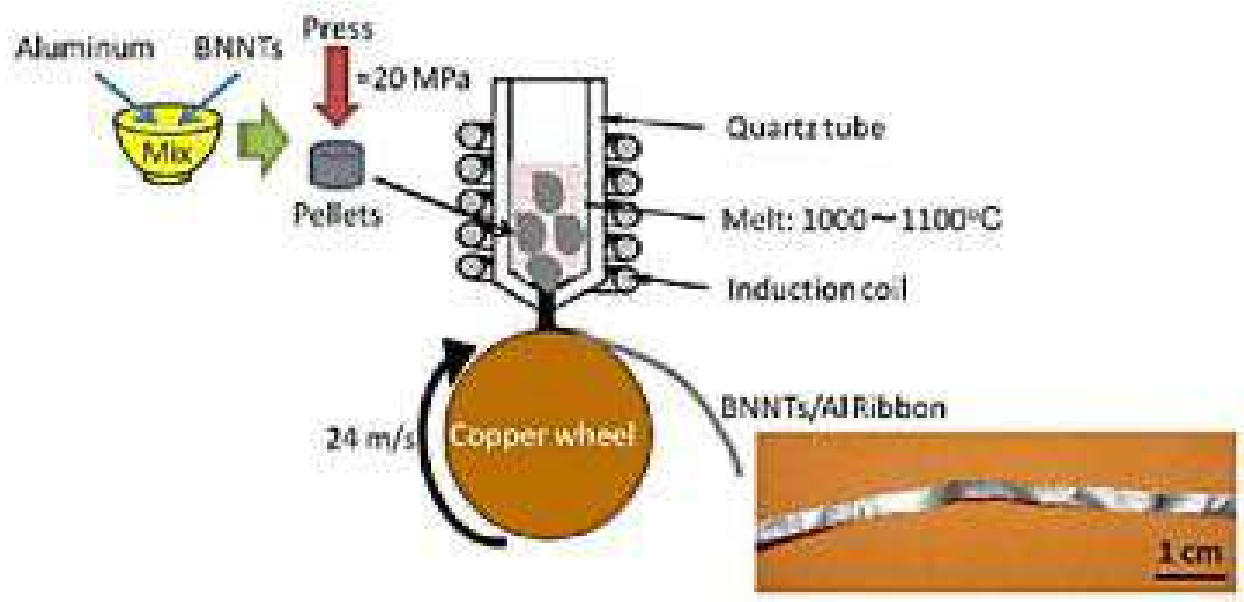

Figure 2.9. Schematic representation of the melt spinning process for fabricating AlBNNT ribbons ${ }^{14}$.

\section{Vacuum and Inert Gas Environment Sintering}

In the vacuum sintering approach, the powder is pressed in a die with desired shape and dimensions, and the green body is then sintered in a vacuum environment. Srivastava and co-workers used this approach for preparing a rectangular Al-BNNT green composite 
under 500-550 MPa pressure, which was sintered at $550^{\circ} \mathrm{C}$ for 3 hours ${ }^{13}$. The authors reported the furnace vacuum pressure used during the synthesis to be $\sim 10^{-2}$ Torr, and the heating and cooling rates of $20^{\circ} \mathrm{C} / \mathrm{min}$ and $10^{\circ} \mathrm{C} / \mathrm{min}$, respectively. In a relatively recent study, Bisht et al. sintered Al-BNNT in Ar gas environment ${ }^{19}$. The study reported relatively lower green body consolidation pressure of $200 \mathrm{MPa}$, higher sintering temperature of $600^{\circ} \mathrm{C}$, and shorter hold time of $1 \mathrm{~h}$. In addition, the sintered composites were subjected to post-sintering thermo-mechanical treatments: (i) warm rolling (60\% thickness reduction at $200^{\circ} \mathrm{C}$ ), followed by (ii) annealing at $350^{\circ} \mathrm{C}$ for $4 \mathrm{~h} . \quad \mathrm{A} \quad$ study on Ti-BNNT composite utilized higher processing temperatures $\left(800-1100^{\circ} \mathrm{C}\right)$. Integration of BNNT in Ti requires much higher sintering temperatures that $\mathrm{Al}$ due to the difference in their melting points of Ti.

\section{E. Spark Plasma Sintering}

Spark Plasma Sintering (SPS) is a field-assisted consolidation technique, where sample heating is accompanied by compaction forces (Figure 2.10). Direct current (DC) passage through the sample is responsible for Joule heating, facilitating faster consolidation that conventional pressure-less sintering techniques. There are a couple of reports on SPS of Al-BNNT composites ${ }^{11,12}$. Lahiri et al. employed $500^{\circ} \mathrm{C}$ temperature, $80 \mathrm{MPa}$ pressure, 1 hour hold time (at peak temperature) and $50-60^{\circ} \mathrm{C} / \mathrm{min}$ heating rate ${ }^{11}$. The sintering was performed in a vacuum environment and graphite die. The study reports the relative density of Al-BNNT composite to be $97.8 \%$ of the theoretical density, which is $\sim 1.6 \%$ lower than the density achieved for $\mathrm{Al}$ pellets. The authors ascribed this difference to partial BNNT 
agglomeration in the composite sample. The attempt to sinter the composite using a high temperature of $600^{\circ} \mathrm{C}$ resulted in partial melting. In a separate study, somewhat lower pressure (50 MPa) and sintering time (15-20 minutes) were used, allowing the authors to sinter the composites at a higher temperature of $600^{\circ} \mathrm{C}$ without melting ${ }^{12}$. It is worthy of attention that the $\mathrm{Al}$ powder sizes in the two studies were different: the powder size used in the former study was approximately half the powder size used by the latter group ( 20 $\mu \mathrm{m})$. These differences influence the nanotube distribution in the sintered composite.

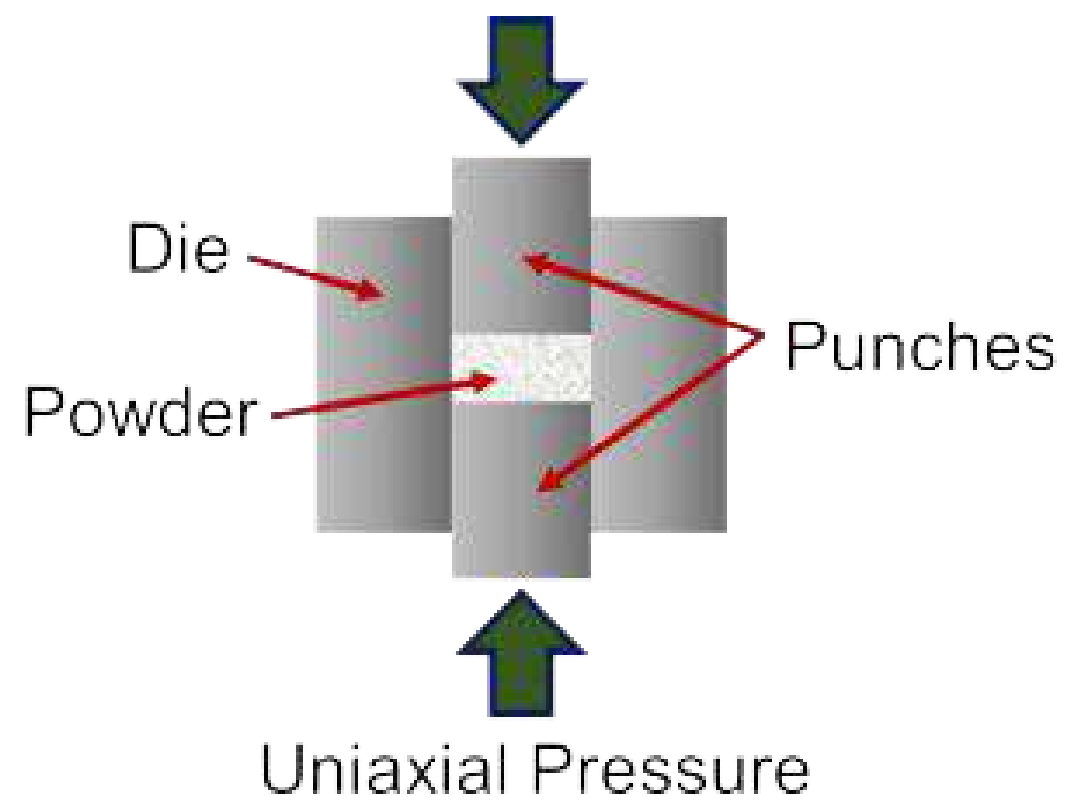

Figure 2.10. Schematic representation of spark plasma sintering for consolidating AlBNNT.

\section{F. High-Pressure Torsion}

The high-pressure torsion (HPT) technique relies on severe plastic deformation for powder consolidation. The powder is placed between two anvils, and there is a simultaneous application of extremely high pressure and mechanical strain (Figure 2.11). The pressure 
is introduced by pressing the two anvils, while the strain is introduced by rotating the anvils with respect to each other ${ }^{20}$. Yamaguchi et al. utilized HPT for consolidating Al-BNNT composite powder. The study reports compaction at room temperature, $2.5-5 \mathrm{GPa}$ pressure, 10 seconds - 5 minutes of pre-rotation pressure application, followed by 10 turns at $1 \mathrm{rpm}$ rotation speed ${ }^{12}$.

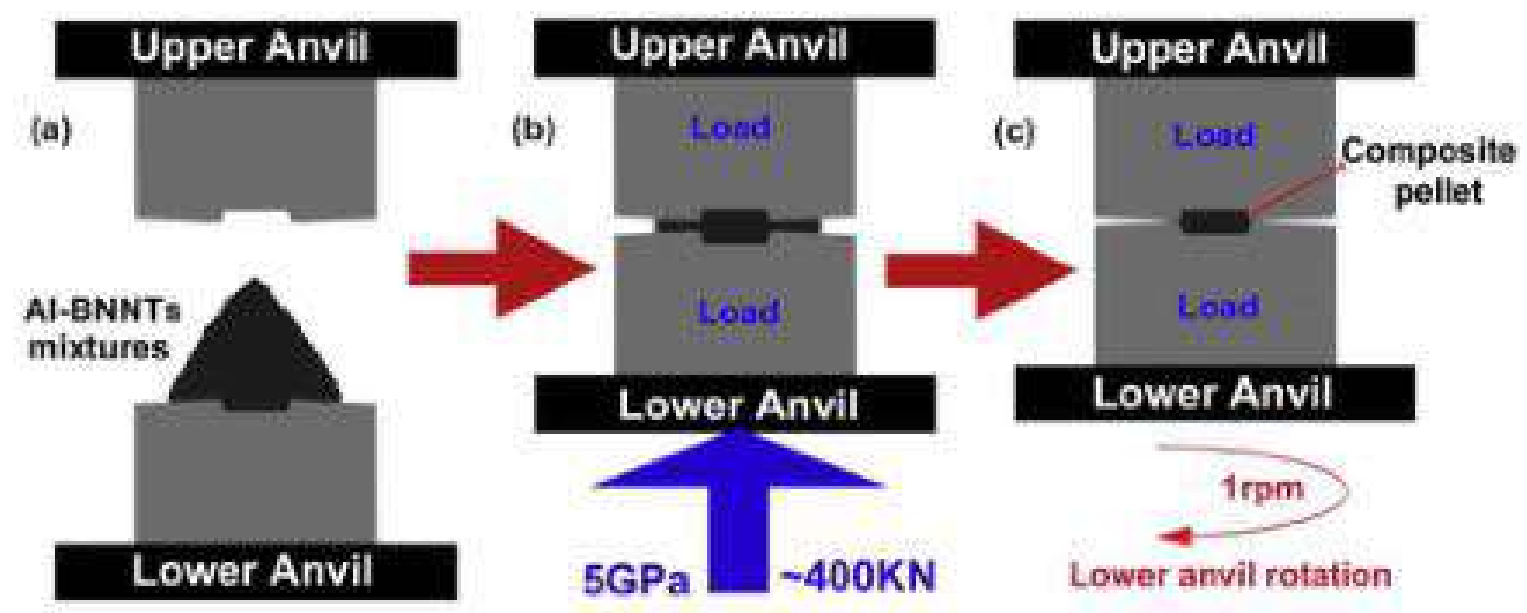

Figure 2.11. Schematic representation of high pressure-torsion consolidation of Al$\mathrm{BNNT}^{21}$.

\subsubsection{Critique}

Scalable manufacturing is vital for the commercial application of novel high-performance composites. The early works on BNNT-MMCs made use of processing techniques, such as magnetron sputtering, high-pressure torsion (HPT), pressureless vacuum sintering, spark plasma sintering (SPS) and melt spinning (summarized in Table 2.1). Most of these techniques can produce a small volume of materials, with millimeter-sizes or smaller dimensions. Techniques such as SPS or HPT reported in the literature require somewhat complex equipment setup, skilled manpower and are not as widely used in the 
manufacturing industry. It is desirable to explore the development of BNNT-MMCs by other manufacturing approaches, especially those which are easily scalable. For instance, casting (metal solidification) is one of the most extensive and traditional manufacturing techniques. There is a lack of information on whether BNNT can survive the extreme thermal conditions in a molten metal pool, the nature and extent of chemical interactions between molten metal and nanotubes, and the microstructure characteristics in cast BNNTMMCs. In the last decade, interest in additive manufacturing (AM) has grown exponentially because layer-by-layer material build-up enables the fabrication of netshaped or near net-shaped structures. However, AM techniques are characterized by nonequilibrium conditions due to ultrafast cooling rates. The possibility, challenges and strategies for BNNT integration via AM processes are currently unknown. The current gap in the fundamental understanding of composite processing via scalable manufacturing processes is a major roadblock in the widespread adoption of BNNTs as structural reinforcement filler for metal matrices.

Table 2.1. Small volume fabrication of BNNT-MMCs: Manufacturing processes vis-à-vis sample dimensions reported ${ }^{11-15,21}$.

\begin{tabular}{|c|c|c|}
\hline BNNT-MMC & Processing Technique & $\begin{array}{l}\text { Sample Shape and } \\
\text { Dimensions }\end{array}$ \\
\hline Al-BNNT Pellet ${ }^{13}$ & $\begin{array}{l}\text { Pressureless Vacuum } \\
\text { Sintering }\end{array}$ & $\begin{array}{l}\text { Rectangular Pellet } \\
13 \times 7 \mathrm{~mm}\end{array}$ \\
\hline Al-BNNT Nanohybrids ${ }^{15}$ & Magnetron Sputtering & $\begin{array}{l}\text { Cylindrical Tube } \\
\text { Diameter }=45-250 \mathrm{~nm}\end{array}$ \\
\hline Al-BNNT Pellet ${ }^{11}$ & Spark Plasma Sintering & $\begin{array}{l}\text { Cylindrical Pellet } \\
\text { Diameter }=20 \mathrm{~mm} \\
\text { Thickness }=5 \mathrm{~mm}\end{array}$ \\
\hline
\end{tabular}




\begin{tabular}{|c|c|c|}
\hline & Cold Rolling & $\begin{array}{l}\text { Strips (75\% thickness } \\
\text { reduction) }\end{array}$ \\
\hline Al-BNNT Ribbon ${ }^{14}$ & Melt Spinning & $\begin{array}{l}\text { Ribbon } \\
\text { Width }=4-5 \mathrm{~nm} \\
\text { Thickness }=50 \mu \mathrm{m} \\
\text { Precise length is not } \\
\text { reported }\end{array}$ \\
\hline Al-BNNT Pellets ${ }^{12}$ & $\begin{array}{l}\text { Spark Plasma Sintering } \\
\text { High Pressure Torsion }\end{array}$ & $\begin{array}{l}\text { Cylindrical Pellets } \\
\text { Diameter }=10 \mathrm{~mm} \\
\text { Thickness } \sim 3 \mathrm{~mm} \\
\text { Diameter }=10 \mathrm{~mm} \\
\text { Thickness } \sim 500 \mu \mathrm{m}\end{array}$ \\
\hline Al-BNNT Pellet ${ }^{21}$ & High Pressure Torsion & $\begin{array}{l}\text { Cylindrical Pellets } \\
\text { Diameter }=10 \mathrm{~mm} \\
\text { Thickness } \sim 500 \mu \mathrm{m}\end{array}$ \\
\hline Al-BNNT Strips ${ }^{19}$ & $\begin{array}{l}\text { Sintering } \\
\text { Warm Rolling }\end{array}$ & $\begin{array}{l}\text { Cylindrical Pellets } \\
\text { Diameter }=10 \mathrm{~mm} \\
\text { Thickness }=2 \mathrm{~mm} \\
\text { Strips (60\% thickness } \\
\text { reduction) }\end{array}$ \\
\hline $\begin{array}{l}\text { Ti-BNNT } \\
\text { Nanostructures }{ }^{16}\end{array}$ & Magnetron Sputtering & $\begin{array}{l}\text { Cylindrical Tubes } \\
\text { Diameter }=30-100 \mathrm{~nm} \\
\text { Length } \sim \text { few } \mu \mathrm{m}\end{array}$ \\
\hline Ti-BNNT Pellets $^{22}$ & Sintering & $\begin{array}{l}\text { Cylindrical Pellets } \\
\text { Diameter }=6 \mathrm{~mm} \\
\text { Thickness }=9 \mathrm{~mm}\end{array}$ \\
\hline
\end{tabular}




\subsection{Microstructure of Boron Nitride Nanotube-Metal Matrix Composites}

\subsubsection{Study of Nanotube Integration and Dispersion in the Matrix}

\section{A. Sputter-Coated Nanocomposite Structures}

The sputtering process was effective for obtaining homogeneous Al coating on BNNT. This was confirmed by EDS mapping of the sample, showing uniform Al distribution (Figure 2.12a) ${ }^{15}$. TEM imaging indicated the Al coating as polycrystalline. The tubular architecture and crystallinity of BNNT were retained during sputter coating (Figure 2.12b). XPS analysis revealed peaks associated with A12p, B1s, N1s, and O1s on the AlBNNT nanohybrid surfaces (Figure 2.12c). This confirms that the integrity of B-N bonds in the nanotubes is unaffected due to sputtering. A peak at $74.8 \mathrm{eV}$ was detected, which is related to $\mathrm{Al}_{2} \mathrm{O}_{3}$, suggesting the oxidation of nanotubes during sputter deposition. The room temperature processing conditions prevented any chemical reactions between BNNT and Al, which are often observed at elevated temperatures. Bhuiyan and co-workers examined interfacial reactions in sputtered Ti-BNNT nanostructures by post-deposition heattreatment ${ }^{16}$. The study reported the formation of $\mathrm{TiB}_{2}$ crystals when the composites were heat-treated to $800^{\circ} \mathrm{C}$ for $30 \mathrm{~min}$. The reactions accelerated with time (reported up to $1 \mathrm{~h}$ ) and temperature (reported up to $1100^{\circ} \mathrm{C}$ ), enhancing the fraction of $\mathrm{TiB}_{2}$ as well as the forming an additional TiB phase. 

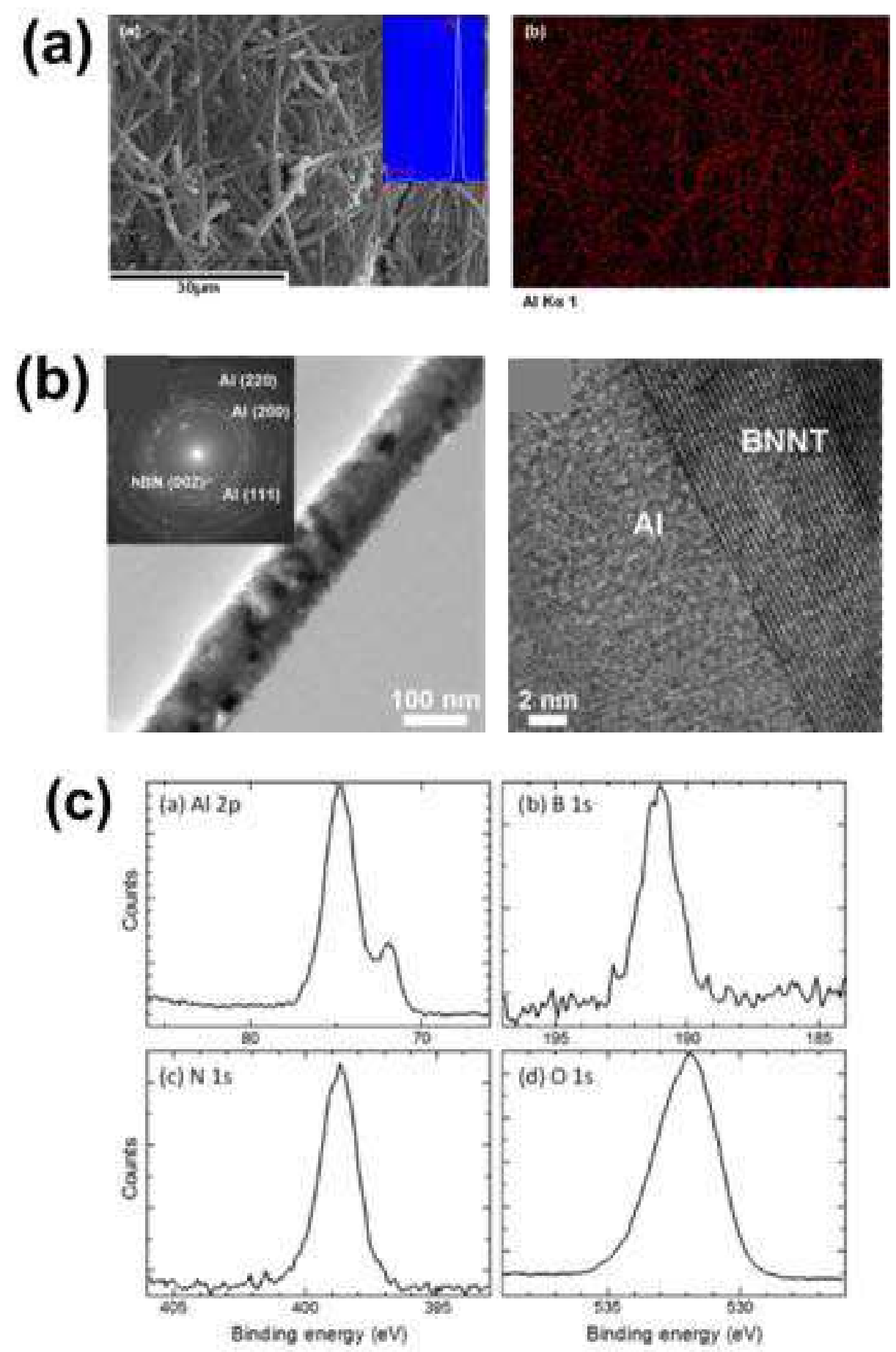

Figure 2.12. Microstructure assessment of sputter-coated Al-BNNT nanohybrids: (a) SEM image and the corresponding EDS map showing uniform coating of $\mathrm{Al}$, (b) TEM micrographs showing uniform coating of polycrystalline $\mathrm{Al}$ on BNNT, and (c) XPS spectra capturing $\mathrm{Al}, \mathrm{B}, \mathrm{N}$ and $\mathrm{O}^{15}$. 

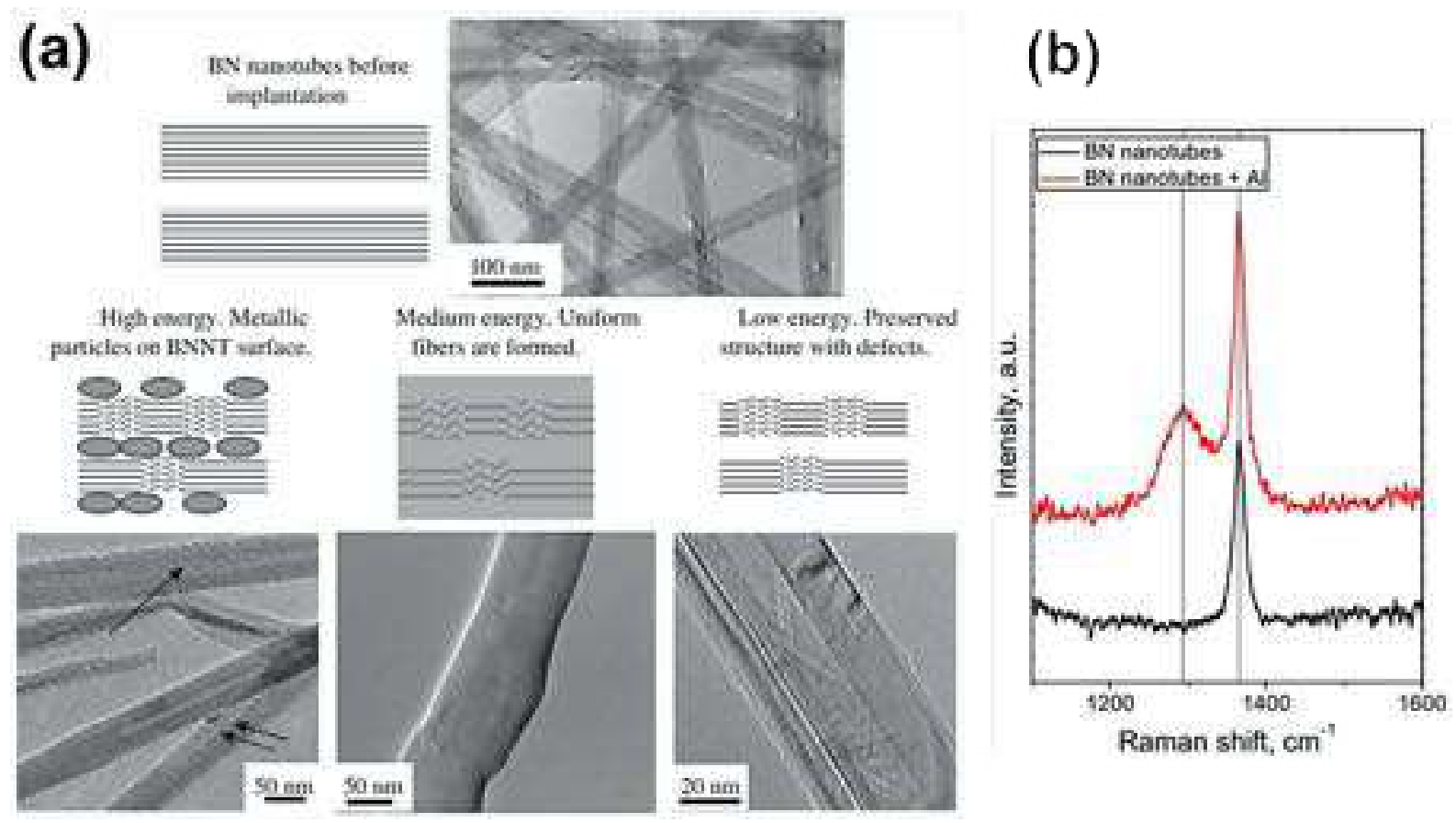

Figure 2.13. (a) Low-to-high energy regimes for metal ion implantation to fabricate BNNT-MMC structures, and (b) Raman spectra of pristine BNNT and Al-BNNT structures indicates amorphization due to ion implantation ${ }^{17}$.

\section{B. Metal Ion Implanted BNNT (Al, Ti and Ni-Alloy)}

It was observed that high energy metal ion implantation (at $30 \mathrm{kV}$ acceleration voltage) led to the localization of metal particles on the nanotube surface or hollow inner channel. Discrete metal particles, 5-10 nm size, were observed after implantation. On the other hand, relatively lower voltages $(10$ and $20 \mathrm{kV})$ were conducive to the formation of uniform coatings (Figure 2.13a). EDS analysis of the Al-BNNT structures revealed the presence of oxygen, indicating some degree of Al oxidation. Partial amorphization of the outer BNNT shell was observed due to metal implantation. The Raman characterization showed a downshifted broad feature $\left(1293 \mathrm{~cm}^{-1}\right)$ after implantation, in addition to the high-frequency mode $\left(1366 \mathrm{~cm}^{-1}\right)$. This observation confirmed the presence of crystalline as well as 
amorphous-like BN in the nanocomposite (Figure 2.13b). The authors concluded that lightweight $\mathrm{Al}$ implantation resulted in limited BNNT damage, as opposed to relatively heavier Ni and Ti metals. The study did not investigate or comment on the possible effects of amorphization on the mechanical properties of the composites.
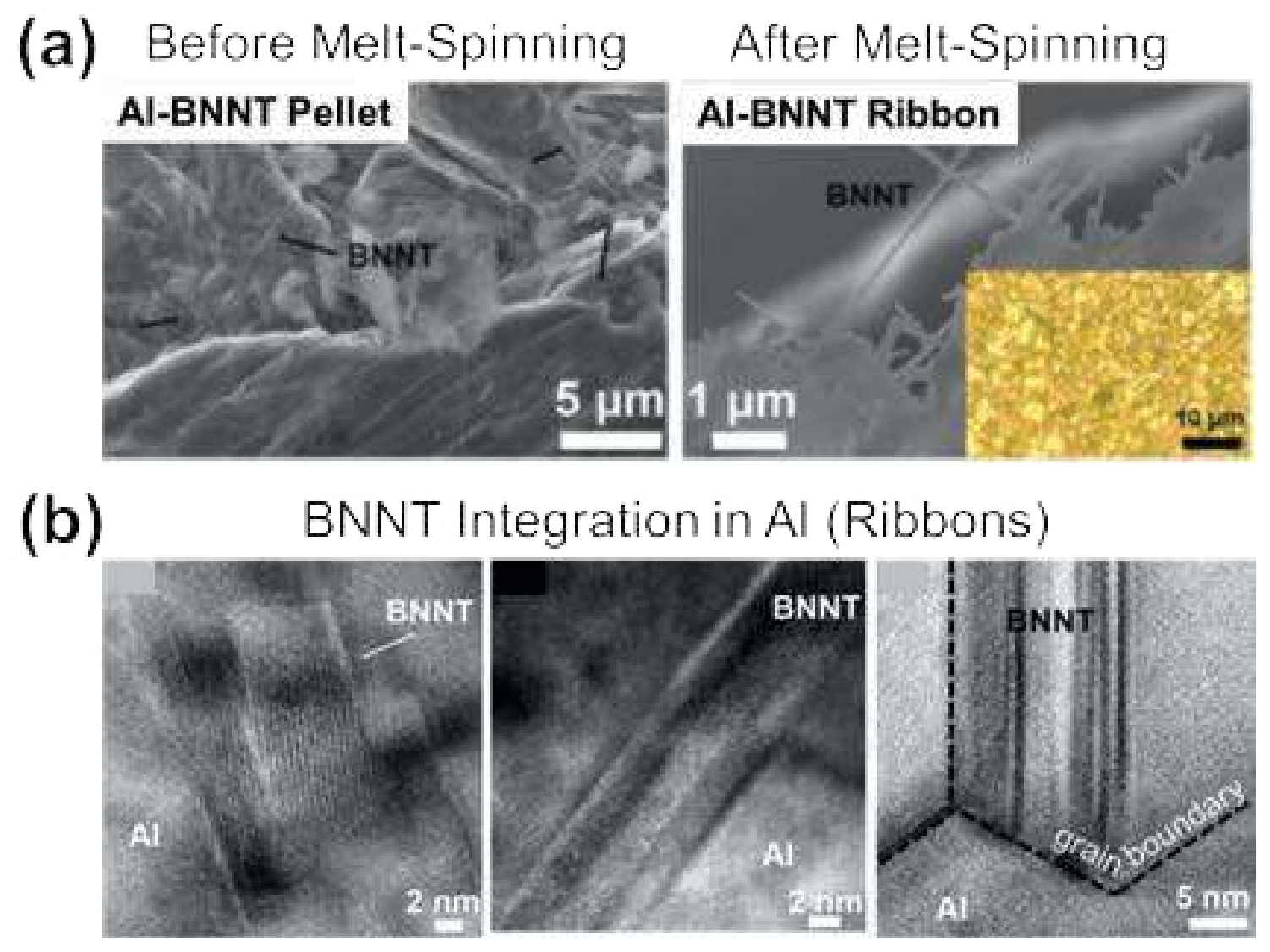

Figure 2.14. (a) SEM micrographs showing BNNT in Al pellet (before melt spinning) and Al ribbon (after melt spinning), and (b) HRTEM images showing the integration of BNNT in $\mathrm{Al}$ (inside grains as well as along grain boundaries) ${ }^{14}$.

\section{Melt-Spun Al-BNNT Composite}

The nanotubes in the melt-spun composite are randomly oriented. The grain size in the melt spun composites were reported to be extremely fine $\sim 2-3 \mu \mathrm{m}$ (inset to Figure 2.14a). TEM imaging revealed that BNNTs in the ribbon can be either inside the Al grains or they 
can be present along the grain boundaries (Figure 2.14b). No additional phases were detected, indicating there were no reactions between $\mathrm{Al}$ and BNNT. The authors observed that there was no strong alignment of nanotubes in the composite; at best, the BNNTs were quasi-oriented along the casting direction. It was mentioned that tuning melt viscosity by overheating may be helpful in achieving oriented nanotubes in the composite. However, there is a possibility of lightweight BNNTs floating to the top of the melt, which can be a major issue from the standpoint of homogeneous composite microstructure.

\section{Vacuum- and Inert Environment-Sintered Al-BNNT}

Al-BNNT composite fabricated by vacuum sintering was characterized by the presence of $\mathrm{AlN}$ and $\mathrm{AlB}_{2}$ reaction products (Figure 2.15). The sintering condition of $550^{\circ} \mathrm{C}$ and 3 hours of soak time was conducive to interfacial reactions between the nanotubes and the matrix. Despite these reactions, the structure and chemistry of BNNT were retained in the composite. The authors reported the presence of a 4-6 nm transition layer of $\mathrm{AlB}_{2}$ between $\mathrm{Al}$ and $\mathrm{BNNT}$. The formation of $\mathrm{AlB}_{2}$ was ascribed to the presence of amorphous $\mathrm{BN}$ nanoparticles on the BNNT surface. Additionally, $\mathrm{Al}_{2} \mathrm{O}_{3}$ was also detected in the composite by XRD. The densities of $\mathrm{AlB}_{2}, \mathrm{AlN}$ and $\mathrm{Al}_{2} \mathrm{O}_{3}$ are $3.19 \mathrm{~g} / \mathrm{cm}^{3}, 3.26 \mathrm{~g} / \mathrm{cm}^{3}$ and $3.9 \mathrm{~g} / \mathrm{cm}^{3}$, respectively. The formation of these higher density phases results in a net higher composite density $\left(\sim 2.5 \mathrm{~g} / \mathrm{cm}^{3}\right)$ than the theoretically predicted density of $\sim 2.4 \mathrm{~g} / \mathrm{cm}^{3}$ for Al-1.5 wt.\% BNNT. The presence of interphases is significantly enhanced in Ti-BNNT composite ${ }^{22}$. The formation of $\mathrm{TiB}$ phase was reported, and the reactions were exacerbated at elevated temperatures. All the BNNTs were consumed and transformed to TiB when sintering was performed at $1100^{\circ} \mathrm{C}$ for $3 \mathrm{~h}$. 

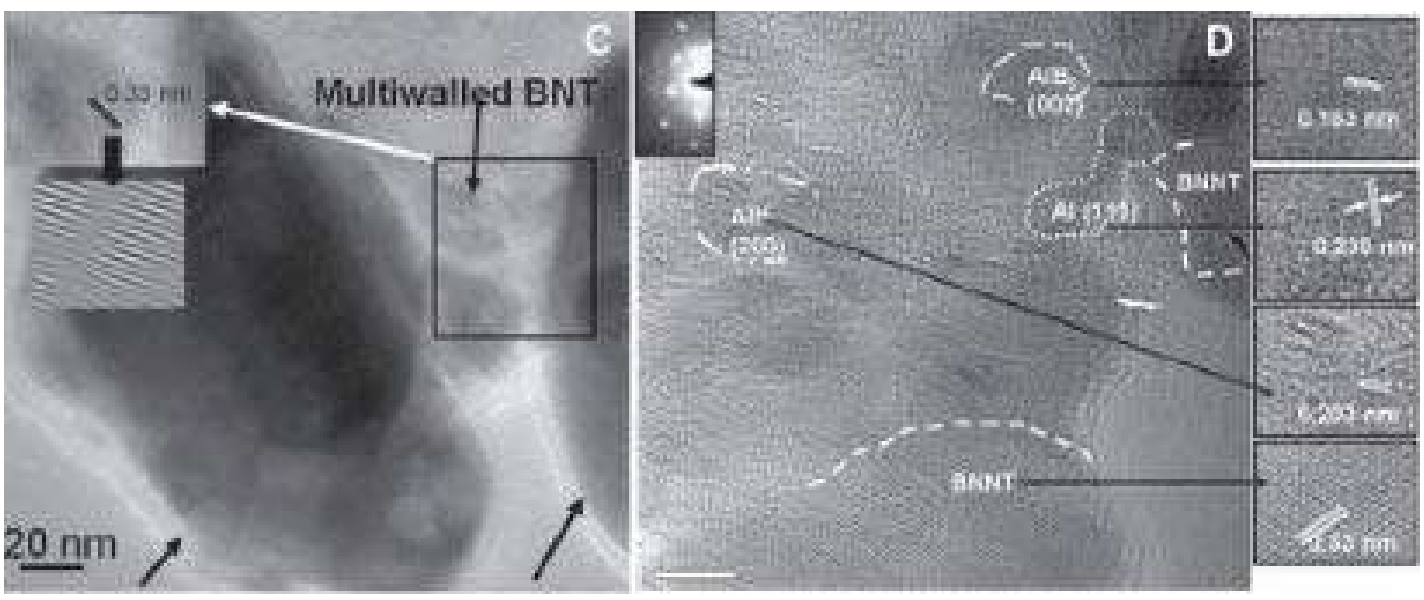

Figure 2.15. Formation of $\mathrm{AlN}$ and $\mathrm{AlB}_{2}$ due to interfacial reactions between $\mathrm{Al}$ and $\mathrm{BNNT}^{13}$.

Bisht et al. studied the effect of BNNT addition on grain size of $\mathrm{Al}$, and reported refinement and homogeneity in grain sizes in composite with 2 wt.\% BNNT ${ }^{19}$. This observation is ascribed to pinning of grain boundaries by nanotubes in the microstructure. Warm rolling of the pellets resulted in elongated grains, and the subsequent annealing step yielded fine-grained structure due to recrystallization. The authors also remarked that further addition of BNNT (to $5 \mathrm{wt} . \%$ ) led to agglomeration, porosity and non-homogeneous grain size distribution. The porous sample experienced cracking due to rolling, which underscores BNNT agglomeration can severely restrict workability of MMCs. The authors did not comment on interfacial reactions, which limits the understanding of microstructure evolution during hot working (rolling) and annealing of BNNT-Al composites.

\section{E. Spark Plasma Sintered Al-BNNT}

SPS consolidation has been reported for fabricating dense composites with minimal porosity. The fracture surface of the composite displayed the signature of ductile type 
failure, with thin ridges associated with localized necking (Figure 2.16a). High magnification SEM micrograph revealed the tubular BNNT protruding/ dangling from the fractured surface (Figure 2.16b). However, in a separate study conducted by Yamaguchi and co-workers, SPS failed to produce reasonable densification and bonding. BNNTs were seen to be located at the grain boundaries (Figure 2.16c). Prominent micropores/ holes were observed at the grain boundaries (Figure 2.16d). These differences in literature can be ascribed to different precursor powders and processing conditions adopted. The former study utilized finer sized Al powders $(\sim 10.5 \mu \mathrm{m})$, half the size employed by the latter (20 $\mu \mathrm{m})$. Smaller metal powder is expected to result in a homogeneous composite microstructure. Additionally, Yamaguchi et al. sintered the composite at a relatively lower pressure (50 MPa) and lower time (15-20 $\mathrm{min}$ ), whereas Lahiri and co-workers reported 80 MPa pressure and 1 hour hold time during sintering. These parameters play an important role in densification, explaining the observed differences in Figure 2.16 for the two studies. The lower time and pressure adopted by Yamaguchi et al. is understandable since they were sintering the composite at a relatively higher temperature of $600^{\circ} \mathrm{C}$ (as opposed to $500^{\circ} \mathrm{C}$ employed by Lahiri and co-workers). However, the findings seem to indicate this $100^{\circ} \mathrm{C}$ enhancement in temperature is not sufficient to balance the reduction in the pressure and time parameters.

The issue of BNNT accumulation at the boundaries is reflective dispersion challenges in Yamaguchi et al.'s work. Often, agglomeration is observed if the weight fraction of the nanofiller added to the matrix is too high. However, both of these studies report a similar range of BNNT weight percentage added to $\mathrm{Al}(1-5 \mathrm{wt}$. \%). Therefore, this difference in dispersion quality cannot be attributed to filler fraction. The issue then boils 

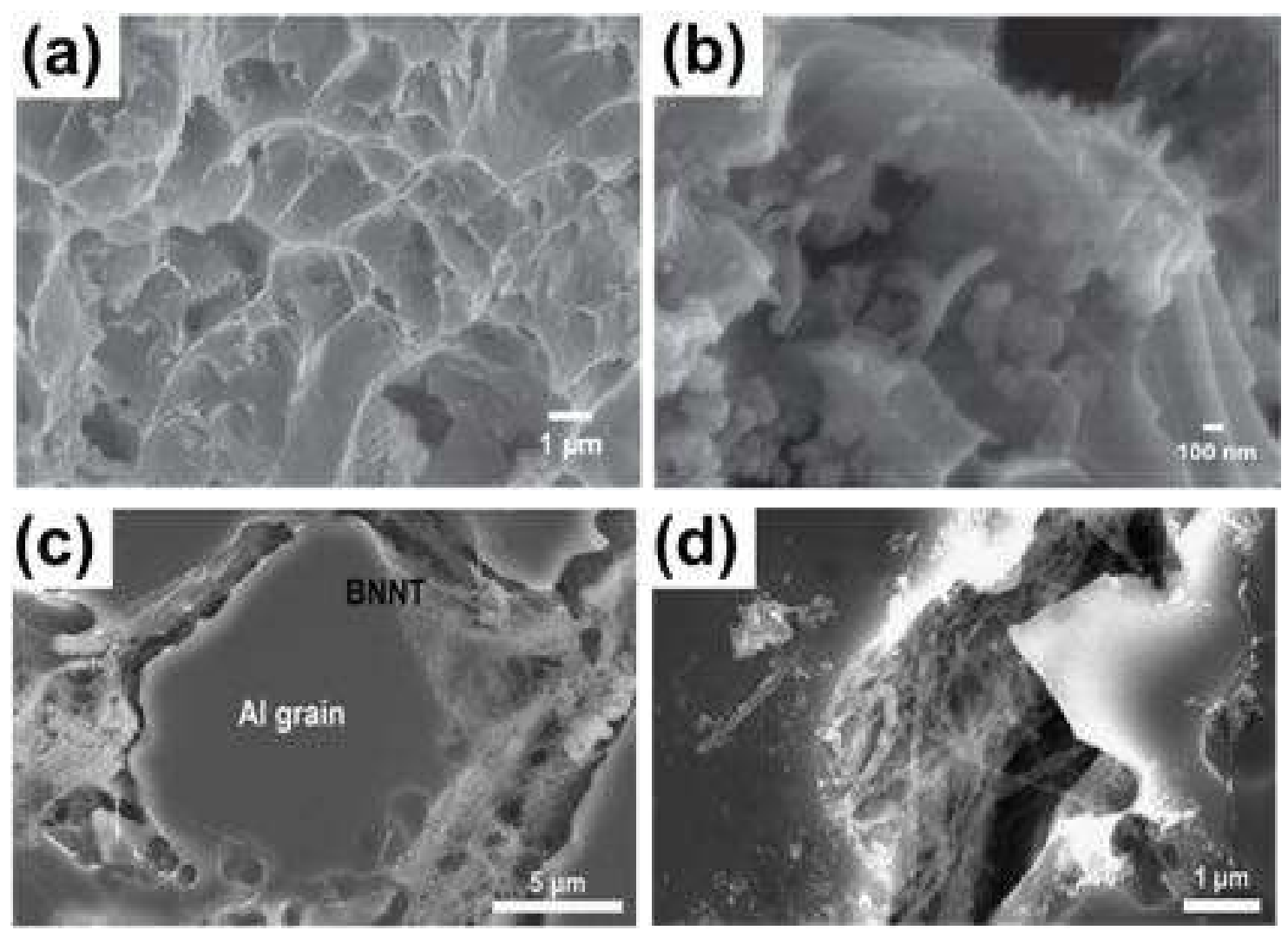

Figure 2.16. SEM micrographs of spark plasma sintered Al-BNNT showing: (a) signature of ductile failure ${ }^{11}$, (b) pull-out of nanotube ${ }^{11}$, (c) micro-porosity around grain boundaries $^{12}$, and (d) clustering of BNNT at grain boundaries ${ }^{12}$.

down to the first step of precursor powder preparation. Both studies employed the ultrasonication method for nanotube dispersion in liquid solvents. However, there is no quantification of the acoustic energy imparted to the solutions during sonication, which can impact the de-agglomeration efficiency. None of the studies comment on the concentration of BNNT in the liquid solvent (weight/volume) in this first stage of dispersion. Sonication may not be as effective if the BNNT loading in the solvent results in a highly concentrated suspension. Another point worth highlighting is that the method of metal powder introduction in the BNNT-solvent dispersion was somewhat different in the two studies: 
while Lahiri et al. continued to sonicate the suspension after metal powder addition, Yamaguchi and co-workers instead used magnetic stirring for mixing metal powders within the BNNT-solvent dispersion. It is not clear if this can produce significant differences in the homogeneity of precursor powders since only one of the studies provides a micrograph of the composite powders (Figure 2.6). Nevertheless, these observations are interesting and underscore the importance of dispersion for engineering homogeneous composite microstructures.

\section{F. High Pressure-Torsion-Consolidated Al-BNNT}

The grain size in HPT composites was significantly smaller (less than $1 \mu \mathrm{m}$ ), as compared to the composite prepared by SPS $(10-20 \mu \mathrm{m})$. TEM investigations revealed mixed success in BNNT integration: while some of the nanotubes were undamaged and retained their original state, several BNNTs experienced coagulation, loss of integrity, smashing, tearing, and bundling of due to heavy shear deformation in HPT (Figure 2.17a) ${ }^{12}$. Despite the physical transformations, there were no chemical reactions between $\mathrm{Al}$ and BNNT. The authors reported amorphous like layers in the interfacial region ${ }^{21}$. EELS signals indicated the presence of O-rich layer at the $\mathrm{Al} / \mathrm{BNNT}$ interface (Figure 2.17b). The physical degradation of BNNT reported in the study is concerning from the standpoint of mechanical reinforcement. Furthermore, the shearing of composite powder is not uniform during HPT: the regions close to the center experience lower strains than the outer periphery of the sample. This explains the difference in BNNT survival, deformation and integration reported in the study (Figure 2.17a). 

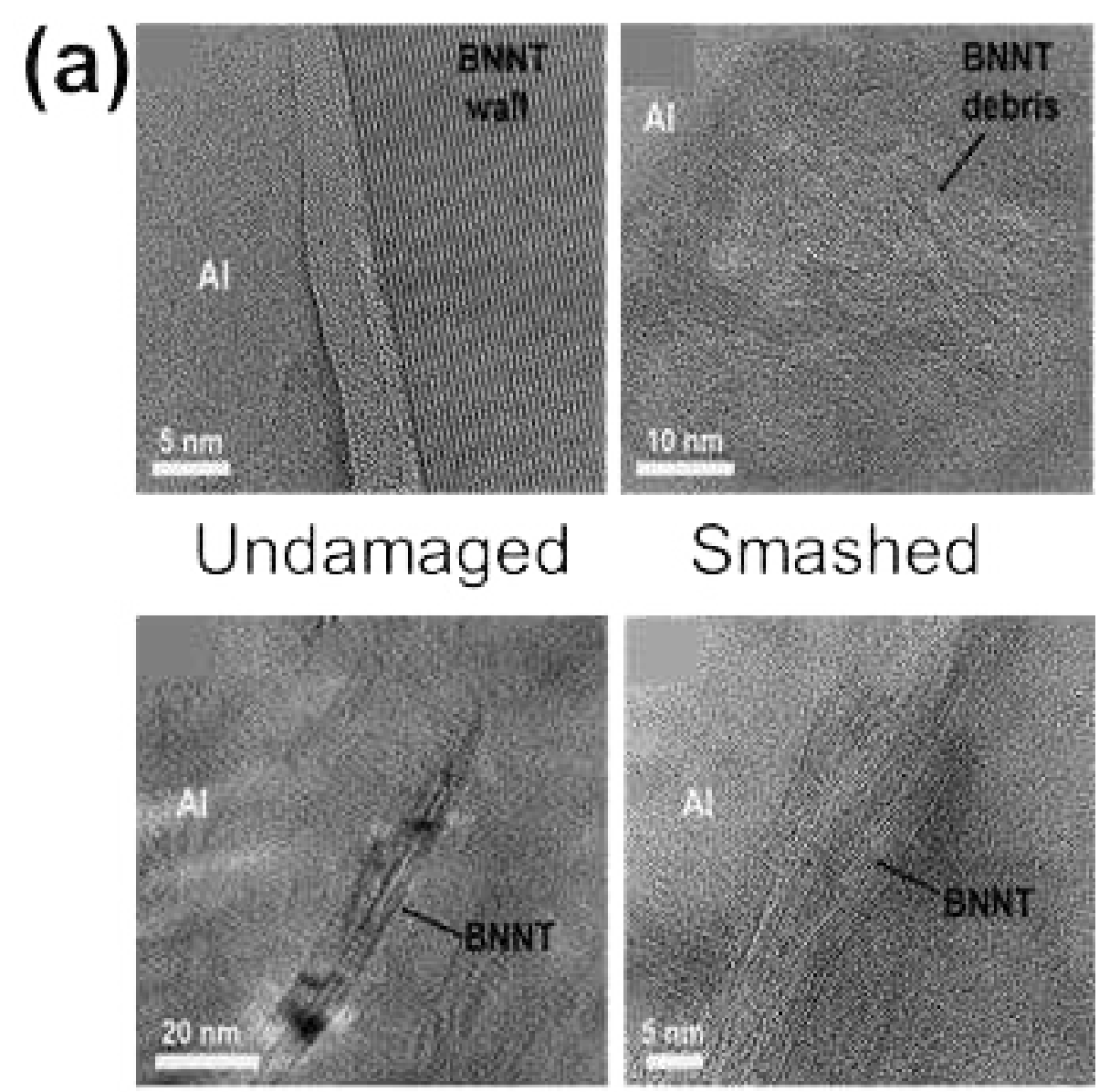

\section{Smashed}

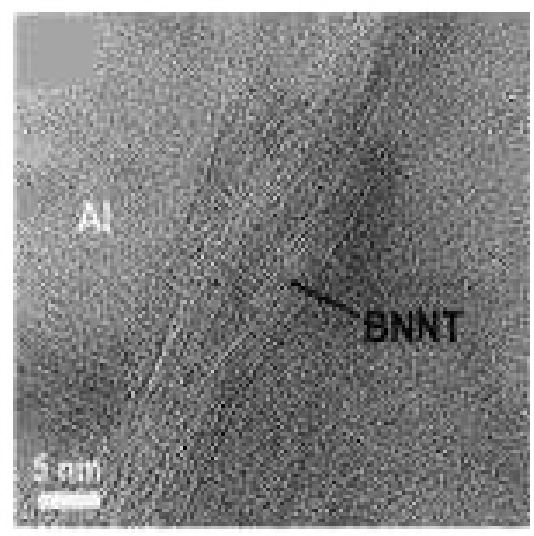

Flattened

\section{Torn}
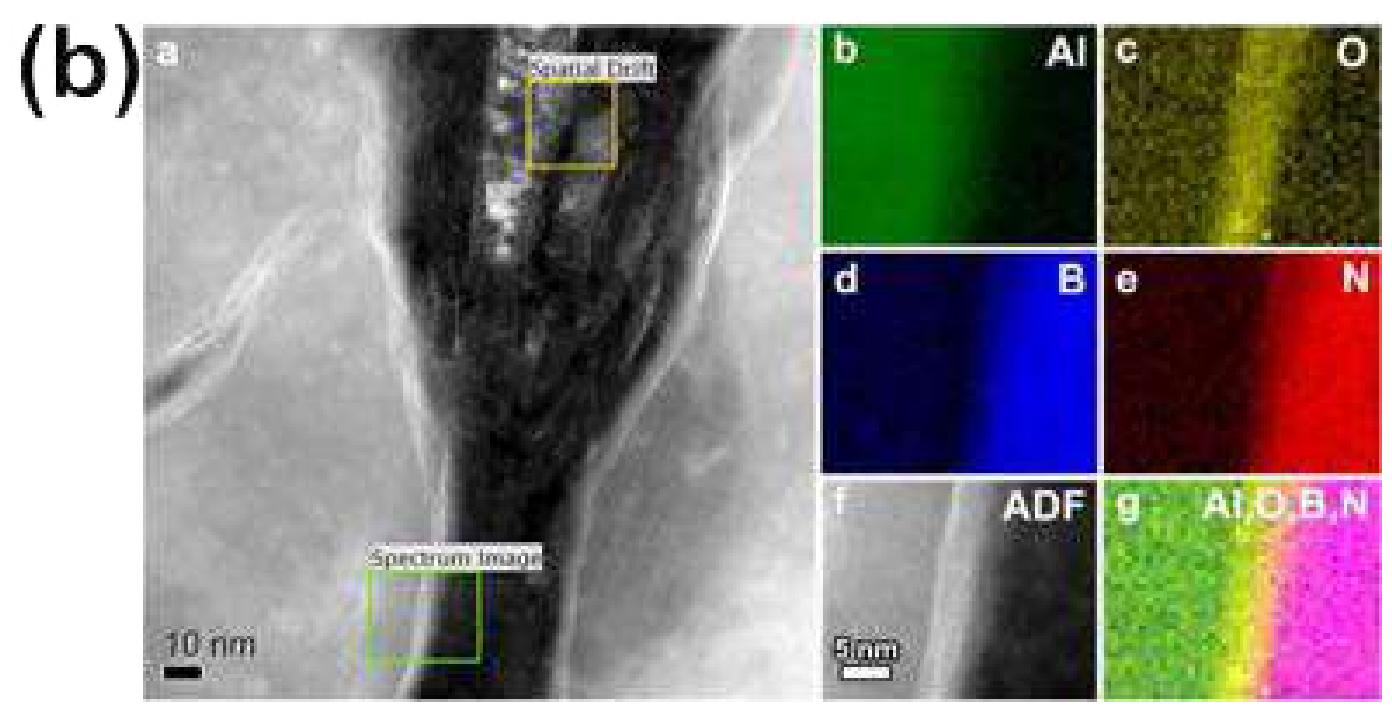

Figure 2.17. (a) Differences in BNNT retention/ integration ${ }^{12}$, and (b) EELS mapping of Al-BNNT interface in HPT-consolidated Al-BNNT composite ${ }^{21}$. 


\subsubsection{Critique}

\section{A. Nanotube Characteristics}

Strengthening in a composite is dictated by the stress-transfer from the matrix to the filler phase. For 1D nanotube reinforced composites, the tubes longer than a "critical length" are desirable for effective strengthening. This critical value $\left(l_{c}\right)$ corresponds to the scenario where the maximum stress at the center of the nanotube is equal to its intrinsic strength and is expressed by the relation ${ }^{23}$ :

$$
l_{c}=\frac{\sigma_{t} d}{\sigma_{m}}
$$

where $\sigma_{t}$ is the nanotube strength, $d$ is the nanotube diameter, and $\sigma_{m}$ is the matrix strength. Considering $\sigma_{t}=30 \mathrm{GPa}$ (for $\left.\mathrm{BNNT}\right)^{3}$ and $d=10 \mathrm{~nm}$, the critical length required for effective reinforcement of lightweight metals such as $\mathrm{Al}$ and $\mathrm{Mg}$ is calculated to be $7-15$ $\mu \mathrm{m}$. This critical length requirement can be three to six times higher if relatively thicker nanotubes (reported in the literature) are employed. Table 2.2 compares the morphologies, impurities and fabrication methods for the nanotubes used in some of the early works on Al-BNNT composite development (before 2015). It can be seen that these early studies relied on shorter nanotubes $(<10 \mu \mathrm{m})$ fabricated by mechanothermal, annealing and chemical vapor deposition techniques. Low aspect ratio nanotubes, such as the ones used in these works, do not provide effective strengthening and crack-bridging in the composite. Some of the studies also employ bamboo-shaped BNNTs, which do not have smooth morphology and are characterized by discontinuous lattices separated by joints. As a result, they display relatively inferior mechanical properties, with the experimentally measured 
fracture strength and Young's modulus $\sim 8 \mathrm{GPa}$ and $225 \mathrm{GPa}$, respectively. These values are roughly a quarter of the mechanical properties displayed by smooth, crystalline BNNTs. Use of short, impure and segmented nanotubes fails to realize the reinforcement potential of BNNTs.

Table 2.2 Fabrication method, morphology, dimensions, and impurities in BNNTs used for fabricating metal matrix composites reported in the literature.

\begin{tabular}{|c|c|c|c|}
\hline MMC & BNNT Fabrication & $\begin{array}{l}\text { BNNT Dimensions } \\
\text { (d: diameter, } l: \\
\text { length) }\end{array}$ & $\begin{array}{l}\text { Impurities in } \\
\text { the BNNT } \\
\text { filler }\end{array}$ \\
\hline Al-BNNT Pellet ${ }^{13}$ & $\begin{array}{l}\text { Mechanothermal } \\
\text { process (Ball milling } \\
\text { and annealing) }\end{array}$ & $\begin{array}{l}d=30-60 \mathrm{~nm} \\
l=0.5 \mu \mathrm{m}\end{array}$ & $\mathrm{O}, \mathrm{Cr}, \mathrm{Fe}$ \\
\hline $\begin{array}{l}\text { Al-BNNT } \\
\text { Nanohybrids }{ }^{15}\end{array}$ & $\begin{array}{l}\text { Boron oxide-assisted } \\
\text { CVD }\end{array}$ & $\begin{array}{l}d=40-50 \mathrm{~nm} \\
l=\text { Not } \text { specified }\end{array}$ & None reported \\
\hline Al-BNNT Pellet ${ }^{11}$ & $\begin{array}{l}\text { Ball milling and } \\
\text { annealing method }\end{array}$ & $\begin{array}{l}d=2-10 \mathrm{~nm} \\
l=3-5 \mu \mathrm{m}\end{array}$ & $\mathrm{O}, \mathrm{Fe}$ \\
\hline Al-BNNT Ribbon ${ }^{14}$ & $\begin{array}{l}\text { Boron oxide-assisted } \\
\text { CVD }\end{array}$ & $\begin{array}{l}d=40-50 \mathrm{~nm} \\
l=1-5 \mu \mathrm{m}\end{array}$ & None reported \\
\hline Al-BNNT Pellet ${ }^{12}$ & $\begin{array}{l}\text { Boron oxide-assisted } \\
\text { CVD }\end{array}$ & $\begin{array}{l}\text { Not specified } \\
\text { Cited Refs. Indicate: } \\
d \sim 50 \mathrm{~nm} \\
l \sim \text { up to } 10 \mu \mathrm{m}\end{array}$ & Impurity-free \\
\hline Al-BNNT Pellet ${ }^{21}$ & $\begin{array}{l}\text { Boron and metal } \\
\text { oxide-assisted CVD }\end{array}$ & $\begin{array}{l}\text { Not specified } \\
\text { Cited Refs. Indicate: } \\
d \sim 50 \mathrm{~nm} \\
l \sim \text { up to } 10 \mu \mathrm{m}\end{array}$ & Impurity-free \\
\hline Al-BNNT Strip ${ }^{19}$ & $\begin{array}{l}\text { Ball milling and } \\
\text { annealing method }\end{array}$ & $\begin{array}{l}d=2-10 \mathrm{~nm} \\
l=3-5 \mu \mathrm{m}\end{array}$ & $\mathrm{O}, \mathrm{Fe}$ \\
\hline $\begin{array}{l}\text { Ti-BNNT } \\
\text { Nanostructures }{ }^{16}\end{array}$ & $\begin{array}{l}\text { Ball milling and } \\
\text { annealing method }\end{array}$ & $\begin{array}{l}d=30-100 \mathrm{~nm} \\
l \sim \text { few } \mu \mathrm{m}\end{array}$ & $\mathrm{O}, \mathrm{Fe}$ \\
\hline
\end{tabular}




\begin{tabular}{|l|l|l|l|}
\hline Ti-BNNT Pellet & & & \\
\hline & Reactive ball milling & $\begin{array}{l}d=30-100 \mathrm{~nm} \\
l \sim a \text { few } \mu \mathrm{m}\end{array}$ & Iron Boride \\
\hline
\end{tabular}
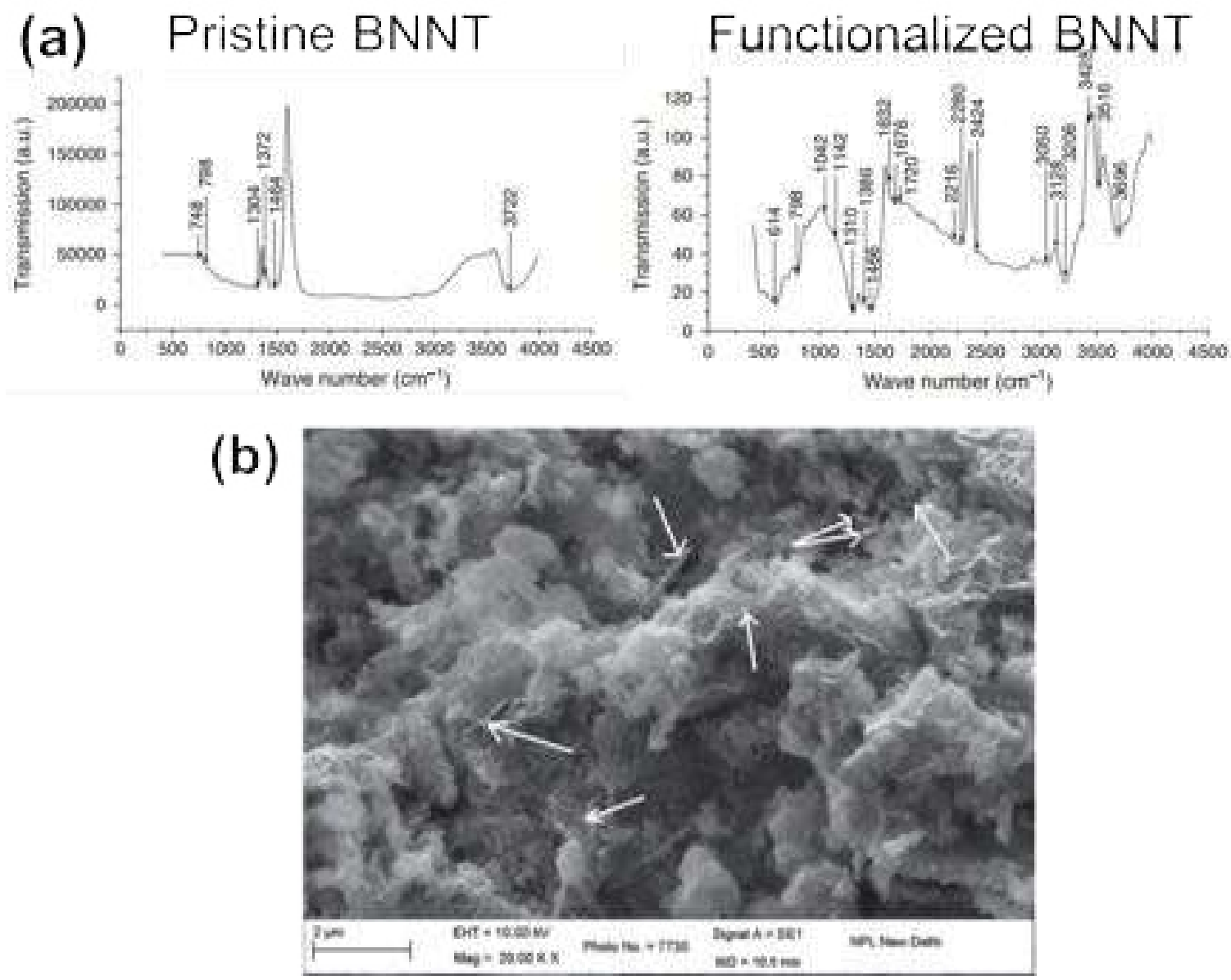

Figure 2.18. (a) Comparison of FTIR spectra for BNNT before and after functionalization, and (b) SEM micrograph of the fractured surface of Al-BNNT composite fabricated using amino functionalized nanotubes ${ }^{13}$.

\section{B. Effect of Functionalization}

One of the salient observations from the preceding discussion is that agglomeration is a major issue in the composites. The degree of clustering is seen to vary in different studies. 
Achieving the homogeneous distribution of nanotubes is vital for exploiting the mechanical characteristics of BNNTs. Srivastava et al. fabricated Al-BNNT composite fabricated using amino-functionalized nanotubes. The FTIR spectra of the functionalized BNNTs revealed additional absorption peaks when compared to the pristine nanotubes, confirming the attachment of functional groups (Figure 2.18a). The peaks corresponding to C-N stretching, $\mathrm{N}-\mathrm{H}$ bending, $\mathrm{N}-\mathrm{H}$ stretching and CO-NH were identified. SEM microscopy of the composite displayed homogeneous dispersion, and no significant clustering in the microstructure when functionalized BNNTs were employed (Figure 2.18b).

\subsection{Mechanical Properties of Boron Nitride Nanotube-Metal Matrix Composites}

\subsubsection{Microhardness}

Effect of BNNT addition on the microhardness has been explored for spark plasma sintered, high pressure-torsion consolidated, and vacuum/ inert environment sintered composites. Lahiri et al. reported a modest improvement in microhardness from $\sim 499 \mathrm{MPa}$ (Al) to $\sim 506 \mathrm{MPa}(5 \mathrm{wt} . \% \mathrm{BNNT})$ in spark plasma sintered pellets ${ }^{11}$. However, when the same samples were cold rolled, this difference between $\mathrm{Al}$ and $\mathrm{Al}-\mathrm{BNNT}$ widened as the composite hardness ( $807 \mathrm{MPa}$ ) was measured to be $\sim 25 \%$ higher than pure $\mathrm{Al}$ hardness (647 $\mathrm{MPa}$ ). The authors ascribed this improved reinforcement effect to possible densification and improved bonding due to rolling action. Contrary to these results, Yamaguchi and co-workers reported no improvement in microhardness of spark plasma sintered Al-BNNT composites ${ }^{12}$. As a matter of fact, there was some drop in hardness value for Al-5 wt.\% BNNT compared to pure Al. This deterioration in hardness was ascribed to 
micro-porosity along $\mathrm{Al}$ grain boundaries, which were the sites for BNNT clustering. These pores and agglomerates caused embrittlement of the samples, underscoring the criticality of dispersion for mechanical reinforcement. Notably, the authors reported enhanced microhardness when the HPT technique was used for consolidating the same Al-BNNT powder precursor. $5 \mathrm{wt} . \%$ BNNT addition resulted in more than two-times improvement in microhardness (190 MPa) as compared to pure $\mathrm{Al}$ sample $(90 \mathrm{MPa})^{12}$.
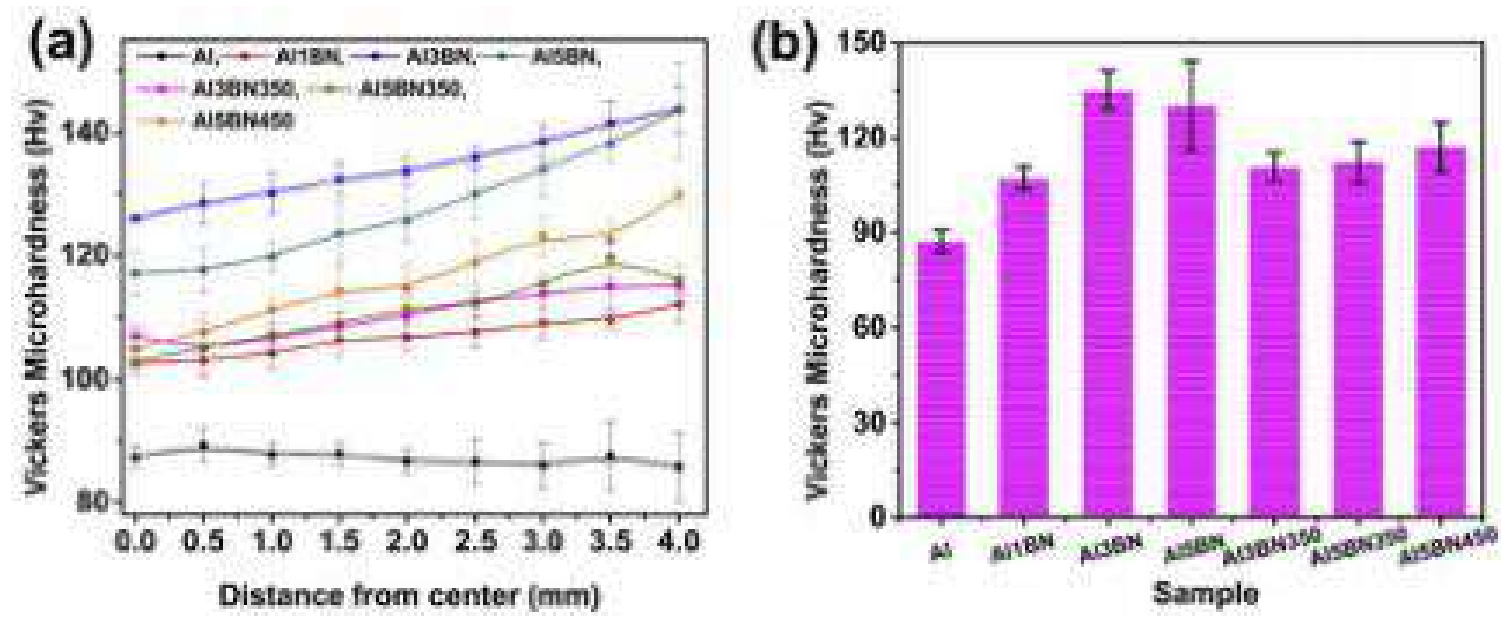

Figure 2.19. Microhardness of HPT-processed Al-BNNT: (a) Spatial variation from center to perimeter of the pellet, and (b) effect of BNNT volume fraction and heat-treatment (the numeral after Al indicates BNNT weight percentage and the numeral after BN indicates heat-treatment temperature) $)^{21}$.

In a separate study on HPT-processed composites, the spatial variation in microhardness was evaluated (for 1-5 wt.\% BNNT concentrations). The microhardness of Al-3 wt.\% BNNT was reported to be $\sim 1.5$ times the hardness of pure Al pellet. Moreover, the hardness values in the composite increased as the distance from the center of the pellet increased (Figure 2.19a). This variation is in striking contrast to pure Al, which displayed uniform hardness throughout the pellet. The spatial variation of hardness in Al-BNNT is 
ascribed to different degrees of local strains experienced by the disc (pellets) during HPT processing. As discussed in the preceding paragraph, severe deformation action (such as rolling or shearing) seem to enhance the density and bonding at metal-nanotube interfaces. Mechanical properties are greatly influenced by the quality of the filler/matrix interface. Since the pellet periphery experiences excess strains during HPT, the mechanical reinforcement is prominent away from the center.

The effect of heat-treatment on microhardness response of HPT-processed AlBNNT composites was examined by Xue and co-workers ${ }^{21}$. Heat-treatment at 350 and $450^{\circ} \mathrm{C}$ resulted in a reduction in microhardness by $\sim 18 \%$ and $\sim 13 \%$ respectively. The authors attributed this observation to microstructure recovery and associated events, such as recrystallization, rearrangement of dislocations, stress relief, movement of twin boundaries, and accommodation of grain boundary strains. Despite this reduction, the heattreated composites still exhibited higher microhardness as compared to pure Al pellets. Additionally, it was observed that the Al-BNNT composite treated at $450^{\circ} \mathrm{C}$ displayed somewhat higher hardness than the composites treated at $350^{\circ} \mathrm{C}$ (Figure $2.19 \mathrm{~b}$ ). The authors surmise if this marginal enhancement is due to new interfacial reactions, although there was no experimental investigation to evaluate such a possibility. Nevertheless, it should be noted that the selection of heat-treatment conditions should take into consideration the possibility of Al-BNNT reactions at elevated temperatures.

The most impressive improvement in microhardness was reported for amino functionalized BNNT-Al composite fabricated by vacuum (pressureless) sintering ${ }^{13}$. The authors compared the hardness of composites reinforced using pristine and functionalized 
BNNT. Addition of 1.5 wt. $\%$ pristine BNNT resulted in about $\sim 353 \%$ improvement in hardness from $32 \mathrm{~kg} / \mathrm{mm}^{2}$ to $145 \mathrm{~kg} / \mathrm{mm}^{2}$. When amino functionalized BNNTs were integrated in $\mathrm{Al}$, the hardness further improved to $180 \mathrm{~kg} / \mathrm{mm}^{2}$ (greater than five times the hardness of pure Al). Functionalization prevents agglomeration and helps in achieving uniform dispersion, which is desirable for mechanical reinforcement. It should also be noted that the vacuum sintered composite had additional ceramic phases, like AlN and $\mathrm{AlB}_{2}$ due to interfacial reactions. These products were not reported in SPS and HPTprocessed composites. Therefore, the improved microhardness is not merely because of BNNT reinforcement, but it is also a result of hard phases in the nanocomposite microstructure.

\subsubsection{Compression Strength}

The effect of BNNT addition on the compression behavior has been studied for spark plasma sintered and vacuum sintered composites. Lahiri et al. examined the micropillar compression response of spark plasma sintered Al-BNNT composite ${ }^{11}$. The study reported higher stress absorbance for Al-5 wt.\% BNNT (Figure 2.20a). A 54\% enhancement in yield strength was observed from $142 \mathrm{MPa}$ (for $\mathrm{Al}$ ) to $216 \mathrm{MPa}$ (for Al-BNNT). Fracture at $\sim 45^{\circ}$ angle (with respect to the loading direction) indicated slipping as the mode of deformation. BNNT bridges and sword in sheath structures were observed on the fracture surface of the micropillar (Figure 2.20b), attesting the activation of strengthening mechanisms associated with BNNT. While the initial slope of the curves suggests the stiffening of $\mathrm{Al}$ due to BNNT, the study did not report the actual values, citing concerns 
related to slippage during the test. The authors point out the possibility of inaccuracies in the strain values plotted in Figure 2.20a, making it difficult to compare and understand the effect of BNNT addition on the ductility of the composite.

A more remarkable augmentation in strength was reported by Srivastava and coworkers for amino functionalized BNNT-Al composite fabricated by pressureless vacuum sintering $^{13}$. Bulk-scale (macro) compression testing revealed three-fold enhancement (>250\% improvement) in compression strength from $117.4 \mathrm{~kg} / \mathrm{cm}^{2}$ to $419.11 \mathrm{~kg} / \mathrm{cm}^{2}$ by adding merely $1.5 \mathrm{wt} \%$ BNNT. However, the study did not provide the associated stressstrain curves to assess the elastic, plastic, and failure regimes. The reinforcement effect in this study is significantly higher than Lahiri et al.'s micropillar observations despite relatively lower BNNT content. There can be multiple reasons which can explain the differences:

(i) Functionalized BNNTs used in this report are easy to de-agglomerate and are uniformly dispersed in the composite microstructure as compared to pristine nanotubes employed by Lahiri et al.

(ii) The bulk-scale mechanical response captures the contribution of a large number of nanotubes; whereas micropillar response is localized and the test specimen volume can be devoid of sufficient nanotubes (especially if the dispersion is nonhomogeneous and the pillar dimensions are smaller than inter-nanotube spacing).

(iii) Srivastava et al. reported the formation of hard ceramic phases at the $\mathrm{Al} / \mathrm{BNNT}$ interface, which will also contribute towards strengthening of otherwise softer Al. There were no additional interfacial products reported by Lahiri et al. 


\section{(a)}

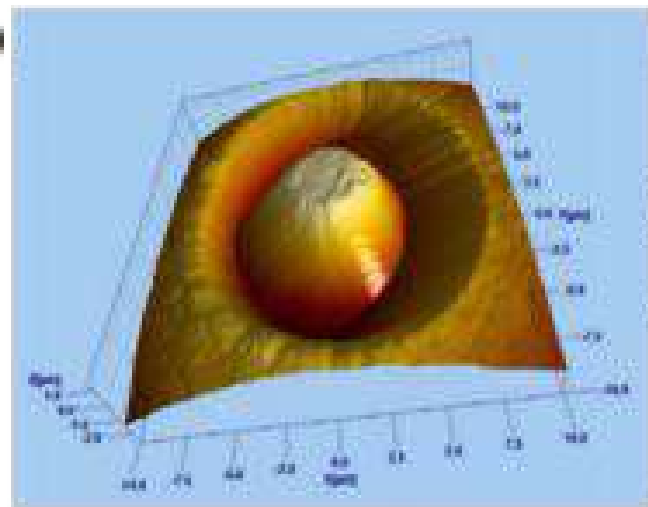

(b)

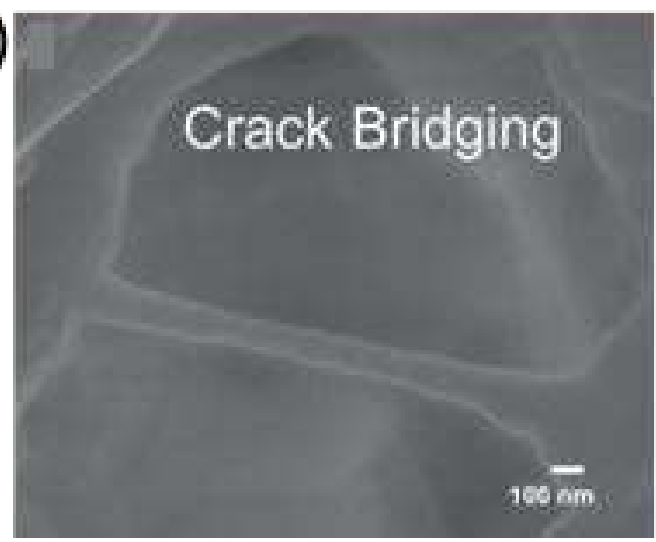

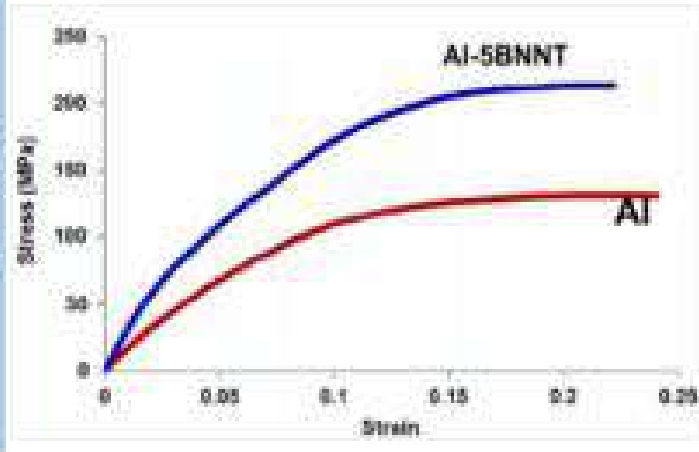

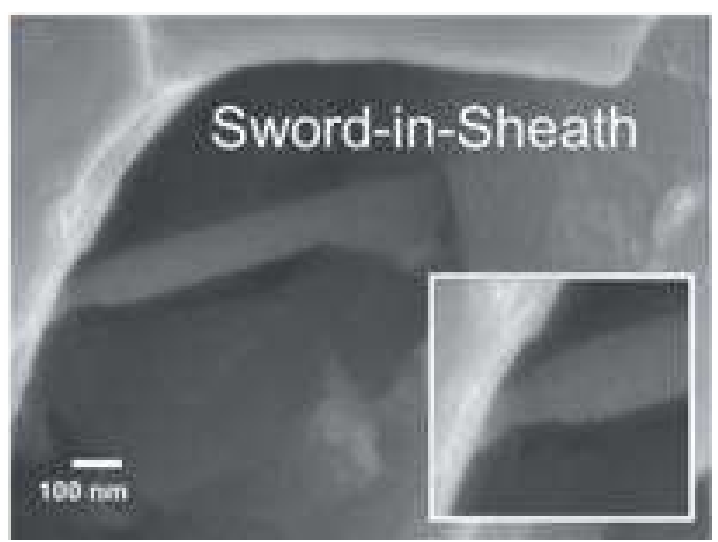

Figure 2.20. (a) Micropillar compression response of SPS-fabricated Al-BNNT composite, and (b) high magnification imaging of fracture mechanisms after pillar failure ${ }^{11}$.

Unlike Al-BNNT, the mechanical response of Ti-BNNT composites is strongly influenced by the interfacial reaction product in the microstructure ${ }^{22}$. The composites sintered at $1100^{\circ} \mathrm{C}$ displayed highest room temperature compressive strength (up to 1421 $\mathrm{MPa}$ ) as compared to the composites sintered at $800^{\circ} \mathrm{C}$, where the highest strength was found to be $984 \mathrm{MPa}$. The authors ascribed this finding to the formation of TiB interphase. The study also assessed high-temperature mechanical properties by performing compression at $500^{\circ} \mathrm{C}$. The same trend was observed in the high-temperature mechanical response: superior mechanical strength was recorded for composite with higher TiB 
content. The highest strength was recorded to be $413 \mathrm{MPa}$ for composite specimens sintered at $1100^{\circ} \mathrm{C}$, whereas it was $277 \mathrm{MPa}$ for the composite sintered at $800^{\circ} \mathrm{C}$. However, the composites sintered at $1100^{\circ} \mathrm{C}$ are arguably no longer Ti-BNNT due to the complete transformation of nanotubes. The composites fabricated at the intermediate temperature of $1000^{\circ} \mathrm{C}$ comprise of BNNTs with some degree of $\mathrm{TiB}$ formation at the interface, aiding in bonding between $\mathrm{Ti}$ and BNNT. The strength of these composites was observed to fall between the strengths of specimens with none $\left(800^{\circ} \mathrm{C}\right.$ sintering) or complete BNNT transformation $\left(1000^{\circ} \mathrm{C}\right.$ sintering). The study does not quantify the extent of reactions or secondary phase fractions in the microstructure. Therefore, the current understanding of interface chemistry-mechanics correlation in Ti-BNNT is limited.

\subsubsection{Tensile Strength}

Effect of BNNT addition on the tensile behavior has been explored for sputter-coated, melt spun, inert environment-sintered and high pressure-torsion consolidated composites. Yamaguchi et al. reported more than two times improvement in the ultimate tensile strength of melt spun ribbons (from $60 \mathrm{MPa}$ to $145 \mathrm{MPa}$ ) by adding up to $3 \mathrm{wt} \% \mathrm{BNNT}^{14}$. While the initial slope of the curves indicates an improvement in elastic modulus due to BNNTs; the absolute modulus values were rather low: $15 \mathrm{GPa}$ for $\mathrm{Al}$ and $35 \mathrm{GPa}$ for $\mathrm{Al}-3 \mathrm{wt} \%$ BNNT. These values are lower than the standard modulus for pure Al, and the authors suggest the discrepancy could be attributed to the microcrystalline nature of the sample or morphological features such as the presence of pores. However, there is no evidence presented in the study to substantiate these hypotheses. The fact that the strength values 
from the tests are within the typical range for $\mathrm{Al}$ weakens the argument attributing the lower moduli to microcrystallinity or porosity. It seems that the discrepancy in modulus values is not a material issue; rather it could be caused by sample slippage causing overestimation of strains. Slender samples, like ribbons with sub-mm thickness, are difficult to grip and may undergo slippage, a possibility that the authors have not considered. Addressing the problems with stiffness response of Al-BNNT composites is critical for structural applications.
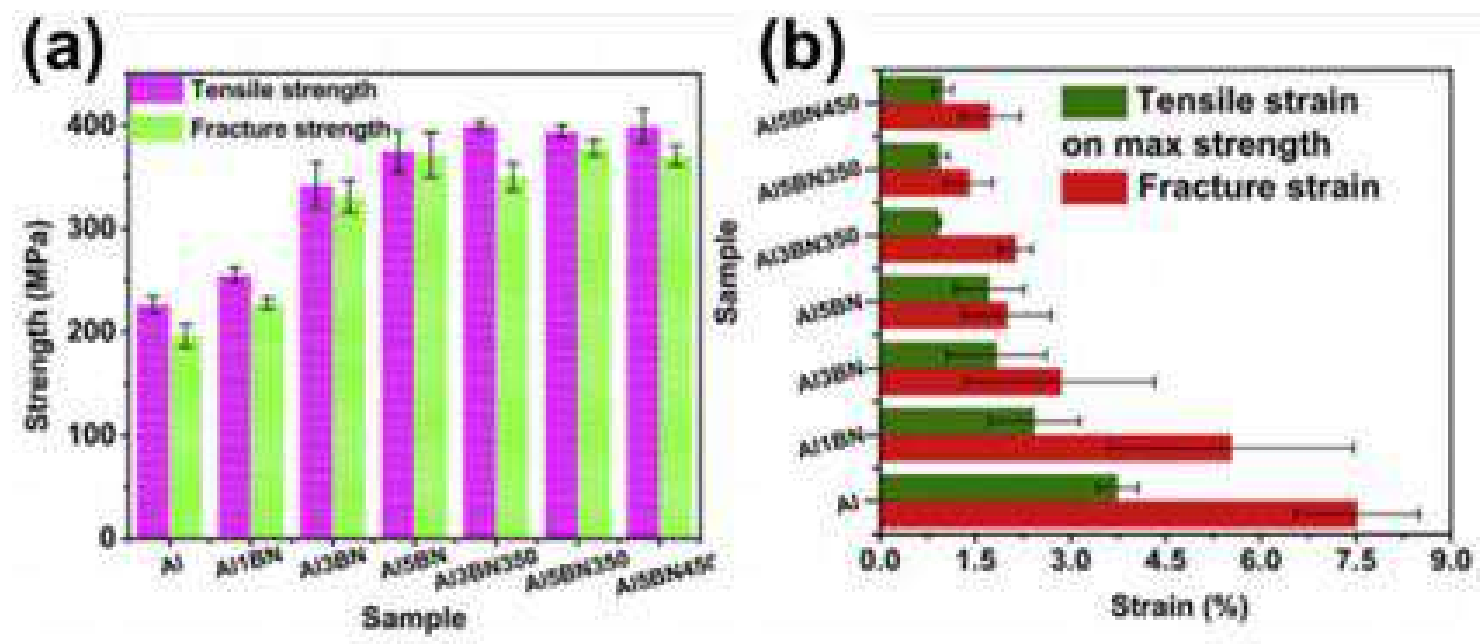

Figure 2.21. Effect of heat-treatment on tensile strength (a) and tensile strain (b) of HPTfabricated Al-BNNT composites ${ }^{21}$.

Al-BNNT strips fabricated by sintering and warm rolling demonstrated a modest $30 \%$ improvement in strength when 2 wt.\% BNNT was added ${ }^{19}$. An additional annealing step drastically enhanced the mechanical properties, as the composite with the same BNNT composition was characterized by $110 \%$ higher strength than Al. These differences are attributed to the restriction of grain growth and grain migration imposed by the presence of nanotubes in the microstructure. Relieving of internal stresses also improved the 
ductility of the composite from less than 0.006 (rolled composite) to about 0.01 (after annealing). These values are lower than $\mathrm{Al}$, which is expected in fiber-reinforced composites. However, even the absolute value of failure strain for $\mathrm{Al}$ is rather low (the maximum reported strain is about $1.1 \%$ for annealed specimens). Poor ductility will hamper the structural application of these materials. Nevertheless, the findings provide a glimpse into the possibility of programming mechanical properties of BNNT-MMCs by utilizing post-processing design space.

HPT-consolidated composite exhibited $\sim 1.5$ times higher tensile strength upon 3 wt.\% BNNT addition, as compared to Al. It was seen that $1 \mathrm{wt} . \%$ BNNT addition resulted in no change in the tensile strength, indicating a lack of effectiveness at lower filler fraction. However, there was an order of magnitude drop in strain to failure from $24 \%$ (pure $\mathrm{Al}$ ) to merely $6 \%$ (after $3 \mathrm{wt} . \%$ BNNT addition). The deterioration of plasticity was extreme for further addition of BNNT, highlighted by the authors' comment that the composite with 5 wt.\% BNNT could not be machined for making tensile samples due to brittleness. A separate study on HPT-fabricated composites further examined the effect of heat-treatment on the tensile response ${ }^{21}$. The ultimate tensile strength of HPT-processed composite improved by $70 \%$ when $5 \mathrm{wt} . \%$ BNNT was added to Al. Annealing the composite at 350 and $450^{\circ} \mathrm{C}$ resulted in a further $10 \%$ increase in the strength (Figure 2.21a). This observation is in stark contrast to the drop in microhardness after annealing reported in the same study and for the same heat-treatment conditions (Figure 2.19b). The authors attributed the strengthening behavior to the enhancement of Al-BNNT interfacial bonding. However, the hardness should also improve by the same rationale. The article does not address the opposite trends obtained for microhardness and tensile strength. Heat-treatment 
also resulted in the loss of ductility (Figure 2.21b). It was observed that the necking regime in heat-treated composites was extended, and the authors ascribed it to high temperature softening. This argument is contradictory to the reported reduction in failure strains after heat-treatment. If there was material softening, it should have manifested as enhanced ductility. Therefore, the claim of composite softening after heat-treatment may not be accurate and needs to be examined further. Nevertheless, the improvement in tensile strength of composites reported in all these studies is encouraging and supports the reinforcement potential of BNNT,

\subsubsection{Critique}

Some early studies have reported improvements in mechanical properties, such as hardness, compressive yield strength, elastic modulus and tensile strength of Al. However, the results are scattered and often not as striking as anticipated (summarized in Table 2.3). The low volume fraction of BNNT is possibly responsible for lackluster reinforcement. In order to achieve superb improvement in properties, the nanofiller addition should exceed the mechanical percolation threshold ${ }^{24,25}$. Mechanical percolation implies the formation of an interconnected network of nanofiller, which aids in superior stress-transfer. The percolation threshold depends on nanotube dimensions, and the theoretical relationship is given below:

$$
\phi_{c}=\frac{0.7}{l / d}
$$


where $l$ and $d$ represent nanotube length and diameter, respectively. This value is computed and compared with the actual volume fraction of nanotubes in all these BNNT-MMC reports (Table 2.3). Generally, the BNNT fraction seems to exceed the geometric percolation. However, the geometric percolation was determined based on the length and diameter of as-synthesized BNNTs. Nanotubes often experience structural degradation and shortening of length due to harsh physical conditions, such as long ultrasonication times, chemical action during functionalization, ball milling, mechanical mixing, shearing action, etc. Therefore, the actual length of BNNT within the composite is expected to be much shorter than the beginning nanotubes. Moreover, SEM and TEM investigations reported in these studies do not show the interconnected network of nanotubes, supporting the argument that the added BNNT volume fraction or the adopted dispersion techniques may not be sufficient to achieve percolation. Therefore, the efforts for MMC engineering should focus on interconnected microstructure design.

Table 2.3. Summary of mechanical reinforcement effect reported in the literature, the dimensions of nanotube employed, and a theoretical estimate of geometric percolation threshold for these reports.

\begin{tabular}{|c|c|c|c|c|}
\hline MMC & $\begin{array}{l}\text { BNNT } \\
\text { Fraction }\end{array}$ & $\begin{array}{l}\text { Mechanical } \\
\text { Properties } \\
\text { Reported }\end{array}$ & $\begin{array}{l}\text { BNNT } \\
\text { Dimensions } \\
(\text { d: diameter, l: } \\
\text { length })\end{array}$ & $\begin{array}{l}\text { Geometric } \\
\text { Percolation } \\
(\%)\end{array}$ \\
\hline Al-BNNT Pellet ${ }^{13}$ & $\begin{array}{l}1.5 \mathrm{wt} \% \\
\sim 2.9 \\
\text { vol } \%\end{array}$ & $\begin{array}{l}\text { Hardness } \\
\sim 275-462 \% \\
\text { improvement }\end{array}$ & $\begin{array}{l}d=30-60 \mathrm{~nm} \\
l=0.5 \mu \mathrm{m}\end{array}$ & 6.3 \\
\hline $\begin{array}{l}\text { Al-BNNT } \\
\text { Pellets }^{11}\end{array}$ & $5 \mathrm{vol} \%$ & $\begin{array}{l}\text { Compressive } \\
\text { Yield Strength }\end{array}$ & $\begin{array}{l}d=2-10 \mathrm{~nm} \\
l=3-5 \mu \mathrm{m}\end{array}$ & 0.11 \\
\hline
\end{tabular}




\begin{tabular}{|c|c|c|c|c|}
\hline & & $\begin{array}{l}50 \% \\
\text { improvement }\end{array}$ & & \\
\hline $\begin{array}{l}\text { Al-BNNT } \\
\text { Ribbons }{ }^{14}\end{array}$ & $\begin{array}{l}0.5-3 \\
\text { wt\% } \\
(\sim 0.9- \\
5.8 \text { vol } \\
\%)\end{array}$ & $\begin{array}{l}\text { Tensile Strength } \\
\text { Up to two times } \\
\text { improvement }\end{array}$ & $\begin{array}{l}d=40-50 \mathrm{~nm} \\
l=1-5 \mu \mathrm{m}\end{array}$ & 1.05 \\
\hline $\begin{array}{l}\text { Al-BNNT } \\
\text { Pellets }^{12} \\
\text { A. SPS }\end{array}$ & $\begin{array}{l}1-5 \mathrm{wt} \% \\
\text { (Up to } 9.7 \\
\text { vol \%) }\end{array}$ & Loss of hardness & $\begin{array}{l}\text { Not specified } \\
\text { Cited Refs. } \\
\text { Indicate: } \\
d \sim 50 \mathrm{~nm} \\
l \sim \text { up to } 10 \mu \mathrm{m}\end{array}$ & 0.35 \\
\hline B. HPT & & $\begin{array}{l}\text { Up to } 1.5 \text { times } \\
\text { improvement in } \\
\text { tensile strength }\end{array}$ & & \\
\hline $\begin{array}{l}\text { Al-BNNT } \\
\text { Pellets }^{21}\end{array}$ & $\begin{array}{l}1-5 \text { wt. } \% \\
\text { (Up to } 9.7 \\
\text { vol \%) }\end{array}$ & & & \\
\hline A. HPT & & $\begin{array}{l}70 \% \\
\text { improvement in } \\
\text { tensile strength }\end{array}$ & & \\
\hline $\begin{array}{l}\text { B. } \\
\text { HPT+Annealing }\end{array}$ & & $\begin{array}{l}\text { Further } 10 \% \\
\text { enhancement }\end{array}$ & & \\
\hline Al-BNNT Strips ${ }^{19}$ & $\begin{array}{l}2,5 \text { wt. } \% \\
\text { (Up to } 9.7 \\
\text { vol \%) }\end{array}$ & Tensile Strength & $\begin{array}{l}d=2-10 \mathrm{~nm} \\
l=3-5 \mu \mathrm{m}\end{array}$ & 0.11 \\
\hline $\begin{array}{l}\text { A. Sintering + } \\
\text { Rolling }\end{array}$ & & $\begin{array}{l}\text { Up to } 30 \% \\
\text { improvement }\end{array}$ & & \\
\hline $\begin{array}{l}\text { B. After } \\
\text { Annealing } \\
\text { Step }\end{array}$ & & $\begin{array}{l}\text { Up to } 110 \% \\
\text { strengthening }\end{array}$ & & \\
\hline $\begin{array}{l}\text { Ti-BNNT } \\
\text { Pellets }^{22}\end{array}$ & $\begin{array}{l}0.5-5 \text { wt. } \% \\
\text { (Up to } 9.7 \\
\text { vol. \%) }\end{array}$ & $\begin{array}{l}\text { Compressive } \\
\text { Strength }\end{array}$ & $\begin{array}{l}d=30-100 \mathrm{~nm} \\
l \sim a \text { few } \mu \mathrm{m}\end{array}$ & - \\
\hline
\end{tabular}




\begin{tabular}{|c|c|c|}
\hline & & $\begin{array}{l}\text { Room- } \\
\text { Temperature } \\
\text { Up to } 85 \% \\
\text { strengthening } \\
\text { At } 500^{\circ} \mathrm{C} \\
\text { Up to } 212 \% \\
\text { strengthening } \\
\text { (TiB-dominated } \\
\text { response) }\end{array}$ \\
\hline
\end{tabular}

\subsection{In-situ Investigation of Deformation Mechanisms Boron Nitride Nanotube- Metal Matrix Composites}

In-situ investigations are useful to unravel the deformation characteristics in nanocomposites, which have highly hierarchical microstructures. Time-resolved analysis of mechanisms throws light on the kinetics of composite deformation, which is not possible by post-mortem analysis.

\subsubsection{In-situ Tensile Deformation}

Yamaguchi and co-workers examined the tensile deformation of Al-BNNT nanohybrids inside TEM. The slender, 1D nanocomposites were fabricated by sputtering of a $40 \mathrm{~nm} \mathrm{Al}$ layer on BNNT. The study reported uniform stretching of the nanohybrid under tension, indicative of strong bonding and effective stress-transfer at the metal-nanotube interface (Figure 2.22). The deformation was purely elastic in the testing regime, which was limited by the instrument's upper force limit of $6 \mu \mathrm{N}$. This corresponded to $360 \mathrm{MPa}$ tensile stress, 

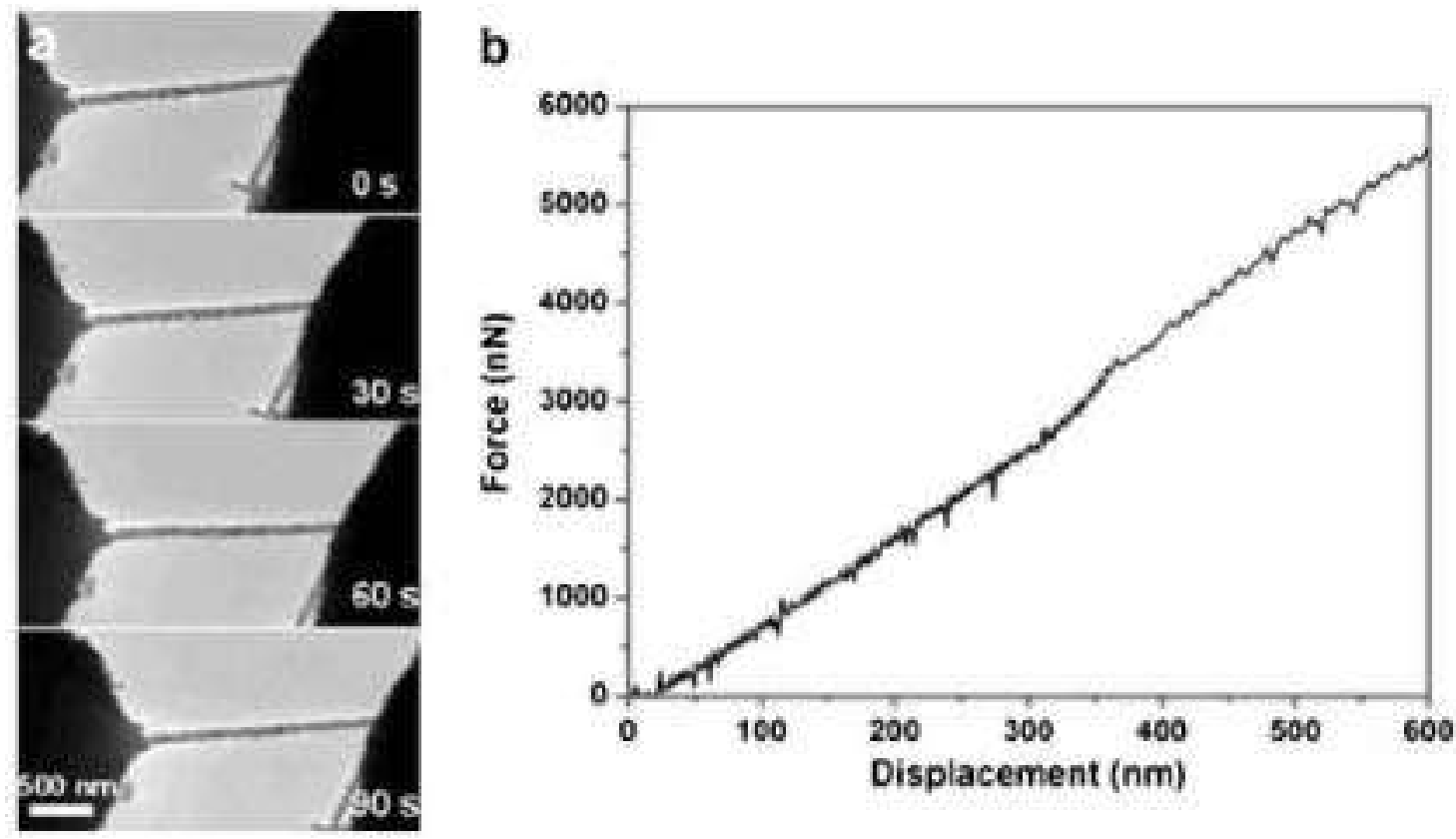

Figure 2.22. In-situ TEM tensile deformation of sputter-coated Al-BNNT nanohybrid and the corresponding force-displacement response ${ }^{15}$.

which far exceeds the typical ultimate tensile strength of $\mathrm{Al}$ (40 MPa). Further, the study states that there was no dislocation activity or $\mathrm{Al}$ crystal relocations during tensile deformation. The authors calculated the stresses developed in the Al coating and BNNT core using the elastic theory:

$$
\sigma_{A l, B N}=\frac{F \times E_{A l, B N}}{S_{A l} E_{A l}+S_{B N} E_{B N}}
$$

where $\mathrm{F}$ is the tensile force, $\mathrm{E}$ is elastic modulus and $\mathrm{S}$ is the cross-sectional area. The stresses were calculated to be $3.52 \mathrm{GPa}$ for $\mathrm{BNNT}$ core and $240 \mathrm{MPa}$ for $\mathrm{Al}$ coating. It is remarkable that the $\mathrm{Al}$ coating did not fail despite such high stresses. While the authors attribute the load-bearing ability to BNNT reinforcement, it is important to take into consideration the size effects in mechanical properties. The phenomenon "Smaller is 
Stronger" is widely observed for metals when the specimen dimensions are in submicrometer regime ${ }^{26-28}$. The authors' claim of nine times higher tensile stresses is based on a comparison with the ultimate tensile strength of bulk-sized Al. Comparing the mechanical response of these nano-sized composites with the macro-scale properties of $\mathrm{Al}$ may not represent the true reinforcement potential of BNNT.
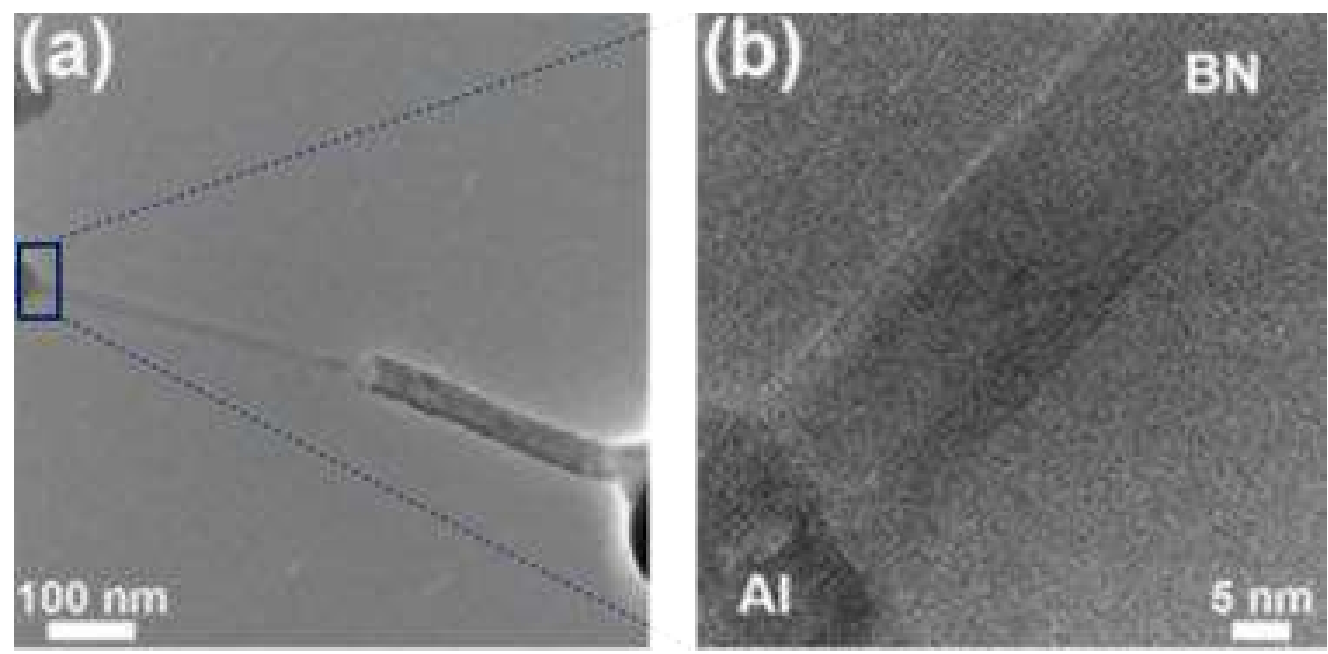

Figure 2.23. (a) Pull-out of inner BNNT shells during tensile loading of Al-BNNT, and (b) high magnification image of the Al-BNNT interface from where BN shells are pulled out ${ }^{15}$.

The study qualitatively touches upon the dependence of deformation mechanisms and load-bearing ability on the Al coating thickness. While a nanocomposite with $200 \mathrm{~nm}$ Al thickness did not fail under tension, a sample with just $20 \mathrm{~nm}$ Al thickness experienced brittle fracture (ultimate tensile strength $=1.47 \mathrm{GPa}$ ). It was reported that the inner shells of the multiwalled BNNT core slid out under tensile loading, while the outer shells were firmly bonded to Al (Figure 2.23). This observation suggests single-walled BNNT will provide superior reinforcement at higher stresses. Another possible strategy to overcome 
telescopic sliding is to fill the nanotube core with metal during synthesis, such that the inner walls are also bonded to the metal matrix. Clearly, in-situ observation of mechanisms is fruitful to come up with composite design strategies for tweaking the material properties.

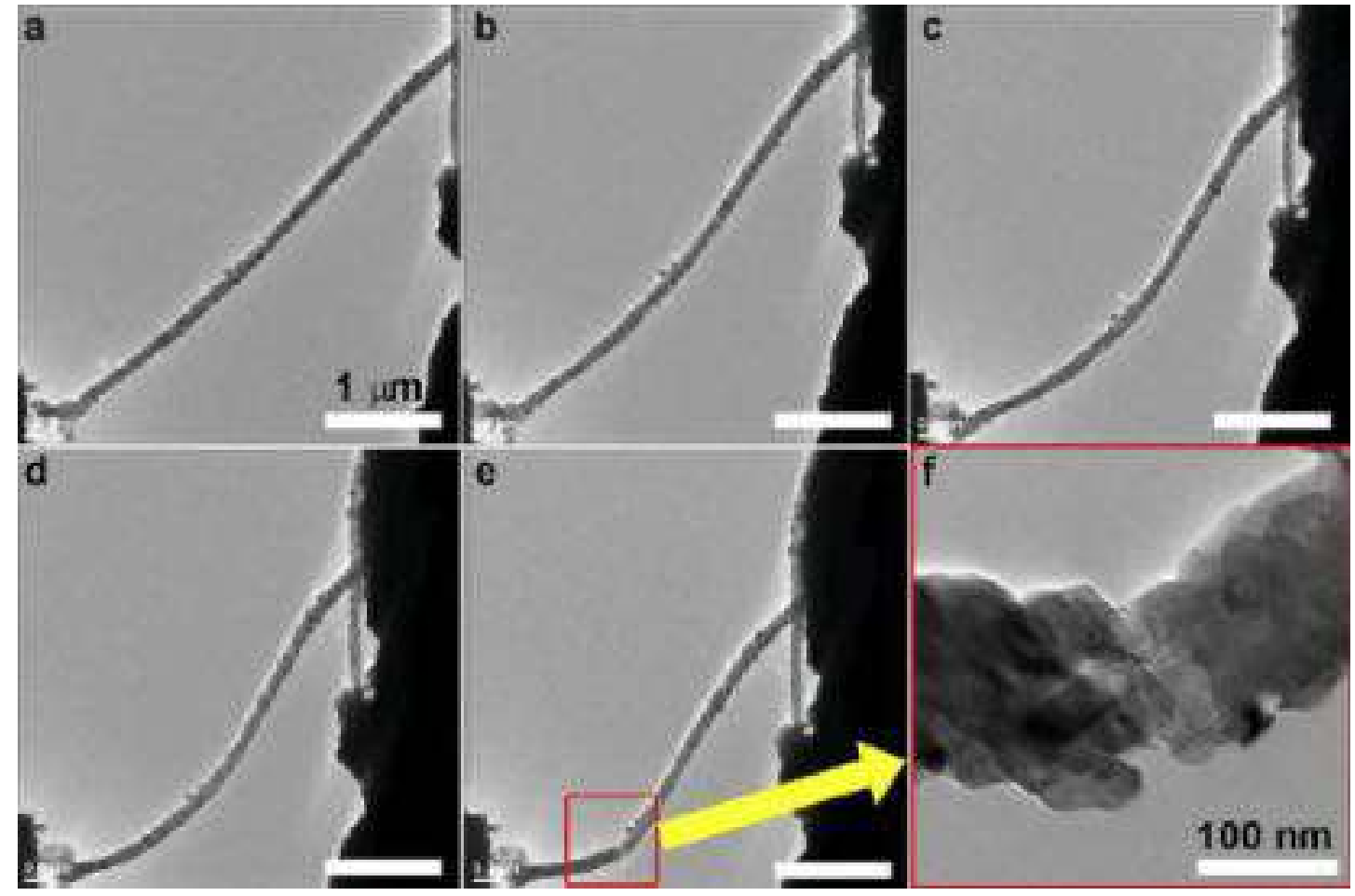

Figure 2.24. Real-time TEM snapshots of sputter-coated Al-BNNT nanocomposite undergoing bending deformation ${ }^{15}$.

\subsubsection{In-situ Bending and Fracture}

In-situ TEM bending of sputter-coated Al-BNNT nanohybrids is also reported in literature ${ }^{15}$. The composite displayed flexibility and failure-resistance under mechanical loading, as it transformed from a straight 1D morphology (before loading) to S-shaped configuration (Figure 2.24). The hybrid fractured as the bending angle reached about $\sim 50^{\circ}$. The authors reported the failure initiated in the outer Al deposit (in the tensed side). It was also noted that the crack propagation was not straightforward. Since the outer metal layer 
is composed of $\mathrm{Al}$ nanoparticles, the crack was deflected multiple times, dissipating mechanical energy. The authors suggested the crack propagation possibly followed the nanoparticle boundaries. As a result, the BNNT core did not fracture immediately and stayed intact for a while, resisting and delaying composite failure. As the strains further increased, partial pull-out of BNNT was observed. The study reported "sword-in-sheath" mechanism for nanotube core failure. These findings attest to the promise of BNNT for the toughening of MMCs.

\subsubsection{Critique}

The understanding of BNNT-MMC deformation is currently limited to nanoscale mechanisms. These studies examine the straining, fracture and pull-out mechanisms for metal-coated nanotube. The observed stress-transfer characteristics are highly informative for interface engineering strategies ${ }^{29}$. However, it is vital to interrogate BNNT-induced strengthening mechanisms in the microstructure at multiple length scales:

(i) Bulk-scale mechanisms in mm-cm sized samples are informative to correlate the stress-strain characteristics with composite density, grain sizes, nanotube orientation, and nanotube volume fraction ${ }^{30}$.

(ii) Meso-scale mechanisms ( $\mu \mathrm{m}$-mm length scales) are necessary to capture the interactions between multiple grains and the effect of nanotube distribution/ dispersion $^{31}$. 
(iii) Nano-micro-scale mechanisms $(\mathrm{nm}-\mu \mathrm{m})$ are essential to probe the response at grain boundaries, matrix-filler bonds and interfacial reaction products formed during composite synthesis ${ }^{32,33}$.

\subsection{Missing Gaps in Current Understanding: Some Critical Questions}

- What is the high-temperature endurance of long, high-aspect-ratio BNNT without any elemental impurities?

It was highlighted in Section 2.1 that the thickness of BNNT influences its oxidation resistance ${ }^{2}$. Does the length of nanotube also have a role to play? We believe longer BNNTs ( $l$ in the order of hundreds of $\mu \mathrm{m}$ ) should display superior oxidation resistance. This hypothesis is based on a prior observation that oxidation of BNNT typically initiates at the tube ends ${ }^{2}$. In the case of long nanotube clusters, the relative volume fraction of tube endings is much lower than the curved surface area of nanotubes, which should lead to arrested oxidation/ degradation. The hightemperature morphological transformations in long BNNTs are also unknown.

\section{- What are the high-temperature mechanical characteristics of BNNT?}

Enormous attention has been paid to the room temperature mechanical properties of BNNT, which have shown promise for engineering nanocomposites. However, there is no report on the load-bearing ability of BNNT at elevated temperatures. Without a thorough understanding of the temperature-mechanics correlation, it is impossible to determine if BNNTs have the potential for high temperature applications. 
- What is the effect of BNNT alignment on the load-bearing ability of MMCs? BNNTs display superior mechanical performance along their axial length. There is a lack of effort on engineering and mechanical assessment of directionally aligned BNNT-reinforced composites. Understanding orientation-dependence can provide critical cues for MMC microstructure design.

\section{- Can BNNTs survive in molten metal over a long duration of time?}

The answer to this question is important for engineering MMCs via the solidification (casting) route. Further, it is necessary to understand the wetting and chemical reactions at the molten metal-BNNT interface.

\section{- Can BNNTs be effectively integrated to form composites in ultra-short time}

\section{frames?}

The advent of additive manufacturing has captured the attention of researchers and industry alike because of the inherent advantage of fabricating near net-shaped structures. However, these processes are non-equilibrium in nature (ultra-fast cooling rates). Is it possible to achieve wetting and capture (internalization) of BNNT via the AM route?

\section{- Is BNNT suitable for low-ductility metals and alloys (Mg)?}

Mg has a ductility problem and secondary ceramic fillers often cause further deterioration ${ }^{34}$. While BNNT has demonstrated immense promise as a reinforcement material for $\mathrm{Al}$, is it feasible to translate the strengthening and toughening ability of BNNT to Mg without making it extremely brittle? There is no study on Mg-BNNT composite system. 
- What are the synergistic deformation mechanisms introduced because of BNNT addition and interfacial reactions? What are the critical stresses required to activate those mechanisms?

Site-specific in-situ mechanical investigations are required to develop a detailed understanding of deformation behavior. High-resolution real-time imaging vis-àvis force-displacement response will be critical to understand the load scale- and microstructure length-scale-dependence of deformation mechanisms ${ }^{35}$. Further, these investigations need to be performed as a function of processing parameters to discern the effect of interfacial reactions on the observed mechanisms.

The subsequent chapter seek to answer these critical questions which hold the key to develop BNNT-based lightweight and high-strength MMCs.

References

1. Golberg, D., Bando, Y., Kurashima, K. \& Sato, T. Synthesis and characterization of ropes made of BN multiwalled nanotubes. Scr. Mater. 44, 1561-1565 (2001).

2. Chen, Y., Zou, J., Campbell, S. J. \& Caer, G. Le. Boron nitride nanotubes: Pronounced resistance to oxidation. Appl. Phys. Lett. 84, 2430-2432 (2004).

3. Wei, X., Wang, M.-S., Bando, Y. \& Golberg, D. Tensile tests on individual multiwalled boron nitride nanotubes. Adv. Mater. 22, 4895-4899 (2010).

4. Yu, M. F., Yakobson, B. I. \& Ruoff, R. S. Controlled sliding and pullout of nested shells in individual multiwalled carbon nanotubes. J. Phys. Chem. B 104, 87648767 (2000). 
5. Tang, D.-M. et al. Mechanical properties of bamboo-like boron nitride nanotubes by in situ TEM and MD simulations: Strengthening effect of interlocked joint interfaces. ACS Nano 5, 7362-7368 (2011).

6. Huang, Y. et al. Thin boron nitride nanotubes with exceptionally high strength and toughness. Nanoscale 5, 4840-4846 (2013).

7. Zheng, M. et al. Radial mechanical properties of single-walled boron nitride nanotubes. Small 8, 116-121 (2012).

8. Yang, Y. H. \& Li, W. Z. Radial elasticity of single-walled carbon nanotube measured by atomic force microscopy. Appl. Phys. Lett. 98, 041901 (2011).

9. Chen, X., Dmuchowski, C. M., Park, C., Fay, C. C. \& Ke, C. Quantitative Characterization of Structural and Mechanical Properties of Boron Nitride Nanotubes in High Temperature Environments. Sci. Rep. 7, 11388 (2017).

10. Garel, J. et al. Ultrahigh torsional stiffness and strength of boron nitride nanotubes. Nano Lett. 12, 6347-6352 (2012).

11. Lahiri, D. et al. Boron nitride nanotubes reinforced aluminum composites prepared by spark plasma sintering: Microstructure, mechanical properties and deformation behavior. Mater. Sci. Eng. A 574, 149-156 (2013).

12. Yamaguchi, M., Meng, F., Firestein, K., Tsuchiya, K. \& Golberg, D. Powder metallurgy routes toward aluminum boron nitride nanotube composites, their morphologies, structures and mechanical properties. Mater. Sci. Eng. A 604, 9-17 (2014).

13. Singhal, S. K., Srivastava, A. K., Pasricha, R. \& Mathur, R. B. Fabrication of AlMatrix Composites Reinforced with Amino Functionalized Boron Nitride Nanotubes. J. Nanosci. Nanotechnol. 11, 5179-5186 (2011).

14. Yamaguchi, M. et al. Utilization of multiwalled boron nitride nanotubes for the reinforcement of lightweight aluminum ribbons. Nanoscale Res. Lett. 8, 3 (2013).

15. Yamaguchi, M., Tang, D., Zhi, C., Bando, Y. \& Shtansky, D. Synthesis, structural analysis and in situ transmission electron microscopy mechanical tests on individual aluminum matrix / boron nitride nanotube nanohybrids. Acta Mater. 60, 6213-6222 (2012).

16. Bhuiyan, M. M. H., Li, L. H., Wang, J., Hodgson, P. \& Chen, Y. Interfacial reactions between titanium and boron nitride nanotubes. Scr. Mater. 127, 108-112 (2017).

17. Obraztsova, E. A. et al. Metal ion implantation of multiwalled boron nitride nanotubes. Scr. Mater. 67, 507-510 (2012). 
18. Altieri, A. L. \& Steen, P. H. Adhesion Upon Solidification and Detachment in the Melt Spinning of Metals. Metall. Mater. Trans. B Process Metall. Mater. Process. Sci. 45, 2262-2268 (2014).

19. Bisht, A. et al. Effect of warm rolling and annealing on the mechanical properties of aluminum composite reinforced with boron nitride nanotubes. Mater. Sci. Eng. A 710, 366-373 (2018).

20. Edalati, K. \& Horita, Z. Application of high-pressure torsion for consolidation of ceramic powders. Scr. Mater. 63, 174-177 (2010).

21. Xue, Y. et al. Aluminum matrix composites reinforced with multi-walled boron nitride nanotubes fabricated by a high-pressure torsion technique. Mater. Des. 88, 451-460 (2015).

22. Bhuiyan, M. M. H. et al. Boron nitride nanotube reinforced titanium metal matrix composites with excellent high-temperature performance. J. Mater. Res. 32, 37443752 (2017).

23. Agarwal, A., Bakshi, S. R. \& Lahiri, D. Carbon Nanotubes: Reinforced Metal Matrix Composites. (CRC Press, Taylor \& Francis Group, 2011).

24. Baxter, S. C., Burrows, B. J. \& Fralick, B. S. Mechanical percolation in nanocomposites: Microstructure and micromechanics. Probabilistic Eng. Mech. 44, 35-42 (2016).

25. Wang, G., Yu, D., Mohan, R. V., Gbewonyo, S. \& Zhang, L. A comparative study of nanoscale glass filler reinforced epoxy composites: Electrospun nanofiber vs nanoparticle. Compos. Sci. Technol. 129, 19-29 (2016).

26. Uchic, M. D. \& Dimiduk, D. M. A methodology to investigate size scale effects in crystalline plasticity using uniaxial compression testing. Mater. Sci. Eng. A 400401, 268-278 (2005).

27. Greer, J. R., Th, J. \& Hosson, M. De. Plasticity in small-sized metallic systems : Intrinsic versus extrinsic size effect. Prog. Mater. Sci. 56, 654-724 (2011).

28. Kim, J. Y. \& Greer, J. R. Tensile and compressive behavior of gold and molybdenum single crystals at the nano-scale. Acta Mater. 57, 5245-5253 (2009).

29. Chu, K., Wang, J., Liu, Y. \& Geng, Z. Graphene defect engineering for optimizing the interface and mechanical properties of graphene/copper composites. Carbon $N$. Y. 140, 112-123 (2018).

30. Nautiyal, P., Embrey, L., Boesl, B. \& Agarwal, A. Multi-scale mechanics and electrical transport in a free-standing 3D architecture of graphene and carbon nanotubes fabricated by pressure assisted welding. Carbon N. Y. 122, 298-306 (2017). 
31. Boesl, B., Lahiri, D., Behdad, S. \& Agarwal, A. Direct observation of carbon nanotube induced strengthening in aluminum composite via in situ tensile tests. Carbon N. Y. 69, 79-85 (2014).

32. Yang, Y. et al. Unveil the Size-Dependent Mechanical Behaviors of Individual CNT/SiC Composite Nanofibers by In Situ Tensile Tests in SEM. Small 12, 44864491 (2016).

33. Zhou, W. et al. In-situ characterization of interfacial shear strength in multi-walled carbon nanotube reinforced aluminum matrix composites. Carbon N. Y. 106, 3747 (2016).

34. Trang, T. T. T. et al. Designing a magnesium alloy with high strength and high formability. Nat. Commun. 9, 2522 (2018).

35. Rudolf, C., Boesl, B. \& Agarwal, A. In Situ Mechanical Testing Techniques for Real-Time Materials Deformation Characterization. JOM 68, 136-142 (2016). 


\section{CHAPTER 3}

\section{THEORETICAL FRAMEWORK}

This chapter provides an overview of the relevant theories related to processing, microstructure design, and mechanics of nanotube-reinforced composites. The concepts of nanoparticle agglomeration, mechanical anisotropy, chemical tuning of stress-transfer, nanoparticle capture by solidification front, and complexity in understanding the mechanics of MMCs due to discrete-continuum duality are discussed at length. The theoretical framework presented here is connected with the research approaches and directions pursued in the dissertation. The goal of the chapter is to provide a rationale and blueprint of the investigations presented in detail in the subsequent chapters.

\subsection{Intertube Interactions}

\subsubsection{Nanoparticle Agglomeration}

One of the defining characteristics of nanoparticles is their extremely high surface area-tovolume ratio. As a result, they tend to aggregate together to reduce their free surface energy by making larger clusters ${ }^{1}$. Adhesion, capillary, and van der Waals forces hold together the nanoparticles as agglomerates. The strength of the agglomerates is given as ${ }^{2}$ :

$$
\frac{P}{A r}=\frac{f_{v}}{1-f_{v}} \frac{F}{d^{2}}
$$

where $P$ is the total binding force, $A r$ is the cross-section area of the agglomerate, $f_{v}$ is the volume fraction of the particle in the agglomerate, $F$ is the interparticle binding force and 
$d$ is the average diameter of the particle. For nanoparticle clusters, the size $(d)$ is extremely small $(<100 \mathrm{~nm})$. As a result, the binding forces in a cluster tend to be extremely high.

Eq. (3.1) can be modified for a BNNT cluster by replacing the $d^{2}$ term in the denominator with the product of nanotube diameter and length, $d^{*} l$, which is representative of the surface area of a cylinder $\left(A_{c y l}=\pi d l\right)$. Therefore, the strength of a BNNT cluster can be determined using the following relationship:

$$
\left(\frac{P}{A r}\right)_{B N N T} \approx \frac{f_{v}}{1-f_{v}} \frac{F}{d . l}
$$

A cluster comprises of entangled nanotubes and inter-tube spacing (or porosity). Therefore, the actual volume fraction of solid nanotubes, $f_{v}$, is equal to ( 1 - porosity). The starting BNNT samples used in this dissertation were generally characterized by $\sim 80 \%$ porosity (or $20 \%$ BNNT packing density). Therefore, the value of $f_{v}$ is $\sim 0.2$. The strength of BNNT agglomerates can be determined if the binding force between the nanotubes is known. Tang and co-workers determined the van der Waals attraction force between two overlapping BNNTs to be $\sim 96 \mathrm{nN}$ by in-situ TEM investigation ${ }^{3}$. This force measurement was performed for a nanotube with $27.5 \mathrm{~nm}$ diameter $(d)$ and an overlapping length $(l)$ $87 \mathrm{~nm}$. Substituting these values to Eq. (3.2), the strength of a BNNT cluster is found to be $\sim 10 \mathrm{MPa}$.

To put this into perspective, the above-calculated strength of a BNNT cluster is comparable to the yield strength of wood or several elastomers (Figure 3.1). The absolute values will vary depending on nanotube morphologies, dimensions, structural defects, and 
impurities. Nevertheless, this estimation gives a good idea of the extent of inter-tube interactions in BNNT.

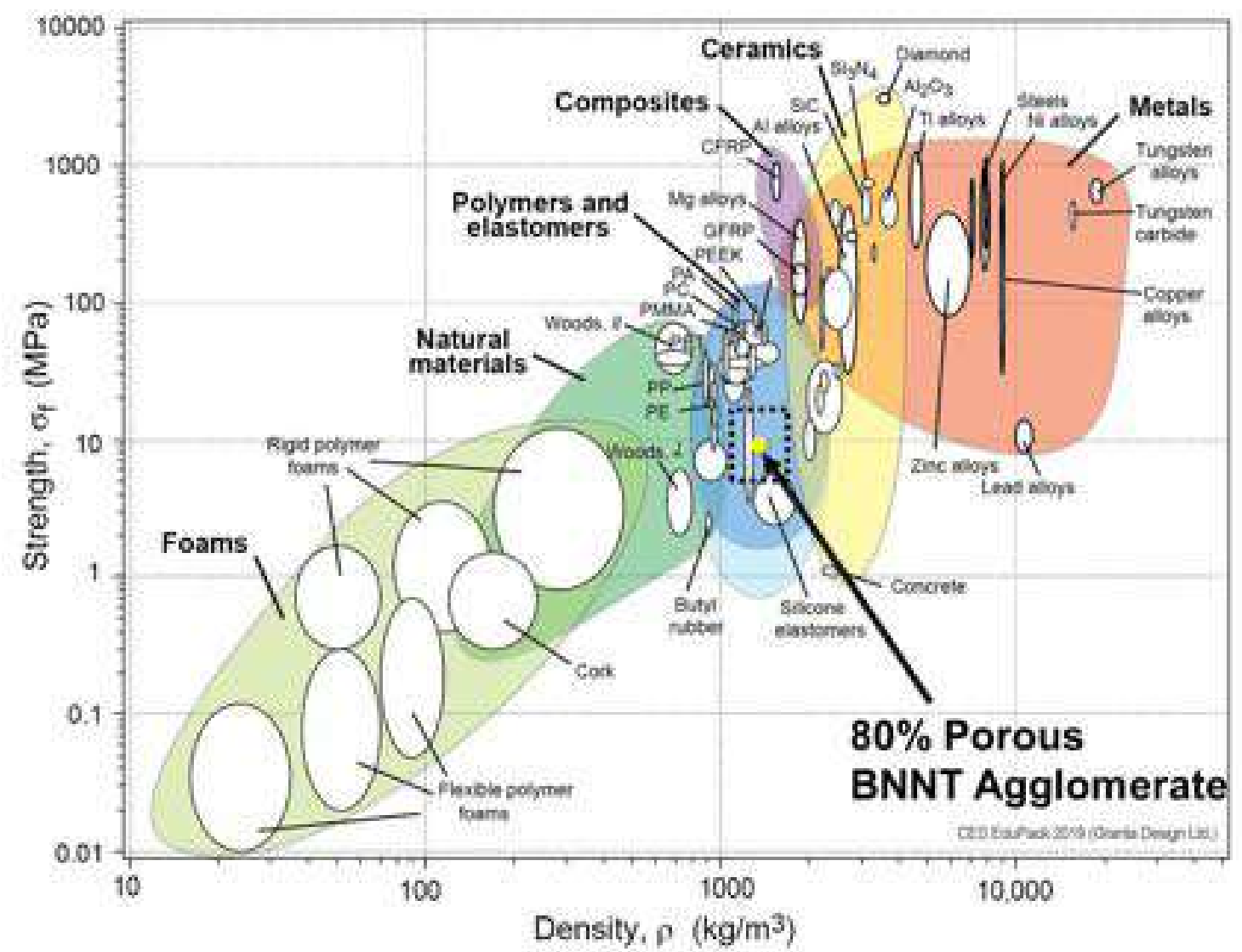

Figure 3.1. The relative position of a (20\% dense) BNNT agglomerate in the Ashby map, showing reasonably strong inter-tube bonding in the clusters.

Adapted from Granta Design https://grantadesign.com/education/students/charts/

\subsubsection{Implication for Metal Matrix Composite Manufacturing}

The calculations based on the modified cluster strength formula highlights a strong tendency of BNNTs to agglomerate. Clusters can be detrimental because of a few reasons:

(i) It can be challenging to infuse or infiltrate metal inside the inter-tube crevices or pores in the clusters. The inter-tube separations are extremely small (generally $<1$ 
$\mu \mathrm{m})$. Micron-sized metal particles or viscous melt may not be able to fill these gaps during processing, resulting in dominant porosities in the MMC microstructure ${ }^{4}$.

(ii) Nanotube clusters act as potential sites for stress-concentration during mechanical loading. This results in premature failure initiation, compromising the mechanical properties of the composite.

(iii) The local concentration of BNNT will lead to inhomogeneous mechanical properties. BNNT reinforcement effect will not be felt uniformly, which can cause the material to behave/ deform in a very unpredictable fashion.

These challenges call for a plan of action to break and distribute the nanotubes throughout the metal matrix to effectively exploit the superior mechanical properties of BNNT.

\subsubsection{Approach Adopted: Physical-Chemical Dispersion to Address Clustering}

An additional step of BNNT dispersion is necessary before MMC processing. Liquid solvents serve as a convenient system to add, de-agglomerate and disperse nanotubes. Based on the theoretical calculation above, a key component of the BNNT dispersion strategy will be to impart sufficient energy to the dispersion medium. Unlike conventional nanoparticles, BNNTs can entangle or intertwine with each other owing to their high aspect ratio. The dispersion process will involve a disentanglement step, followed by a separation step. Therefore, it may be necessary to subject the solvent-BNNT suspension to mechanical energy for an extended duration of time for effective dispersion. 
The second important consideration is ensuring the dispersed BNNTs do not reaggregate after the external source of mechanical energy is removed. It was discussed before that the lowering of free surface energy is the driving force for nanomaterial agglomeration. A possible approach to prevent re-agglomeration is by stabilizing the suspension of BNNT in the solvent ${ }^{5}$. In this work, we accomplished this by BNNT surface modification to lower its surface tension with the solvent ${ }^{6}$. Consequently, the necessity for surface energy minimization is no longer felt and making the BNNT-solvent dispersion stable even after the external source of energy is removed. Surface modification, when coupled with mechanical/ physical dispersion, will be able to reduce the total time required for de-agglomeration. Shorter dispersion time will ensure that the BNNTs are not damaged/ fragmented due to excessive mechanical energy ${ }^{7}$.

\subsection{Nanotube Orientation vis-à-vis Mechanical Properties of Composite}

\subsubsection{Orientation-Sensitive Shear Lag Model}

In Chapter 2, the shear lag model was briefly discussed to highlight the concept of critical length for composite strengthening. Owing to their 1D morphology, BNNT has anisotropic properties and displays superior load-bearing ability along the axial direction (length of a nanotube). Therefore, it's prudent to utilize a modified shear lag model which can predict the yield strength of MMC based on fiber orientation ${ }^{8}$ :

$$
\sigma_{y}=\frac{V_{f} \sigma_{m}}{2} S_{e f f}+\sigma_{m}
$$


where $\sigma_{f}$ is the fiber strength, $\sigma_{m}$ is the matrix strength and $S_{\text {eff }}$ is the effective aspect ratio of an elongated nanotube cluster oriented at an angle $\theta$ to the loading direction (Figure 3.2). The average value of $S_{\text {eff }}$ is obtained as:

$$
S_{e f f, A v}=\int_{0}^{\pi / 2} S_{e f f}(\theta) F(\theta)(2 \pi \sin \theta) d \theta
$$

where $F(\theta)$ is the probability distribution function of the misorientation of the BNNT/metal clusters.

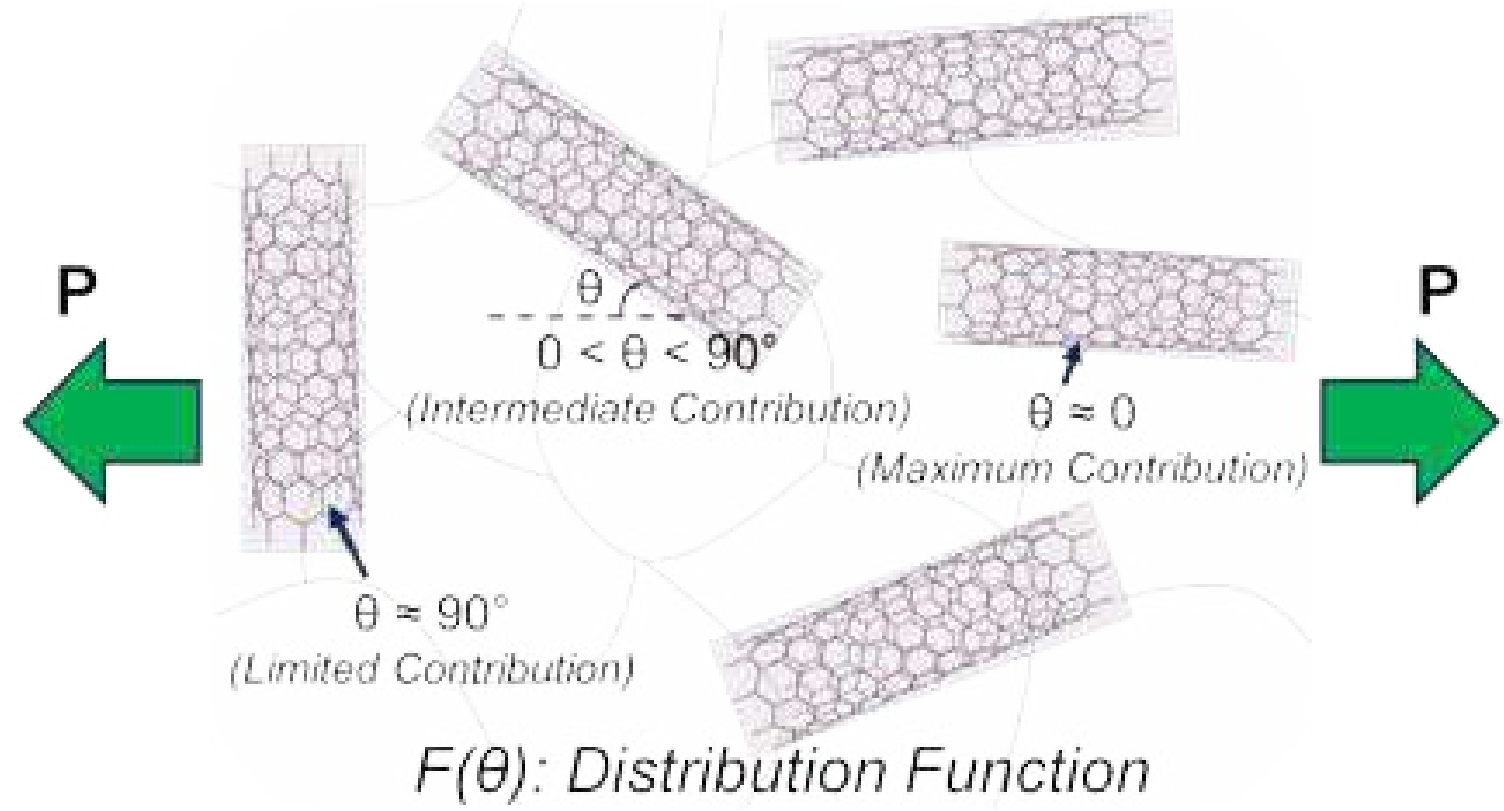

Figure 3.2. A schematic representation highlighting the importance of nanotube orientation in MMC microstructure for load-bearing ability owing to BNNT's high aspect ratio.

\subsubsection{Application to this Work: Engineering Percolated Composite Microstructure}

The concept of geometric percolation was highlighted in the previous chapter. Achieving an interconnected network of nanofillers by adding sufficiently high filler fraction is 
desired for impactful reinforcement. A combination of geometric percolation and desired orientation should produce remarkable mechanical properties. The high volume fraction of nanotubes can lead to the formation of local composite clusters in the MMC. The composite clusters are the nanotube-rich regions in the microstructure, which are well-infiltrated/ wetted by the $\operatorname{metal}^{9}$. These composite clusters should not be confused with the porous agglomerates mentioned in Section 3.1. Instead, the composite clusters are dense and porefree, with brilliant local mechanical strength. It has been reported that the formation of local composite clusters leads to a second yield point during mechanical testing, in addition to the one mentioned in Eq. (3.3). This secondary yield point is modeled using the rule of mixture:

$$
\sigma_{y, 2}=\sigma_{y, 1}\left(1-V_{f}\right)+\sigma_{f} V_{f}
$$

Multi-stage yielding delays the failure of composite and dissipates mechanical work. This model allows us to estimate the mechanical performance based on BNNT volume fraction and orientation. The current research utilizes this theoretical framework (Eq. $3.3-3.5$ ) to simultaneously exploit the orientation and percolation effects for designing MMC microstructure with extraordinary performance.

\subsection{Nanocomposite Interface Mechanics}

\subsubsection{Matrix-Filler Interphases}

Due to high-temperature conditions involved in MMC processing, BNNT and metal can react to form new phases at the interface. Interface reactions result in covalent bonding 
between the matrix and the filler, which can be desirable for effective stress-transfer during mechanical loading. The covalently bonded nanotubes are difficult to detach and pull-out of the composite due to external loading. The strength of MMC with interphase(s) is modeled as ${ }^{10}$ :

$$
\sigma_{c}=\left(1+\frac{2 b}{D}\right)\left[\frac{\sigma_{\text {shear }} l}{D}-\left(1+\frac{2 b}{D}\right) \sigma_{m}\right] V_{f}+\sigma_{m}
$$

where $\sigma_{\text {shear }}$ is the shear strength of the interphase, $b$ is the width of the interphase and $D$ is nanotube diameter. It is clear from this equation that the strength of the composite is dependent on the shear strength of the interphases. It should be noted that the interfacial reactions produce ultra-fine crystals or layers of new phases. The "Smaller is Stronger" phenomenon is expected in these nanosized products, meaning the strength of the interphases should be much higher than their reported bulk/ macro-scale mechanical properties $^{11-13}$. Hence, the prediction of MMC strength by feeding in macroscale properties may lead to an underestimated value. On the other hand, non-uniform reactions and formation of phases with significant flaws can be detrimental to mechanical performance, and the actual properties may be lower than the predicted properties.

\subsubsection{Application to this Work: Chemical Tuning of Interfacial Stress-Transfer}

Thermochemical calculations have indicated the feasibility of chemical reactions between $\mathrm{Al}$ and BNNT (h-BN phase) at elevated temperatures. For instance, analysis at $650^{\circ} \mathrm{C}$ revealed a negative change in Gibbs free energy $(\Delta \mathrm{G}=-13.9 \mathrm{~kJ})$ for the formation of AlN and $\mathrm{AlB}_{2}{ }^{14}$ : 

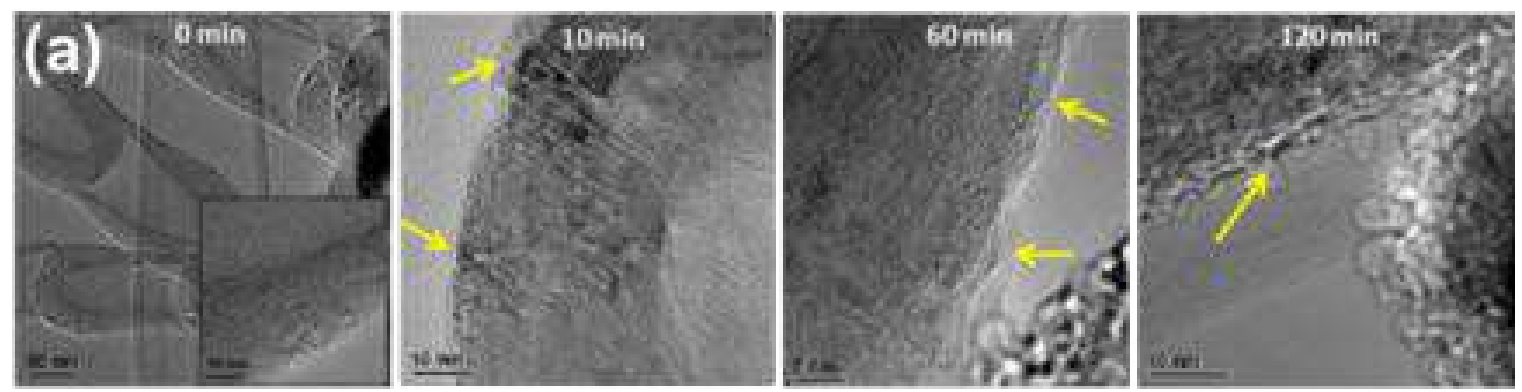

\section{Increasing Thermal Exposure Time}

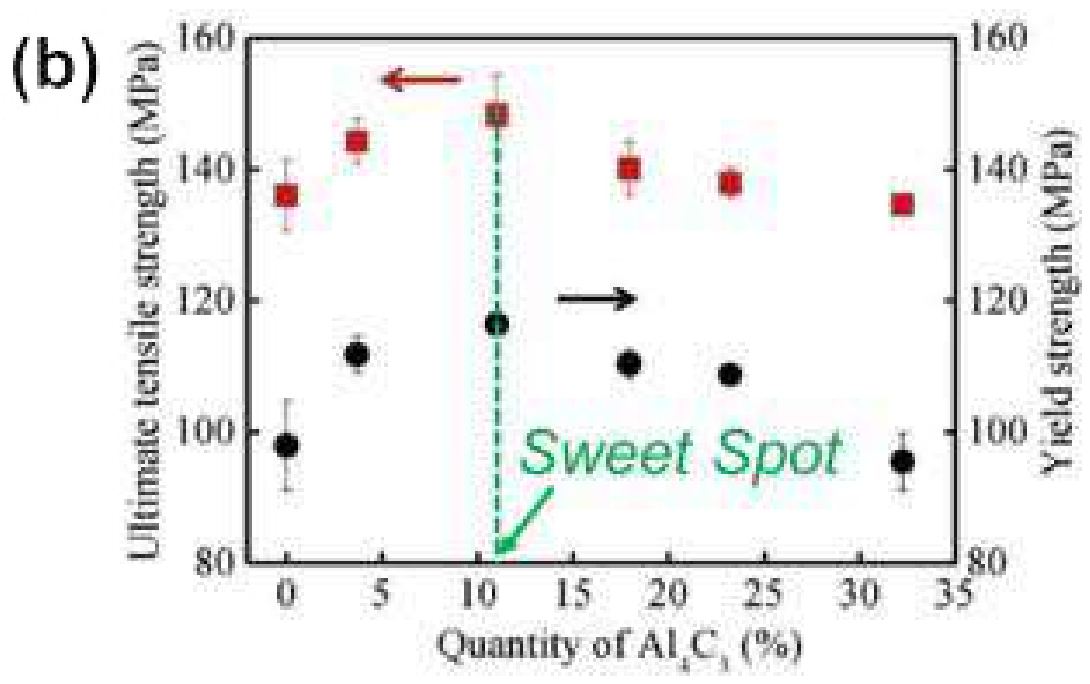

Figure 3.3. (a) Kinetics of Al-BNNT interfacial reactions (reaction products as a function of thermal exposure time) ${ }^{14}$, and (b) tensile strength and yield strength of Al-CNT as a function of interfacial reaction product fraction ${ }^{15}$.

$$
(95.77) A l+(4.23) B N=(88.72) A l+(6.98) A l N+(4.14) A l B_{2}
$$

Furthermore, thermochemical analysis of the reaction of $\mathrm{Al}$ with $\mathrm{B}$ and $\mathrm{N}$ individually indicated a preference for the formation of $\mathrm{AlN}$ over $\mathrm{AlB}_{2}$ at similar temperature conditions. $\Delta \mathrm{G}$ was two orders of magnitude smaller for AlN formation ($105.2 \mathrm{~kJ})$ as compared to $\mathrm{AlB}_{2}$ formation $(-5.1 \mathrm{~kJ})^{14}$. Lahiri et al. investigated the reaction kinetics at the Al/BNNT interface by HRTEM analysis as a function of high-temperature 
exposure time (Figure 3.3a) ${ }^{14}$. It was observed that as the exposure time increased from 1 $\mathrm{h}$ to $2 \mathrm{~h}$, the average interfacial product thickness increased from $1.5 \mathrm{~nm}$ to $7.3 \mathrm{~nm}$. Therefore, processing time (in addition to the temperature) is also a critical factor for tuning the interface chemistry in BNNT-MMCs. The extent of new phase formation is important from the standpoint of mechanical properties; while limited reaction is helpful for intimate bonding, excess reactions can degrade BNNT and compromise its intrinsic properties. There is recorded evidence for CNT-Al composites that there is indeed a "sweet spot" for the extent of acceptable reactions, after which the mechanical properties start deteriorating (Figure 3.3b $)^{15}$. The manufacturing technique and processing conditions will be selected to strike a fine balance between reactions and preservation.

\subsection{Solidification Front-Nanotube Interactions}

\subsubsection{Nanoparticle Capture-Repulsion Dichotomy}

Capturing BNNT in the melt is necessary for manufacturing MMCs with uniform distribution of filler phase by solidification routes, such as casting. However, capturing nanomaterials (particularly with size less than $100 \mathrm{~nm}$ ) is challenging because of repulsive interactions, such as van der Waals force, intermolecular interactions, interfacial force, and the integrated disjoining pressure ${ }^{16}$. These forces push away the nanoparticles in front of a moving solidification front, resulting in an energy barrier (Figure 3.4a). The van der Waals potential between a nanoparticle and a solid plane is expressed by the relation ${ }^{17}$ :

$$
W_{v d w}(D)=-\frac{A R}{6 D}
$$


where $D$ is the metal-ceramic separation distance, $R$ is the nanoparticle radius and $A$ is the Hamaker constant. For a nanomaterial capture system, the Hamaker constant is described by the relationship ${ }^{16}$ :

$$
A_{\text {sys }} \approx\left(\sqrt{A_{\text {solid }}}-\sqrt{A_{\text {liquid }}}\right)\left(\sqrt{A_{\text {nanomaterial }}}-\sqrt{A_{\text {liquid }}}\right)
$$

The repulsive van der Waals forces exerted on the nanoparticles can be obtained by the derivative of this potential expression ${ }^{16}$ :

$$
F_{v d w}(D)=-\frac{\partial W_{v d w}}{\partial D}=-\frac{A_{s y s} R}{6 D^{2}}
$$

On the other hand, a viscous drag force opposes the repulsion of nanoparticles. This force can be derived from the Stokes' law after incorporating suitable modifications accounting for nano-scale interactions associated with a nanoparticle moving in a melt ${ }^{16,18,19}$ :

$$
F_{v i s}(D)=6 \pi \frac{D}{D-2 D_{s}} \eta_{b u l k} v \frac{R^{2}}{D}
$$

where $D_{S} \sim$ diameter of the liquid molecule, $\eta_{b u l k}$ is the viscosity of the bulk liquid and $v$ is the velocity of the moving nanoparticle.

Therefore, the net total of the attractive (viscous drag) and repulsive (van der Waals) forces acting on a nanoparticle can be expressed by the addition of Eq. 3.10 and 3.11. The drag force slows down the nanoparticles pushed away by van der Waals forces; this allows the solidification front to quickly catch up with the nanofillers (capture phenomenon). Xu et al. determined the critical velocity expression for the capture of nanoparticles from the total force expression ${ }^{16}$ : 

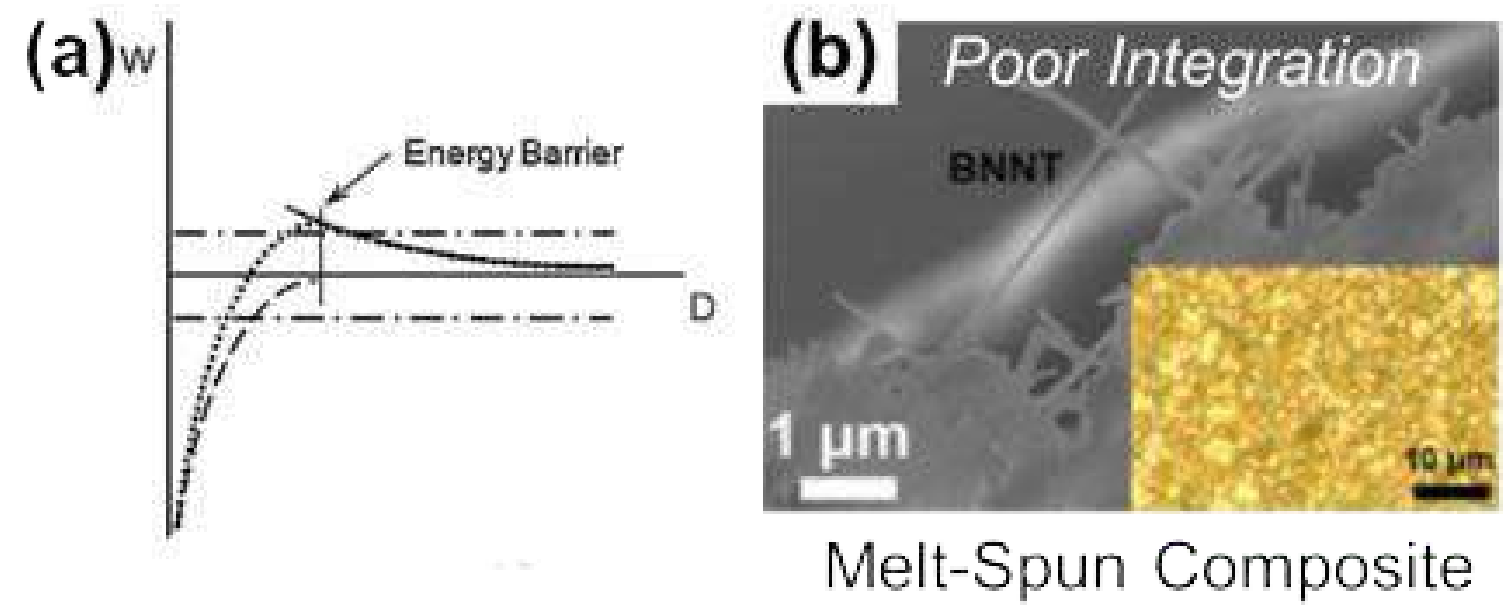

Figure 3.4. (a) Illustration of an energy barrier for nanomaterial capture in molten metal due to repulsive forces ${ }^{16}$, and (b) SEM micrograph of a melt-spun Al-BNNT composite with poor integration highlighting the importance of meeting capture criterion ${ }^{20}$.

$$
v_{c r}=\frac{A_{s y s}\left(D_{c r}-2 D_{s}\right)}{36 \pi R \eta_{b u l k} D_{c r}^{2}}
$$

where $D_{c r}$ is the critical distance between the solidification front and the nanoparticles for the capture of nanoparticles. This relationship predicts high critical velocities for capturing fine nanoparticles. For instance, the calculated critical velocities for capturing $\mathrm{TiB}_{2}$ and $\mathrm{Al}_{2} \mathrm{O}_{3}$ in $\mathrm{Al}$ melt are 220 and $3200 \mu \mathrm{m} / \mathrm{s}$, respectively ${ }^{16}$. These values are orders of magnitude higher than the typical solidification front velocity in mold casting $(\sim 1 \mu \mathrm{m} / \mathrm{s})$. This poses a challenge for the effective integration of nanotubes via solidification routes. The problem was evident from the micrographs in a prior study on melt-spun Al-BNNT, the only report on BNNT-MMC synthesis by solidification technique (Figure 3.4b). The SEM image shows poor wetting and integration of BNNTs. 

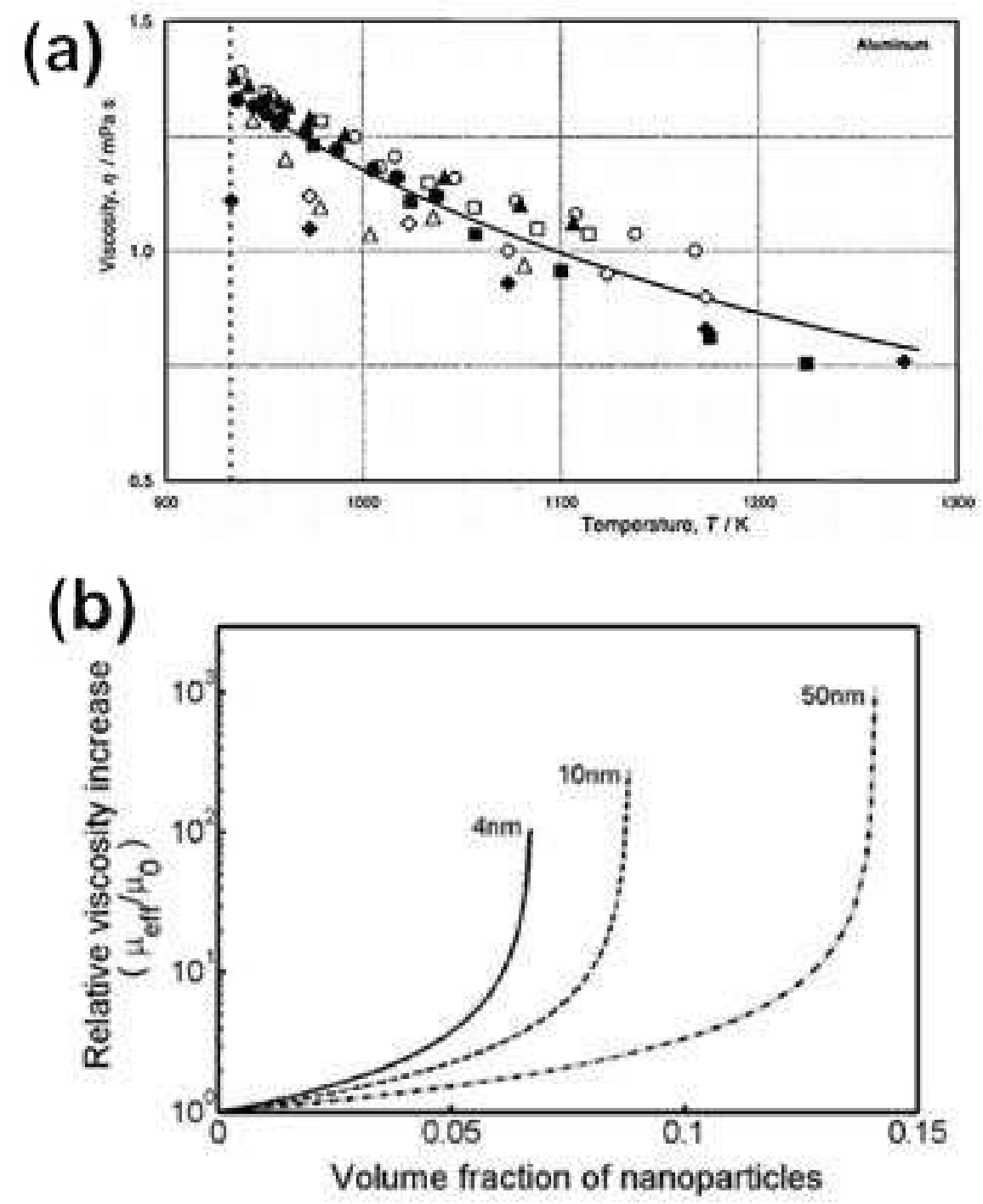

Figure 3.5. (a) Effect of temperature ${ }^{21}$ and (b) effect of nanofiller volume fraction ${ }^{16}$ on the viscosity of $\mathrm{Al}$ melt.

\subsubsection{Application to this Work: Strategies for Solidification Processing}

Approach A: Efforts to bring down the critical velocity requirement

The ultra-high critical speed can be lowered to some extent by increasing the viscosity of the melt, $\eta_{\text {bulk }}$ (based on Eq. 3.12). There are a couple of ways to achieve: 
(i) Limiting overheat in the melt: Viscosity of molten Al is highly temperaturesensitive. Regression analysis by Assael et al. captures this effect ${ }^{21}$ :

$$
\log _{10}\left(\eta / \eta^{0}\right)=-a_{1}+\frac{a_{2}}{T}
$$

where $\eta_{0}=1 \mathrm{MPa}$ s, $a_{1}=0.7324$ and $a_{2}=803.49 \mathrm{~K}$. Increasing the melt temperature from $660^{\circ} \mathrm{C}$ to $1000^{\circ} \mathrm{C}$ causes the viscosity to drop by an order of magnitude (Figure 3.5a). Therefore, choosing relatively lower temperatures (near melting point) during solidification processing will be helpful for capturing BNNTs in the melt.

(ii) High volume fraction of BNNT: Adding solid nanofillers in molten metal results in enhanced viscosity. The relative increase in viscosity due to nanofiller is expressed by the empirical relationship ${ }^{22}$ :

$$
\frac{\mu_{e f f}}{\mu_{0}}=\frac{1}{1-34.87\left(d_{p} / d_{f}\right)^{-0.3} \varphi^{1.03}}
$$

where $d_{p}$ and $d_{f}$ represent the diameters of nanoparticle and liquid molecule. Theoretical calculations performed by $\mathrm{Xu}$ et al. reported up to 2 orders of magnitude enhancement in viscosity by adding 5-10 vol.\% of nanoparticles $(\text { Figure 3.5b })^{16}$.

$\underline{\text { Approach B: Alternative solidification strategies }}$

Additive manufacturing (AM) techniques are promising to fulfill the enhanced critical velocity requirement for capturing fine nanotubes. Metal AM techniques are known for ultrahigh cooling rates. In many circumstances, this non-equilibrium solidification has 
been viewed unfavorably because of hierarchical and irregular microstructures and anisotropic mechanical properties. However, the orders of magnitude higher solidification front velocity afforded by AM techniques provides a fantastic opportunity to capture ultrafine nanotubes in molten metals. Moreover, AM techniques offer rapid material deposition/ fabrication ability, which is desirable for scalable manufacturing. Therefore, exploring the development of BNNT-MMCs via AM route holds promise to overcome the repulsion-attraction dichotomy outlined above.

\subsection{Strength-Ductility Trade-off}

\subsubsection{Dislocation Obstruction}

Nanotubes often obstruct dislocation motion in the metal matrix, creating high dislocation density in the microstructure (Figure 3.6a). This can result in Orowan looping mechanism, creating a back-stress and enhancing the shear strength of the composite:

$$
\Delta \tau=\frac{A}{r \ln \left(2 r / r_{0}\right)} G b V_{f}^{1 / 2}
$$

where $r_{0}$ is the radius of the dislocation core, $r$ is the volume equivalent radius for nanotube, $G$ is the shear modulus, $b$ is the Burgers vector of the dislocations, $A$ is a dislocationdependent constant $\left(A=0.093\right.$ for edge dislocation and 0.14 for screw dislocation), and $V_{f}$ is the volume fraction of nanotubes in the composite. However, the repulsion of dislocation motion at nanotube-rich sites leads to arrested plasticity, significantly lowering the strainto-failure of the composites. This poses an impediment for reinforcing matrices with poor ductility, such as lightweight magnesium alloys. Deterioration in plasticity leads to arrested 
formability, making it challenging to fabricate sheets and wires out of the composite. Further, low ductility can lead to catastrophic failure of structural components, limiting the widespread application of the composites.
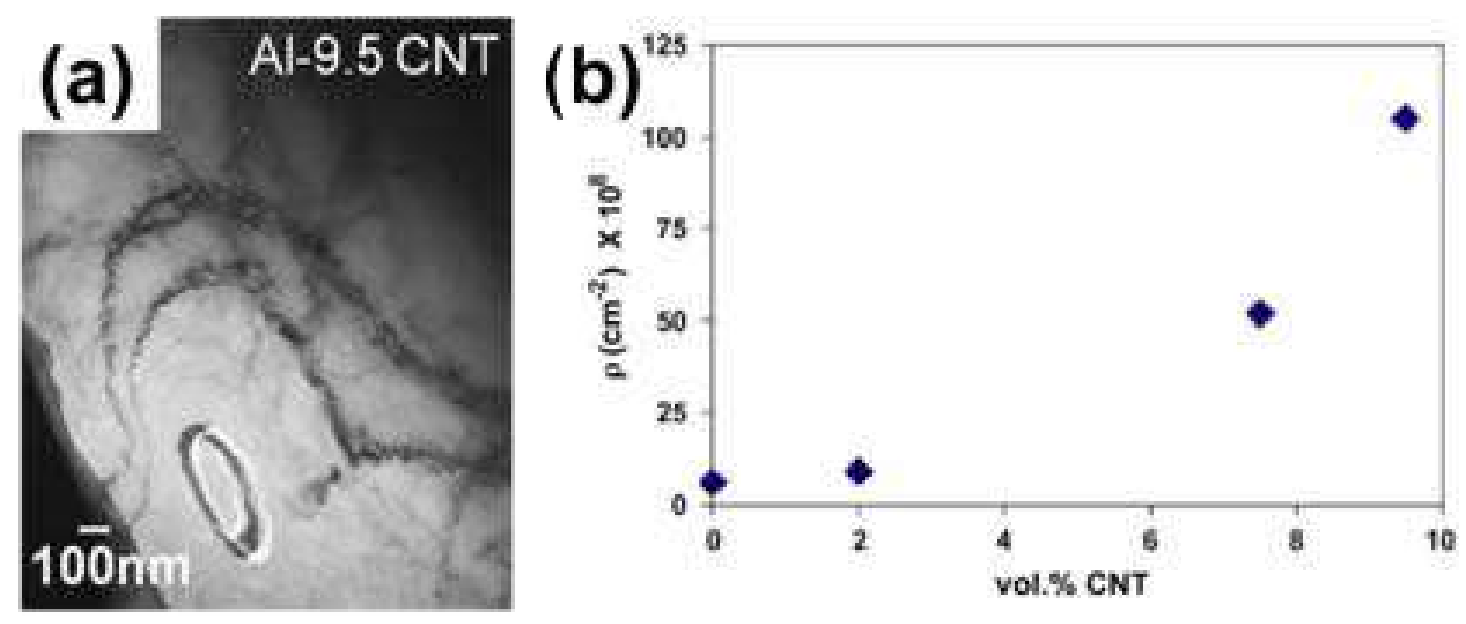

Figure 3.6. (a) TEM micrograph of a CNT-Al composite exhibiting dislocation loop formation, and (b) computed dislocation density as a function of nanotube content demonstrating dislocation pileups in the microstructure because of 1D nanofillers ${ }^{24}$.

\subsubsection{Approach Adopted in this Work: Architected Nanocomposites}

As stated above, dislocation motion generally drives plasticity in metals. Continuous distribution of BNNTs throughout the matrix produces extensive hindrance to dislocations.

One possible solution is to add a relatively lower volume fraction of BNNT to limit the dislocation density (Figure 3.6b). However, creating a percolated microstructure may not be possible with low filler content, as discussed in Chapter $2^{23}$. Engineering composite microstructure to have specific zones for uninterrupted dislocation motion can be an effective strategy to overcome plasticity loss. For instance, a multi-layer architecture with alternating metal-rich and nanotube-rich zones can be designed, which will contribute 
towards ductility and strengthening, respectively. A sandwich composite (metal/BNNT/metal) is an example of such a composite structure. If the interfaces between the zones are strong, two-way stress-/ strain-transfer is expected: BNNT-rich regions will provide the ability to sustain higher loads, and the metallic zones will experience plastic mechanisms. BNNT can facilitate crack deflection and crack-bridging, owing to its high aspect ratio. The crack arrest behavior should further enhance the ductility by delaying the failure point. The integrity of interface is critical to realize these mechanisms, as poor bonding can lead to premature delamination, sliding and pull-out. The strength-ductility trade-off will still be present, but this approach can be promising for working with low ductility metals.

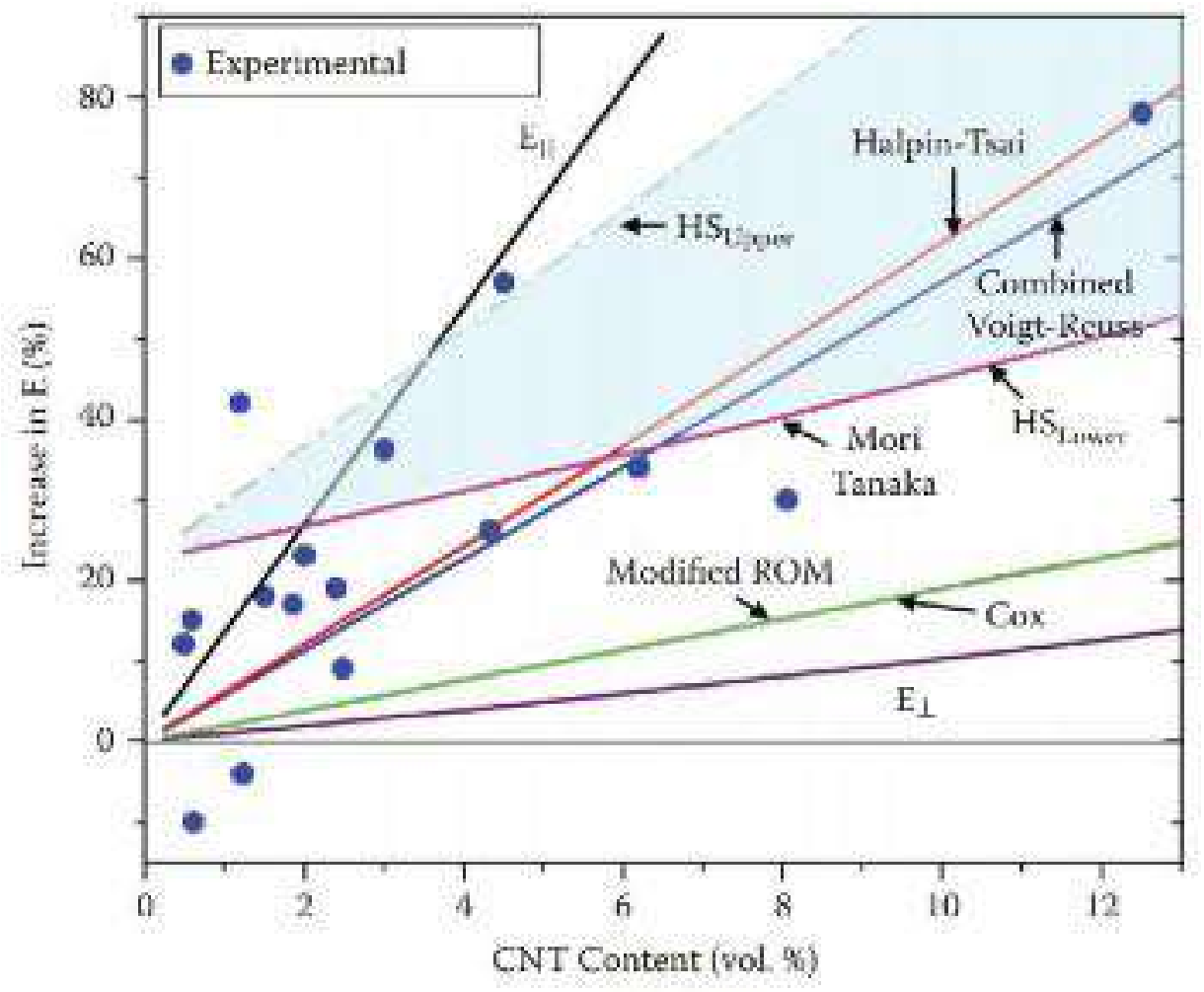

Figure 3.7. Comparison of micromechanics models and experimental data points for CNT-MMC, highlighting the difficulty of predicting the elastic modulus?. 


\subsection{Discrete-Continuum Duality}

\subsubsection{Hierarchical Microstructure, Interface Complexity and Size-Effect}

Continuum mechanics framework, at times, fails to explain the mechanical response of nanocomposites owing to their inherent hierarchical structure ${ }^{25}$. The micromechanical modeling strategies do not take into consideration all the complex interfacial interactions (chemical, physical and/or a combination of both). Unique microstructure features and deformation mechanisms, such as dispersion/ clustering $^{4}$, multiple reaction products ${ }^{14,26}$, capillarity-induced filling of nanotube by metal ${ }^{27,28}$, interface sliding and telescopic sliding $^{29}$, to list a few, makes the understanding of mechanical response challenging. Moreover, with the advent of metal additive manufacturing, components with characteristic feature sizes in sub-millimeter length scales are being fabricated. In MMCs, the nanotube reinforcement structure (e.g. isolated tubes, ropes, yarns or mats) can be several hundreds of micrometer in length. As the characteristic feature size becomes comparable to the constituent microstructure, failure analysis using conventional models, such as beam theory, may not be applicable ${ }^{30}$. This is because the underlying assumption or treatment of nanotube-reinforced composites as a continuous structure is not valid at small length scales. Figure 3.7 demonstrates the deviation of experimental results from the micromechanics models; this plot shows the enhancement in elastic modulus of metal matrices due to CNT addition. The experimental data points follow some of the models (with mixed accuracy), where the assumptions of the continuum mechanics approach hold true. However, the breakdown of the micromechanics models observed for several data points is indicative of the departure from the continuum regime. The development of novel 
BNNT-based composites necessitates systematic scrutiny of the discrete-continuum duality phenomenon.

\subsubsection{Approach Adopted in this Work: Multi-scale and In-situ Mechanical Investigation}

The mechanical properties of nanocomposites are site-dependent as well as length-scale dependent. Examining the mechanical response at multiple load and length scales, and under different stress-states can unravel the stress-transfer behavior in BNNT-MMC microstructures (Figure 3.8). Targeted loading in the nano- to micrometer length scale can help in understanding the local reinforcement effect of BNNT. The mesoscale characterization will provide an understanding of the interactions between multiple constituents, such as nanotubes, interphases, grains, precipitates, and other defects. A macro-scale test provides an estimate of the overall mechanical response, where contributions of all the material features are accounted for. It is noteworthy that several features of interest in the MMC microstructure are extremely small, and it's not possible to probe their response using conventional measurement techniques. Hence, coupling mechanical tests with high-resolution imaging (e.g. electron microscopes, atomic force microscope) is essential to resolve and target those features of interest. In-situ mechanical testing facilitates direct observation of deformation mechanisms and nanotube-induced strengthening. Simultaneously captured force-displacement response is useful to quantify critical stresses/ strains for the activation of different mechanisms in the composite. 
Obtaining these mechanistic insights is critical to identify suitable processing conditions and develop microstructure design strategies for achieving superior properties in MMCs.

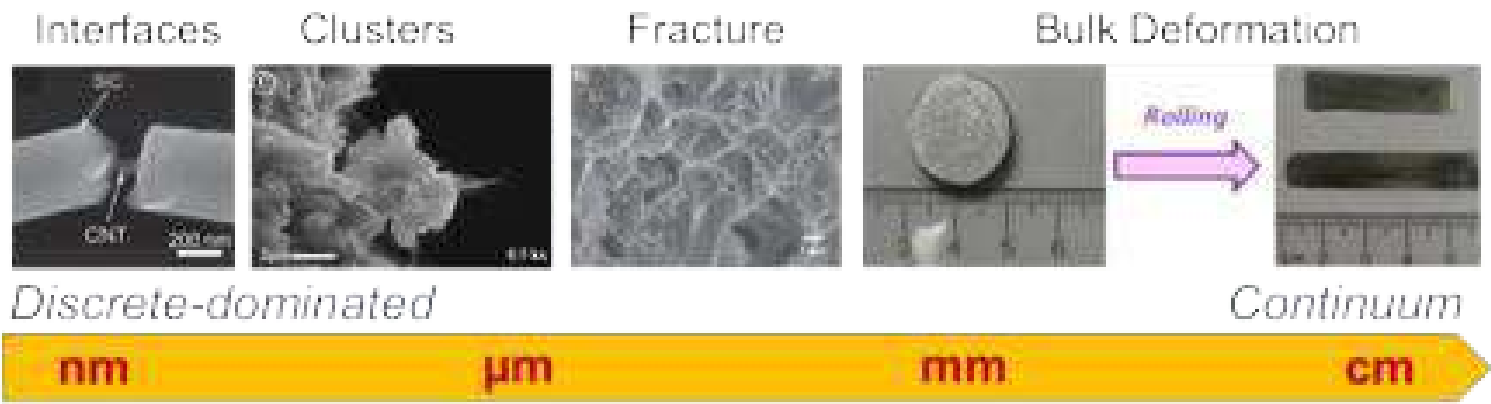

Figure 3.8. An illustration of multi-scale deformation phenomena in nanotube-reinforced $\mathrm{MMCs}^{29,31,32}$.

\subsection{Takeaway: Theoretical Framework to Meet Dissertation Objectives}

The insights provided by the theories are being applied for developing and interrogating BNNT-MMCs in this work. The following points succinctly summarize the key dissertation objectives which will be targeted:

- Homogeneous microstructure: The strong inter-tube interactions necessitate closer scrutiny of nanotube dispersion. Achieving effective dispersion takes the top priority, as it will influence composite fabrication and properties.

- Exceptional mechanical performance: The alignment of the filler phase is promising to utilize the full potential of BNNTs for mechanical reinforcement.

- Interface engineering: Metal-BNNT interface holds the key to mechanical strengthening. Chemical reactions are a useful means to tune the load-transfer within MMCs. 
- Scalable manufacturing: Effective integration of nanotubes in metal necessitates

a close examination of processing parameters and processing approaches. These parameters will greatly influence the metal-nanotube interactions during fabrication.

- Mechanistic understanding: Nanocomposite behavior cannot be completely explained by continuum treatment. A thorough understanding of mechanical phenomena at multiple length scales is necessary to design and optimize composite microstructures for targeted structural applications.

\section{References}

1. Gosens, I. et al. Impact of agglomeration state of nano- and submicron sized gold particles on pulmonary inflammation. Part. Fibre Toxicol. 7, 37 (2010).

2. Rumpf, H. Agglomeration. (John Wiley, 1962).

3. Tang, D.-M. et al. Mechanical properties of bamboo-like boron nitride nanotubes by in situ TEM and MD simulations: Strengthening effect of interlocked joint interfaces. ACS Nano 5, 7362-7368 (2011).

4. Yamaguchi, M., Meng, F., Firestein, K., Tsuchiya, K. \& Golberg, D. Powder metallurgy routes toward aluminum boron nitride nanotube composites, their morphologies, structures and mechanical properties. Mater. Sci. Eng. A 604, 9-17 (2014).

5. Matarredona, O. et al. Dispersion of single-walled carbon nanotubes in aqueous solutions of the anionic surfactant NaDDBS. J. Phys. Chem. B 107, 13357-13367 (2003).

6. Vaisman, L., Wagner, H. D. \& Marom, G. The role of surfactants in dispersion of carbon nanotubes. Adv. Colloid Interface Sci. 128-130, 37-46 (2006).

7. Islam, M. F., Rojas, E., Bergey, D. M., Johnson, A. T. \& Yodh, A. G. High weight fraction surfactant solubilization of single-wall carbon nanotubes in water. Nano Lett. 3, 269-273 (2003). 
8. Ryu, H. J., Cha, S. I. \& Hong, S. H. Generalized shear-lag model for load transfer in SiC/Al metal-matrix composites. J. Mater. Res. 18, 2851-2858 (2003).

9. Agarwal, A., Bakshi, S. R. \& Lahiri, D. Carbon Nanotubes: Reinforced Metal Matrix Composites. (CRC Press, Taylor \& Francis Group, 2011).

10. Coleman, J. N. et al. High-performance nanotube-reinforced plastics:

Understanding the mechanism of strength increase. Adv. Funct. Mater. 14, 791798 (2004).

11. Uchic, M. D., Dimiduk, D. M., Florando, J. N. \& Nix, W. D. Sample dimensions influence strength and crystal plasticity. Science (80-. ). 305, 986-989 (2004).

12. Kim, J. Y. \& Greer, J. R. Tensile and compressive behavior of gold and molybdenum single crystals at the nano-scale. Acta Mater. 57, 5245-5253 (2009).

13. Greer, J. R., Th, J. \& Hosson, M. De. Plasticity in small-sized metallic systems : Intrinsic versus extrinsic size effect. Prog. Mater. Sci. 56, 654-724 (2011).

14. Lahiri, D. et al. Insight into reactions and interface between boron nitride nanotube and aluminum. J. Mater. Res. 27, 2760-2770 (2012).

15. Zhou, W., Yamaguchi, T., Kikuchi, K., Nomura, N. \& Kawasaki, A. Effectively enhanced load transfer by interfacial reactions in multi-walled carbon nanotube reinforced Al matrix composites. Acta Mater. 125, 369-376 (2017).

16. Xu, J. Q., Chen, L. Y., Choi, H. \& Li, X. C. Theoretical study and pathways for nanoparticle capture during solidification of metal melt. J. Phys. Condens. Matter 24, 255304 (2012).

17. Israelachvili, J. N. Intermolecular and Surface Forces. (Oxford: Academic, 2011).

18. Uhlmann, D. R., Chalmers, B. \& Jackson, K. A. Interaction Between Particles and a Solid-Liquid Interface. J. Appl. Phys. 35, 2986-2993 (1964).

19. Chan, D. Y. C. \& Horn, R. G. The drainage of thin liquid films between solid surfaces. J. Chem. Phys. 83, 5311 (1985).

20. Yamaguchi, M. et al. Utilization of multiwalled boron nitride nanotubes for the reinforcement of lightweight aluminum ribbons. Nanoscale Res. Lett. 8, 3 (2013).

21. Assael, M. J. et al. Reference data for the density and viscosity of liquid aluminum and liquid iron. J. Phys. Chem. Ref. Data 35, 285-300 (2006).

22. Corcione, M. Empirical correlating equations for predicting the effective thermal conductivity and dynamic viscosity of nanofluids. Energy Convers. Manag. 52, 789-793 (2011). 
23. Wang, G., Yu, D., Mohan, R. V., Gbewonyo, S. \& Zhang, L. A comparative study of nanoscale glass filler reinforced epoxy composites: Electrospun nanofiber vs nanoparticle. Compos. Sci. Technol. 129, 19-29 (2016).

24. Lahiri, D., Bakshi, S. R., Keshri, A. K., Liu, Y. \& Agarwal, A. Dual strengthening mechanisms induced by carbon nanotubes in roll bonded aluminum composites. Mater. Sci. Eng. A 523, 263-270 (2009).

25. Quaresimin, M., Salviato, M. \& Zappalorto, M. Strategies for the assessment of nanocomposite mechanical properties. Compos. Part B Eng. 43, 2290-2297 (2012).

26. Singhal, S. K., Srivastava, A. K., Pasricha, R. \& Mathur, R. B. Fabrication of AlMatrix Composites Reinforced with Amino Functionalized Boron Nitride Nanotubes. J. Nanosci. Nanotechnol. 11, 5179-5186 (2011).

27. Golberg, D., Xu, F. F. \& Bando, Y. Filling boron nitride nanotubes with metals. Appl. Phys. A Mater. Sci. Process. 76, 479-485 (2003).

28. Pham, T. et al. A Universal Wet-Chemistry Route to Metal Filling of Boron Nitride Nanotubes. Nano Lett. 16, 320-325 (2016).

29. Boesl, B., Lahiri, D., Behdad, S. \& Agarwal, A. Direct observation of carbon nanotube induced strengthening in aluminum composite via in situ tensile tests. Carbon N. Y. 69, 79-85 (2014).

30. Mateos, A. J., Huang, W., Zhang, Y. W. \& Greer, J. R. Discrete-Continuum Duality of Architected Materials: Failure, Flaws, and Fracture. Adv. Funct. Mater. 29, 1806772 (2019).

31. Yang, Y. et al. Unveil the Size-Dependent Mechanical Behaviors of Individual CNT/SiC Composite Nanofibers by In Situ Tensile Tests in SEM. Small 12, 44864491 (2016).

32. Lahiri, D. et al. Boron nitride nanotubes reinforced aluminum composites prepared by spark plasma sintering: Microstructure, mechanical properties and deformation behavior. Mater. Sci. Eng. A 574, 149-156 (2013). 


\section{CHAPTER 4}

\section{MATERIALS, EXPERIMENTAL METHODOLOGIES AND ANALYSIS TECHNIQUES}

There are three key pillars of this research to achieve the end goal of engineering MMCs with brilliant mechanical performance: (i) use of scalable manufacturing techniques capable of processing meaningful volumes of composites, (ii) microstructure design at multiple hierarchical levels for effectively leveraging brilliant intrinsic properties of BNNT, and (iii) develop fundamental mechanistic insights into the role of processing approaches and microstructure design strategies on the overall mechanical properties. This chapter lays out the materials details, experimental methodologies and analysis techniques used in this work to meet these dissertation objectives.

\subsection{Boron Nitride Nanotube Precursor}

\subsubsection{Puffy Balls}

Extremely fine ( 5-10 nm diameter) and long (up to $200 \mu \mathrm{m})$ BNNTs are used in this research project (Figure 4.1a). The nanotubes are 1-5 walls thick, with 2 or 3 walls most common. They were obtained in the form of fibril balls (BNNT P1 Beta) from BNNT, LLC (Newport News VA, United States), shown in the inset. These fibril balls are extremely lightweight (tapping density $\sim 0.5 \mathrm{mg} / \mathrm{cm}^{3}$ ), and comprise of densely entangled nanotubes with a very high aspect ratio of $\sim 30,000-70,000$. They are fabricated using a high power laser-based method, producing crystalline, small diameter and ultra-long BNNTs under high pressure and high-temperature environment ${ }^{1}$. There are no elemental impurities in 
BNNTs prepared by this catalyst-free method; however, there were free boron and boron nitride nanoparticles in the BNNT fibril balls. Fourier transform infrared spectroscopy (FTIR) plot of as-received BNNT shows the characteristic h-BN peaks ${ }^{2-4}$ around $1356 \mathrm{~cm}^{-}$ ${ }^{1}$ and $799 \mathrm{~cm}^{-1}$ (Figure 4.1b).
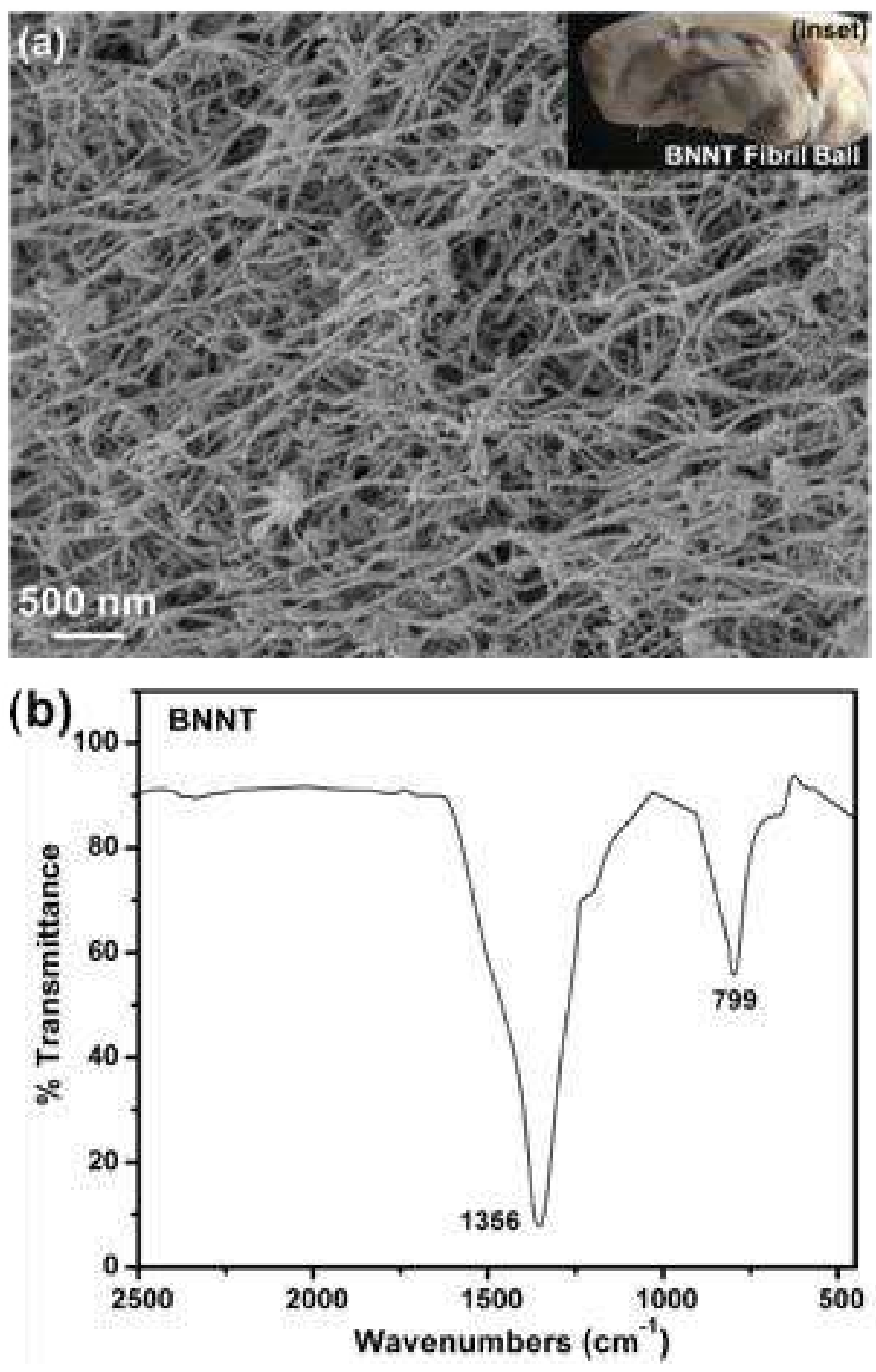

Figure 4.1. (a) FESEM image of the as-received dense cluster of nanotubes (inset shows the as received ball of entangled BNNTs), and (b) FTIR spectrum of as-received BNNTs. 


\section{Wet Chemistry Pathway for BNNT Dispersion}

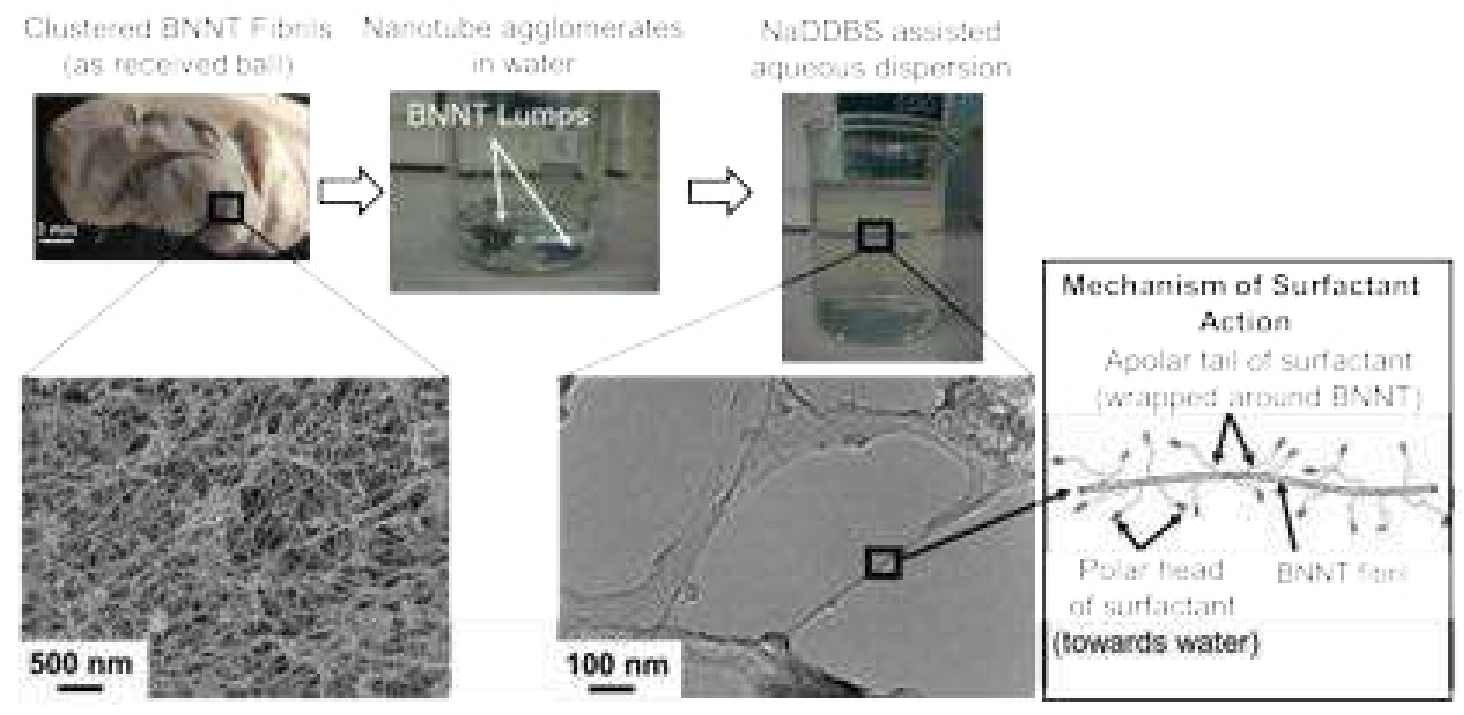

Figure 4.2. Sonication and surfactant-assisted BNNT de-agglomeration and dispersion in water.

\subsubsection{Dispersion of Nanotubes}

Due to the very large surface area of the nanotubes, these agglomerates are held together by strong inter-particle forces. For effective reinforcement effect, it is essential to have a uniform distribution of nanotubes in the metal matrix ${ }^{5}$. A wet chemistry approach employing an ionic surfactant was adopted to break these nanotube clusters and disperse them. The nanotube clusters and Sodium Dodecylbenzenesulfonate (NaDDBS, Sigma Aldrich, St. Louis, Missouri) surfactant were added to de-ionized water in a beaker. The mixture was bath sonicated for 12 hours. Sonication assists in breaking the nanotube agglomerates and NaDDBS aids in dispersing the nanotubes in water, such that they do not re-agglomerate ${ }^{6}$. A BNNT concentration of $10 \mathrm{mg} / \mathrm{mL}$ and BNNT:NaDDBS ratio of 1:10 were used for obtaining the dispersion. Transmission electron microscopy (TEM) of the 
aqueous solution showed separated nanotubes, with no agglomeration (Figure 4.2). Ionic surfactant molecule, due to the presence of both polar and apolar groups, adsorbs at the interface of BNNT and water and reduces the surface tension ${ }^{7}$. While the hydrophobic hydrocarbon tail wraps around the nanotube, the hydrophilic polar head protrudes into the water. This allows the water molecules to adhere to the otherwise unwettable nanotubes. Figure 4.2 schematically summarizes the wet chemistry approach adopted during the synthesis of the Al-BNNT nanocomposite.

\subsubsection{Buckypaper}

A freestanding buckypaper ${ }^{8}$ was used to probe the mechanical properties of BNNTs at elevated temperatures. The buckypaper comprises of a randomly oriented network of high aspect nanotubes (Figure 4.3a). The buckypaper has a very high degree of porosity of $80 \%$. The microstructure comprises of a large number of nodes, where multiple nanotubes intersect and make contact with each other (Figure 4.3b). The majority of the nodes are 4degree junctions, that is, 4 nanotube struts emanate from the node (encircled in yellow). Some of these junctions are characterized by 5 or more nanotube struts/ bundles emerging from the nodal junctions (encircled in red), suggesting a highly interconnected microstructure. The cross-section of the buckypaper is characterized by a multi-layer architecture, with multiple BNNT meshes stacked over each other (Figure 4.3c). This interconnected nanotube network provides structural stability to the buckypaper, as it can be folded and handled without breaking (shown in the inset to Figure 4.3a). 

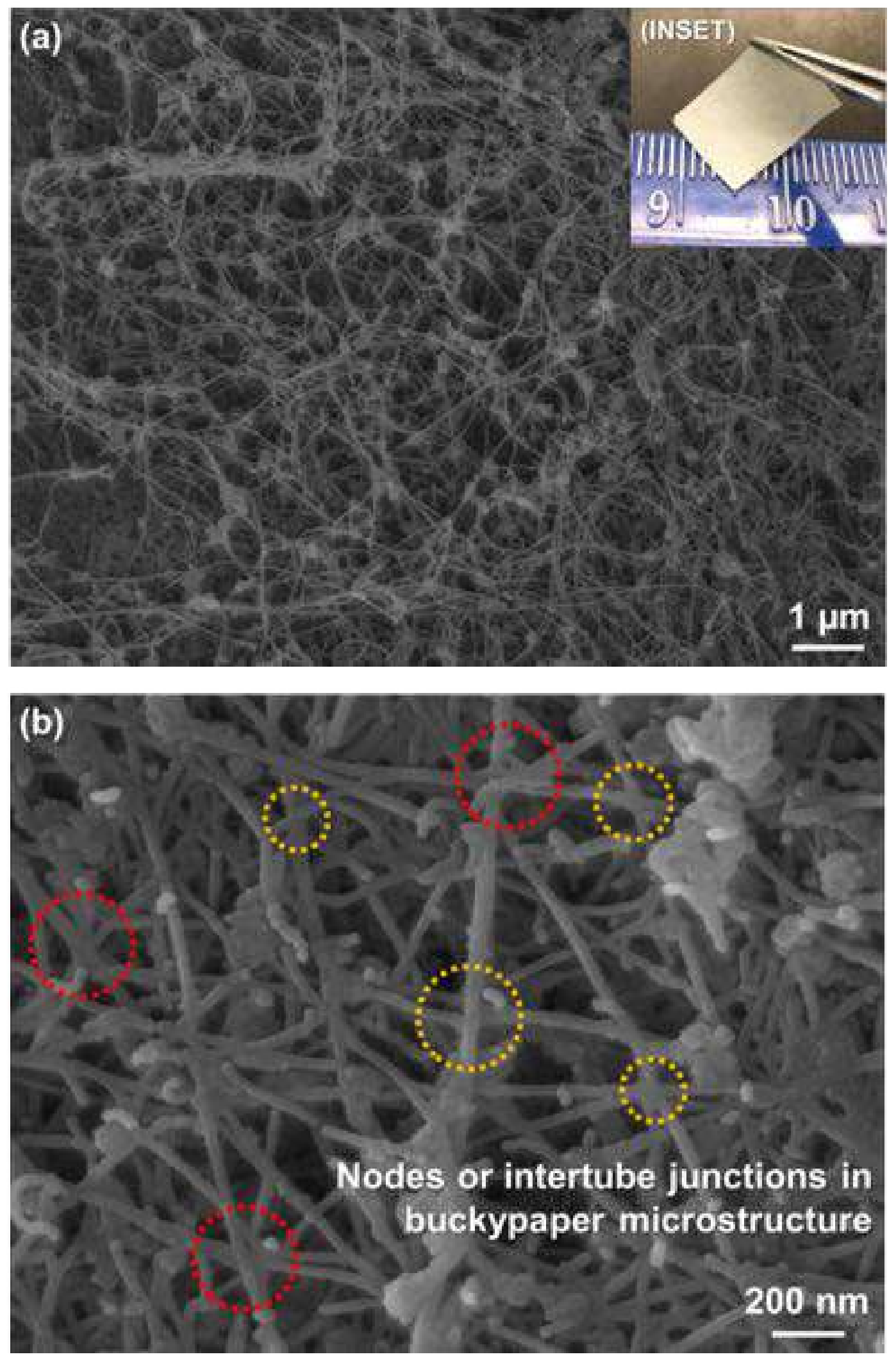


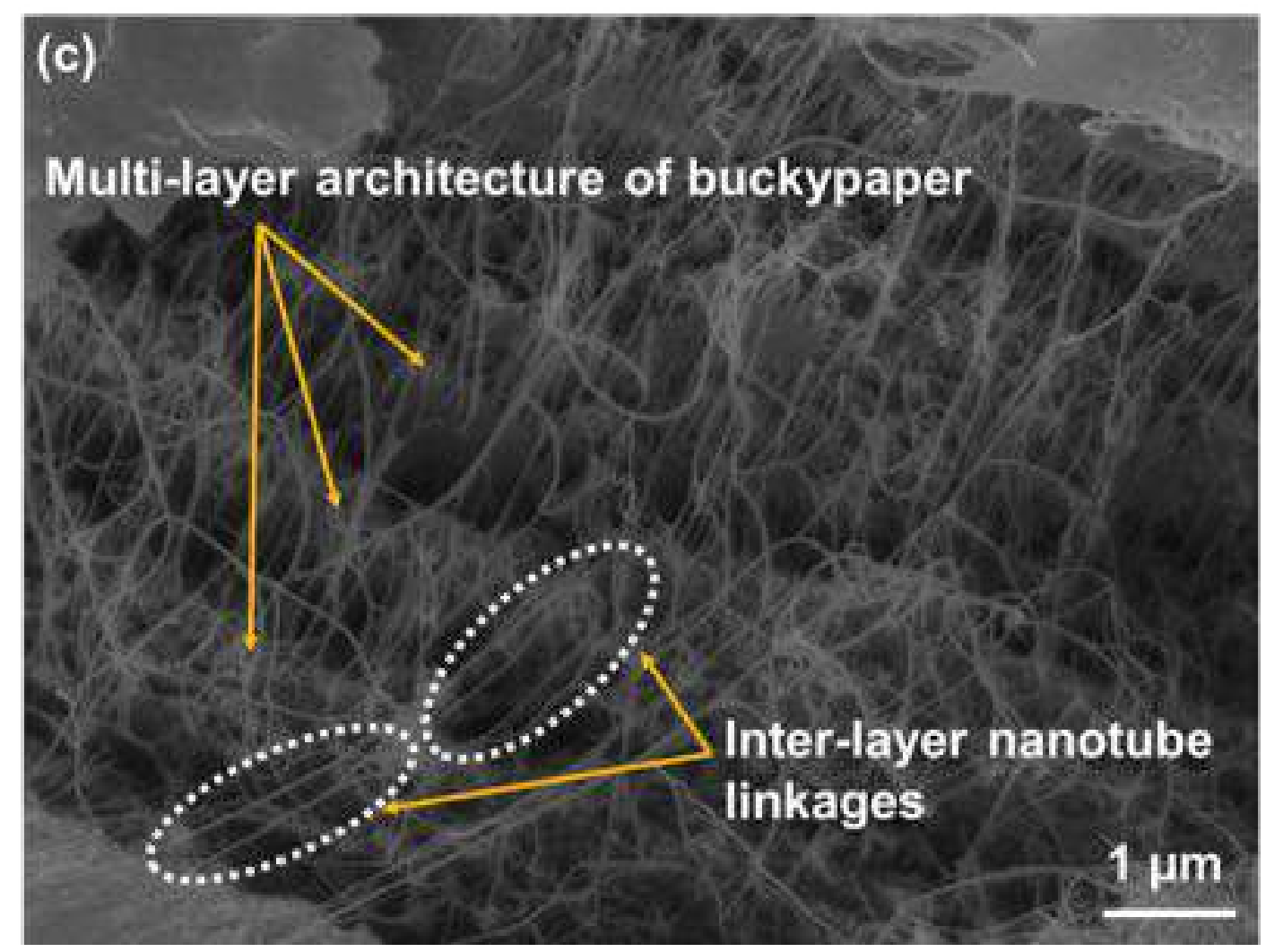

Figure 4.3. SEM micrographs of BNNT buckypaper microstructure: (a) a network of high aspect ratio nanotubes randomly aligned to form mesh-like architecture (inset shows a freestanding, foldable buckypaper), (b) high magnification micrograph of a network of nodes/ intertube junctions and nanotube struts, and (c) cross-section of the buckypaper comprising of multiple layers of BNNT meshes with inter-layer nanotube linkages providing structural support.

\subsection{Processing Approaches}

\subsubsection{Spark Plasma Sintering}

Spark plasma sintering (SPS) process was employed for pressure, and direct current (DC) assisted consolidation. High pressure and local electric discharge (between the powders and nanotubes) during SPS can promote rapid physical/chemical bonding ${ }^{9}$. The vacuum environment of SPS is also conducive to prevent high-temperature oxidation of metal 

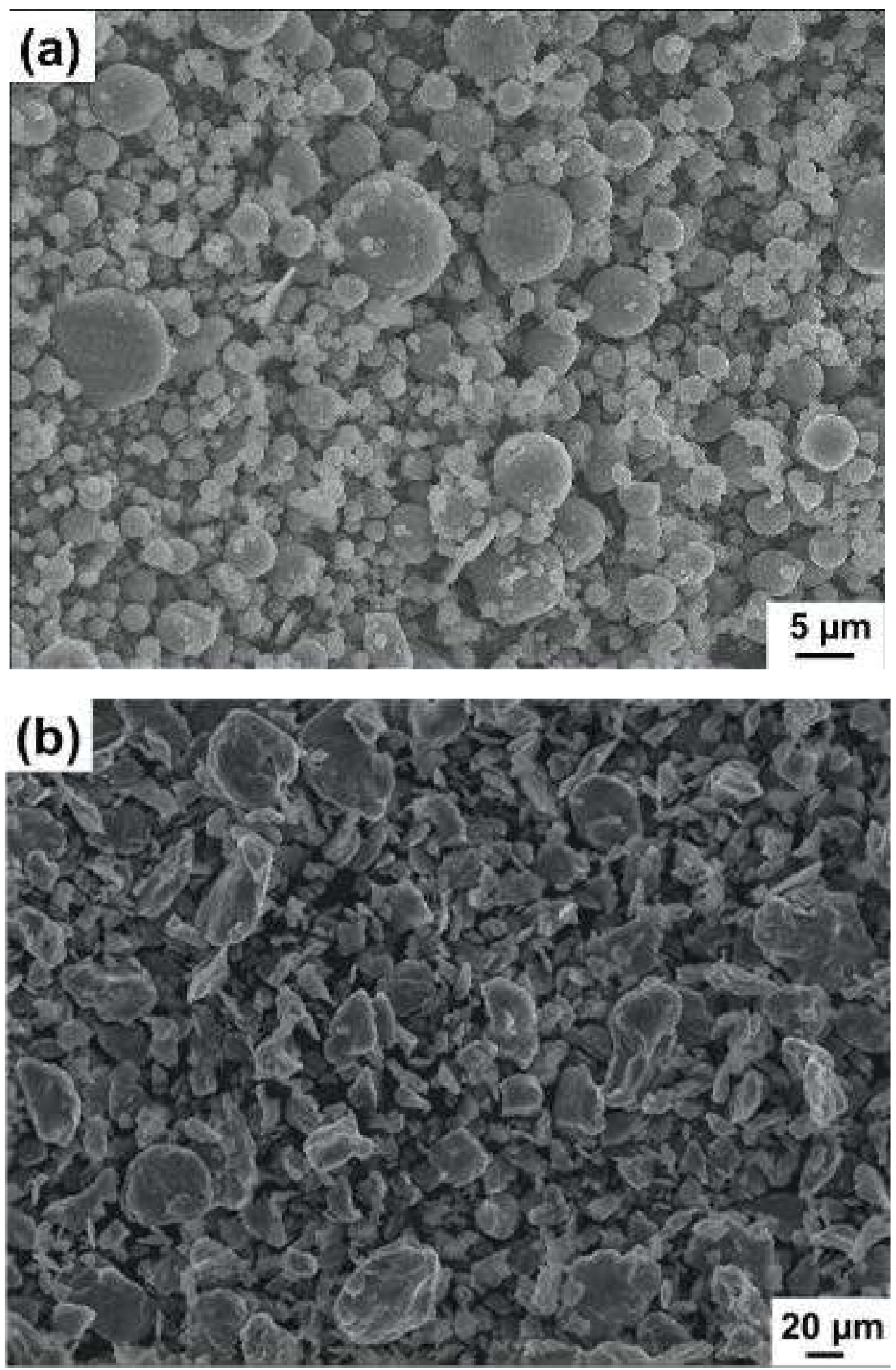

Figure 4.4. FESEM images of as-received (a) Al, and (b) AZ31 powders for fabricating BNNT-MMCs. 
powders. Figures 4.4a, b show the starting Al (H3, Valimet Inc., USA) and AZ31 magnesium alloy (Elektron - 21, -325 mesh, Magnesium Elektron, USA) powders used for fabricating the composites. Multi-layer composites were fabricated by SPS, such that a mat of BNNT was sandwiched between the layers of the metal matrix. BNNT mat was formed by cutting and stretching out a thin layer from the BNNT ball. This mat was sputter-coated with metal prior to integrating into sandwich-structured composites. The ballistic impact of metal on BNNT mat during sputtering is intended to create interfaces conducive to matrix-filler inter-layer bonding during processing.

Table 4.1 summarizes the SPS parameters used for sintering Al-BNNT and AZ31 $\mathrm{Mg}$ alloy-BNNT composites. It is noteworthy that the sintering of $\mathrm{Mg}$ and its alloys is challenging due to the native oxide layer on the powder particles and its limited plastic deformability ${ }^{10}$. Therefore, much higher sintering pressures were used.

Table 4.1. SPS processing parameters for consolidation of BNNT-MMC systems

\begin{tabular}{llllll}
\hline MMC System & Temperature & Pressure & Hold Time & Heating Rate & Die \\
\hline Al-BNNT & $575^{\circ} \mathrm{C}$ & $80 \mathrm{MPa}$ & 1 hour & $100^{\circ} \mathrm{C} / \mathrm{min}$ & Graphite \\
\hline AZ31-BNNT & $400^{\circ} \mathrm{C}$ & $400 \mathrm{MPa}$ & 10 minutes & $25^{\circ} \mathrm{C} / \mathrm{min}$ & $\mathrm{WC}$ \\
\hline
\end{tabular}

An additional rolling step was performed after sintering to obtain sheets of AlBNNT. The SPS pellet cold-rolled (two passes) at room temperature, with a $55 \%$ reduction in thickness. The composite displayed excellent deformability, with no cracking or disintegration due to cold rolling. Cold rolling was performed for twin objectives: (i) 
enhancing interfacial bonding, and (ii) aligning the long BNNTs sandwiched between Al layers. Dog-bone tensile specimens were cut out from the cold-rolled sheet by wire EDM. Schematic representation of the fabrication process and the pictures of the samples at each stage are shown in Figure 4.5. The Mg-BNNT composite could not be deformed into sheets. This is because of the inherent lack of plasticity in $\mathrm{Mg}$ alloys ${ }^{11}$. Therefore, microstructure study and mechanical investigations for Mg-BNNT reported in the next chapter are for sintered composite pellets.

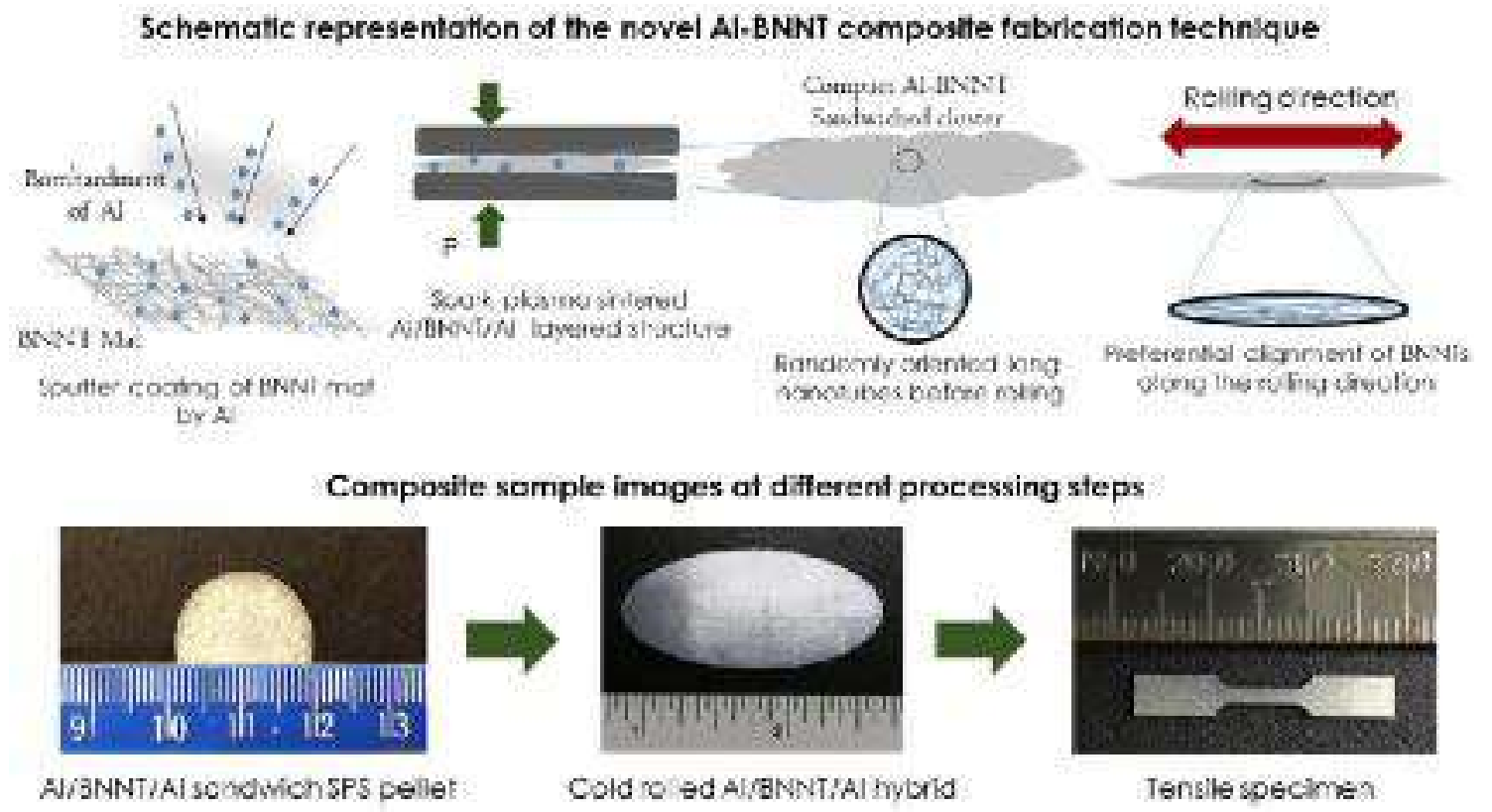

Figure 4.5. Schematic representation of the process of sputter coating, sandwiching and aligning long BNNTs within the Aluminum matrix, and the actual pictures of the composite sample at each stage. Similar sintering approach was adopted for AZ31-BNNT, except the final rolling step.

\subsubsection{Casting}

Casting is a classic and extensively utilized metal manufacturing approach. We fabricated Al-BNNT composites by casting route for exploring the integration of BNNT in Al via the 
solidification route. Nanotube fibers $(10 \mathrm{mg})$ were peeled from a BNNT fibril ball and were uniformly mixed with $\mathrm{Al}$ pellets $(20 \mathrm{~g})$ in a crucible. The mixture of $\mathrm{Al}$ pellets and BNNTs was heated to $700^{\circ} \mathrm{C}$ in a muffle furnace in air. The molten Al-BNNT mixture was gently stirred to ensure homogeneous composition. The mixture was soaked in the furnace at $700^{\circ} \mathrm{C}$ for 1 hour and was then slowly allowed to cool inside the closed furnace until it reaches ambient temperature (around 10 hours). The solidified composite was removed from the crucible and its surfaces were ground to remove crucible material stuck on the surfaces during casting. There were no macro-voids, inhomogeneities or prior pellet boundaries visible in the cast composite sample (Figure 4.6).

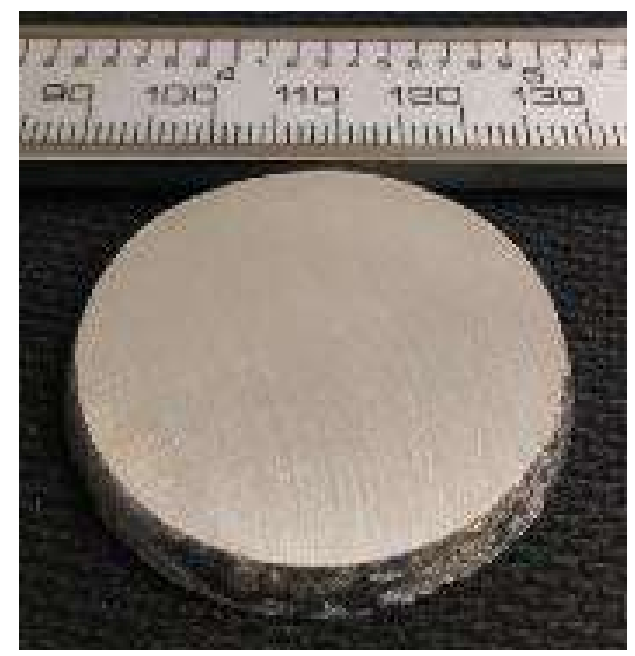

Figure 4.6. A solidified and ground Al-BNNT composite sample fabricated by casting.

\subsubsection{Plasma Spray}

Plasma spray is a thermal spray technique, where a feedstock powder is accelerated towards a substrate for layer-by-layer deposition and build-up of material. The particles 
experience elevated in-flight temperatures due to plasma ${ }^{12}$. The particle residence time in plasma is extremely short $\left(10^{-3} \mathrm{~s}\right)$, causing rapid solidification.

To prepare feedstock material, BNNT was first dispersed using the method discussed in Section 4.1.2. Spherical Al powder (H30, Valimet Inc., USA), with 90\% particles having diameter $<58 \mu \mathrm{m}$, was then added and thoroughly mixed in this aqueous BNNT dispersion using a magnetic stirrer. The Al-BNNT mixture was then heated to evaporate the water. The SEM micrograph in Figure 4.7 shows isolated, individual nanotubes wrapped around $\mathrm{Al}$ powder particles. The homogeneous Al-BNNT powder mixture was plasma sprayed using an SG 100 gun (Praxair Surface Technologies, Indianapolis, Indiana) on a $6061 \mathrm{Al}$ substrate. The gun was operated at a voltage and current of $35 \mathrm{~V}$ and $800 \mathrm{~A}$, respectively. Argon and Helium were used as the primary and secondary gases, respectively, with the respective flow rates of 56.6 and $59.5 \mathrm{slm}$.

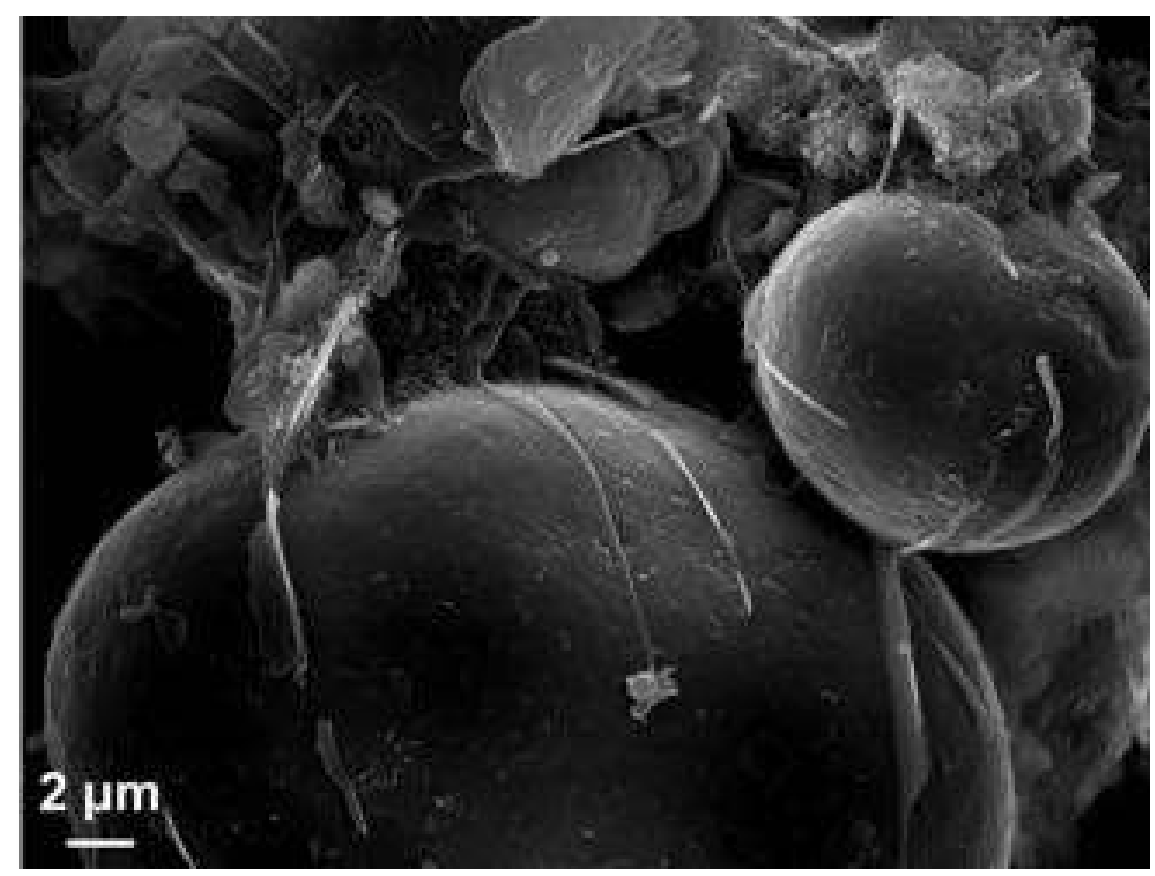

Figure 4.7. Dispersed Al-BNNT feedstock powder for plasma spray deposition obtained by the wet chemistry route. 


\subsection{Microstructure Evaluation}

\subsubsection{BNNT Integration and Distribution}

The composite samples were fractured for observing the internal microstructure. SEM imaging was performed using JEOL JSM-6330F field emission SEM (Tokyo, Japan), at operating voltages ranging from 10 to $30 \mathrm{kV}$ and working distances varying from 15-40 mm. High-resolution transmission electron microscopy (HRTEM) investigations were performed to interrogate the metal-nanotube interactions. TEM samples were prepared from the regions of interest by focused ion beam machining (JEOL-JIM 4500).

\subsubsection{Interfacial Reactions}

X-ray diffraction (XRD) was carried out to identify the phases in BNNT-MMC composites. XRD was performed using Siemens D-5000 X-ray diffractometer (Munich, Germany), based on $\mathrm{CuK}_{\alpha}$ radiation at an operating voltage and current of $40 \mathrm{kV}$ and $35 \mathrm{~mA}$, respectively. X-ray photoelectron spectroscopy (XPS) characterization was carried out for phase mapping in the composites using ThermoFischer Scientific ESCALAB-250Xi spectrometer (Waltham, USA). Monochromatic Al k $\alpha$ was used as the radiation source, operating at $300 \mathrm{~W}$ power, $15 \mathrm{kV}$ voltage and $20 \mathrm{~mA}$ current.

For observing and measuring the thickness of reaction products, HRTEM imaging of metal/BNNT interfacial regions was performed using Tecnai FEI F30 HRTEM at an operating voltage of $300 \mathrm{kV}$. Lattice spacing calculations were performed by fast and inverse fast Fourier transform (FFT \& IFFT) analysis using Digital Micrograph software (Gatan, Inc.). Elemental mapping is carried out by energy-dispersive X-ray spectroscopy 
for high-resolution chemical analysis of interfaces. Additionally, thermodynamic calculations were performed to quantify the Gibbs free energy change for interfacial chemical reactions. The Equilib module of FactSage thermochemical software was used for the analysis ${ }^{13}$. The experimental processing temperature, pressure, and material compositions were used for the calculations.

\subsection{Multi-scale Mechanical Characterization}

\subsubsection{Nanoscale Mechanical Properties}

Nanoindentation tests (TI 900 Triboindenter, Hysitron Inc., USA) were conducted to ascertain the effect of BNNT reinforcement locally in a composite microstructure. Nanoindentation response is highly sensitive to the state of the surface. Therefore, the samples were metallographically polished to remove any scratches and oxide layer prior to nanoindentation. The tests were performed using a sharp diamond Berkovich tip of $100 \mathrm{~nm}$ diameter. The tip was calibrated with a standard fused silica sample, by fitting the contact area with a polynomial function of contact depth. The tests were carried out in quasi-static load control mode, for a peak load of $2000 \mu \mathrm{N}$ and at a constant loading-unloading rate of $400 \mu \mathrm{N} / \mathrm{s}$. A short dwell time of $5 \mathrm{~s}$ at the peak load was programmed to prevent the nosing of the curve often seen during the onset of the unloading segment. The reduced elastic modulus $\left(E_{r}\right)$ was determined using Oliver Pharr method ${ }^{14}$ :

$$
E_{r}=\frac{1}{2} \frac{d F}{d h} \frac{\sqrt{\pi}}{\sqrt{A_{c}}}
$$


where $F$ is the indentation load, $h$ is the penetration depth, $A_{c}$ is the projected area of tipsample contact, and $d F / d h$ is called contact stiffness, computed at the point of onset of unloading. The elastic modulus of the composite $\left(E_{s}\right)$ was then be calculated using the following equation ${ }^{15}$ :

$$
\frac{1}{E_{r}}=\frac{\left(1-v_{S}^{2}\right)}{E_{S}}+\frac{\left(1-v_{i}^{2}\right)}{E_{i}}
$$

where $v$ is the Poisson's ratio, and the subscripts' and ' $\mathrm{i}$ ' stand for sample and indenter, respectively.

\subsubsection{Meso-scale Indentation Deformation}

The elastic-plastic zone during nanoindentation is extremely small, capturing the response of selected features in the microstructure. In order to understand the effect of multiple grains, nanotubes and interfacial reaction products on the mechanical response, we conducted meso-scale indentation. The penetration depths in these tests were varied from $\sim 150 \mu \mathrm{m}$ to $1 \mathrm{~mm}$. The tests were conducted using MTI SEMtester micromechanical stage (Albany, USA) with a $445 \mathrm{~N}$ capacity load cell and custom fixtures developed in-house. The test was performed in the displacement control mode. A Vickers diamond tip was used to perform the tests.

\subsubsection{Bulk-scale Mechanical Properties}

The uniaxial tensile test was carried out in MTI SEMtester, with a $445 \mathrm{~N}$ capacity load cell. The samples were loaded at a constant displacement rate of $1 \mathrm{~mm} / \mathrm{min}$. The test was 
conducted at the ambient temperature. The test video was recorded by Dino-Lite AM2111 digital microscope (New Taipei City, Taiwan) for strain measurement and analysis. The strain was computed by video correlation MATLAB program, developed in Hedrick Lab, University of North Carolina (Chapel Hill, NC) ${ }^{16}$. Determination of strain by video extensometry ensured that there are no slippage artifacts in the curve. For high-temperature tensile tests, a heating element was fitted in the tensile stage that heats the specimen during the entire duration of the test by direct contact. Post failure sample imaging was performed using field emission SEM to observe deformation characteristics and identify BNNTinduced strengthening mechanisms in the composite.

\subsubsection{In-situ Investigation of Deformation Mechanisms}

\section{A. High-Speed Camera Imaging}

One of the objectives of the study is to evaluate the deformation mechanisms associated with BNNT macroassemblies (like a sheet or a mat) to evaluate the feasibility of integrating such structures inside metal matrices. We examined the tensile deformation of a BNNT buckypaper with simultaneous high-speed camera imaging (IL5, Fastec Imaging, USA) to record the crack initiation and propagation phenomena at 5,000 frames per second.

\section{B. In-situ SEM Indentation}

Localized mechanical deformation of BNNT buckypaper and Ti-BNNT samples was captured by in-situ indentation testing (Picoindenter, Hysitron PI 87, Bruker, USA). The nanoindenter stage was installed inside a dual beam JEOL JIB-4500 focused ion beam/SEM system for in-situ imaging. For elevated temperature indentation testing, a 
high-temperature mechanical stage equipped with sample and probe heaters was used. The real-time high-resolution SEM videos corresponding to nanoindentation loading/ unloading were recorded.

- Testing of BNNT buckypaper: Micro-scale testing of BNNT buckypaper was carried out using a flat-ended cylindrical probe with $10 \mu \mathrm{m}$ diameter was used. The relatively larger probe is useful to capture the mechanical response due to the interactions between multiple nanotubes. The tests were performed in load-control mode with a peak indentation force of 5,000 $\mu \mathrm{N}$. Cyclic tests were also performed by programming 50 loading-unloading cycles. These mechanical investigations were performed as a function of temperature, from room temperature up to $500^{\circ} \mathrm{C}$.

- Testing of Ti-BNNT composites: The nanocomposite samples were tested using a fine cube corner tip with $40 \mathrm{~nm}$ radius. The cube corner tip is effective for inducing instantaneous plasticity and it also provides an enhanced field of view owing to the acute face angle of $35.26^{\circ}$. The tests were conducted in displacement-control mode, with indentation depths varying from $500 \mathrm{~nm}$ to $5,000 \mathrm{~nm}$, to capture multi-scale deformation mechanisms activated in the microstructure.
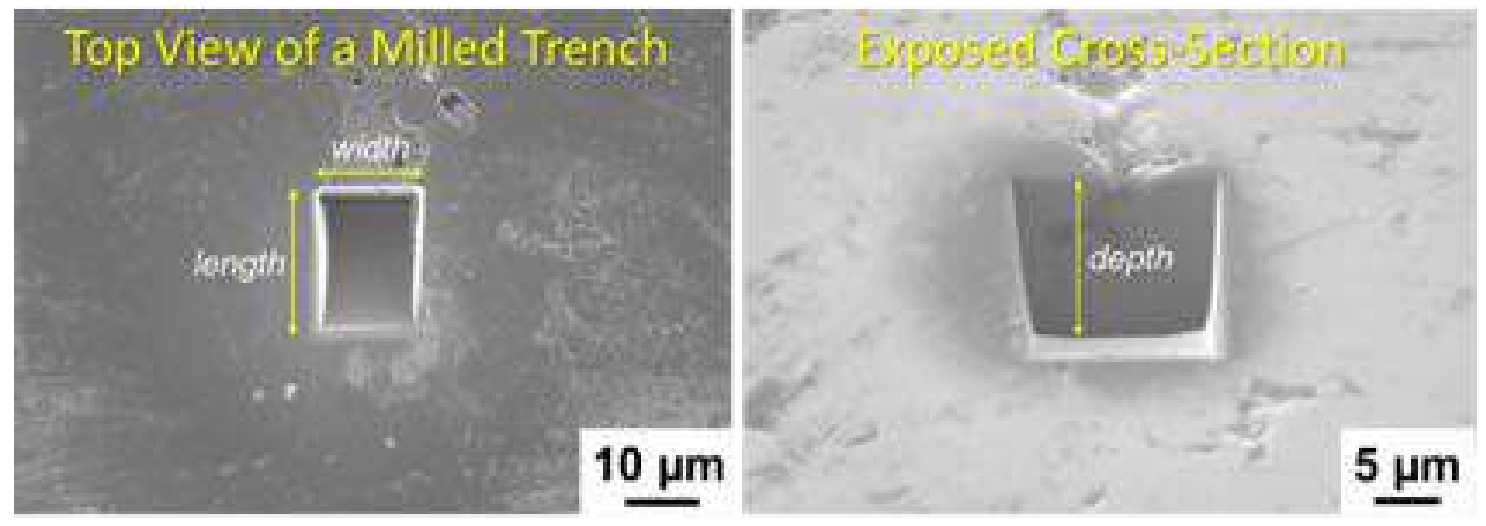

Figure 4.8. Focused ion beam milling of Ti-BNNT composite to expose the internal structure for observing the deformation mechanisms. 
Indentation testing reveals surface-level mechanisms only, and the internal mechanisms/ structural response cannot be observed. To obtain better visualization, we created trenches by FIB milling to expose the internal structure prior to indentation (Figure 4.8). $\mathrm{A} \mathrm{Ga}^{+}$ion beam was used for milling, with beam current varying from $2,000-5,000$ pA and beam diameter ranging from 200 to $300 \mathrm{~nm}$. The trenches were about $8-15 \mu \mathrm{m}$ wide, $15-23 \mu \mathrm{m}$ long and 5-10 $\mu \mathrm{m}$ deep. In-situ indentation tests were then performed near the edge of the trench to observe the sub-surface mechanisms in real-time. The critical stresses to active different mechanisms were calculated based on the load-displacement response.
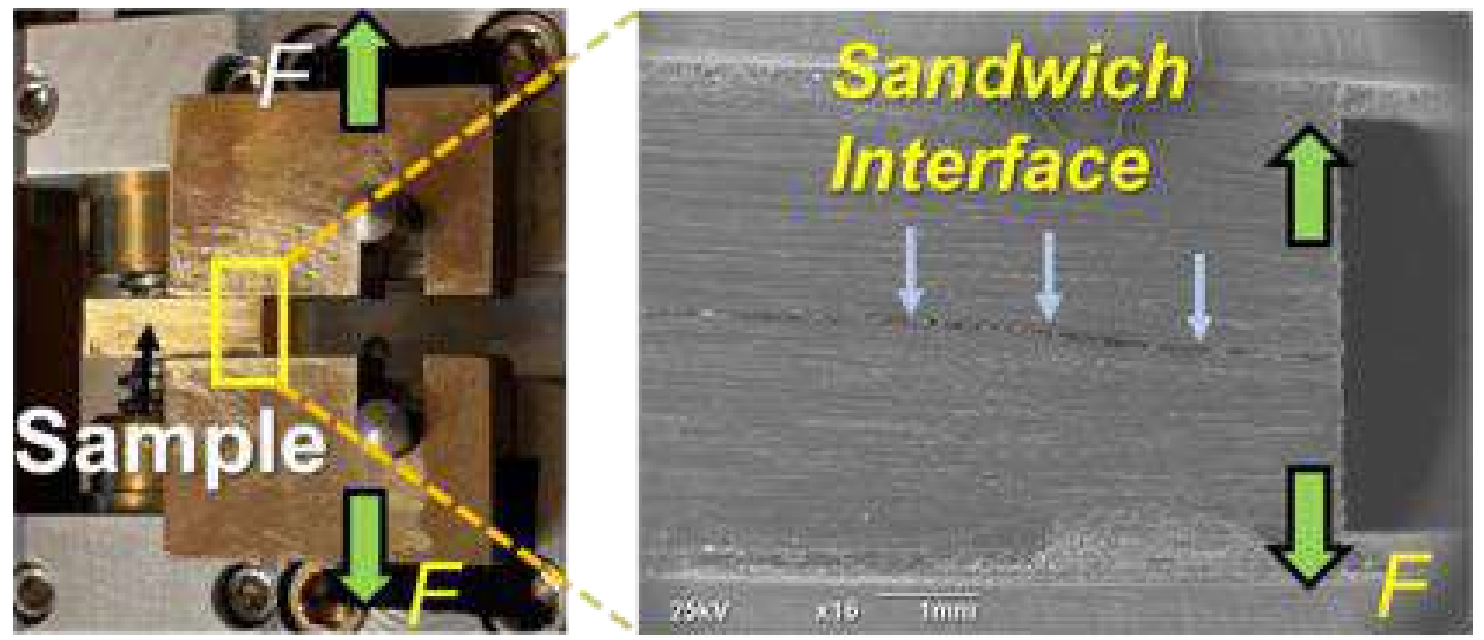

Figure 4.9. In-situ double cantilever test set-up for examining strengthening mechanisms at $\mathrm{Mg}-\mathrm{BNNT}$ interface in a sandwich-structured composite.

\section{In-situ SEM Double Cantilever Testing}

An in-situ double cantilever experimental setup was used to examine the mechanisms associated with BNNT reinforcement in a sandwich structured composite. A 
micromechanical stage (MTI SEMtester) with custom-designed fixtures was used to study failure in the composite (Figure 4.9). Mechanical loading led to interface deformation and debonding, and the associated mechanisms were imaged in real-time inside SEM.

\section{Digital Image Correlation Analysis}

Insights into microstructure deformation were developed by performing strain analysis of the real-time deformation snapshots using VIC-2D digital image correlation (DIC) software (Correlated Solutions, USA). In DIC analysis, the real-time snapshots of the microstructure (during deformation) are compared/ analyzed with respect to the starting snapshot, to capture the changes in specimen surface incrementally as the force is applied ${ }^{17}$. The software tracks and correlates the pixels in the deformed snapshots with respect to the starting image. This relative comparison aids in determining full-field 2D strain map, showing the spatial distribution of strains in the microstructure as a function of mechanical loading $^{18,19}$.

\subsection{Remarks}

The methodologies discussed in this chapter intend to establish the processing-structuremechanics correlation for this new class of composite materials. High-resolution imaging and analysis are aimed to develop a detailed understanding of microstructure evolution using the scalable manufacturing techniques discussed in the chapter. Further, advanced in-situ mechanical investigations will unravel novel deformation characteristics previously 
unexplored in these hierarchical nanocomposites. The experimental findings are presented in great detail in the next 3 chapters.

\section{References}

1. Smith, M. W. et al. Very long single-and few-walled boron nitride nanotubes via the pressurized vapor/condenser method. Nanotechnology 20, 505604 (2009).

2. Ferreira, T. H., Silva, P. R. O., Santos, R. G. \& Sousa, E. M. B. A Novel Synthesis Route to Produce Boron Nitride Nanotubes for Bioapplications. J. Biomater. Nanobiotechnol. 02, 426-434 (2011).

3. Liao, Y. et al. Oxidative Etching of Hexagonal Boron Nitride Toward Nanosheets with Defined Edges and Holes. Sci. Rep. 5, 14510 (2015).

4. Shin, H. et al. Covalent Functionalization of Boron Nitride Nanotubes via Reduction Chemistry. ACS Nano 9, 12573-12582 (2015).

5. Agarwal, A., Bakshi, S. R. \& Lahiri, D. Carbon Nanotubes: Reinforced Metal Matrix Composites. (CRC Press, Taylor \& Francis Group, 2011).

6. Zheng, M. et al. Radial elasticity of multi-walled boron nitride nanotubes. Nanotechnology 23, 095703 (2012).

7. Vaisman, L., Wagner, H. D. \& Marom, G. The role of surfactants in dispersion of carbon nanotubes. Adv. Colloid Interface Sci. 128-130, 37-46 (2006).

8. Kim, K. S. et al. Polymer nanocomposites from free-standing, macroscopic boron nitride nanotube assemblies. RSC Adv. 5, 41186-41192 (2015).

9. Fontoura, L., Nautiyal, P., Loganathan, A., Boesl, B. \& Agarwal, A. NacreInspired Graphene/Metal Hybrid by In Situ Cementation Reaction and Joule Heating. Adv. Eng. Mater. 20, 1800518 (2018).

10. Wolff, M., Ebel, T. \& Dahms, M. Sintering of magnesium. Adv. Eng. Mater. 12, 829-836 (2010).

11. Trang, T. T. T. et al. Designing a magnesium alloy with high strength and high formability. Nat. Commun. 9, 2522 (2018).

12. Bhusal, S. et al. A computational approach for predicting microstructure and mechanical properties of plasma sprayed ceramic coatings from powder to bulk. Surf. Coatings Technol. 374, 1-11 (2019). 
13. Bale, C. W. et al. Reprint of: FactSage thermochemical software and databases, 2010-2016. Calphad Comput. Coupling Phase Diagrams Thermochem. 55, 1-19 (2016).

14. Oliver, W. C. \& Pharr, G. M. An improved technique for determining hardness and elastic modulus using load and displacement sensing indentation experiments. J. Mater. Res. 7, 1564-1583 (1992).

15. C. Fisher-Cripps, A. Nanoindentation. (Springer, 2005). doi:10.1007/978-1-44199872-9

16. Hedrick, T. L. Software techniques for two- and three-dimensional kinematic measurements of biological and biomimetic systems. Bioinspiration and Biomimetics 3, 034001 (2008).

17. Nautiyal, P., Mujawar, M., Boesl, B. \& Agarwal, A. In-situ mechanics of 3D graphene foam based ultra-stiff and flexible metallic metamaterial. Carbon N. Y. 137, 502-510 (2018).

18. Embrey, L. et al. Three-Dimensional Graphene Foam Induces Multifunctionality in Epoxy Nanocomposites by Simultaneous Improvement in Mechanical, Thermal, and Electrical Properties. ACS Appl. Mater. Interfaces 9, 39717-39727 (2017).

19. Nautiyal, P., Zhang, C., Champagne, V. K., Boesl, B. \& Agarwal, A. In-situ mechanical investigation of the deformation of splat interfaces in cold-sprayed aluminum alloy. Mater. Sci. Eng. A 737, 297-309 (2018). 


\section{CHAPTER 5}

\section{PHYSICAL, CHEMICAL AND MECHANICAL CHARACTERISTICS OF BORON NITRIDE NANOTUBE AT ELEVATED TEMPERATURES}

Despite the fanfare around superior oxidation resistance and high temperature endurance, there is very little understanding of the physical, chemical and mechanical behavior of BNNT at elevated temperatures. While it is known that BNNT reacts to form Boron Trioxide $\left(\mathrm{B}_{2} \mathrm{O}_{3}\right)$ around $750-900^{\circ} \mathrm{C}^{1}$, the transformation mechanisms remain elusive. Being a 1-D nanomaterial, BNNT is anisotropic with strong directionality in its properties. This makes the understanding of morphological transformations associated with oxidation critical for mechanical reinforcement. The effect of high temperature exposure on surface chemistry is particularly important for engineering strong interfaces in MMCs. In this chapter, the findings on oxidative transformations and mechanical deformation of high aspect ratio $(\sim 30,000-70,000)$ BNNT assemblies are presented. In-situ mechanical investigations are performed to observe the deformation behavior of BNNTs in real-time. These mechanical investigations are performed up to $750^{\circ} \mathrm{C}$, which is the temperature around which the major structural transformations/ oxidative degradation of BNNT initiate.

\subsection{Thermal Transformations in Boron Nitride Nanotubes}

\subsubsection{Simultaneous Thermal Analysis}

Thermal analysis of nanotube clusters was performed by simultaneous Thermogravimetric Analysis (TGA) and Differential Scanning Calorimetry (DSC) tests up to $1000^{\circ} \mathrm{C}$. Major 


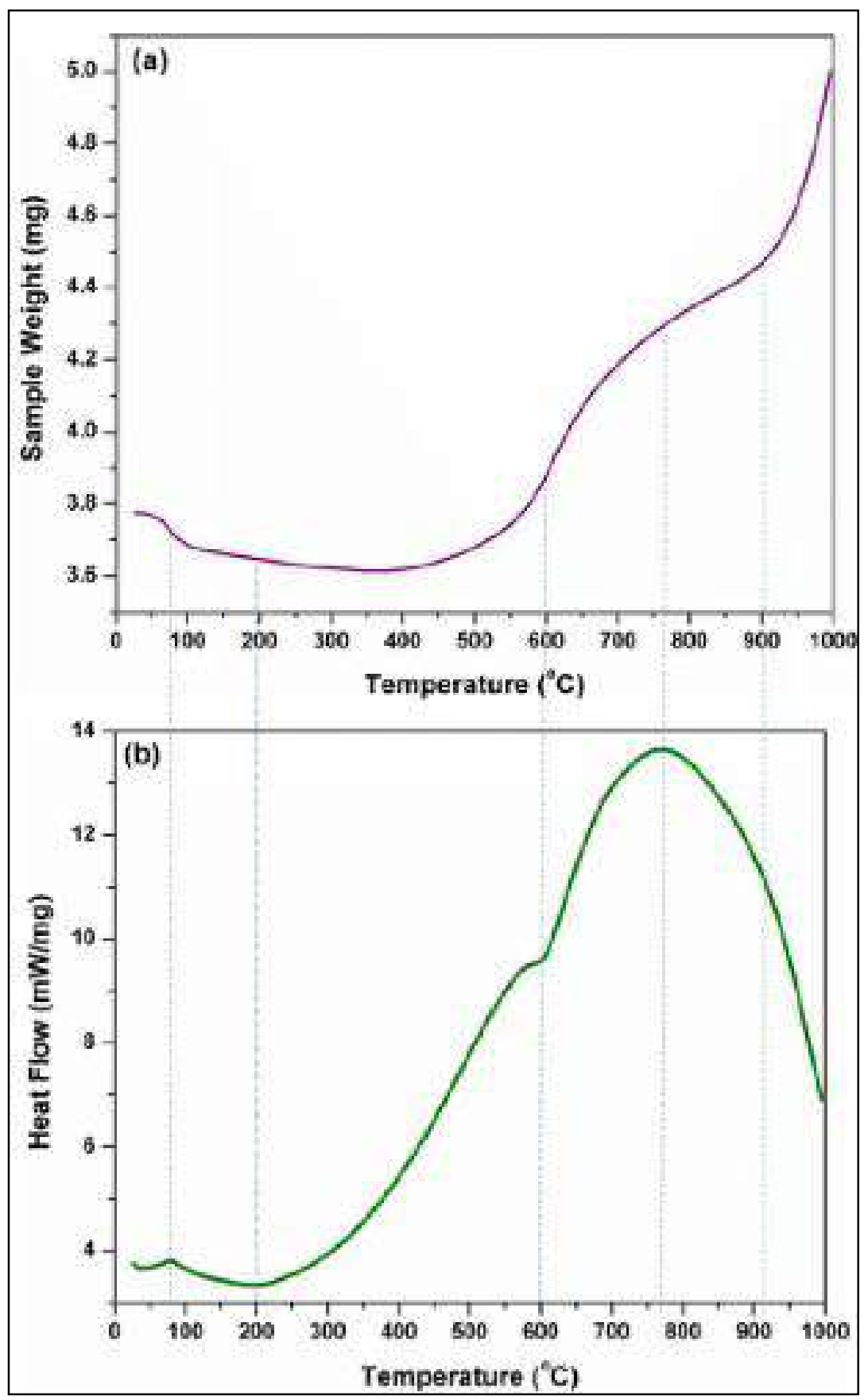

Figure 5.1. (a) Weight change of BNNT, and (b) heat required to increase the temperature of the BNNT sample during TGA-DSC, as a function of temperature.

peaks/ transition points are marked in TGA (Figure 5.1a) and DSC (Figure 5.1b) curves. There is an abrupt drop in weight initially, until $\sim 80^{\circ} \mathrm{C}$, after which the weight loss becomes 
more gradual. This transition is characterized by a sharp peak in the DSC curve in the beginning, which is associated with the loss of moisture in the sample. Vaporization is an endothermic process, which explains the peak in the DSC curve. There is a dominant peak at $\sim 750^{\circ} \mathrm{C}$, signifying major phase transition. A continuous weight gain trend is seen in the TGA curve in this regime, which can be related to the initiation of BNNT oxidation. Generalized Gradient Approximation (GGA) calculations performed by Zhang et al. for $(3,0)$ BNNT predicted lower oxidation energy barrier of $0.138 \mathrm{eV} /$ atom, which corresponds to $\sim 790^{\circ} \mathrm{C}^{2}$. The possibility of the oxidation reaction is subsequently probed by XRD, FTIR and EDS analysis.

\subsubsection{Phase Transformations}

X-Ray Diffraction (XRD) analysis of BNNT before and after TGA-DSC thermal treatment up to peak temperatures of 500,750 and $1000^{\circ} \mathrm{C}$ was performed to closely monitor the phase evolution and the progression of the oxidation reaction (Figure 5.2). The presence of the characteristic $\mathrm{h}-\mathrm{BN}$ peaks for all the temperatures indicates the survival of $\mathrm{h}-\mathrm{BN}$ phase up to $1000^{\circ} \mathrm{C}$. However, the peak intensity begins decreasing from $750^{\circ} \mathrm{C}$, indicating the oxidative transformation of the $\mathrm{BN}$ phase. There is no $\mathrm{B}_{2} \mathrm{O}_{3}$ phase observed at $500^{\circ} \mathrm{C}$. A Peak corresponding to $\mathrm{B}_{2} \mathrm{O}_{3}$ was detected for the first time in the BNNT sample heated up to $750^{\circ} \mathrm{C}$. This is in agreement with the DSC curve (Figure 5.1b), where a peak value was noticed at $\sim 750^{\circ} \mathrm{C}$ and can be attributed to the initiation of h-BN transformation to $\mathrm{B}_{2} \mathrm{O}_{3}$. The intensity of the $\mathrm{B}_{2} \mathrm{O}_{3}$ signal is enhanced at $1000^{\circ} \mathrm{C}$, where other $\mathrm{B}_{2} \mathrm{O}_{3}$ peaks are also visible (Figure 5.2), suggesting a greater concentration of boron trioxide due to the 


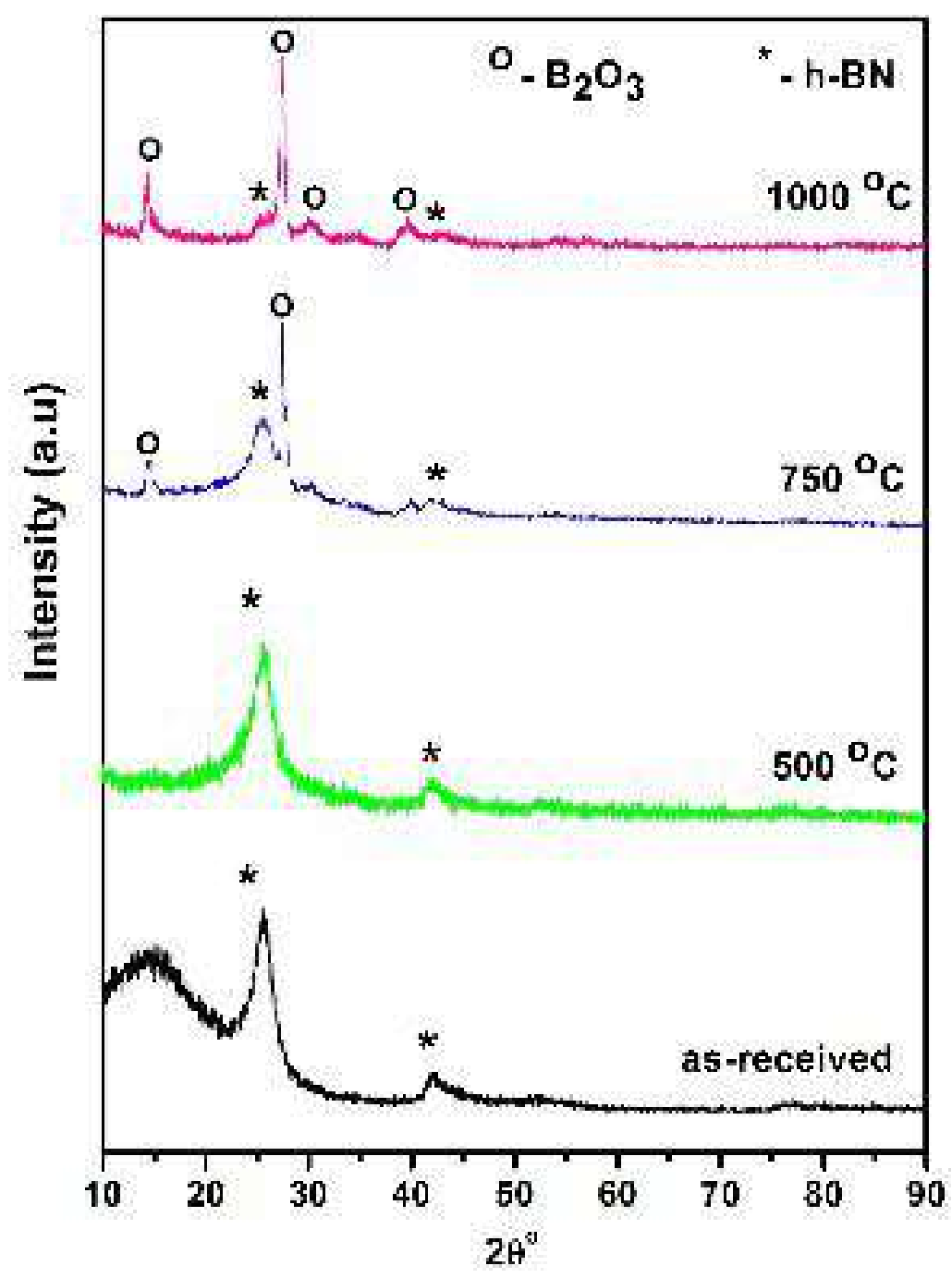

Figure 5.2. Post thermal analysis phase characterization of BNNT for different TGA-DSC maximum temperatures by X-Ray Diffraction.

accelerated rate of oxidation at high temperatures. This is attested by the TGA curve (Figure 5.1a), where there is a rapid increase in weight gain after $900^{\circ} \mathrm{C}$ (characterized by a steep rise in TGA slope). The weight gain can be explained by the higher density ( $\sim 2.46$ $\left.\mathrm{g} / \mathrm{cm}^{3}\right)$ of $\mathrm{B}_{2} \mathrm{O}_{3}$, as compared to h-BN $\left(\sim 2.1 \mathrm{~g} / \mathrm{cm}^{3}\right)$. In fact, hollow BNNT fibrils have been reported to have an unusually low density of $\sim 1.38 \mathrm{~g} / \mathrm{cm}^{3}$. This explains more than $31 \%$ 
weight gain at the end of TGA $\left(1000^{\circ} \mathrm{C}\right)$ with respect to as-received BNNTs noticed in Figure 5.1a. The disintegration of lightweight nanotubes at high temperatures and formation of comparatively denser non-tubular $\mathrm{BN}$ based phase and $\mathrm{B}_{2} \mathrm{O}_{3}$ leads to the overall weight gain.

\subsubsection{Oxidative Functionalization}

FTIR plots in Figure 5.3 reveal characteristic B-N stretching $\left(\sim 1353-1363 \mathrm{~cm}^{-1}\right)$ and bending modes $\left(\sim 792-799 \mathrm{~cm}^{-1}\right)^{4-6}$ for the entire temperature range investigated, showcasing the impressive oxidation resistance of these high aspect ratio nanotubes. It is interesting to note that the peaks corresponding to the B-O band $\left(1192 \mathrm{~cm}^{-1}\right)$ and O-B-O bond $\left(550 \mathrm{~cm}^{-1}\right)$ appear in BNNT treated at $500^{\circ} \mathrm{C}$. However, there is no $\mathrm{B}_{2} \mathrm{O}_{3}$ phase detected in XRD for this condition (Figure 5.2). Such behavior can be attributed to chemisorption of $\mathrm{O}_{2}$ on $\mathrm{B}$ atoms of nanotubes at $500^{\circ} \mathrm{C}$, without any free oxide formation. At 750 and $1000^{\circ} \mathrm{C}$, an additional peak corresponding to B-O stretching $\left(1025-1030 \mathrm{~cm}^{-1}\right)$ is detected. At these temperatures, $\mathrm{B}_{2} \mathrm{O}_{3}$ formation has been confirmed from XRD. Therefore, $\mathrm{BN}-\mathrm{O}_{2}$ interactions are not just limited to surface adsorption; rather, an oxidation reaction takes place to form boron trioxide. In addition, peaks corresponding to B-OH (3206-3220 $\left.\mathrm{cm}^{-1}\right)$ were also detected. This peak is very weak at room temperature (in as-received material) and could be due to moisture. However, at elevated temperatures, there is a marked increase in peak intensity. This suggests $\mathrm{B}-\mathrm{OH}$ bond formation. This is corroborated by the gradual increase in weight in the TGA curve (Figure 5.1a), suggesting 
progressive attachment of $-\mathrm{OH}$ functional group to $\mathrm{h}-\mathrm{BN}$ as the temperature increases before $\mathrm{B}_{2} \mathrm{O}_{3}$ formation initiates.

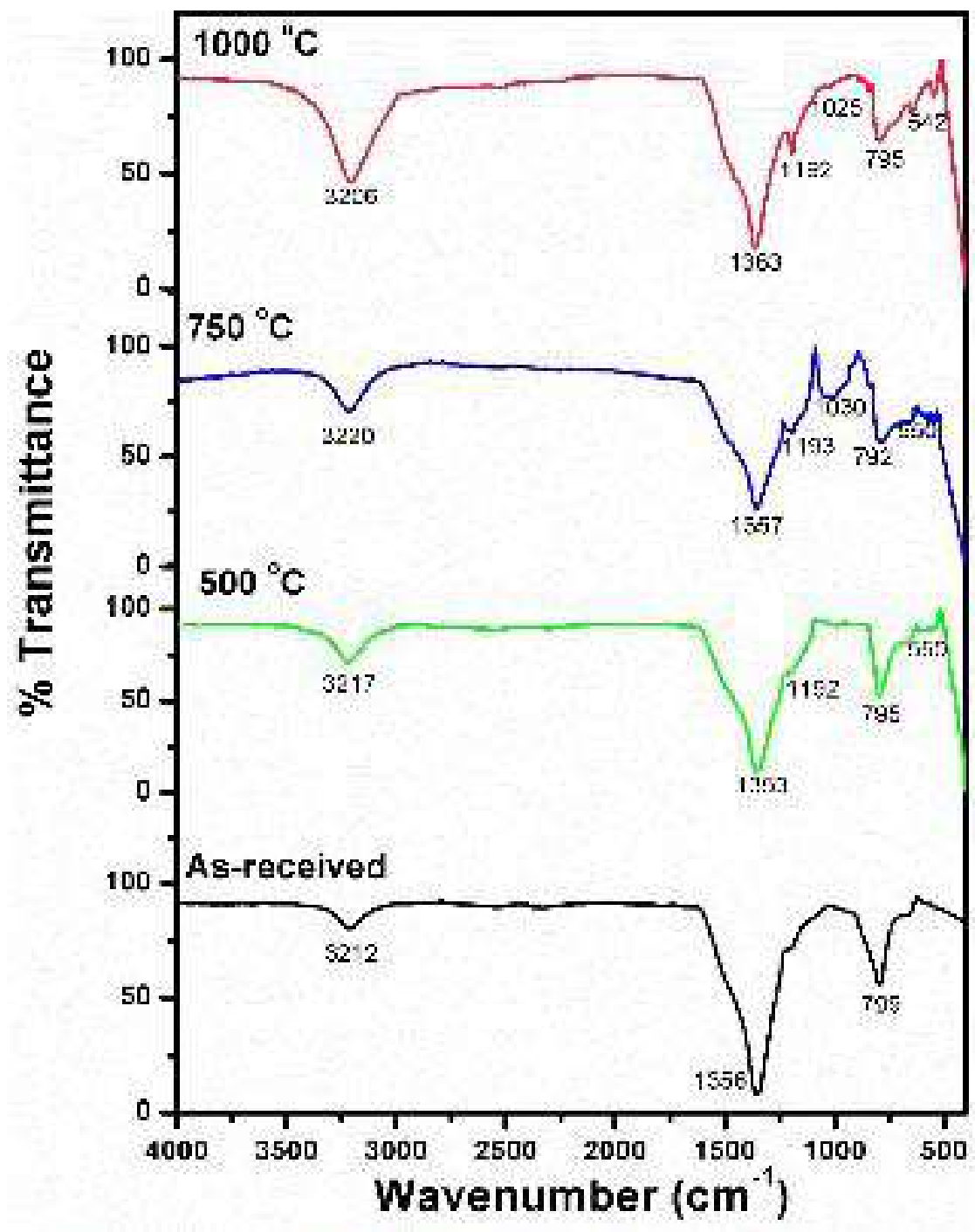

Figure 5.3. Fourier Transform Infrared Spectrum (FTIR) of BNNT sample subjected to TGA-DSC for varying temperatures, ranging from room temperature to $1000^{\circ} \mathrm{C}$.

\subsubsection{Morphological Transformations}

Transformations taking place in shape, morphology, and elemental composition of BNNT as a function of TGA-DSC temperature were examined by scanning electron microscopy 
(SEM) and Energy Dispersive Spectroscopy (EDS). Figure 5.4a shows the BNNTs at room temperature, consisting of a densely entangled network of fine and long nanotubes. Very small spherical particles can also be seen. These are possibly B and BN nanoparticles, which are reported in BNNTs synthesized by HTP technique ${ }^{7}$. After heating up to $500^{\circ} \mathrm{C}$, these spherical nodules were observed to grow in size as large as $400 \mathrm{~nm}$ (Figure 5.4b). Some of the nodules were attached to the tubes (encircled in green), while others grow in isolation from BNNTs. EDS examination of nodules revealed the presence of $\mathrm{O}$ in these locations. The presence of B-O bonds detected in FTIR (Figure 5.3) suggests that the growth of these nodules is related to the chemisorption of $\mathrm{O}_{2}$, and subsequent surface reaction on the nanoparticles.

The heating of BNNTs to $750^{\circ} \mathrm{C}$ resulted in the formation of thin $2 \mathrm{D}$ plates, while most of the nanotubes remain untransformed (Figure 5.4c). This shows that some of the BNNTs unwrap at elevated temperature to form planar ribbons, which coalesce to form $2 \mathrm{D}$ plates. At $1000^{\circ} \mathrm{C}$, the thickness of the stacked plates further increases (Figure 5.4d), suggesting the accelerated oxidative transformation of nanotubes. This is attested by the TGA curve (Figure 5.1a), where there is a steep increase in weight gain after $900^{\circ} \mathrm{C}$. There were no spherical nodules in 750 and $1000^{\circ} \mathrm{C}$ treated samples, indicating complete oxidation of the nanoparticles to form $\mathrm{B}_{2} \mathrm{O}_{3}$. XRD and FTIR results (Figures 5.2 and 5.3) suggest the survival of the h-BN phase even after the BNNT transform into the plate structure. Oxygen was detected on the EDS examination of these plates (Figure 5.4e). Based on FTIR and EDS results, it is clear that these 2D plates have B-O bonds, in addition to h-BN bonds. Moreover, B-OH bonds were also detected in FTIR (Figure 5.3). Therefore, these $2 \mathrm{D}$ plates, characterized by $\mathrm{B}-\mathrm{O}$ and $\mathrm{B}-\mathrm{OH}$ linkages attached to an array 

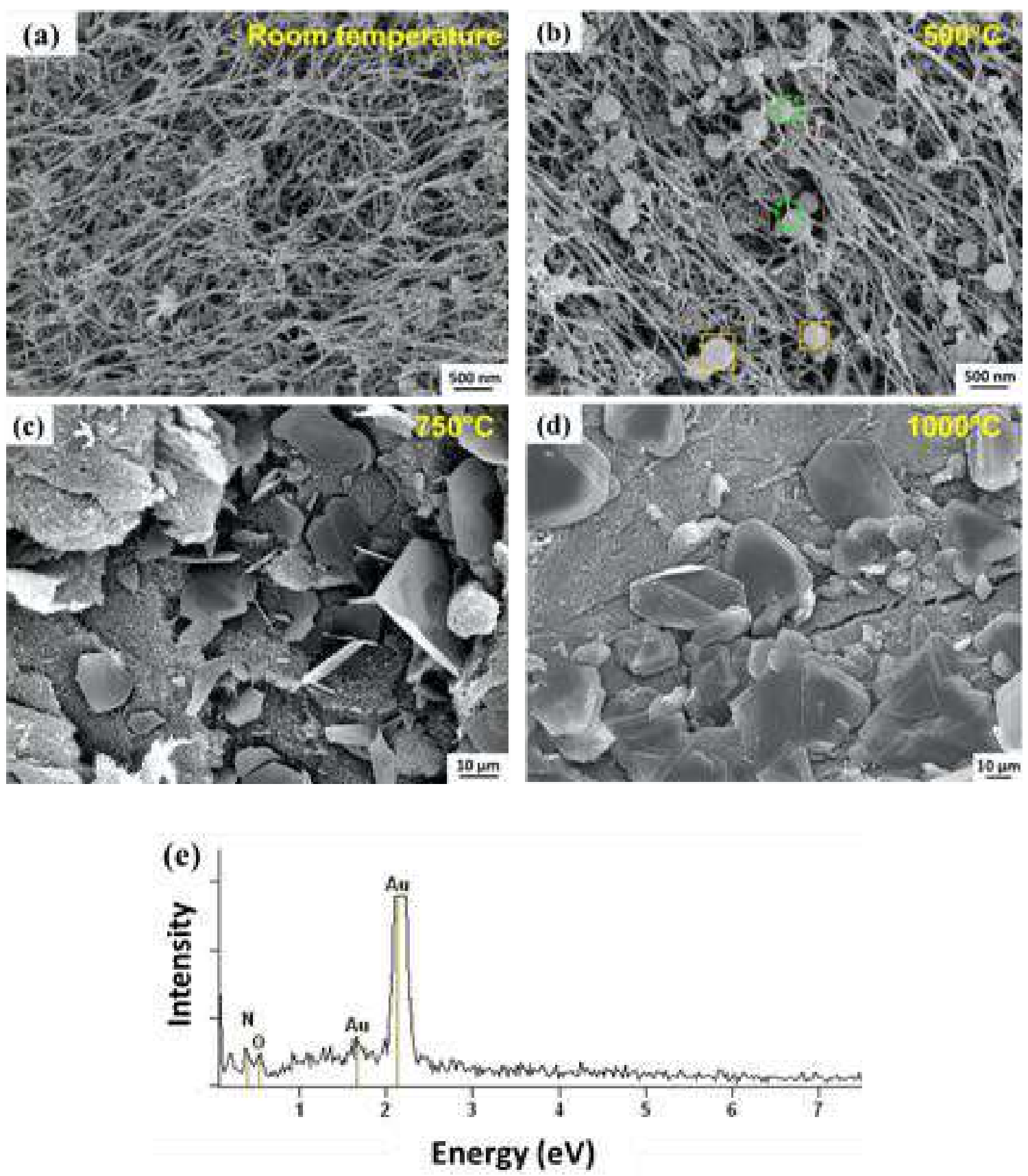

Figure 5.4. SEM images of BNNT sample at different TGA-DSC maximum temperatures showing: (a) pure nanotube cluster at room temperature, (b) growth of nodules in nanotube network at $500^{\circ} \mathrm{C}$, (c) formation of thin stacks of 2-D plates while retaining nanotube clusters at $750^{\circ} \mathrm{C}$, and (d) the formation of dense and thick 2-D stacks of white graphene oxide, with further reduction in the number of nanotube clusters. (e) EDS spectrum of platelets revealed O (Note: Au peaks are due to sputter coating for SEM examination of insulator BNNTs. B and $\mathrm{H}$ cannot be detected by EDS due to their low atomic number, and therefore is not marked here). 
of B-N sheets, have a structure analogous to Graphene Oxide, which also consists of different oxygen-based functional groups attached to parent graphite structure ${ }^{8}$. Therefore, it can be concluded that BNNTs undergo high temperature oxidative unzipping to form Boron Nitride Oxide ${ }^{9}$, a structural analogue of Graphene Oxide (GO). Hence, we term it as 'White Graphene Oxide' (WGO).

\subsubsection{Discussion of Oxidative Unzipping Mechanism}

The oxidation resistance of these ultra-long (up to $\sim 200 \mu \mathrm{m}$ ) and fine nanotubes $(3-6 \mathrm{~nm}$ ) is very high. While the morphological transformation of BNNTs starts at $750^{\circ} \mathrm{C}$, it is seen that even after heating up to $1000^{\circ} \mathrm{C}$, a significant portion of nanotubes remains intact (Figure 5.5a). This suggests that the activation barrier for complete nanotube oxidation is certainly higher than $1000^{\circ} \mathrm{C}$, or else the tubular structures should have completely disintegrated. Local density approximation (LDA) calculations by Zhang et al. for $(3,0)$ BNNT predict the upper limit of this barrier to be as high as $\sim 1500^{\circ} \mathrm{C}^{2}$, hinting to the possibility of the presence of defect sites in the nanotubes which preferentially oxidize at lower temperatures. BNNTs are known to exhibit Stone-Wales (SW) defects ${ }^{10}$, which are characterized by the presence of B-B and N-N homonuclear bonds. In fact, the weak 670 and $1180 \mathrm{~cm}^{-1}$ peaks in IR spectrum of the BNNTs at room temperature are suggestive of the presence of B-B and N-N bonds, respectively (Figure 5.3 ${ }^{11,12}$. These defect sites are reactive due to local strain and bond frustration ${ }^{10}$; therefore the corresponding peaks vanish at higher temperatures. $\mathrm{O}_{2}$ is reported to undergo dissociative chemisorption near these $\mathrm{SW}$ sites. While chemisorption in a pure site is an endothermic process (reported bond energy 


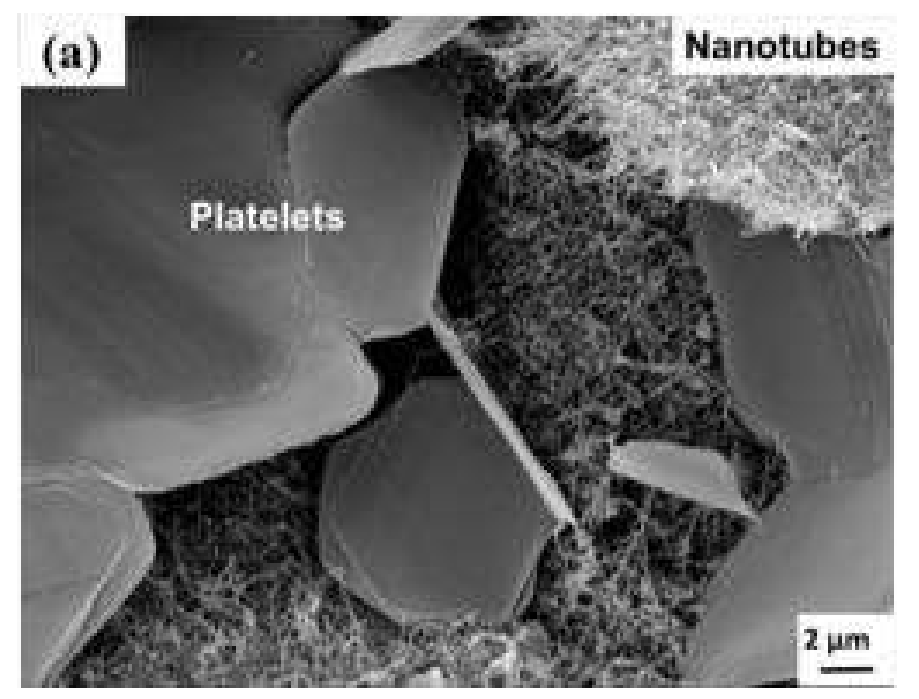

(b)

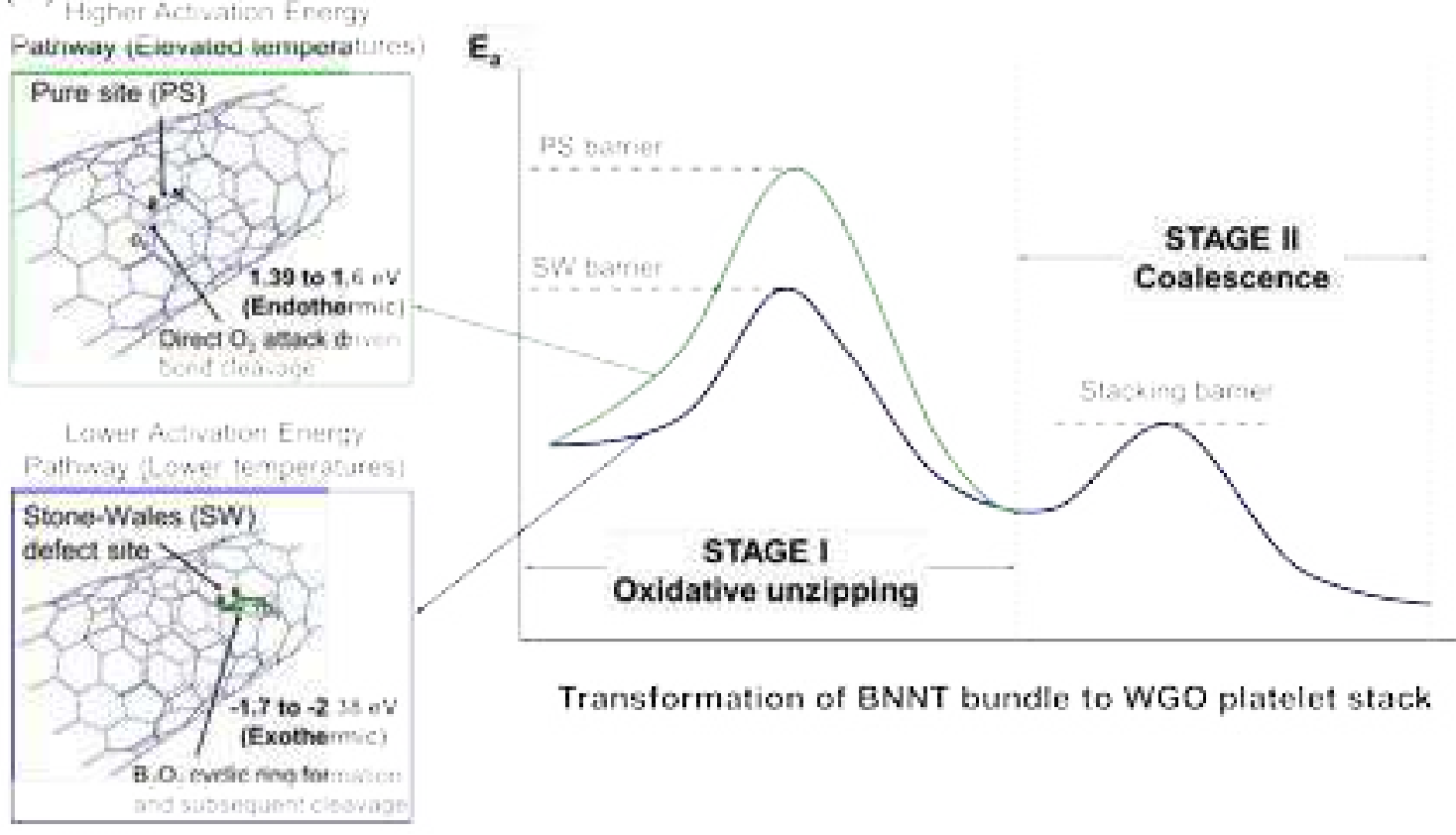

Figure 5.5. (a) High magnification image showing the co-existing nanotube and platelet network, and (b) Schematic diagram showing two-stage BNNT to WGO transformation at elevated temperatures. The activation barrier for the oxidative unwrapping of BNNTs via Stone Wales defect site and pure site is compared.

1.39-1.6 eV), chemisorption of molecular oxygen near Stone Wales defect sites is an exothermic process (reported bond energy $\sim-1.76$ to $-2.38 \mathrm{eV})^{13}$. Therefore, the selective 
oxidation and transformation of nanotubes is possibly related to defects. Chemisorption of molecular $\mathrm{O}_{2}$ at a SW site typically takes place at $\mathrm{B}$ atom, forming a cyclic $\mathrm{B}_{2} \mathrm{O}_{2}$ ring structure $^{13}$, as shown in Figure 5.5b. At higher temperatures, thermal vibration energy is expected to be quite high, causing $\mathrm{B}-\mathrm{B}$ bonds of a $\mathrm{B}_{2} \mathrm{O}_{2}$ ring to cleave resulting in the formation of a strong B-O chemical bond, as also observed in FTIR results. The activation barrier for breaking such a strained 4-member ring is expected to be low (schematically shown in Figure 5.5b). Due to the strain induction, this bond cleavage extends along the entire $\mathrm{BNNT}^{14}$, resulting in the unzipping of the tube to form a planar nanoribbon. Contrary to this, pure sites have a tendency for physisorption instead of chemisorption due to the mismatch of atomic orbitals of $\mathrm{O}_{2}$ with that of $\mathrm{BNNT}^{13}$. Chemisorption, therefore, is likely to take place at a pure site only under drastic conditions, such as high temperatures. Complete disintegration of tubular structures will take place only after the barrier for B-O chemical bond formation/ B-N bond dissociation is exceeded, which appears to be higher than $1000^{\circ} \mathrm{C}$ for the nanotubes studied here (Figure 5.5b).

\subsubsection{Discussion of White Graphene Oxide Formation Mechanism}

The mechanism of formation of white graphene oxide (WGO) platelets can be explained in two major steps: (i) high-temperature oxidative unzipping of BNNTs, and (ii) coalescence of the unfolded nanoribbons to form a continuous network of 2D plates (schematically shown in Figure 5.5). This can be seen in the SEM image of the BNNT sample subjected to TGA-DSC heating up to $750^{\circ} \mathrm{C}$ (Figure 5.6a). The regions encircled in yellow are unreacted BNNTs, retaining their fine tubular structure. Regions encircled in

orange consist of nanotubes unwrapped to form planar ribbons, which are in the process of 
coalescing together to form 2D plates. Regions marked in green show fully formed, integrated and continuous 2D plates of WGO.
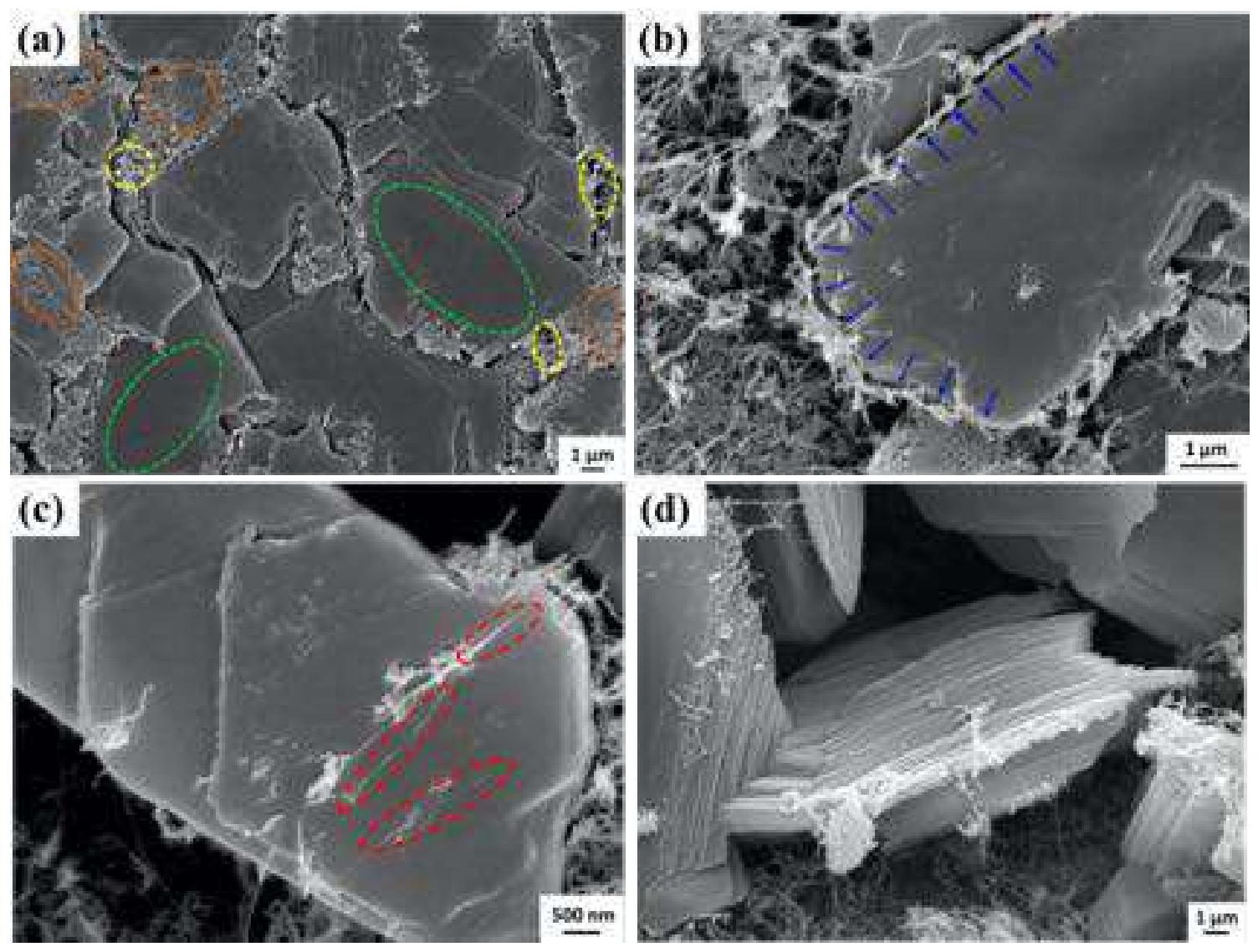

Figure 5.6. SEM images showing the progression of the oxidative transformation of BNNT: (a) unreacted, partially uncurled and completely transformed regions marked yellow, (b) sideways growth of 2D WGO platelet by unzipping and coalescence of BNNTs, (c) uncurled nanoribbon forming a new peel over the previous layer in WGO stack, and (d) fully formed thick stack of WGO layers, with some dangling nanotubes visible along the sideways.

The WGO platelets grow with time and temperature. These 2D plates grow by sideways expansion as well as the stacking of new planar layers over the existing ones. The mechanism of sideway growth of these 2D plates is elucidated in Figure 5.6b, where the transformed plates are surrounded by unreacted nanotubes. These BNNTs will unzip and 
merge with the parent plate, resulting in the growth of a continuous array of planar WGO layers (shown with blue arrows). Figure 5.6c shows the unzipped and partially cleaved BNNTs in the process of forming a new peel over the previous layer. The tubular/ semitubular structures encircled in red represent the positions where nanotubes unwrap and start forming an ultra-thin 2D layer at the top, forming stacks of WGO. Figures 5.6a-c correspond to a BNNT sample after $750^{\circ} \mathrm{C}$ TGA-DSC heating. A fully formed, thick stack of WGO layers at $1000^{\circ} \mathrm{C}$ is shown in Figure 5.6d. This is demonstrative of multi-layered architecture consisting of 2D plates formed by high-temperature BNNT unzipping and coalescence. Some of the untransformed nanotubes can be seen dangling from the stack edges.

\subsection{High-Temperature Mechanical Properties of Boron Nitride Nanotube}

\subsubsection{Local Indentation Compression}

The buckypaper was subjected to compression by a flat-ended diamond probe of $10 \mu \mathrm{m}$ diameter. These tests were performed inside the scanning electron microscope (SEM) for real-time visualization of load transfer in the nanotube assembly (Figure 5.7a). The indentation tests were performed at room temperature, $250^{\circ} \mathrm{C}$ and $500^{\circ} \mathrm{C}$. Loaddisplacement curves reveal an elasto-plastic deformation behavior. An increase in deformation is noticed as the temperature increases (Figure 5.7b). The difference between deformation at room temperature and $250^{\circ} \mathrm{C}$ is very marginal (around $8 \mu \mathrm{m}$ ); but there is a visible enhancement of total penetration depth at $500^{\circ} \mathrm{C}(\sim 12 \mu \mathrm{m})$. However, the final residual plastic deformation is not too high even at $500^{\circ} \mathrm{C}(\sim 6.2 \mu \mathrm{m})$, as compared to room 
temperature indentation $(\sim 5.7 \mu \mathrm{m})$. This suggests the activation of additional elastic mechanisms with increasing temperature. The Supplementary Videos (V5.1-V5.3) show the real-time SEM video of the indentation for the tests performed at room temperature, $250^{\circ} \mathrm{C}$ and $500^{\circ} \mathrm{C}$, respectively. The buckypaper exhibits a trampoline-like behavior. The compression loading visibly stretches the buckypaper in the vicinity of the indenter probe up to radial distances $>10 \mu \mathrm{m}$ (Figure 5.7a), indicating effective load transfer/ dispersion due to the interconnected nanotube network. During compression, the stress-strain relationship is expressed as volumetric change ${ }^{15}$ :

$$
\frac{\Delta V}{V_{0}}=-C_{b} \Delta P
$$

where $V_{0}$ is the initial volume, $\Delta V$ is the volume change due to compression, $\Delta P$ is the pressure change or the stress applied during indentation and $C_{b}$ is the compressibility of the buckypaper. Since the localized indentation deformation is largely one-dimensional in nature, the volume change in Eq (5.1) can be expressed as a change in thickness $(\Delta t)$ during $\operatorname{compression}^{15}$ :

$$
\frac{\Delta t}{t_{0}}=-C_{b} \Delta P
$$

where $t_{0}$ is the initial thickness of the buckypaper, which is $80 \mu \mathrm{m}$. Change in thickness $(\Delta t)$ is equal to the indenter displacement $(d)$ and change in pressure $(\Delta \mathrm{P})$ is the indentation stress, which can be computed as $F / A_{p}$, where $\mathrm{F}$ is indentation force and $A_{p}=\pi r^{2}$, is the area of indenter probe compressing the material. The peak displacements at $5 \mathrm{mN}$ applied force (at room temperature, $250^{\circ} \mathrm{C}$ and $500^{\circ} \mathrm{C}$ ) are plotted in Figure 5.7b. Substituting these values to Eq. (5.2), the compressibility of BNNT buckypaper can be determined as a 
(a) INITIAL CONFIGURATION

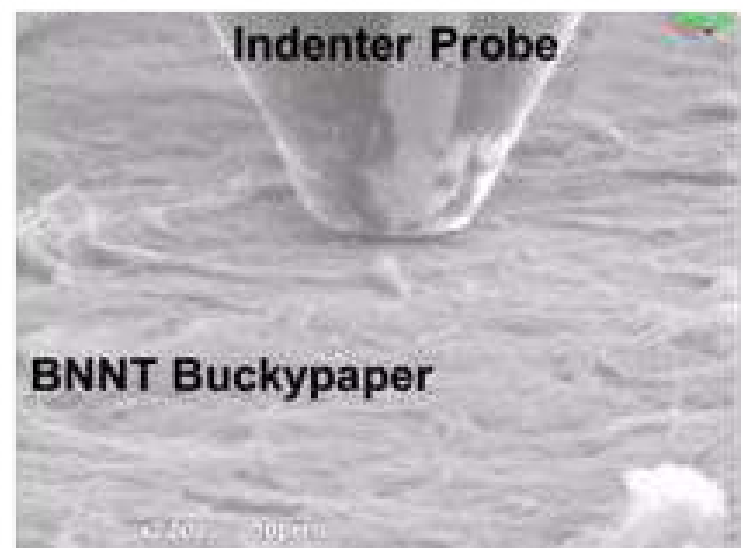

AT PEAK APPLIED LOAD

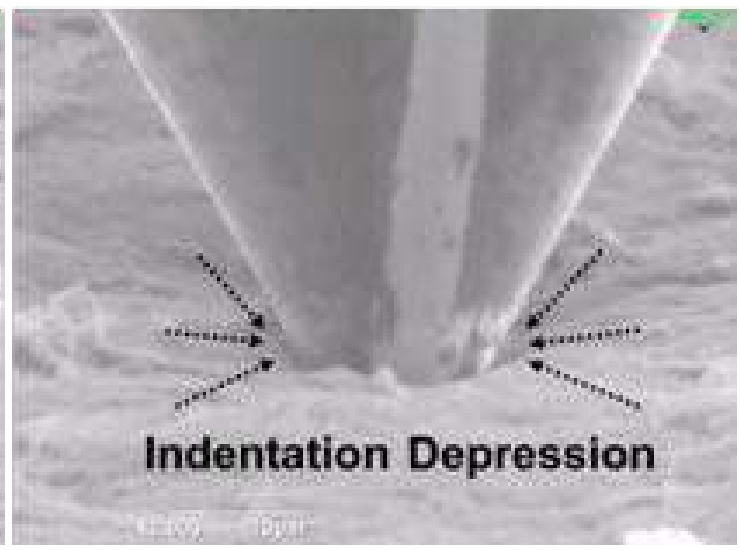

(b)

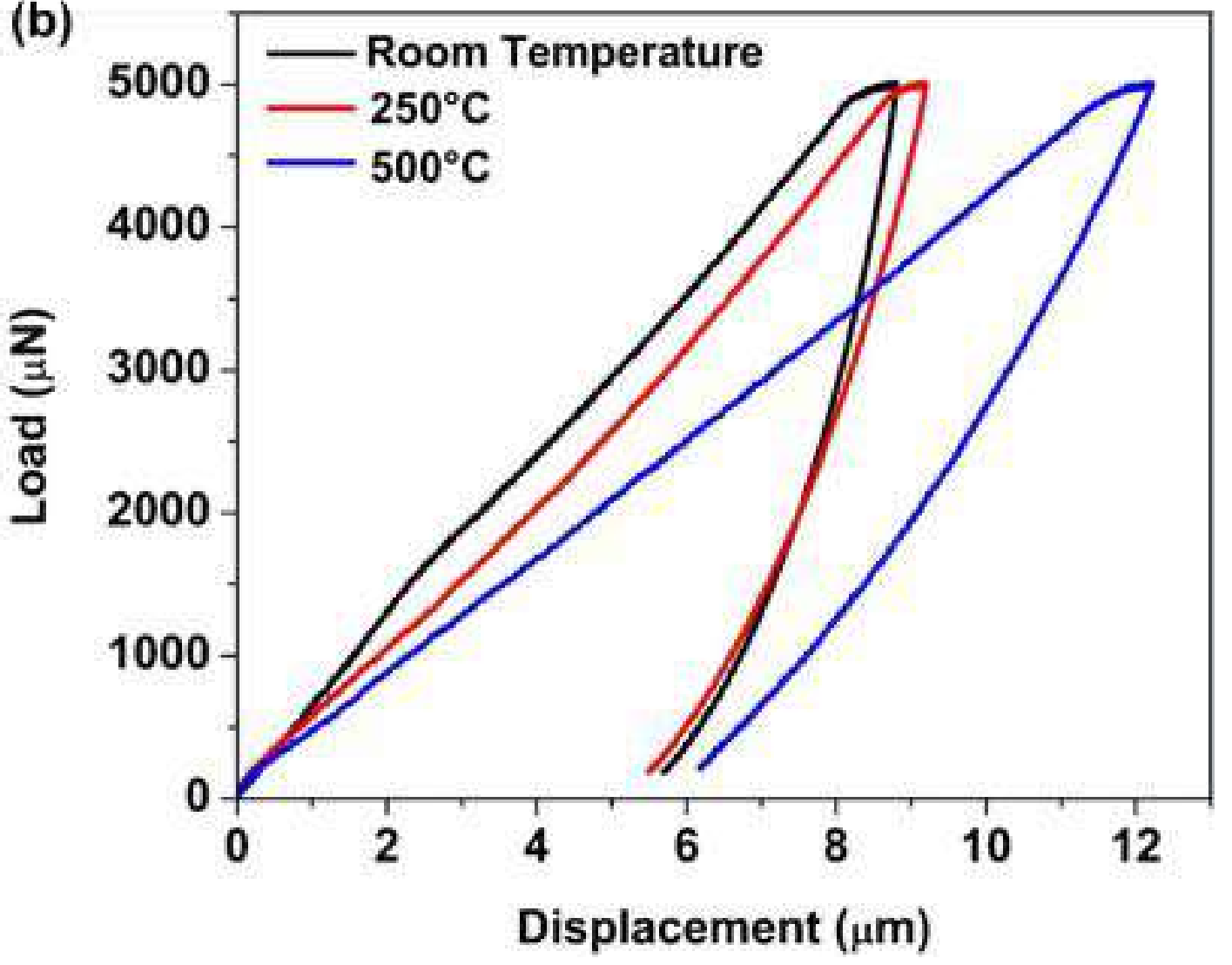




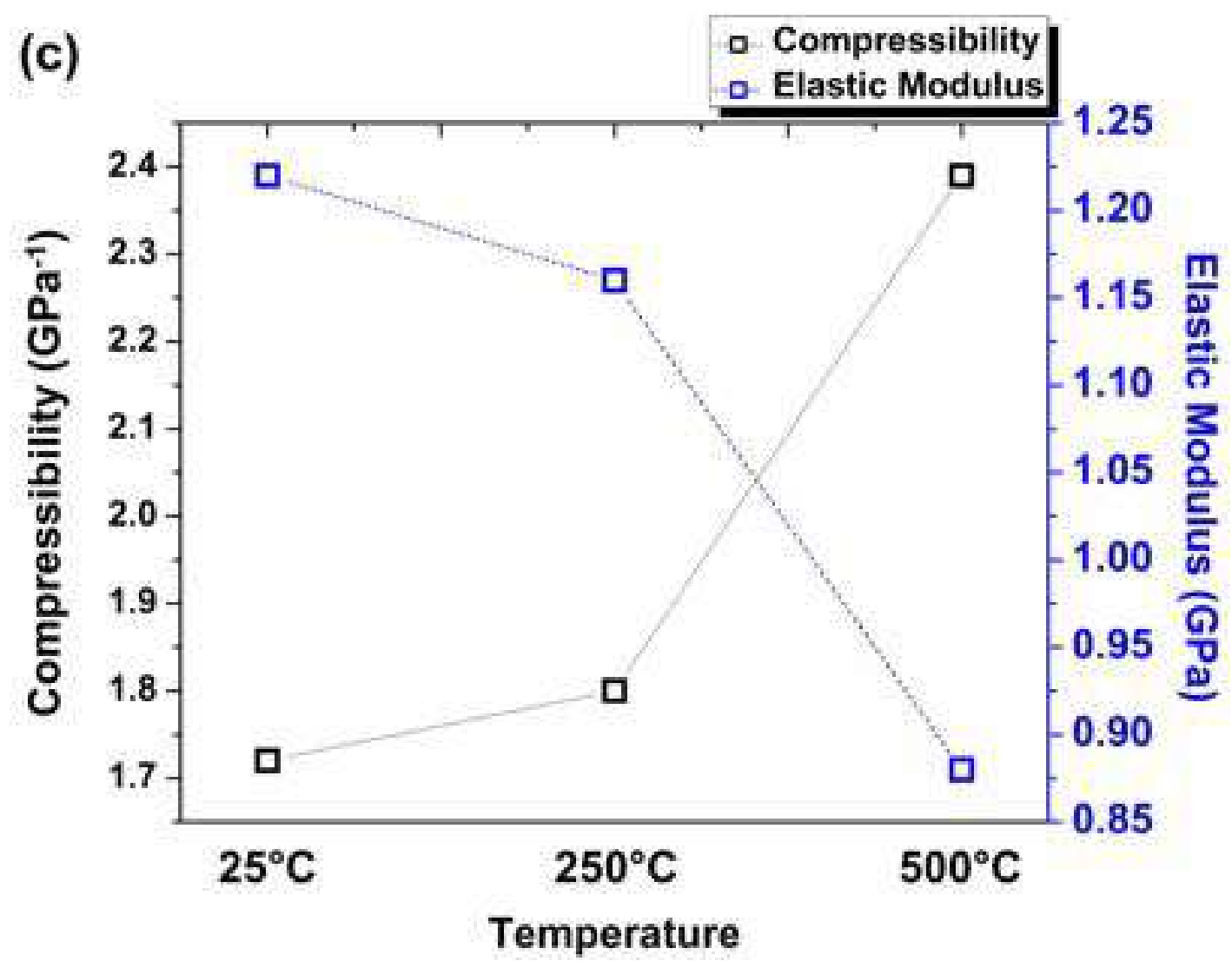

(d) BNNT Kinking Mechanism

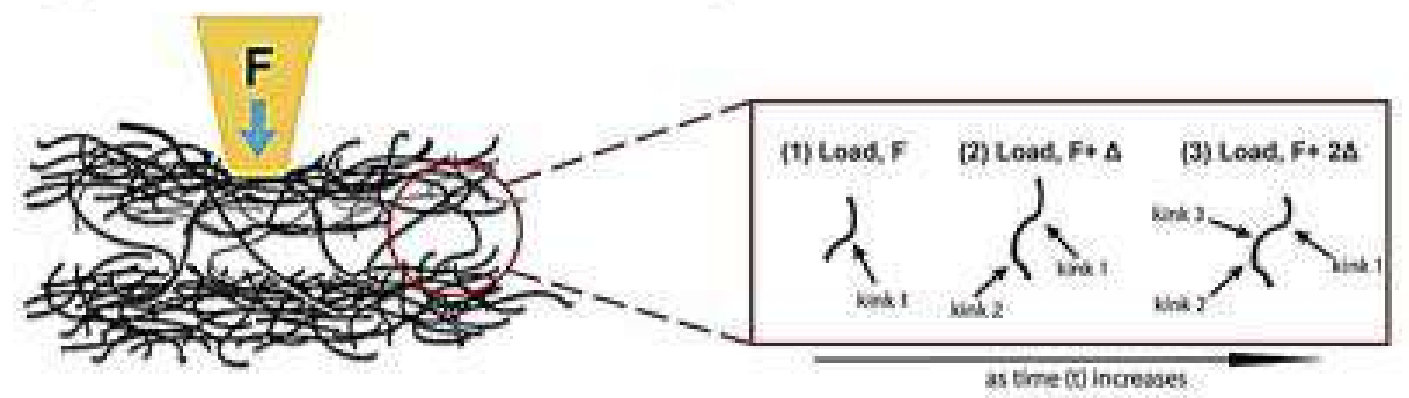

Figure 5.7. (a) In-situ nanoindentation of BNNT buckypaper inside SEM chamber: indentation compression leads to elastic stretching of buckypaper due to highly interconnected network, (b) nanoindentation load-displacement response of buckypaper at room temperature, $250^{\circ} \mathrm{C}$ and $500^{\circ} \mathrm{C}$ temperatures, (c) local compressibility and elastic modulus of buckypaper determined during nanoindentation as a function of temperature, and (d) schematic representation of BNNT buckling/ kinking mechanism active during indentation compression. The real-time SEM videos are provided as supplementary videos V5.1-V5.3. 
function of temperature. The localized compressibility of the buckypaper increases from $1.7 \mathrm{GPa}^{-1}$ at room temperature to $\sim 2.4 \mathrm{GPa}^{-1}$ at $500^{\circ} \mathrm{C}$ (Figure 5.7c). The elastic modulus of the buckypaper as a function of temperature is computed from the compressibility values, based on the following relation:

$$
E=\frac{3(1-2 v)}{C_{b}}
$$

where $v$ is the Poisson's ratio, which can be assumed to be $\sim 0.15$ for the buckypaper ${ }^{16}$. Based on Eq. (5.3), the elastic modulus of BNNT buckypaper is computed to be $\sim 1.2 \mathrm{GPa}$ at room temperature but drops to $0.8 \mathrm{GPa}$ at $500^{\circ} \mathrm{C}$ (Figure 5.7c).

Upon compression, the nanotube pillars that interconnect the multiple layers of buckypaper will undergo buckling. BNNT is highly flexible, and elastic buckling of nanotube happens by kink formation, schematically shown in Figure 5.7d ${ }^{17}$. As the applied load increases, each nanotube can undergo multiple kinking phenomena, shown in a previous report ${ }^{17}$. During kink formation, the lattice structure of BNNT is distorted, creating dislocation-like defects and missing atomic planes. Golberg and co-workers reported a spring-like recovery of kinks upon removal of mechanical load ${ }^{17}$. Because of their reversible character, they are also referred to as "momentary kinks". However, for the bending angles greater than $115^{\circ}$, the nanotubes tend to retain a residual buckle, responsible for permanent deformation ${ }^{17}$. This explains the elasto-plastic load-displacement behavior observed in Figure 5.7. It is known that BNNTs are often characterized by the presence of vacancies, particularly if the growth process is far from thermodynamic equilibrium ${ }^{18,19}$. Thermal vacancy migration is one of the possible mechanisms for reorganization and 
agglomeration of the vacancy defects to form dislocation lines. The activation barrier for the migration event is reported to be $3-6 \mathrm{eV}^{18}$, making the formation of dislocation line at room temperature thermodynamically infeasible. However, vacancy migration would be favored at elevated temperatures, giving rise to the formation of extended dislocation defects in BNNT. The presence of dislocations is likely to enhance the deformation experienced by the nanotubes due to dislocation motion. This was evident from relatively higher penetration depths noticed during high temperature nanoindentation in comparison to the room temperature indentation depths (Figure 5.7b). In addition to the intrinsic vacancy effects, compression of the buckypaper structure will also induce inter-tube sliding. Energy is consumed to overcome van der Waals attraction between the tubes for sliding to happen. The inter-tube mechanisms are likely to play a more important role during bulk-scale and high-load testing, and are discussed in a later section (Section 5.2.3) on macro-scale tensile testing.

\subsubsection{Cyclic Indentation Response}

While the free-standing buckypaper displays stable elastic properties at elevated temperatures, the ability to retain structural integrity and mechanical properties over repeated deformation cycles is vital for real-world applications. The buckypaper was subjected to cyclic loading-unloading-reloading quasi-static compression by in-situ nanoindentation, to examine the deformation characteristics after prolonged local loading. The load-displacement curves demonstrate viscous effects, evident from the formation of hysteresis loops during cyclic loading (Figure 5.8a). The cyclic indentation was performed 
for 50 cycles. The Supplementary Videos (V5.4-V5.6) show real time deformation inside SEM, for cyclic tests performed at room temperature, $250^{\circ} \mathrm{C}$ and $500^{\circ} \mathrm{C}$, respectively. The videos reveal the compression-recovery sequence as the indenter is loaded and unloaded. As noted previously, the buckling of nanotube pillars interconnecting the buckypaper layers is reversible in nature ${ }^{17}$. The formation and annihilation of kinks provide this high degree of reversible cyclic deformation, making the free-standing BNNT structure damage tolerant. Nevertheless, some residual plastic strain is also noticed, due to a certain degree of permanent buckle (when the nanotubes deform excessively, typically $>115^{\circ}$ angle ${ }^{17}$ ). As a result, some of the energy imparted by the indenter is dissipated, resulting in the hysteresis loop seen in Figure 5.8a.

It is noteworthy that van der Waals forces of attraction between the nanotubes hold together the BNNT assembly. There are van der Waals interactions between individual nanotubes, as well as the multiple layers constituting the buckypaper. The van der Waals forces acting between multiple layers can be approximated by the equation for inter-plane interactions $^{20}$ :

$$
F_{V D W}=\frac{A}{6 \pi h^{3}}
$$

where $F_{V D W}$ is the van der Waals force between the plane sheets, $A$ is the Hamaker's coefficient and $h$ is the inter-planar separation. Since BNNT layers are porous, this equation can be modified to account for the porosity:

$$
F_{V D W}=\frac{(1-p) A}{6 \pi h^{3}}
$$


where $p$ is the porosity, which is 0.8 for the buckypaper investigated in this study ${ }^{21}$. The energy change during cyclic compression can be modeled as:

$$
E=\frac{d F_{V D W}}{d h}=\frac{A}{30 \pi h^{4}}
$$

Based on Eq. (5.6), the energy changes inversely to the fourth power of inter-layer separation, h. As noted before, the compressibility of the buckypaper assembly increases with the increase in test temperature (Figure 5.7c). As a result, the separation ' $h$ ' between the layers will decrease, resulting in enhanced viscous effects/ energy dissipation (based on Eq. 6). This is evident from the nature of F-h hysteresis loops shown in Figure 5.8a, with more prominent hysteresis loops at $500^{\circ} \mathrm{C}$. In addition to inter-layer van der Waals interactions, inter-tube sliding will be active during the mechanical loading of the buckypaper. The interfacial sliding may involve stick-slip events, which can lead to energy dissipation $^{22}$. The ability to dissipate energy is promising for superior impact tolerance.

It is seen from Supplementary Videos (V5.4-V5.6) that the indentation loading/ unloading not only compresses the buckypaper along the z-axis, but also causes elastic stretching and recovery around the indenter. The interconnected and entangled nanotubes in the buckypaper act as a network of springs, with the tendency to revert to the original configuration upon unloading. Digital image correlation (DIC) analysis of the real-time snapshots was performed to obtain microstructure strain contours. In DIC analysis, the realtime snapshots of the microstructure (during deformation) are compared/ analyzed with respect to the starting snapshot, to capture the changes in specimen surface incrementally as the force is applied ${ }^{23}$. The software tracks and correlates the pixels in the deformed snapshots with respect to the starting image. This relative comparison aids in determining 
the full-field strain map, showing the spatial distribution of strains in the microstructure as a function of mechanical loading ${ }^{24,25}$. Figure $\mathbf{5 . 8 b}$ shows the strain maps for room temperature indentation, clearly showing the long-distance stress transfer, evident from the development of strains even $\sim 50 \mu \mathrm{m}$ away from the local indentation. The ability to disperse the force provides the buckypaper assembly high degree of trampoline-like elasticity (Videos V5.1-V5.6) and prevents local failure/ rupture of the BNNT assembly even for local indentation-induced stresses as high as $\sim 60 \mathrm{MPa}$. The DIC maps with colored contours indicate a variable stress-state, which is highly dependent on the local disentanglement and re-entanglement phenomena. The map clearly shows that the microstructure strains are pronounced at peak loading state as the local depression caused by the indenter probe is transmitted as elastic stretching in the vicinity. During the first loading cycle, the local strains are obtained to be as high as $\sim 3 \%$ when $5 \mathrm{mN}$ load is applied. It is also noted that even after complete unloading, there are residual strains in the buckypaper $(|\varepsilon|<1 \%)$ which signifies some degree of 'unrecovered' permanent deformation. This residual strain progressively builds up after each cycle. The final residual strain at the end of the $50^{\text {th }}$ cycle was observed to be higher than $1 \%$ (Figure $\mathbf{5 . 8 b}$ ). The permanent deformation of the buckypaper can be related to the topological transformation of the nanotube network during indentation-induced stretching. As observed in Figure 4.3, the buckypaper microstructure comprises of nodes where the nanotubes intersect. During the stretching and release of the buckypaper (due to cyclic loading-unloading), the nanotubes are likely to undergo detachment-attachment phenomena at these nodal junctions ${ }^{26}$. Upon unloading, the nanotubes do not necessarily reattach with the same partner; many of the nanotubes tend to pair with new partners to form the nodal junctions. 


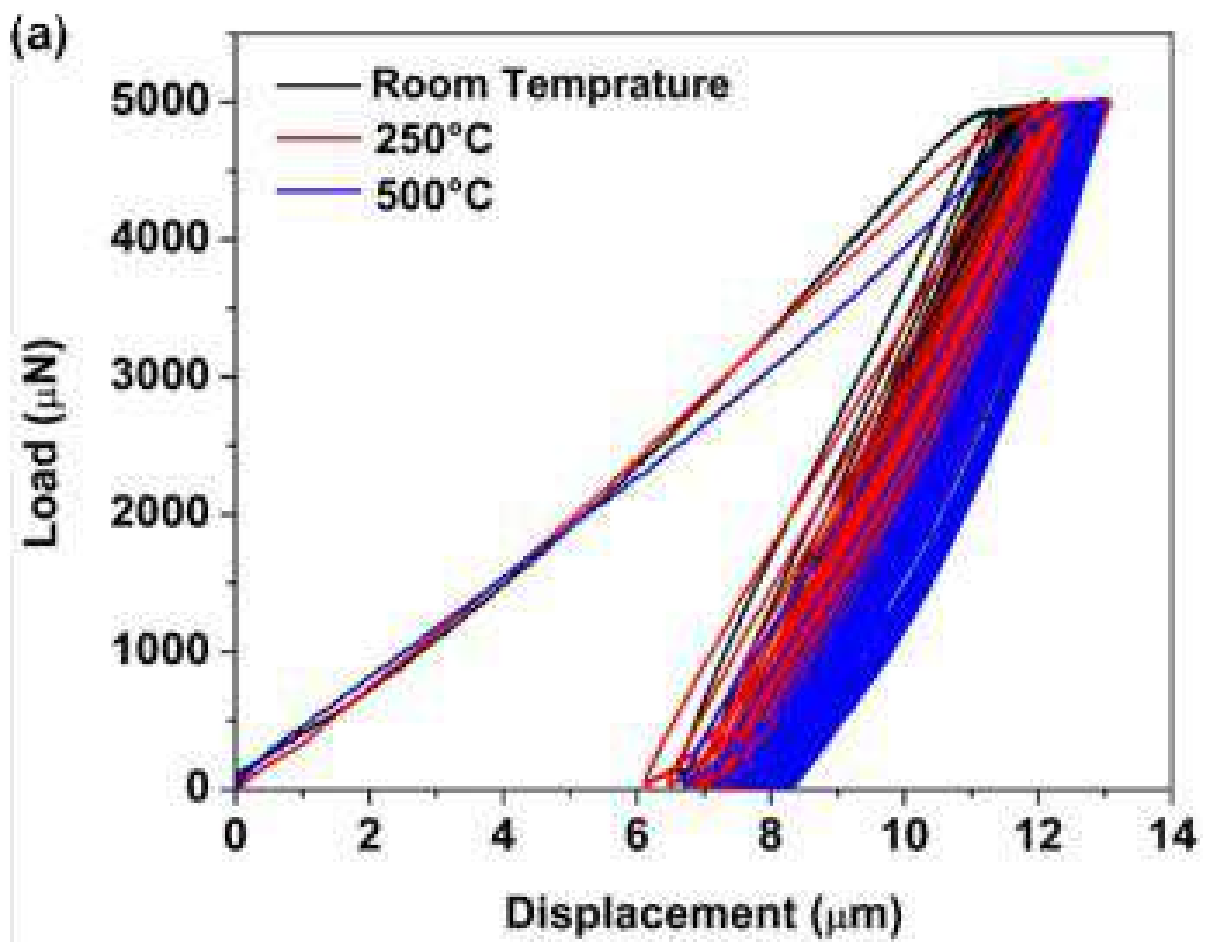

(b)

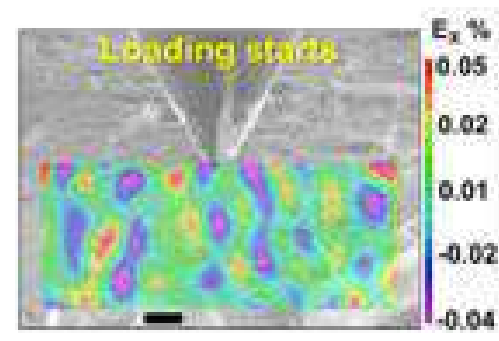

Cycle 1
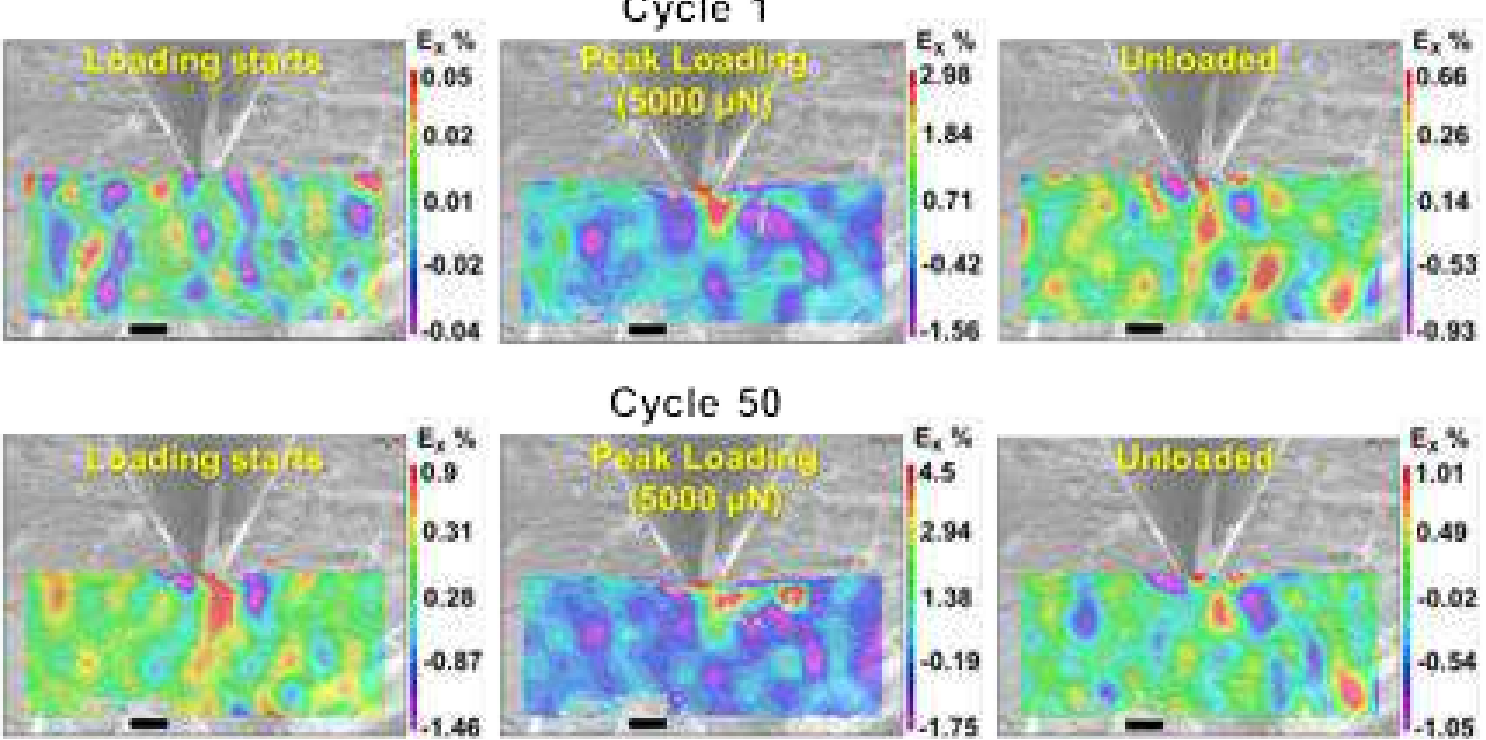

Cycle 50

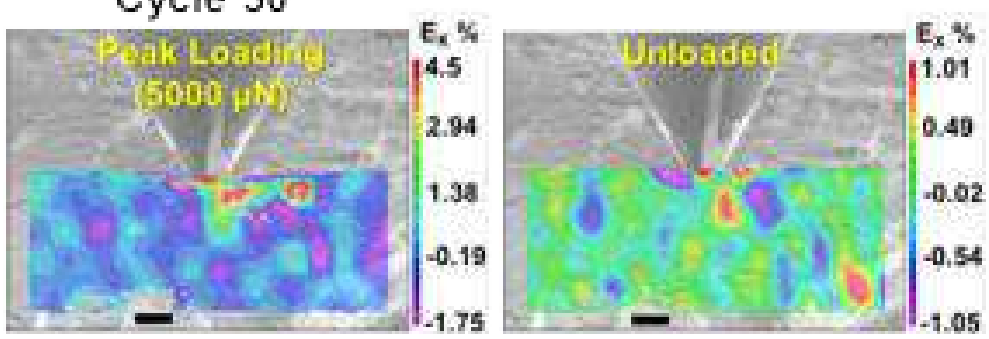



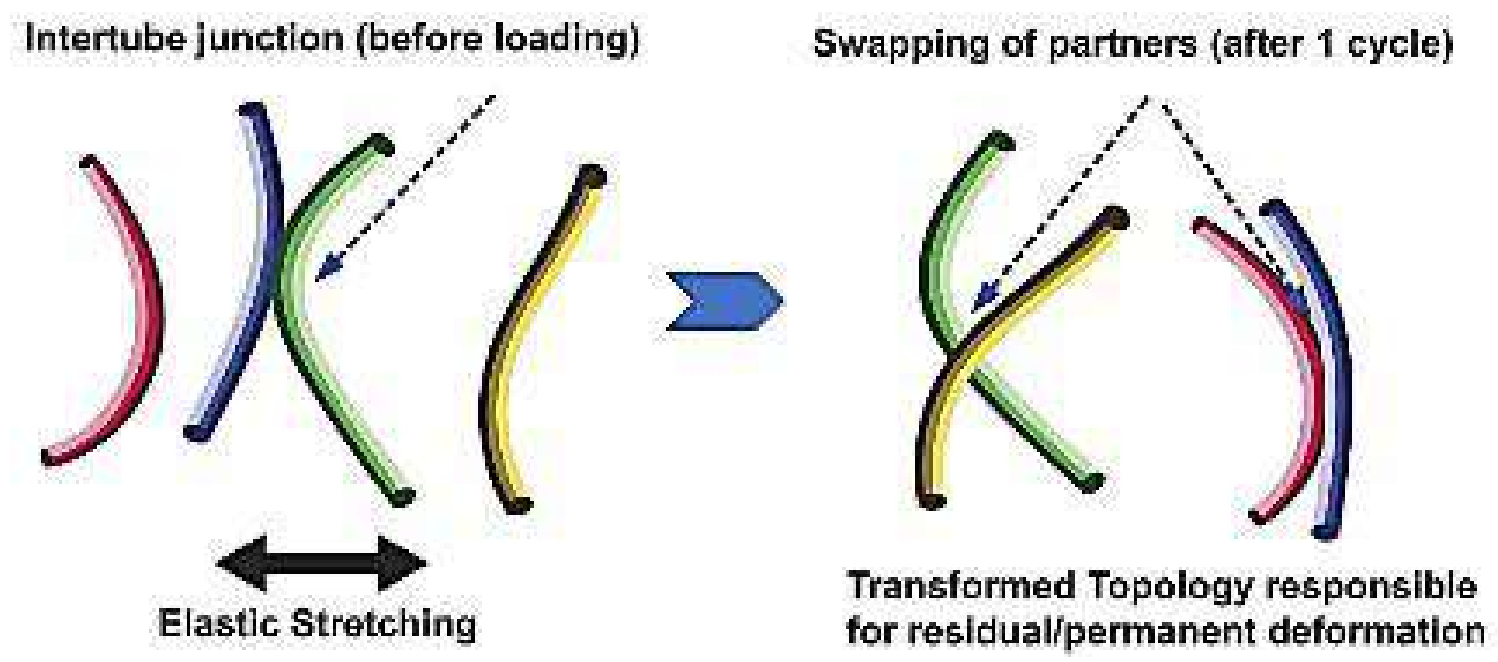

Figure 5.8. (a) Nanoindentation load-displacement response for cyclic loading-unloading (50 cycles) with hysteresis loops at room temperature, $250^{\circ} \mathrm{C}$ and $500^{\circ} \mathrm{C}$, (b) microstructure strain contours developed in the buckypaper during indentation loading for $1^{\text {st }}$ and $50^{\text {th }}$ cycles are shown, attesting long-distance stress-transfer and accumulation of residual strain over the 50 deformation cycles (scale bar $20 \mu \mathrm{m}$ ), and (c) schematic representation of the detachment-attachment mechanism at nanotube junctions leading to topological transformation due to stretching of buckypaper (during cyclic indentation). The real time SEM videos are provided as supplementary videos V5.4-V5.6.

This leads to topological transformation, resulting in some degree of residual permanent deformation observed in Figure 5.8a and 5.8b. This detachment-attachment mechanism active during the stretching of buckypaper is illustrated in Figure 5.8c.

It is noteworthy that the nanoindentation modulus values $(0.8-1.2 \mathrm{GPa})$ are much higher than the 'tensile' elastic modulus reported in the literature for BNNT buckypaper $(\sim 10-20 \mathrm{MPa})^{21}$, primarily because nanotube disentanglement is likely to be more prominent in tension resulting in compromised stiffness. This difference in tensioncompression mechanics would be vital when engineering BNNT-based composites. Hence, 
the next section explores the tensile properties of the buckypaper as a function of temperature.

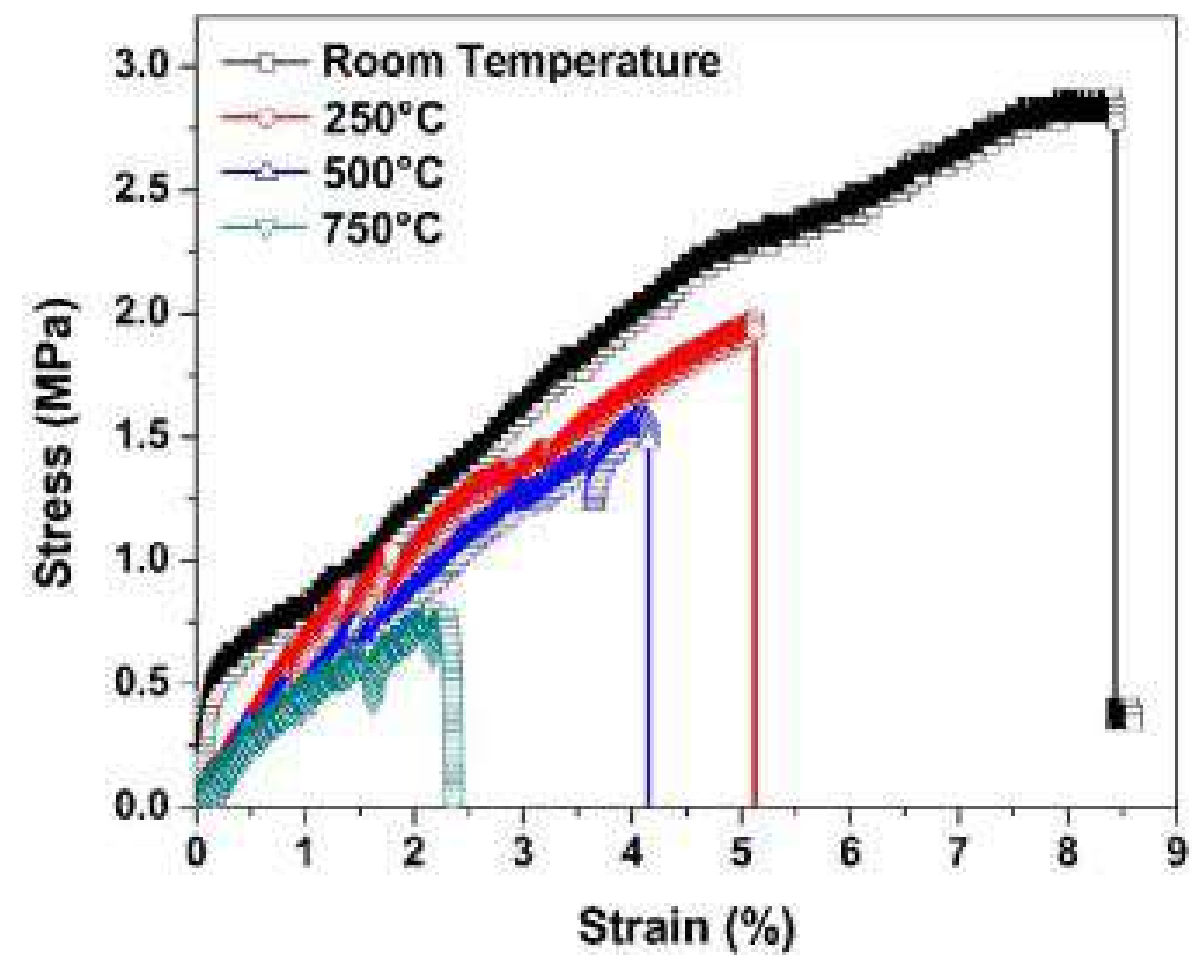

Figure 5.9. Tensile stress-strain curves for BNNT buckypaper deformed at room temperature, $250^{\circ} \mathrm{C}, 500^{\circ} \mathrm{C}$ and $750^{\circ} \mathrm{C}$.

\subsubsection{High Temperature Tensile Deformation}

To examine the bulk structural scale mechanical properties, the buckypaper was subjected to tensile loading and the stress-strain characteristics were obtained over a range of temperature, i.e. at room temperature, $250^{\circ} \mathrm{C}, 500^{\circ} \mathrm{C}$, and $750^{\circ} \mathrm{C}$ (Figure 5.9). The buckypaper was characterized by an ultimate tensile strength (UTS) of $\sim 2.8 \mathrm{MPa}$ at room temperature. However, it should be noted that with $80 \%$ porosity, the 'true' cross-section 
area that bears tensile load is much lower: $A_{\text {true }} \sim(1-p) \times A_{\text {apparent }}$, where $\mathrm{p}$ is porosity and $A_{\text {Appparent }}$ is the apparent cross-section area determined by physically measuring the width and thickness of the tensile specimens. When normalized by taking porosity into consideration, the 'true' tensile strength at room temperature is computed to be $~ 13.8 \mathrm{MPa}$. A progressive drop in strength was observed with increasing temperature. The true strength was found to be $\sim 9.8,7.9$ and $3.8 \mathrm{MPa}$ at 250,500 and $750^{\circ} \mathrm{C}$, respectively. The strain at failure is $\sim 8.4 \%$ at room temperature, but it drops to $\sim 2.4 \%$ at $750^{\circ} \mathrm{C}$.

To develop insight into failure mechanisms in real-time, the tensile deformation of buckypaper at room temperature was captured by a high-speed camera. The sequence of events during tensile loading is summarized in Figure 5.10. The real-time snapshots show that the crack initiates at the location of stress-concentration, which is a pre-crack tip in this case (Snapshots \# 1 and 2). The crack propagation is characterized by extensive deflections (Snapshot \# 3). The crack advances in the buckypaper via disentanglement and detachment of BNNTs held together by van der Waals forces. Since the nanotubes in buckypaper microstructure are clustered, the disentanglement takes place locally in the microstructure, at the sites of least resistance to detachment or sites with poor inter-tube entanglement. The 3D network of nanotubes delays crack propagation due to local disruptions in the crack pathway. This was evident from the zig-zag crack pathway observed in Figure 5.10. The high-speed camera video (Supplementary Video V5.7) shows the crack propagation takes place in multiple steps, with intervening pauses or halts. This is attributed to extensive crack bridging by BNNTs. The pulled-out nanotubes acting as 
crack bridges are encircled in the Snapshots \# 4. These bridges were found to be as long as 90-100 $\mu \mathrm{m}$, and enhance the resistance to failure. For crack to propagate, the applied mechanical energy is absorbed for breaking the nanotube bridges. This results in delayed crack advancement and failure, as observed in Supplementary Video 5.7, with multiple halts during crack propagation. The final snapshot (Snapshot \# 6) after failure shows a zigzag tearing of the buckypaper.
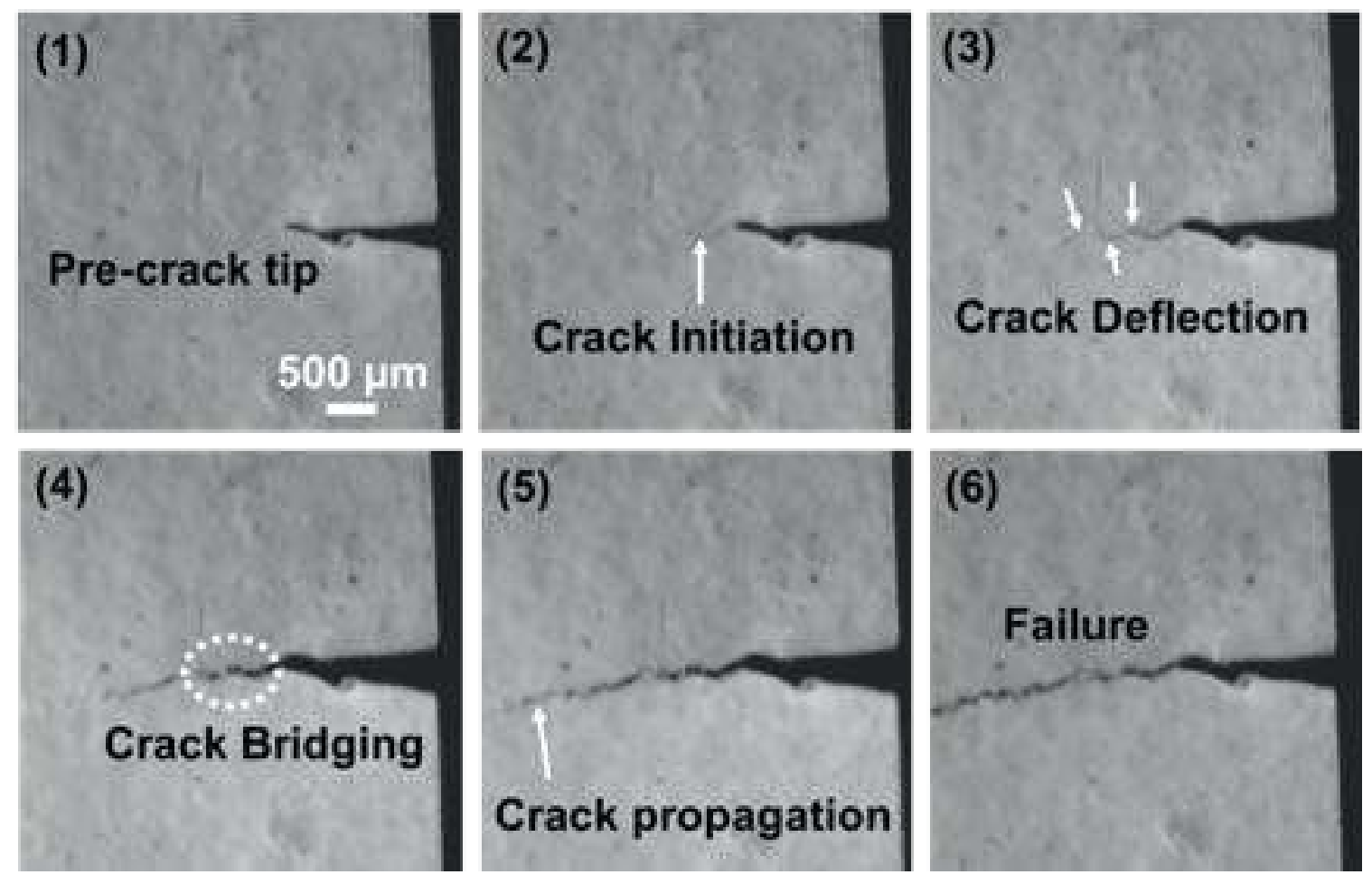

Figure 5.10. Sequence of deformation events recorded by high speed camera at 5,000 frames per second during tensile testing of BNNT buckypaper at room temperature.

The deformation mechanisms at different temperatures were examined by postfailure electron microscopy of buckypaper specimens after the samples were cooled to the room temperature (Figure 5.11). The failed cross-section reveals the delamination/ separation of layers constituting the buckypaper (Figure 5.11a). The extent of detachment 
is more prominent at elevated temperatures. For tensile failure at $750^{\circ} \mathrm{C}$, it can be seen that the nanotube pillars are pulled out. The thermal transformation of BNNT at elevated temperatures is responsible for poor inter-layer cohesion. SEM micrographs in Figure 5.11b reveal the transformation and coalescence of BNNTs to form platelet-like structures at 500 and $750^{\circ} \mathrm{C}$. High temperature exposure results in the diffusive bonding of the unzipped nanoribbons. This will result in poor interconnectivity of the nanotube network, leading to deteriorated failure resistance. It was discussed in Section 5.5 that defect sites undergo oxidative transformations due to the lower activation barrier for oxidation. This results in certain regions in the microstructure having a discontinuous/ disrupted nanotube network, responsible for poor load-bearing ability at $750^{\circ} \mathrm{C}$ (Figure 5.9). During tensile deformation, the buckypaper microstructure undergoes reorganization, such that the tubes are aligned along the loading direction. This aids in enhanced load-bearing ability as nanotubes display superior mechanical properties along their axial length. The realignment phenomenon is shown in the SEM micrographs in Figure 5.11c. The images show nanotube bundles pulled out due to tension. The re-alignment behavior was noticed for each individual layer of the buckypaper assembly. However, the pull-out is not as prominent at $750^{\circ} \mathrm{C}$. The thermal transformation results in deterioration of the nanotube network. This was observed upon close examination of the failed cross-section (Figure 5.11d). Coalescence of BNNTs at high temperatures results in the arrested ability for nanotube reorientation. 
(a) Delamination of layers

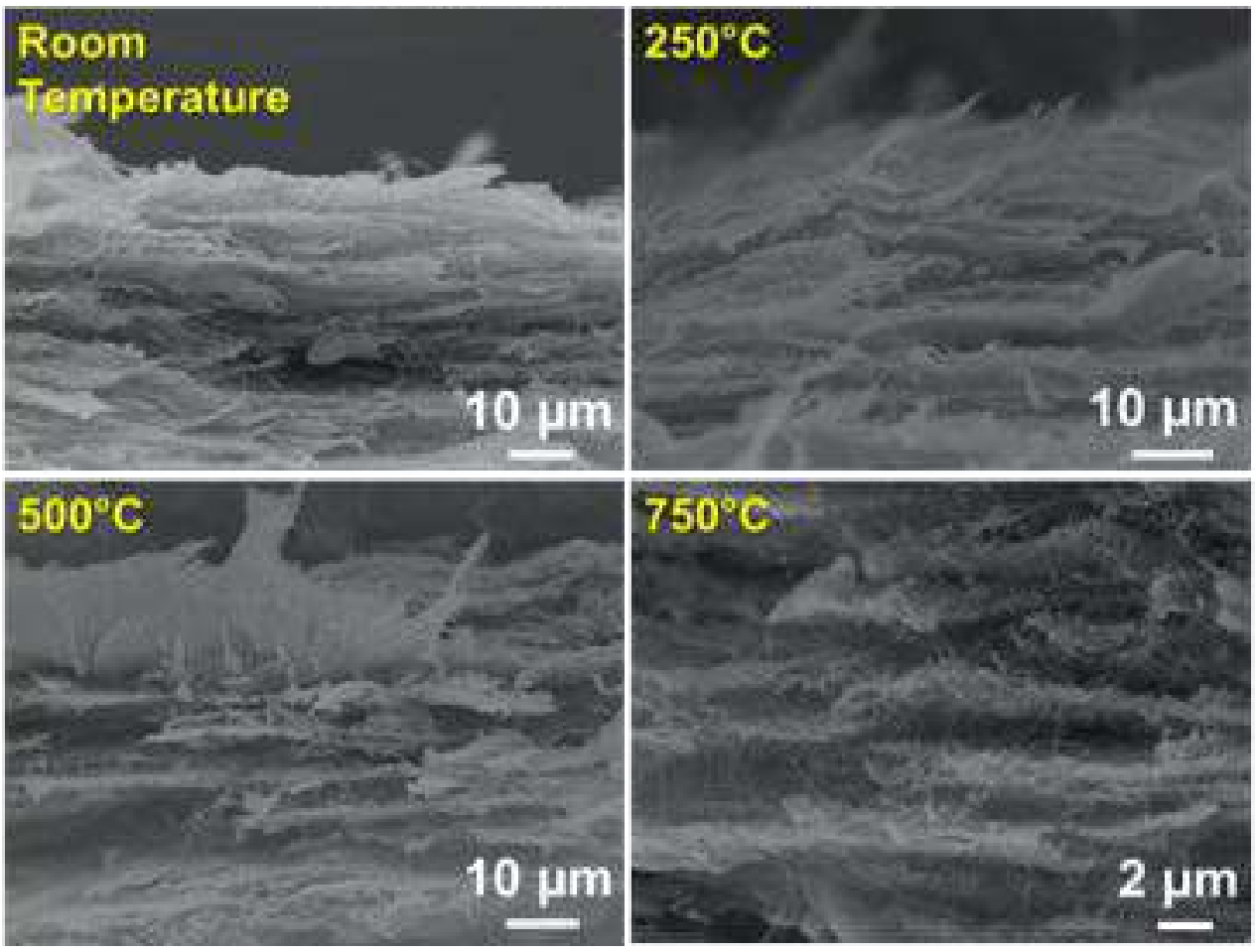

(b) High temperature morphological transformations
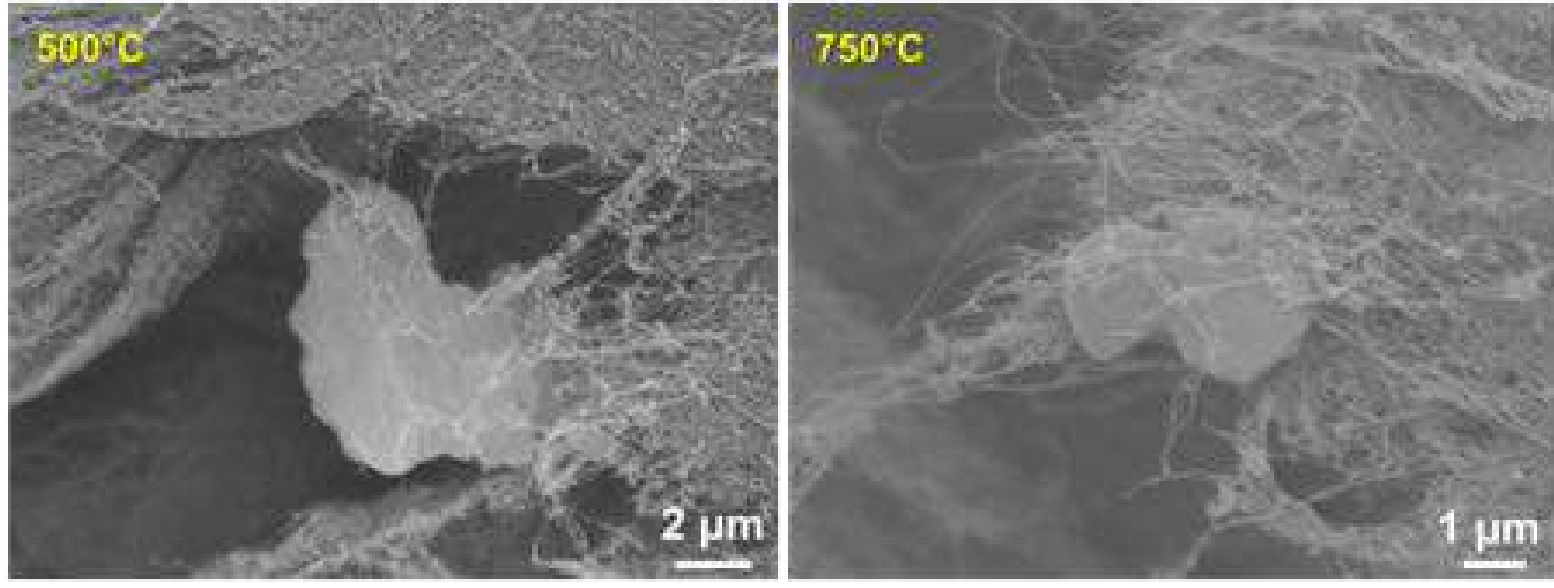
(c)

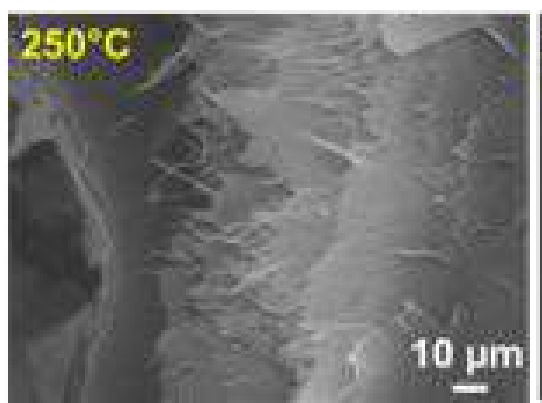

Realignment of BNNT bundles due to tension
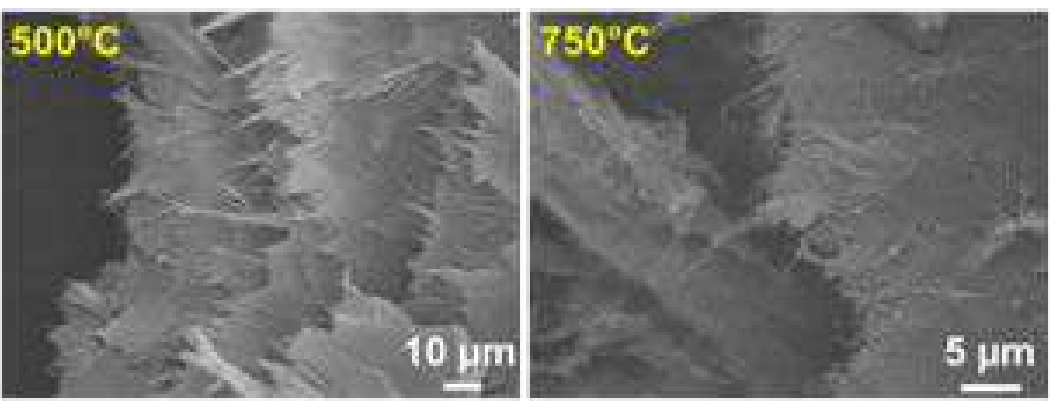

(d)

Transformation of inter-layer support mechanism
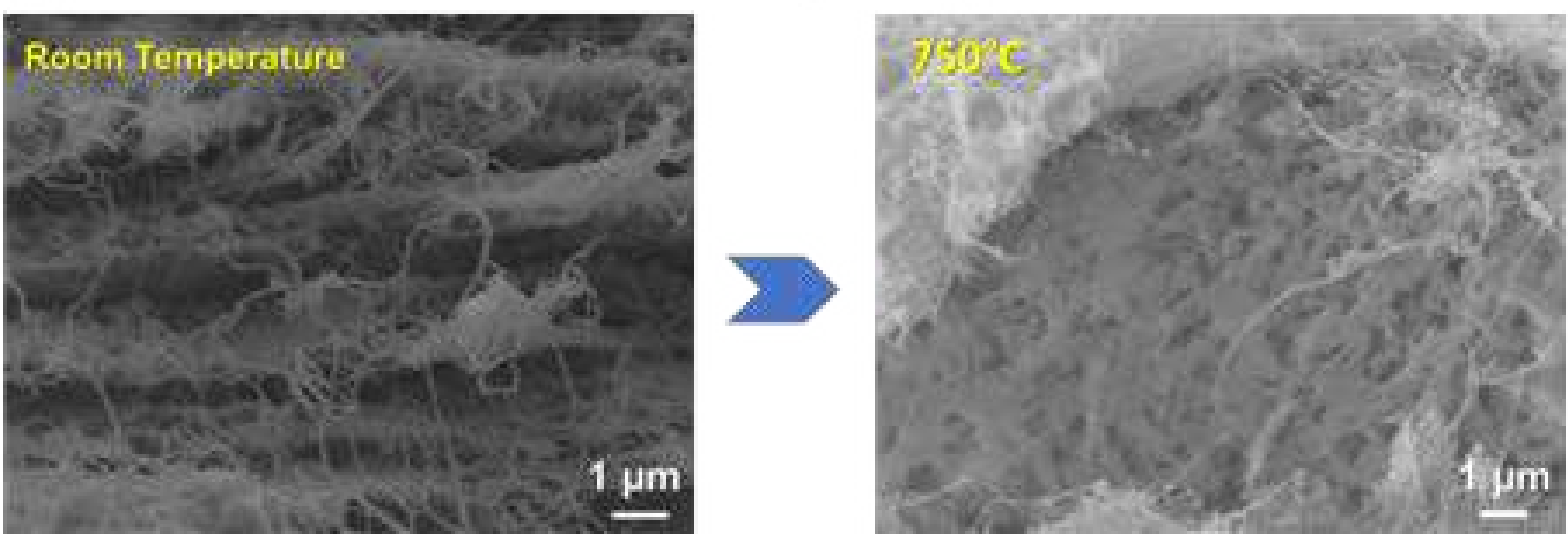

Figure 5.11. Scanning electron micrographs, revealing tensile failure mechanisms at different temperatures: (a) delamination of layers in the assembly, (b) morphological transformations at elevated temperatures, (c) alignment of nanotube bundles due to tensile loading, and (d) comparison of inter-layer load-bearing mechanism at room temperature and $750^{\circ} \mathrm{C}$.

During the tensile loading of BNNT, Stone-Wales (SW) defects are created after a critical mechanical strain ${ }^{27}$. SW defects sites are characterized by B-B and N-N homonuclear bonds ${ }^{10}$. These defect sites are susceptible to structural transformations due to bond rotation, creating dislocations in the nanotube. Therefore, the creation of defects during tensile deformation marks the onset of plastic yielding. The energy associated with SW defect $\left(E_{S W}\right)$ is related to tensile strain $(\varepsilon)^{27}$ : 
(a) Starting of the Test
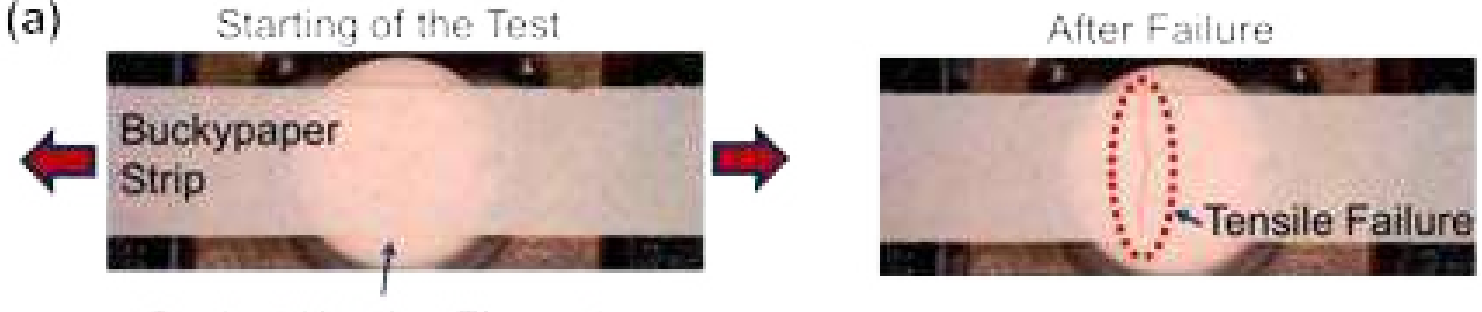

Contact Heating Element

(b) Thermal Imaging

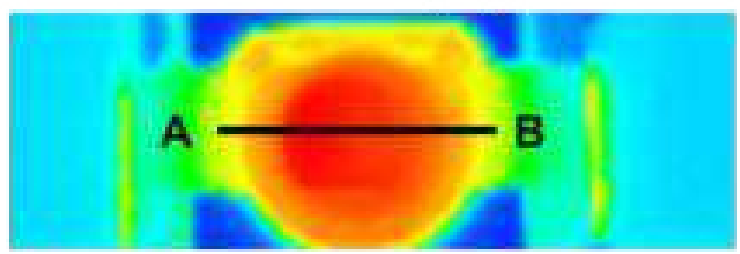

22.8

46.2

61.1

$218.8 \quad 539.7$

$\mathrm{T}\left({ }^{\circ} \mathrm{C}\right)$

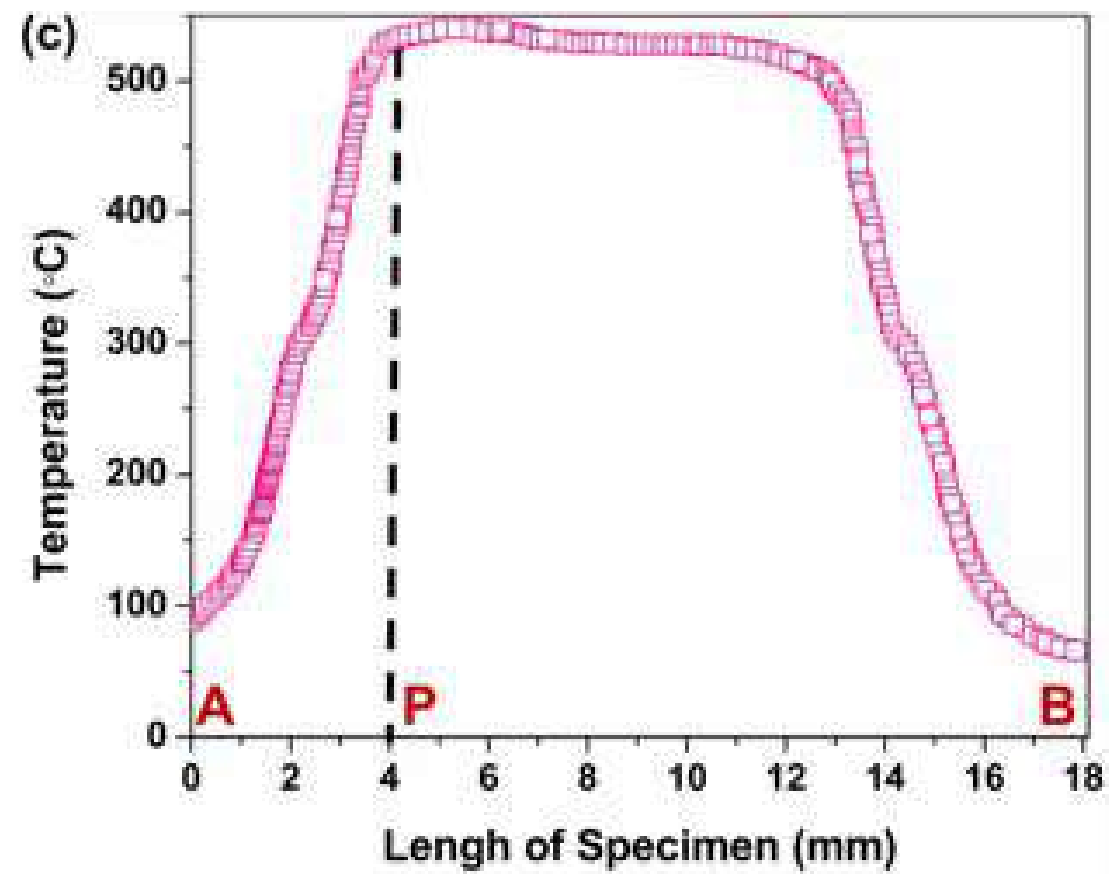

Figure 5.12. (a) Thermo-mechanical deformation of BNNT buckypaper exposed to a contact heating element during high temperature tensile testing, (b) infrared thermal image/ map of the buckypaper experiencing tensile loading, and (c) the corresponding temperature profile along the buckypaper length. 


$$
E_{S W}=5.6-10.8 \varepsilon-37.5 \varepsilon \sin \left(2 \chi+37.3^{\circ}\right)
$$

where $\chi$ is the chirality $\left(\chi \in\left[0^{\circ}, 180^{\circ}\right]\right)$. Based on Eq. 5.7, the formation of SW defects becomes energetically favorable at higher strains. Due to the nature of nanotube assembly in buckypaper, inter-tube slipping is expected to be prominent during tensile loading and the strains experienced by individual nanotube might not be high enough for defect formation. However, at elevated temperatures, the activation barrier for the SW defect goes down and therefore, the critical strain for plastic yielding also goes down. This explains the observed drop in failure strain for BNNT buckypaper as the deformation temperature increases (Figure 5.9). Mechanically induced defects can be created at lower tensile strains, thereby accentuating the plastic deformation.

At elevated temperatures, the buckypaper experiences complex thermo-mechanical conditions: tensile stresses induce re-alignment of the BNNT clusters, elevated temperatures create Stone-Wales defect sites in the nanotubes and selective morphological transformations alter the microstructure. In order to examine the influence of high temperature exposure in real-time, the tensile buckypaper specimen was heated to $500^{\circ} \mathrm{C}$ and the temperature gradient was probed by thermal imaging. In Figure 5.12a, the tensile set-up with a contact heater is shown. The buckypaper rests on the heater during the tensile test, such that the heater makes contact with the central region of the buckypaper strip. Simultaneous thermal imaging was performed during the tensile test at $500^{\circ} \mathrm{C}$. An instantaneous temperature map during the test is shown in Figure 5.12b. It is seen that the temperature of the specimen is high over the heating element $\left(\sim 540^{\circ} \mathrm{C}\right)$, but it drops near the grips or away from the heating element (points A and B marked in the image). The 
corresponding temperature profile is plotted in Figure 5.12c. It is noteworthy that the buckypaper is porous (very high porosity of $80 \%$ ), therefore the actual area/ volume that transfers the heat is limited. Additionally, heat loss/ dissipation to the atmosphere is also responsible for the drop in the specimen temperature in the regions not in direct contact with the heater. As a result, simultaneous thermal transport will take place during the tensile test, with heat flow from the hot central region to the relatively low-temperature outer regions of the buckypaper strip. The heat energy transfer is computed by Fourier's law:

$$
\dot{q}=-\lambda A(\Delta T / \Delta x)
$$

where $\dot{q}$ is the heat energy transfer per unit time, $\lambda$ is the thermal conductivity, $A$ is the cross-section area $(5 \mathrm{~mm} \times 80 \mu \mathrm{m}), \Delta T$ is the temperature difference across 2 points and $\Delta x$ is the distance between the two points. Considering the points $\mathrm{A}$ (near the grip) and $\mathrm{P}$ (over the heating element), the temperature gradient $(\Delta \mathrm{T})$ is $\sim 430^{\circ} \mathrm{C}$ and the heat transfer distance $(\Delta x)$ is $4 \mathrm{~mm}$. The thermal conductivity of porous materials $(\lambda)$ is determined by Loeb's relation ${ }^{28}$ :

$$
\lambda=\lambda_{s}(1-p)
$$

where $\lambda_{s}$ is the thermal conductivity of solid material and $p$ is the porosity. The reported peak thermal conductivity of 'aligned' BNNTs is $2400 \mathrm{~W} / \mathrm{m} \cdot \mathrm{K}^{29}$. As observed in Figure 5.12c, the nanotubes tend to align along the tensile axis during the deformation; therefore, this value of conductivity for aligned BNNT can be substituted to Eq. (5.9) for $\lambda_{\mathrm{s}}$. The inplane conductivity of the porous buckypaper is then computed to be $480 \mathrm{~W} / \mathrm{m}$.K from Eq. 
(5.9). Therefore, the heat flow rate is obtained to be $20.4 \mathrm{~W}$ (based on Eq. 5.8). The outof-plane thermal conductivity of the buckypaper was also determined by thermal flash diffusivity measurement and was found to be only $0.17 \mathrm{~W} / \mathrm{m} . \mathrm{K}$. This is anticipated because BNNT primarily conducts heat along its axial direction. During tensile deformation, the nanotubes align in the plane of the buckypaper, along the loading axis. Very few nanotubes are oriented perpendicular to the tensile loading plane, resulting in 3 orders of magnitude lower conductivity. The difference in thermal transport in and out of the buckypaper plane has implications for mechanical deformation behavior. As noted before, the plastic yielding of buckypaper is induced by Stone-Wales defects. Superior transport along the buckypaper will result in accentuated thermal migration of defects to form dislocations, activating plastic deformation mechanisms. Contrary to this, limited out-of-plane thermal transport results in arrested plasticity. This was evident from the SEM micrographs: Primarily nonplastic mechanisms such as delamination or detachment of nanotube pillars were prominent

for out-of-plane deformation (Figure 5.11a), as opposed to plastic mechanisms such as nanotube re-orientation, stretching and pull-out observed in the buckypaper plane (Figure 5.12c). Therefore, there is an interplay of multi-scale thermo-mechanical mechanisms during the tensile deformation of BNNT buckypaper, which manifests as multi-stage yielding and pop-ins in the stress-strain curves (Figure 5.9).

\subsubsection{Viscoelasticity at Elevated Temperatures}

The unique microstructure of buckypaper, with a network of nanotube junctions and struts, induces time-dependent mechanical characteristics. The buckypaper was subjected to 
dynamic tensile loading, with the mean axial displacement of $20 \mu \mathrm{m}$ and a displacement amplitude of $\pm 10 \mu \mathrm{m}$. The buckypaper was subjected to low deformations to avoid plastic yielding or failure of the assembly. Dynamic deformation results in a phase lag $(\delta)$ between the applied strain and the resultant stresses developed in the material ${ }^{30}$. This phase lag results in a complex deformation state: a component of the applied energy is stored as potential energy (elastic component), whereas some of the energy is dissipated during the loading cycle (loss component) ${ }^{31}$. The materials with significant loss component are viscoelastic in nature; the storage (E') and loss (E') moduli of such materials are expressed in terms of the phase lag angle, $\delta^{32}$ :

$$
\begin{aligned}
& E^{\prime}=\frac{\sigma}{\varepsilon} \cos \delta \\
& E^{\prime \prime}=\frac{\sigma}{\varepsilon} \sin \delta
\end{aligned}
$$

The ratio of loss modulus and storage modulus $\left(E^{\prime \prime} / E^{\prime}\right), \tan \delta$, is called the loss tangent and is a measure of the energy dissipation ability ${ }^{33}$. The dynamic mechanical analysis (DMA) of the buckypaper was performed from room temperature up to $750^{\circ} \mathrm{C}$. The loading/ unloading frequency-dependent loss behavior of the buckypaper is shown in Figure 5.13a for different temperatures. The buckypaper is characterized by excellent energy dissipation ability, with $\tan \delta$ value as high as $\sim 0.5$ at room temperature. The loss tangent drops as the temperature increases. Nevertheless, the material retains the viscoelastic nature even up to $750^{\circ} \mathrm{C}$ (with $\tan \delta$ recorded in the range of $\sim 0.05-0.1$ ).

During the dynamic loading-unloading cycle, the nanotube network experiences microstructure transformation by unzipping/ zipping of intertube junctions or nodes ${ }^{34}$. 
During the loading cycle, the nanotubes at the node separate (unzipping), resulting in a reduction in nanotube entanglements ${ }^{26}$. The unzipping process requires energy to overcome van der Waals attraction forces between BNNTs, resulting in energy dissipation. During the unloading cycle, the separated nanotubes come together at the nodal points (zipping of nodes). The zipping event is associated with the re-formation of inter-tube entanglements. This dynamic loss mechanism is elucidated in Figure 5.13b. The buckypaper can be considered to be a dense cluster of nanotubes. The strength of a nanoparticle cluster (with spherical nanoparticles) is modeled as ${ }^{35}$ :

$$
\sigma_{\text {cluster }}=\frac{1-p}{p} \frac{F}{d^{2}}
$$

where $p$ is the porosity, $F$ is the interparticle binding force and $d$ is the particle size (diameter in case of spherical particles). Eq. (5.12) can be modified for 1D nanoparticle clusters by replacing the $d^{2}$ term in the denominator with the product of tube diameter and length, $d * l$. Tang and co-workers determined the van der Waals attraction/ binding force (F) between two overlapping BNNTs to be $\sim 96 \mathrm{nN}$ by in-situ TEM investigation ${ }^{36}$. This binding force value corresponds to the nanotube diameter $(d) \sim 27.5 \mathrm{~nm}$ and overlapping length $(l) \sim 87 \mathrm{~nm}^{36}$. Substituting these values to the equation for $\sigma_{c l u s t e r}$, the strength of BNNT clusters is found to be $\sim 10 \mathrm{MPa}$, which must be overcome for the unzipping of nanotube junctions. During dynamic tensile loading, the buckypaper is subjected to a cyclic displacement $(\Delta x)$ of $10 \mu \mathrm{m}$. Therefore, the work done per unit area in a single loading cycle is computed by:

$$
\frac{W}{A_{r}}=\sigma_{\text {cluster }} \times \Delta x
$$




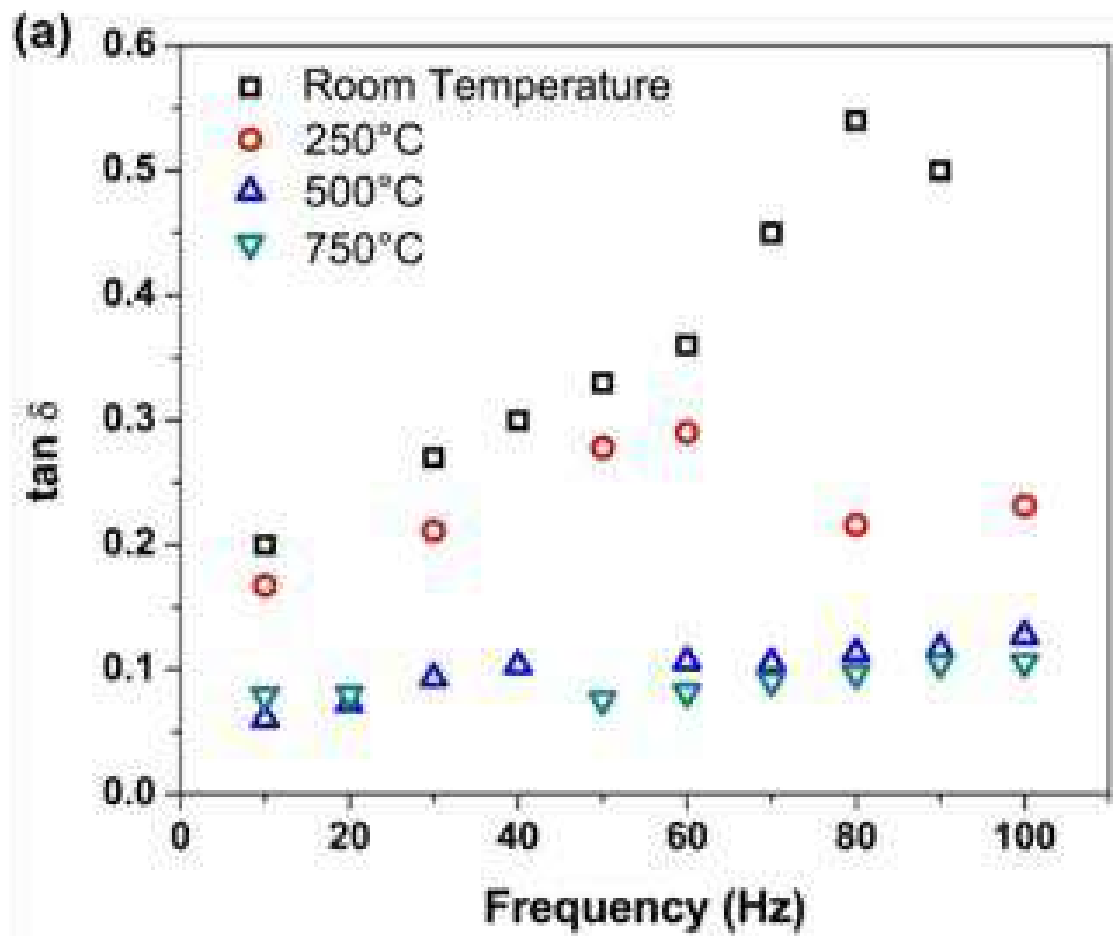

(b) MECHANISM FOR VISCOELASTICITY IN BNNT BUCKYPAPER

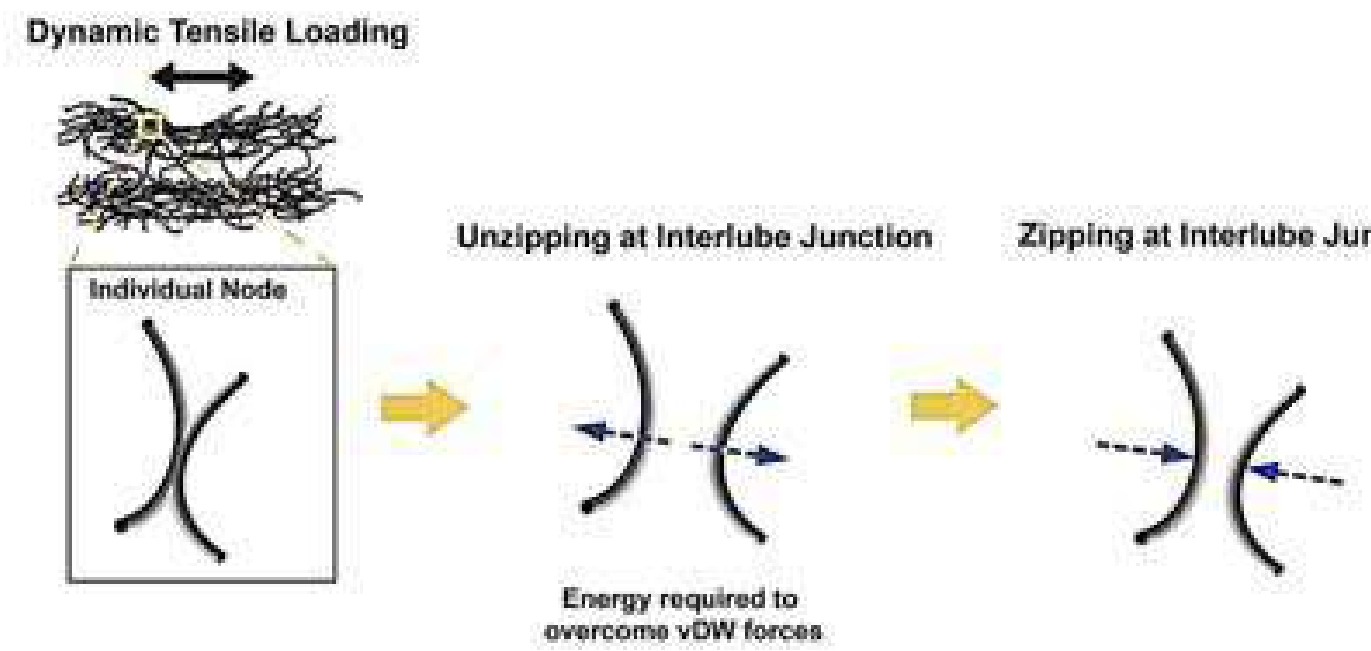

Figure 5.13. (a) Loss tangent as a function of dynamic loading frequency for BNNT buckypaper recorded at room temperature, $250^{\circ} \mathrm{C}, 500^{\circ} \mathrm{C}$ and $750^{\circ} \mathrm{C}$, and (b) the schematic illustration of the unzipping-zipping mechanism responsible for viscoelasticity in BNNT buckypaper assembly. 
This work of unzipping is computed to be $\sim 100 \mathrm{~J}$ per unit area, per loading cycle. This explains the remarkable energy dissipation ability observed in Figure 5.13a. At high frequency of dynamic mechanical tests, the loading-unloading becomes much faster. The increase in zipping-unzipping phenomena results in enhanced energy dissipation, which manifests as a generally rising trend for $\tan \delta$ with increasing frequencies (Figure 5.13a). A marginal drop in the value of loss tangent for frequencies above $80 \mathrm{~Hz}$ suggests the simultaneous activation of other deformation mechanisms, such as re-orientation/ alignment along loading direction, which start dominating over viscous mechanism (zipping-unzipping). During dynamic mechanical loading, frictional effects (atomic scale stick-slip instabilities) between sliding nanotubes can also play a role in energy dissipation. As the temperature increases, thermal excitations reduce stick-slip jumps ${ }^{22}$. Additionally, the mesh-like nanotube network is altered at elevated temperatures due to the coalescence of nanotubes shown in Figures 5.11b, d. As a result, there are fewer intertube junctions where zipping-unzipping phenomena take place during dynamic loading. This leads to suppressed loss tangent at temperatures exceeding $500^{\circ} \mathrm{C}$. Nevertheless, the retention of viscoelasticity even up to $750^{\circ} \mathrm{C}$ opens up windows for multifarious applications.

\subsection{Summary}

In summary, oxidative unzipping of BNNTs takes place at temperatures exceeding $750^{\circ} \mathrm{C}$. Multiple uncurled nanotubes in a dense cluster coalesce together to form stacks of 2D white graphene oxide platelets. Even for temperatures as high as $1000^{\circ} \mathrm{C}$, a significant proportion of nanotubes remain untransformed, confirming high oxidation resistance of BNNTs. Further, BNNTs display the ability to bear mechanical stresses up to $750^{\circ} \mathrm{C}$, with 
impressive damage tolerance upon multiple loading-unloading cycles. Superior thermal stability and mechanical performance of BNNT provide colossal opportunity to develop advanced nanocomposites, which is the subject matter of the subsequent chapters.

\section{Output: Published Articles}

The contents of this chapter are published as two journal articles, one on thermal transformations and the second on thermos-mechanical properties of BNNT:

(i) P. Nautiyal, A. Loganathan, R. Agrawal, B. Boesl, C. Wang, A. Agarwal, Oxidative unzipping and transformation of high aspect ratio boron nitride nanotubes into white graphene oxide platelets, Scientific Reports 6, 29498 (2016).

(ii) P. Nautiyal, C. Zhang, A. Loganathan, B. Boesl, A. Agarwal, High-Temperature Mechanics of Boron Nitride Nanotube "Buckypaper" for Engineering Advanced Structural Materials, ACS Applied Nanomaterials 2, $4402-4416$ (2019).

\section{References}

1. Chen, Y., Zou, J., Campbell, S. J. \& Caer, G. Le. Boron nitride nanotubes: Pronounced resistance to oxidation. Appl. Phys. Lett. 84, 2430-2432 (2004).

2. Zhang, Z., Guo, W. \& Dai, Y. Freestanding $(3,0)$ boron nitride nanotube: Expected to be stable well over room temperature. Appl. Phys. Lett. 93, 223108 (2008).

3. Zhi, C., Bando, Y., Tang, C. \& Golberg, D. Specific heat capacity and density of multi-walled boron nitride nanotubes by chemical vapor deposition. Solid State Commun. 151, 183-186 (2011).

4. Liao, Y. et al. Oxidative Etching of Hexagonal Boron Nitride Toward Nanosheets with Defined Edges and Holes. Sci. Rep. 5, 14510 (2015).

5. Shin, H. et al. Covalent Functionalization of Boron Nitride Nanotubes via Reduction Chemistry. ACS Nano 9, 12573-12582 (2015). 
6. Ferreira, T. H., Silva, P. R. O., Santos, R. G. \& Sousa, E. M. B. A Novel Synthesis Route to Produce Boron Nitride Nanotubes for Bioapplications. J. Biomater. Nanobiotechnol. 02, 426-434 (2011).

7. Tiano, A. L. et al. Boron nitride nanotube: synthesis and applications. NASA Techn, (2014).

8. Dreyer, D. R., Park, S., Bielawski, C. W. \& Ruoff, R. S. The chemistry of graphene oxide. Chem. Soc. Rev. 39, 228-240 (2010).

9. Sota, H. et al. Optical and electrical properties of boron nitride oxide films. Diam. Relat. Mater. 17, 826-829 (2008).

10. Miyamoto, Y., Rubio, A., Berber, S., Yoon, M. \& Tomànek, D. Spectroscopic characterization of Stone-Wales defects in nanotubes. Phys. Rev. B - Condens. Matter Mater. Phys. 69, 121413(R) (2004).

11. $\mathrm{Xu}, \mathrm{T}$. T. et al. Crystalline Boron Nanoribbons: Synthesis and Characterization. Nano Lett. 4, 963-9668 (2004).

12. Gulaczyk, I., Krȩglewski, M. \& Valentin, A. The N-N stretching band of hydrazine. J. Mol. Spectrosc. 220, 132-136 (2003).

13. An, W., Wu, X., Yang, J. L. \& Zeng, X. C. Adsorption and surface reactivity on single-walled boron nitride nanotubes containing stone-wales defects. J. Phys. Chem. C 111, 14105-14112 (2007).

14. Li, L. et al. High-quality boron nitride nanoribbons: Unzipping during nanotube synthesis. Angew. Chemie - Int. Ed. 52, 4212-4216 (2013).

15. Chen, B., Cheng, A. H. D. \& Chou, T. W. A Nonlinear compaction model for fibrous preforms. Compos. Part A Appl. Sci. Manuf. 32, 701-707 (2001).

16. Arif, M. F., Kumar, S. \& Shah, T. Tunable morphology and its influence on electrical, thermal and mechanical properties of carbon nanostructure-buckypaper. Mater. Des. 101, 236-244 (2016).

17. Golberg, D. et al. Direct force measurements and kinking under elastic deformation of individual multiwalled boron nitride nanotubes. Nano Lett. 7, 2146-2151 (2007).

18. Zobelli, A. et al. Defective structure of BN nanotubes: From single vacancies to dislocation lines. Nano Lett. 6, 1955-1960 (2006).

19. Schmidt, T. M., Baierle, R. J., Piquini, P. \& Fazzio, A. Theoretical study of native defects in BN nanotubes. Phys. Rev. B - Condens. Matter Mater. Phys. 67, 113407 (2003).

20. Nautiyal, P., Boesl, B. \& Agarwal, A. The mechanics of energy dissipation in a 
three-dimensional graphene foam with macroporous architecture. Carbon N. Y. 132, (2018).

21. Kim, K. S. et al. Polymer nanocomposites from free-standing, macroscopic boron nitride nanotube assemblies. RSC Adv. 5, 41186-41192 (2015).

22. Vanossi, A., Manini, N., Urbakh, M., Zapperi, S. \& Tosatti, E. Colloquium: Modeling friction: From nanoscale to mesoscale. Rev. Mod. Phys. 85, 529-552 (2013).

23. Nautiyal, P., Mujawar, M., Boesl, B. \& Agarwal, A. In-situ mechanics of 3D graphene foam based ultra-stiff and flexible metallic metamaterial. Carbon N. Y. 137, 502-510 (2018).

24. Embrey, L. et al. Three-Dimensional Graphene Foam Induces Multifunctionality in Epoxy Nanocomposites by Simultaneous Improvement in Mechanical, Thermal, and Electrical Properties. ACS Appl. Mater. Interfaces 9, 39717-39727 (2017).

25. Nautiyal, P., Zhang, C., Champagne, V. K., Boesl, B. \& Agarwal, A. In-situ mechanical investigation of the deformation of splat interfaces in cold-sprayed aluminum alloy. Mater. Sci. Eng. A 737, 297-309 (2018).

26. Li, Y. \& Kröger, M. Viscoelasticity of carbon nanotube buckypaper: Zippingunzipping mechanism and entanglement effects. Soft Matter 8, 7822-7830 (2012).

27. Dumitrică, T. \& Yakobson, B. I. Rate theory of yield in boron nitride nanotubes. Phys. Rev. B - Condens. Matter Mater. Phys. 72, 035418 (2005).

28. Smith, D. S. et al. Thermal conductivity of porous materials. J. Mater. Res. 28, 2260-2272 (2013).

29. Belkerk, B. E. et al. Thermal conductivity of vertically aligned boron nitride nanotubes. Appl. Phys. Express 9, 075002 (2016).

30. Lahiri, D., Das, S., Choi, W. \& Agarwal, A. Unfolding the damping behavior of multilayer graphene membrane in the low-frequency regime. ACS Nano 6, 39924000 (2012).

31. Nautiyal, P., Boesl, B. \& Agarwal, A. Harnessing Three Dimensional Anatomy of Graphene Foam to Induce Superior Damping in Hierarchical Polyimide Nanostructures. Small 13, 1-8 (2017).

32. Agrawal, R., Nieto, A., Chen, H., Mora, M. \& Agarwal, A. Nanoscale Damping Characteristics of Boron Nitride Nanotubes and Carbon Nanotubes Reinforced Polymer Composites. (2013). 
33. Nautiyal, P., Embrey, L., Boesl, B. \& Agarwal, A. Multi-scale mechanics and electrical transport in a free-standing 3D architecture of graphene and carbon nanotubes fabricated by pressure assisted welding. Carbon N. Y. 122, 298-306 (2017).

34. Xu, M., Futaba, D. N., Yamada, T., Yumara, M. \& Hata, K. Carbon Nanotubes with Temperature-Invariant Viscoelasticity from $-196^{\circ}$ to $1000^{\circ} \mathrm{C}$. Science (80-. ). 330, 1364-1368 (2010).

35. Rumpf, H. Agglomeration. (John Wiley, 1962).

36. Tang, D.-M. et al. Mechanical properties of bamboo-like boron nitride nanotubes by in situ TEM and MD simulations: Strengthening effect of interlocked joint interfaces. ACS Nano 5, 7362-7368 (2011). 


\section{CHAPTER 6 \\ PATHWAYS FOR ENGINEERING BORON NITRIDE NANOTUBE- ALUMINUM COMPOSITES}

This chapter presents a detailed study of BNNT-reinforced Al composites. Three different classes of processing techniques are used to engineer Al-BNNT composites: powder metallurgy (spark plasma sintering), solidification (casting), and additive manufacturing (plasma spray). These approaches are exploited to design nanocomposite microstructure with desirable features, such as aligned nanotubes, good interface wetting, covalent bonding between $\mathrm{BNNT}$ and $\mathrm{Al}$, and homogeneous dispersion of nanotubes in the matrix. BNNTs survived the high temperature and/or extreme pressure conditions involved in these processes. The effect of BNNT on mechanical properties is examined. The chosen manufacturing approaches provide the opportunity to unravel a vast processing space, such as state of metal (solid versus liquid), the timescale of interactions $\left(10^{-3}\right.$ to $\left.10^{+3} \mathrm{~s}\right)$, range of temperatures $\left(10^{2}\right.$ to $\left.10^{3 \circ} \mathrm{C}\right)$ and processing pressures $\left(10^{-1}\right.$ to $\left.10^{+1} \mathrm{MPa}\right)$. Therefore, this research advances the fundamental understanding of microstructure evolution and mechanical response necessary to engineer Al-BNNT composites with desired and predictable mechanical properties.

\subsection{Aluminum Reinforced with Aligned BNNTs by Sintering and Cold Working}

Ultra-long Boron Nitride Nanotubes (100-200 $\mu \mathrm{m})$ based layered Al-BNNT-Al composites were fabricated by spark plasma sintering, followed by cold rolling. The processing approach was discussed at length in Chapter 4. The BNNTs survived high 
temperature, pressure and severe deformation conditions of SPS and cold rolling, and also exhibited perfect alignment along the cold rolling direction. The subsequent sections detail the microstructure and mechanical behavior of the composite.

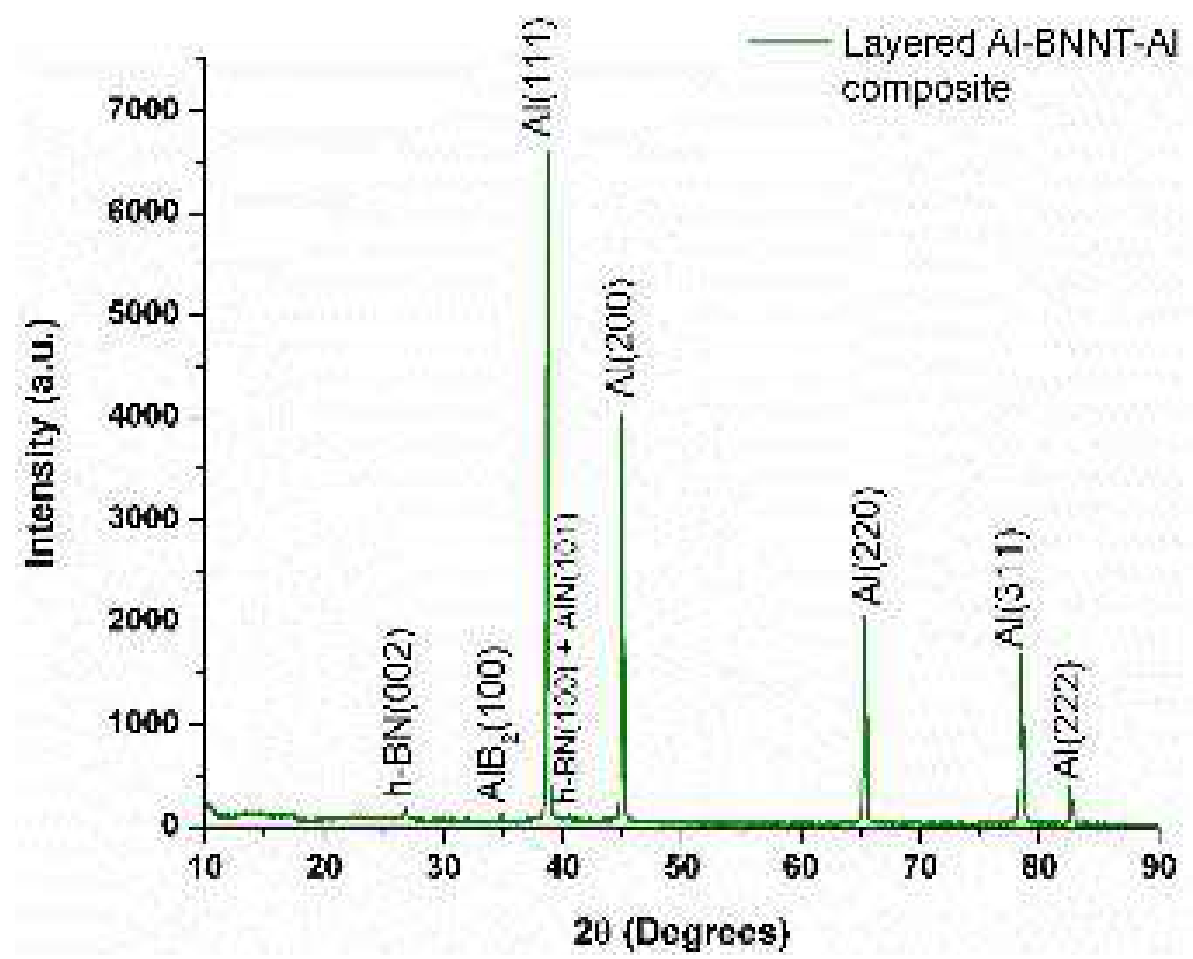

Figure 6.1. X-ray diffraction pattern for spark plasma sintered layered Al-BNNT-Al composite.

\subsubsection{Interfacial Reaction Products}

X-ray diffraction pattern of SPS sandwich composite is shown in Figure 6.1. Peaks corresponding to h-BN show that these long BNNTs survived high temperature $\left(575^{\circ} \mathrm{C}\right)$, high pressure (80 MPa) and long sintering time (1 hr) involved in SPS. In addition, weak peaks for $\mathrm{AlN}$ and $\mathrm{AlB}_{2}$ were detected, which suggests interfacial reactions taking place between $\mathrm{Al}$ and sandwiched BNNT layer. It is noteworthy that Lahiri et al. ${ }^{1}$ did not report any interfacial product formation for bamboo-shaped BNNT reinforced Al composite 
consolidated by SPS. This could be related to lower sintering temperatures $\left(500^{\circ} \mathrm{C}\right)$ employed in that study. Yamaguchi et al.'s study ${ }^{2}$ involved SPS at $550^{\circ} \mathrm{C}$; but since the sintering time was relatively low ( $20 \mathrm{~min})$, they too did not detect any reaction product. Lahiri and co-workers reported interfacial products for furnace sintering in Ar atmosphere, but the reaction was slow, and new products could be noticed only after 2 hours of soaking ${ }^{3}$. In the present work, the sputter-coating of the Al layer on the BNNT mat enhanced the possibility of interfacial reactions because of intimate metal/nanotube bonding. Moreover, the long BNNT mats have a large interfacial area of contact due to small nanotube diameter, and longer sintering time $(1 \mathrm{~h})$ promoted the formation of trace amount of $\mathrm{AlB}_{2}$ and $\mathrm{AlN}$.

\subsubsection{Tensile Properties}

The tensile stress-strain plot for the Al-BNNT sandwich composite is shown in Figure 6.2. BNNT reinforcement resulted in a highly impressive $400 \%$ increase in composite tensile strength to $200 \mathrm{MPa}$, as compared to the modest tensile strength of just $40 \mathrm{MPa}$ for pure $\mathrm{Al}^{4}$. The composite strength for Al-BNNT in this study is, in fact, higher than many commercial Al alloys, like Al1100 (90 MPa), Al3003 (110 MPa), Al6061 (125 MPa) and Al2024 (185 MPa) ${ }^{5}$. The sandwich hybrid showed a three-stage yielding (Figure 6.2). This layered composite consists of $\mathrm{Al}, \mathrm{BNNT}$ mat, and interfacial products ( $\mathrm{AlN}$ and $\left.\mathrm{AlB}_{2}\right)$. While BNNT has the highest tensile and yield strength, pure Al is least strong. It can be

assumed that $\mathrm{AlN}$ and $\mathrm{AlB}_{2}$ ceramic products formed at the interface would be having strength intermediate to $\mathrm{Al}$ and BNNT. Hence, the first yield strength can be ascribed to $\mathrm{Al}$, the second regime in the stress-strain curve is most likely related to 
debonding/separation of interfacial products from BNNT layer, and the third yield point is associated with sandwiched BNNTs. Although the tensile strength of individual nanotube has been reported to be $\sim 30 \mathrm{GPa}^{6}$, the same is not realized here since the sandwiched BNNT layer consists of multiple long BNNTs held together by van der Waals attractive forces. When such loosely knit fibers are pulled apart, they would break at the points of interlock at comparatively lower stresses. The multi-stage nature of yielding is discussed in detail in

\section{Section 6.1.4.}

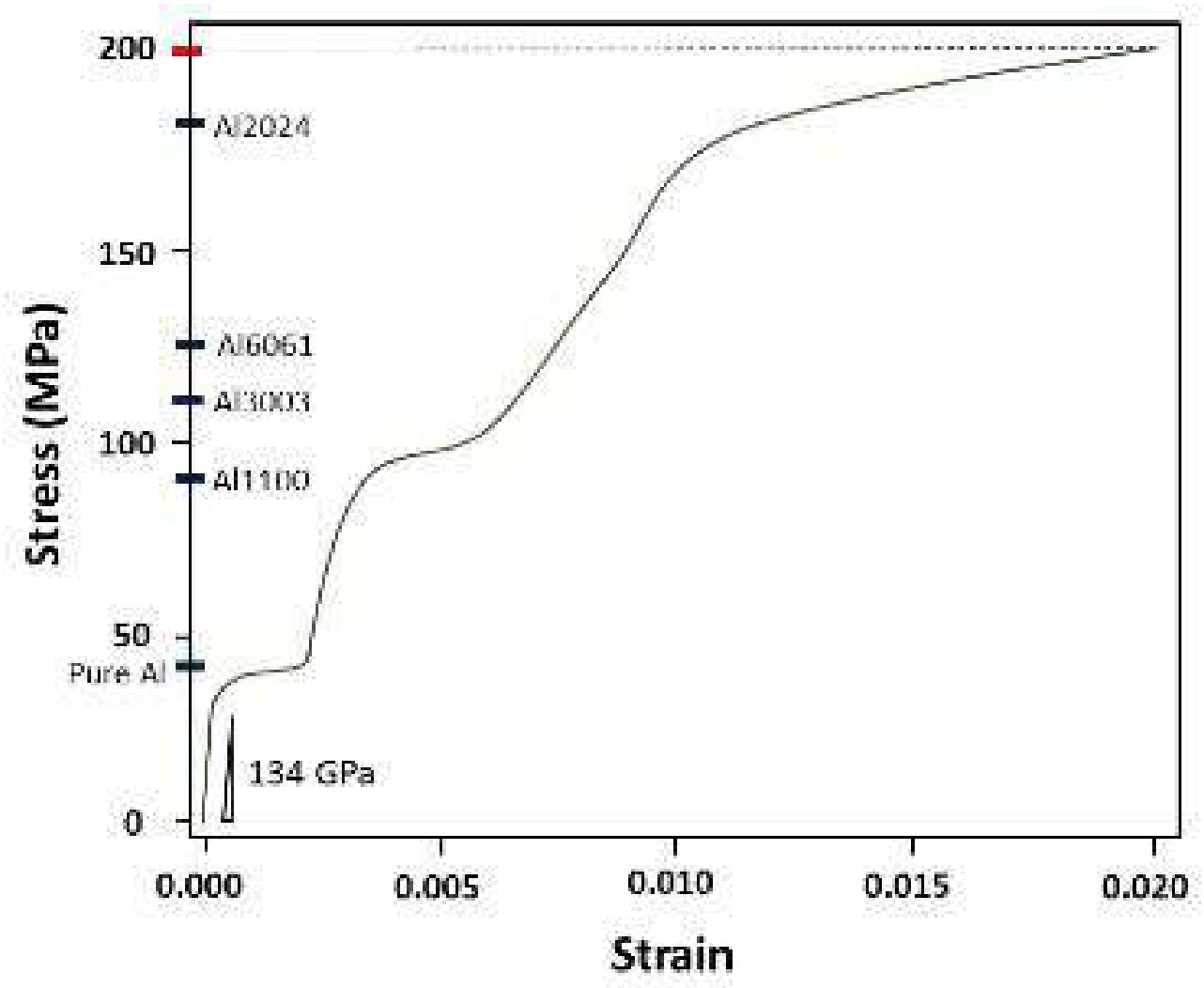

Figure 6.2. Tensile stress-strain behavior for layered Al-BNNT-Al composite showing multi-stage yielding and significant strengthening.

The elastic modulus of the hybrid composite was determined by computing the slope of the linear initial region of the stress-strain plot. Young's modulus was obtained to 
be $134 \mathrm{GPa}$ (Figure 6.2), which is almost double the elastic modulus of pure $\mathrm{Al}$ ( 70 $\mathrm{GPa})^{4}$. This shows that the BNNT layer not only improves composite's fracture strength, but also enhances the rigidity in Hooke's law regime. The failure elongation of these composites is low (2\% strain). This can be improved by more careful composite engineering, modification of cold rolling parameters and heat treatment of the sample. However, the most striking observation here is the significant strengthening and stiffening of these sandwich composites due to high aspect ratio BNNTs.

\subsubsection{Fracture Surface Examination}

SEM image of the fracture surface in Figure 6.3 shows nanotubes protruding from the interface between $\mathrm{Al}$ and BNNT mat. This image provides visual confirmation of the survival of BNNTs even after being subjected to high temperature and pressure conditions of SPS, as well as heavy plastic deformation induced during cold rolling. The zone enclosed by red color in the figure shows the interfacial region between the $\mathrm{Al}$ layer and BNNT mat. It can be seen that some of the nanotubes in the BNNT mat act as anchors (encircled by yellow color) to link strongly with the Al layer. This is a signature of strong bonding between the $\mathrm{Al}$ and BNNT layer, which is indispensable for enhanced strengthening of the layered composite. BNNTs are pulled out along the tensile loading direction, indicating that the long nanotubes fractured prior to the failure of the composite. The inset of Figure 6.3 shows the typical cup and cone ductile failure signature in Aluminum. While nanotubes fail by fracture mode, the Al matrix undergoes ductile deformation. 


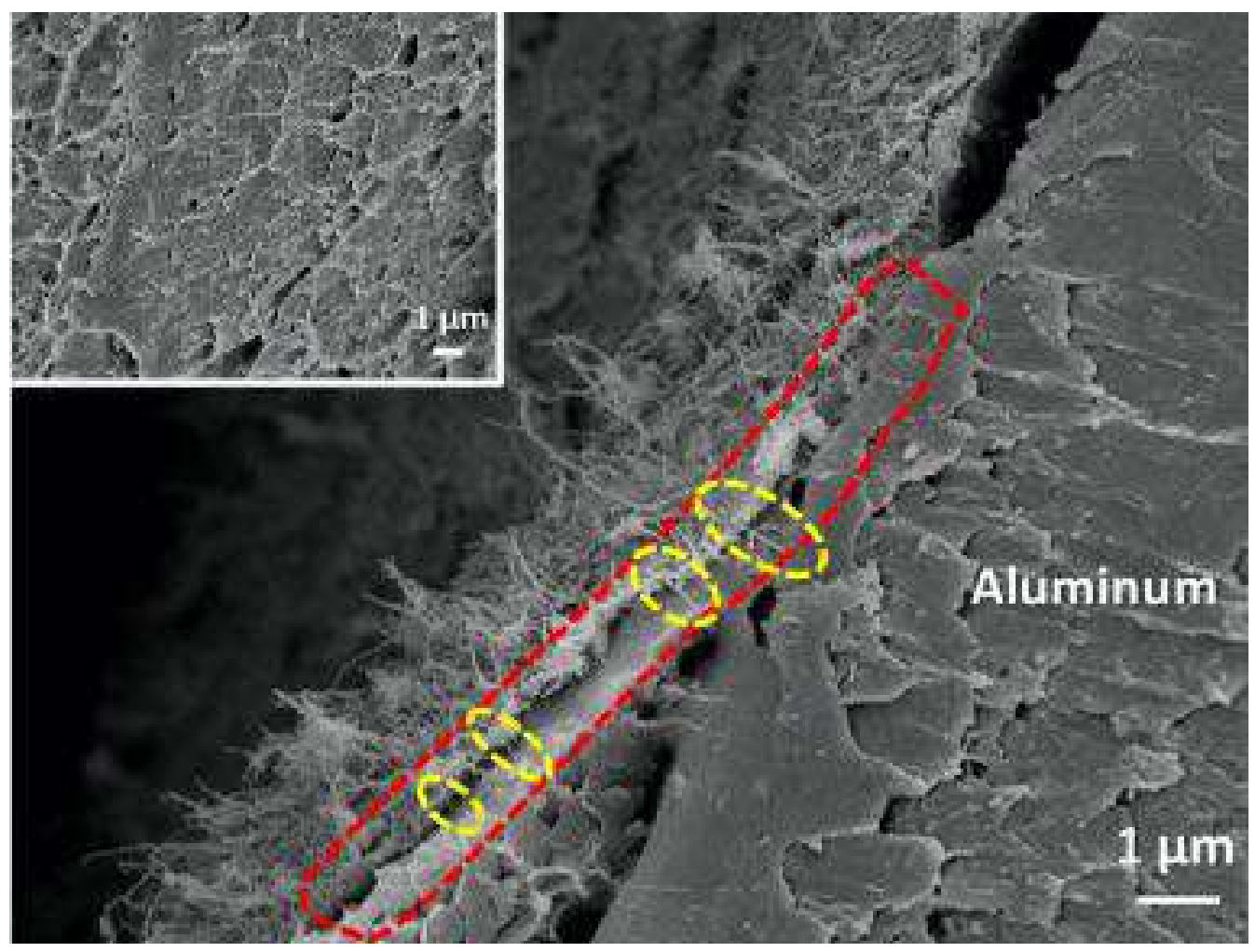

Figure 6.3. SEM image showing composite fracture surface, with BNNTs pulled out along the tensile axis. Inset shows the typical ductile mode of failure shown by Aluminum.

Strengthening in this sandwich composite is taking place due to load transfer from the matrix layer to the long nanotubes. Therefore, in the post-tensile sample, the Al layer was manually delaminated, to expose the Al/BNNT interface. SEM investigation of the exposed interface was then carried out to probe the deformation mechanism. Figure 6.4a shows the pulled out nanotubes acting as crack bridges. They bridge the Al-BNNT clusters ruptured due to tensile loading. These nanotubes are oriented along the tensile loading direction, which is also the cold rolling direction. The perfect alignment of BNNTs in the desired orientation is responsible for tremendous strengthening noticed in the stress-strain curve. Figure 6.4b shows the enlarged view of the Al-BNNT layer interface. A thin layer 

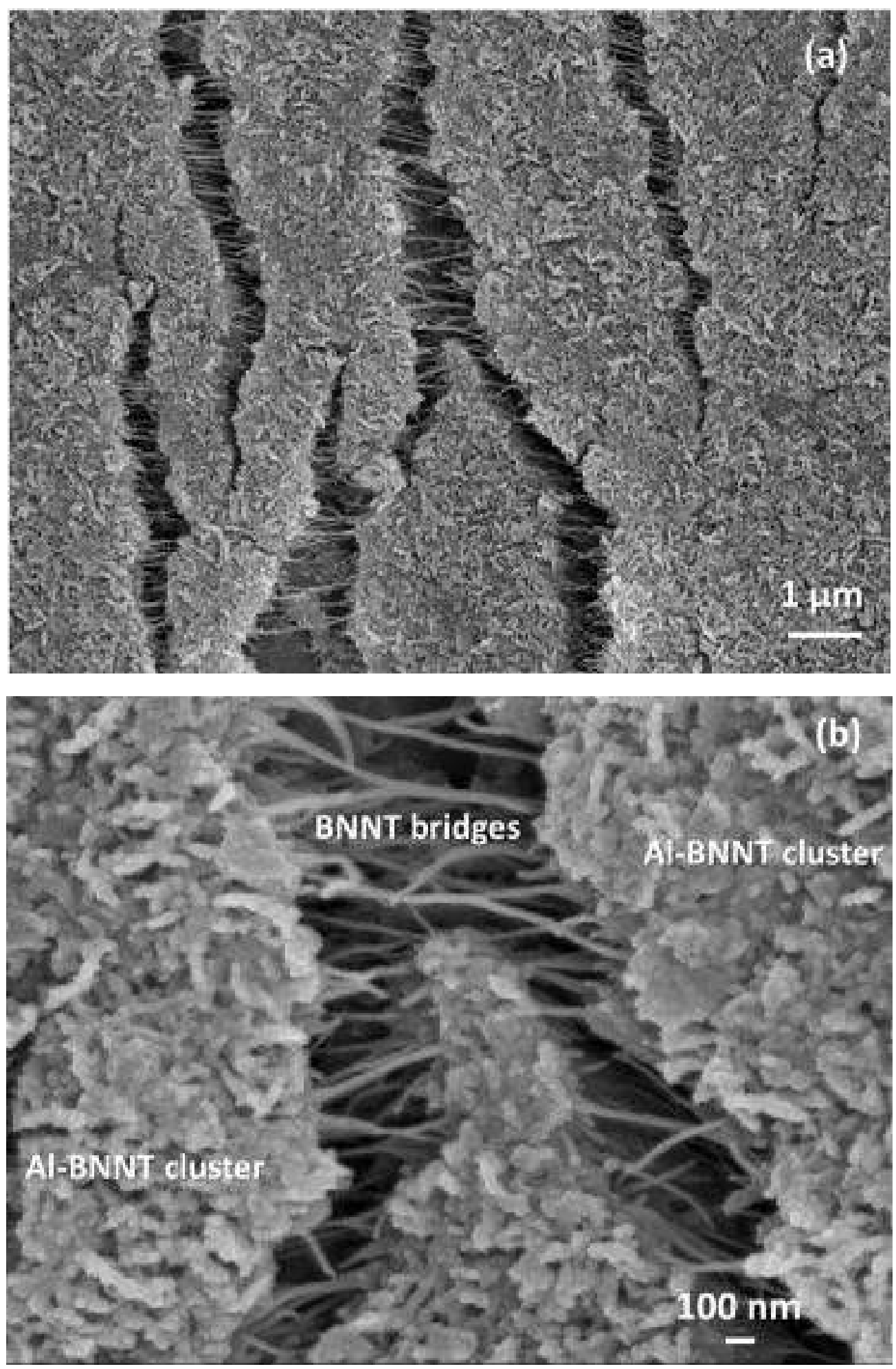

Figure 6.4. SEM images showing: (a) crack-bridging due to directionally aligned BNNTs, and (b) enlarged view of a BNNT network acting as crack bridges along the entire depth of the crack section. 
of sintered Aluminum adhered to the BNNT cluster can be seen. Such an excellent adherence of $\mathrm{Al}$ on $\mathrm{BNNT}$ is aided by the sputter coating of $\mathrm{BNNT}$ by $\mathrm{Al}$ prior to sandwiching. Even after the tensile rupture of the sandwich composite, the long fibers hold the structure together by highly effective bridging of cracks. As can be seen from the SEM image, the nanotube connections are extensively spread across the entire depth of crack cross-section.

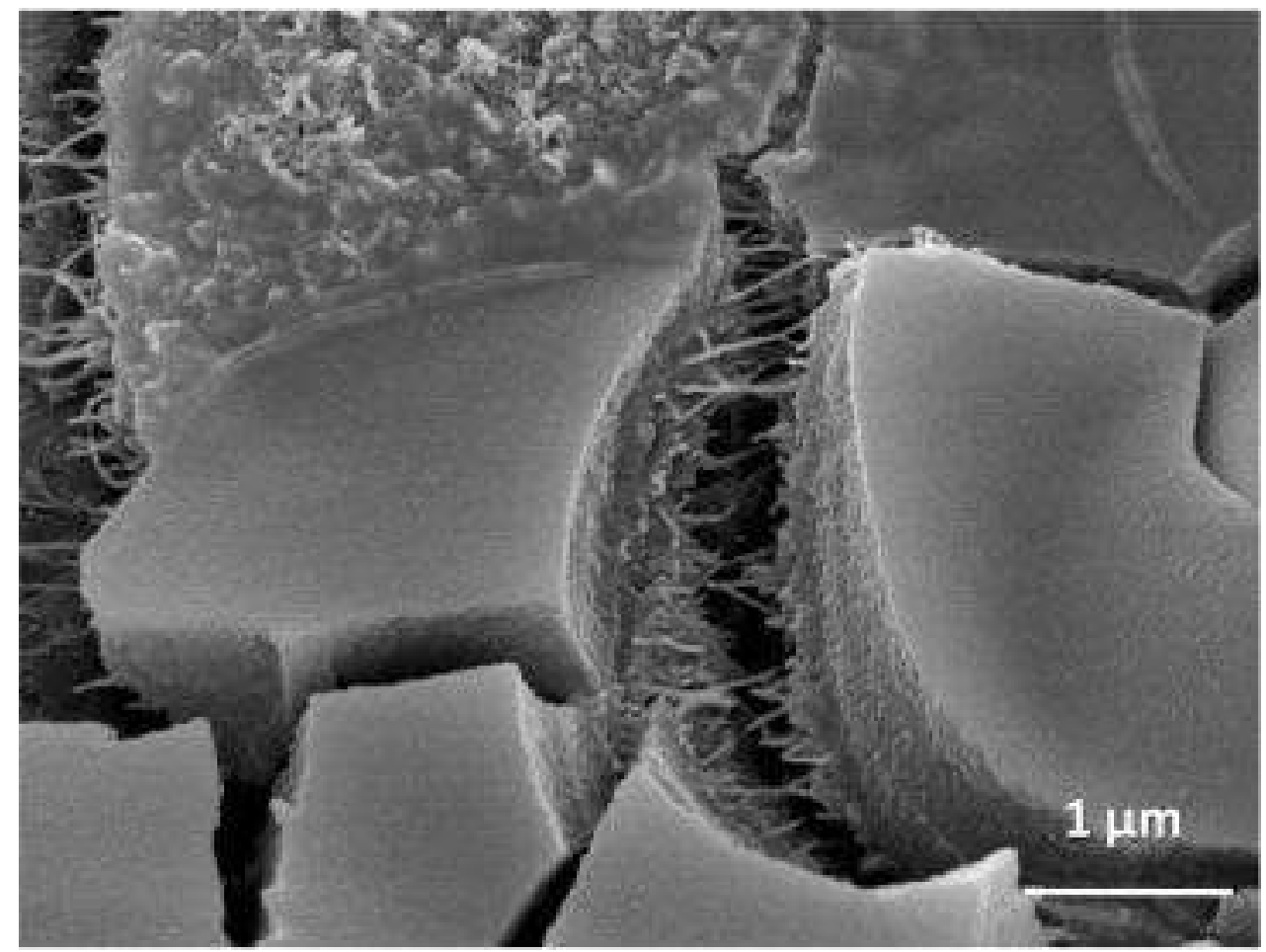

Figure 6.5. SEM image showing detachment of interfacial product and pulled out nanotubes along $\mathrm{Al} / \mathrm{BNNT}$ interface after tensile failure.

In some of the regions along the interface, reaction products were observed. Posttensile SEM image of the interfacial product is shown in Figure 6.5. The image shows the debonding of the interfacial product layer from the nanotube surface. Also, some of the pulled out nanotubes bridge these interfacial products. These features can be demarcated 
from pure Al by the nature of the failure. Characteristic ceramic style cracking is observed. The directionally aligned ultra-long BNNTs act as crack bridges, and lead to the toughening of the interfacial layer. These SEM images corroborate the findings from the stress-strain plot.

\subsubsection{Discussion of Strengthening Mechanisms}

In fiber-reinforced composites, the load is transferred from matrix to fibers through interfacial shear stress (shear lag model) ${ }^{7}$. Superior load transfer and better strengthening are expected when fibers are long due to higher interfacial area between fiber and matrix. It was mentioned in Chapters 1 and 2 that fiber reinforcement can be utilized to its full capability when fiber length exceeds a critical length, $1_{c}{ }^{7}$ :

$$
l_{c}=\frac{\sigma_{f} d}{\sigma_{m}}
$$

where $d$ is the fiber diameter, $\sigma_{f}$ is the fiber strength and $\sigma_{m}$ is the matrix strength. BNNT has a reported strength of $\sim 30 \mathrm{GPa}$. Pure Al has strength $\sim 40 \mathrm{MPa}^{4}$. BNNTs employed in this study have a reported diameter of 3-6 nm. For calculation purposes, we can assume $d$ to be the mean value, that is, $4.5 \mathrm{~nm}$. Based on Eq. (6.1), critical BNNT length is obtained to be $\sim 3.4 \mu \mathrm{m}$. BNNTs employed in this study are as long as $\sim 200 \mu \mathrm{m}$, which far exceeds the calculated value of $l_{c}$. It is due to this reason that ultra-long BNNT reinforced sandwich composite in this study exhibited significantly enhanced mechanical properties (Figure 6.2). For composites reinforced with long fibers $\left(l>l_{c}\right)$, strength is given by ${ }^{8}$ :

$$
\sigma_{c}=\sigma_{f} V_{f}\left(1-\frac{l_{c}}{2 l}\right)+\sigma_{m}^{f}\left(1-V_{f}\right)
$$


where $\sigma_{c}$ is the composite fracture strength, $\sigma_{m}^{f}$ is the strength of matrix at the failure strain of the composite, $V_{f}$ is fiber volume fraction in the composite and $l$ is the average fiber length. Based on Eq. (6.2), it can be argued that the greater the average BNNT length, the higher would be the expected strengthening of the composite. SEM images of interface attest to the presence of a network of aligned ultra-long nanotubes, which explains $400 \%$ enhancement of strength for the composite hybrid. The exact theoretical value of strength using Eq. (6.2) is not computed here because of two reasons: First, non-availability of statistical data for averaging BNNT length $(l)$, and secondly, the shear lag model does not take into consideration interfacial product formation and nanotube clustering. Therefore, strength value determined using the above equation is not characteristic strength for the hybrid composite studied here. However, this model gives a fair estimate of the influence of nanotube length on composite strength. This explains why the high aspect ratio BNNTs used here are favorable reinforcement.

The strength of metal matrix composites strongly depends on physical-mechanical interactions as well as chemical reaction(s) taking place between the metal and the nanotubes. Sputtering of $\mathrm{Al}$ on the BNNT mat leads to the formation of the Al-BNNT cluster, as shown in Figure 6.4b. These metal-nanotube clusters assist in load bearing ${ }^{7,9}$. The sandwiched BNNT mat with adhered Al can be considered as a localized microcomposite by itself (but with a higher fraction of nanotubes). XRD result (Figure 6.1) and SEM image (Figure 6.5) also attest to the formation of $\mathrm{AlN}$ and $\mathrm{AlB}_{2}$ due to reaction at the interface. Interfacial products can improve the bonding between the metal matrix and nanotubes. For instance, $\mathrm{Al}$ and $\mathrm{AlN}$ have stable orientation relationship ${ }^{3}$, which 
strengthens the matrix-BNNT bond. This leads to superior load transfer capability. This manifests as multi-stage yielding in the stress-strain plot, where first yield point corresponds to $\mathrm{Al}$ yielding (inset of Figure 6.3), the second regime is associated with failure of Al-BNNT clusters (Figure 6.4) and interphases (Figure 6.5), and the third regime is due to nanotube failure (pull out in Figure 6.3). The shear lag model proposed by Ryu et al. ${ }^{10}$ provides mathematical relations for matrix as well as nanotube cluster yield points. Matrix yield stress is given by:

$$
\sigma_{y, m}=\frac{V_{f} \sigma_{m}}{2} S_{e f f}+\sigma_{m}
$$

where $S_{\text {eff }}$ is the effective aspect ratio of an elongated nanotube cluster oriented at an angle $\theta$ to the loading direction. The average value of $S_{\text {eff }}$ is obtained as:

$$
S_{e f f, A v}=\int_{0}^{\pi / 2} S_{e f f}(\theta) F(\theta)(2 \pi \sin \theta) d \theta
$$

where $F(\theta)$ is the probability distribution function of the misorientation of the BNNT/Al clusters. It is noteworthy that BNNTs in the fabricated hybrid are all aligned along the cold rolling direction, which was attested by SEM images of the interface (Figure 6.4). The tensile loading direction was also similar to the rolling direction. Therefore, the value of $\theta$ can be approximated to zero. As a result, the $S_{\text {eff }}$ term would be zero too (based on Eq. 6.4). This implies the yield point is the same as the matrix strength (Eq. 6.3). This is corroborated by the stress-strain plot, where the first yield point is close to $40 \mathrm{MPa}$, which is also the strength of pure Al. This, in turn, verifies our claim that the novel hybrid structure fabricated in this study has perfectly aligned nanotubes, oriented along the rolling direction, since experimental as well as theoretical values perfectly match for $\theta=0$. 


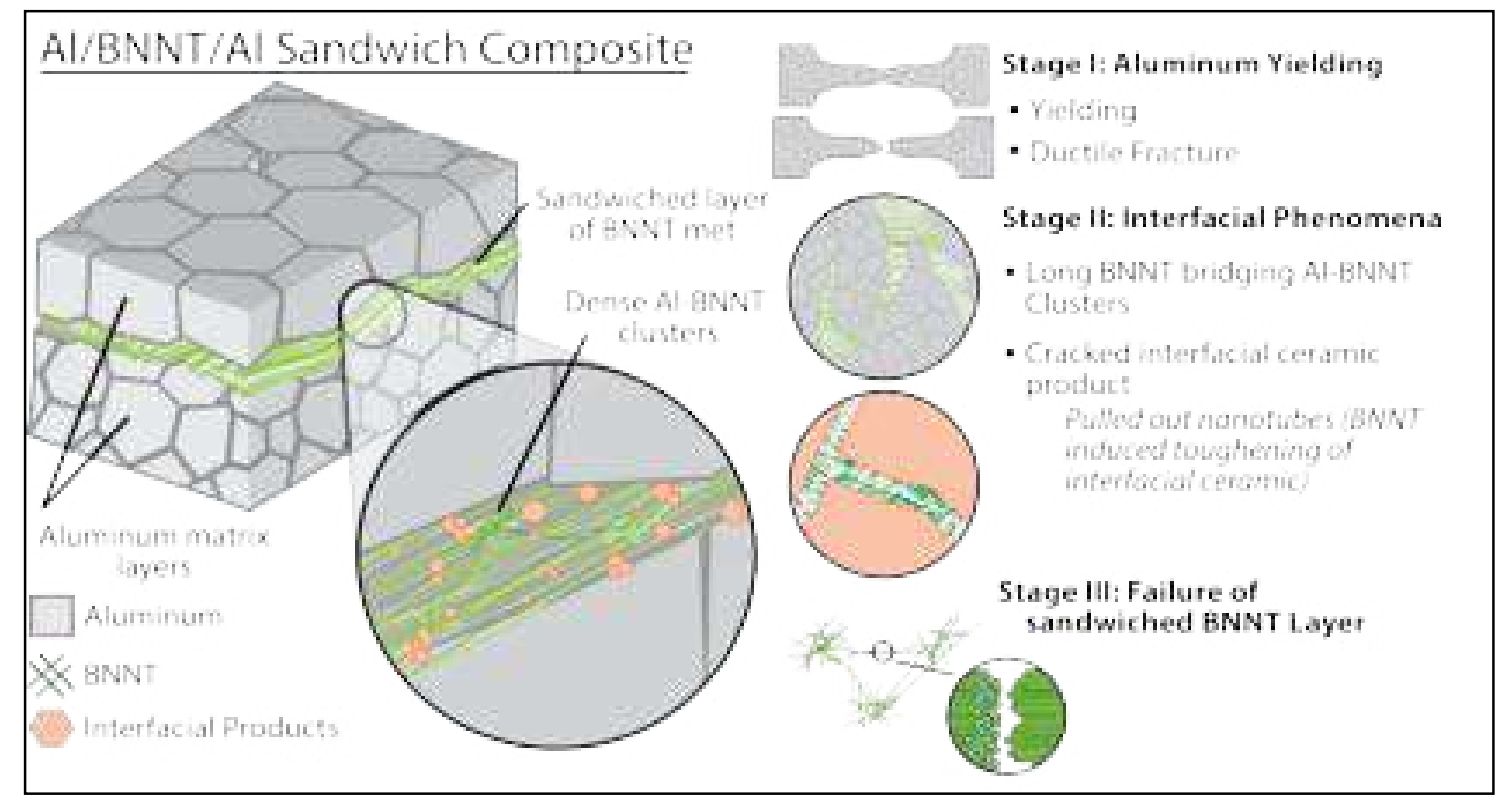

Figure 6.6. Schematic diagram summarizing strengthening and deformation mechanisms in the Al-BNNT-Al sandwich composite.

The secondary yield point associated with the nanotube cluster is typically modeled by the simple rule of mixture formula ${ }^{7}$. However, the model does not take into consideration strengthening due to interphase(s). Since the interfacial deformation in this sandwich composite is due to a combination of Al-BNNT cluster and interfacial product failure, Ryu et al.'s model ${ }^{10}$ cannot be applied directly for secondary yield point calculations. However, from the experimental stress-strain plot shown in Figure 6.2, secondary yielding associated with interfacial phenomenon takes place at $~ 100 \mathrm{MPa}$ which is attributed to combined phenomena of failure of the Al-BNNT cluster and reaction product failure and debonding.

Tertiary yielding occurs due to the stretching of nanotubes, which leads to BNNT bridge formation as shown in Figure 6.4. These stretched BNNTs bridge the cracked Al- 
BNNT clusters. Yielding of directionally aligned ultra-long BNNT mats was found to initiate at $\sim 167 \mathrm{MPa}$ (Figure 6.2). The final rupture of the sandwiched composite took place at $200 \mathrm{MPa}$, due to fracture failure of the nanotubes (Figure 6.3).

Strengthening and deformation mechanisms in this composite are summarized schematically in Figure 6.6.

\subsection{Reactive Interface Engineering in Al-BNNT by Equilibrium Solidification}

BNNTs are mixed in molten $\mathrm{Al}$ to prepare a metal matrix composite by equilibrium solidification route. Interfacial phenomena and reactions between nanotubes and molten $\mathrm{Al}$ are investigated. The microstructure evolution due to equilibrium solidification is correlated with the mechanical response captured from the nanocomposite microstructure.

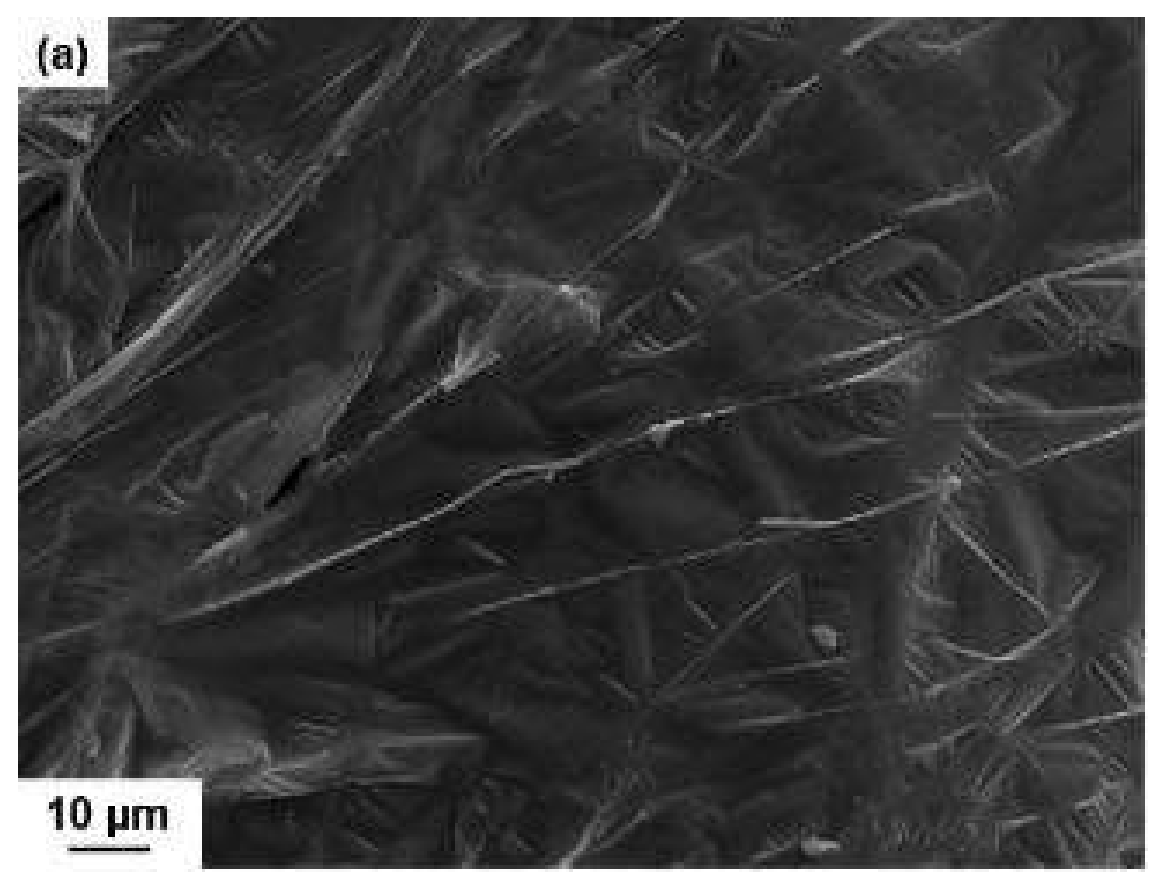



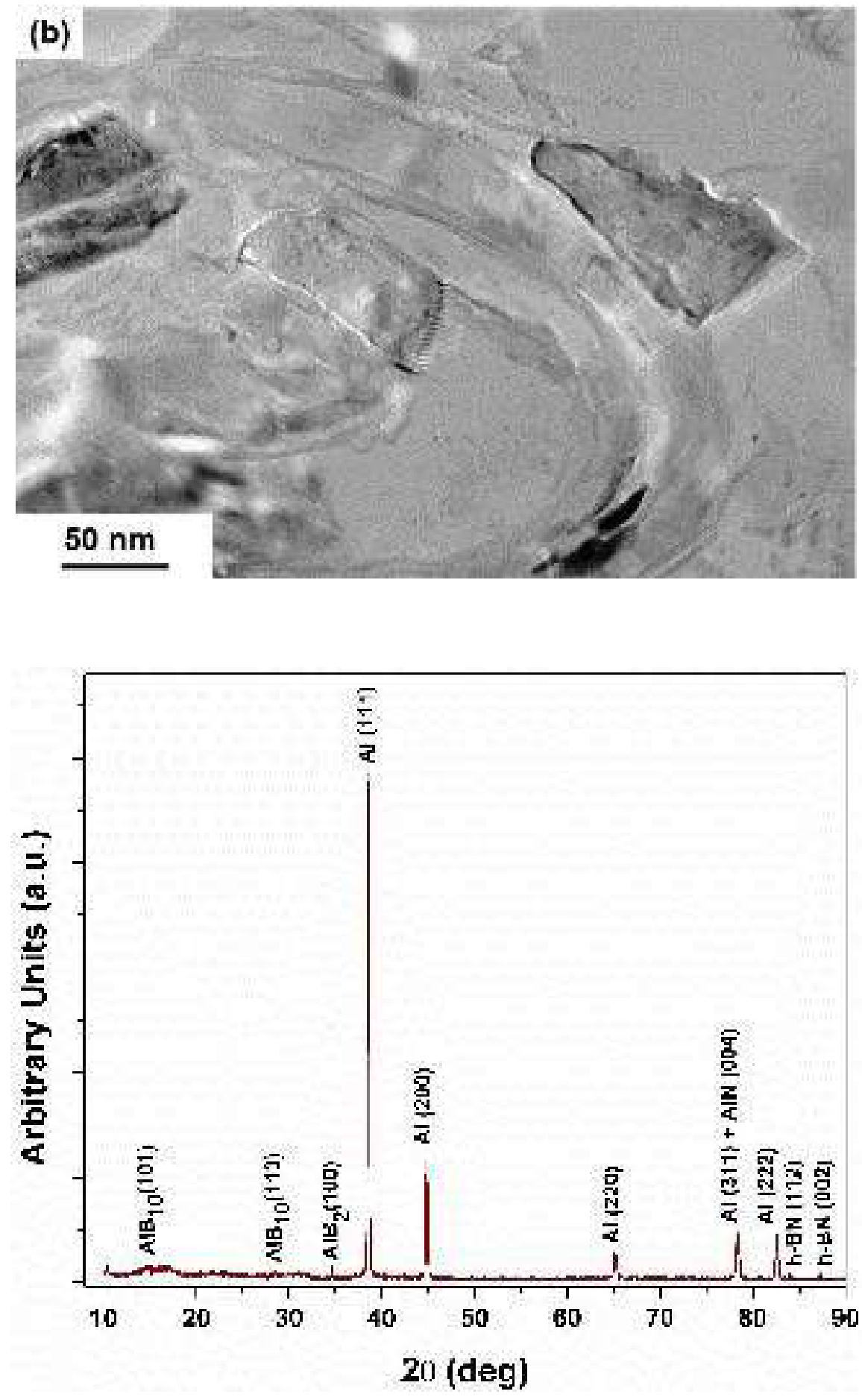

Figure 6.7. (a) SEM image showing a network of long nanotube clusters well meshed in Al matrix post solidification, (b) low magnification TEM image showing long and intact BNNTs in cast Al-BNNT composite, and (c) phases identified in solidified Al-BNNT composite. 


\subsubsection{Interactions at BNNT-Molten Al Interface}

BNNTs were found to survive high-temperature conditions involved in the melting of Al. A network of long nanotube clusters in the solidified Al matrix is shown in Figure 6.7a. A low magnification TEM image (Figure 6.7b) shows the presence of fine nanotubes in cast Al-BNNT composite. XRD characterization of the composite pellet was performed to identify the phases. Peaks corresponding to h-BN were detected, as shown in Figure 6.7c. Despite high chemical inertness of BNNT, some interfacial reactions were noticed, as evidenced by the XRD pattern. Peaks corresponding to $\mathrm{AlN}, \mathrm{AlB}_{2}$, and $\mathrm{AlB}_{10}$ are shown in Figure 6.7.

The reactions and phenomena at the interface were examined by HR-TEM investigations. Figure 6.8a shows the formation of $\mathrm{AlN}$ due to the reaction between $\mathrm{Al}$ and h-BN. Regions corresponding to the $\mathrm{Al}$ matrix, $\mathrm{AlN}$ and $\mathrm{Al} / \mathrm{AlN}$ interface are clearly shown. Unlike the earlier report on solid-state reactions between $\mathrm{Al}$ and $\mathrm{BNNT}$ where $\mathrm{AlN}$ was discontinuously formed ${ }^{3}$, here we see a continuous and homogeneous formation of the product along the nanotube boundary. This is clearly shown in Figure $\mathbf{6 . 8 b}$, where AlN is seen to be formed along the entire length of BNNT as a film. $\mathrm{AlB}_{2}$ was not detected by TEM. Lahiri and co-workers ${ }^{3}$ thermodynamically calculated the formation of reaction products and showed that the volume of $\mathrm{AlN}$ formed is 1.55 times more than that of $\mathrm{AlB}_{2}$. It was shown that the formation of $\mathrm{AlN}$ is more feasible compared to $\mathrm{AlB}_{2}$ due to more negative Gibbs free energy. It was also observed that BNNT is mostly covered by AlN, with $\mathrm{AlB}_{2}$ embedded at few locations. Possibly the regions captured in TEM micrographs here did not have the $\mathrm{AlB}_{2}$ phase. The AlN layer formed on the BNNT wall is very thin $\sim 4-5 \mathrm{~nm}$. 

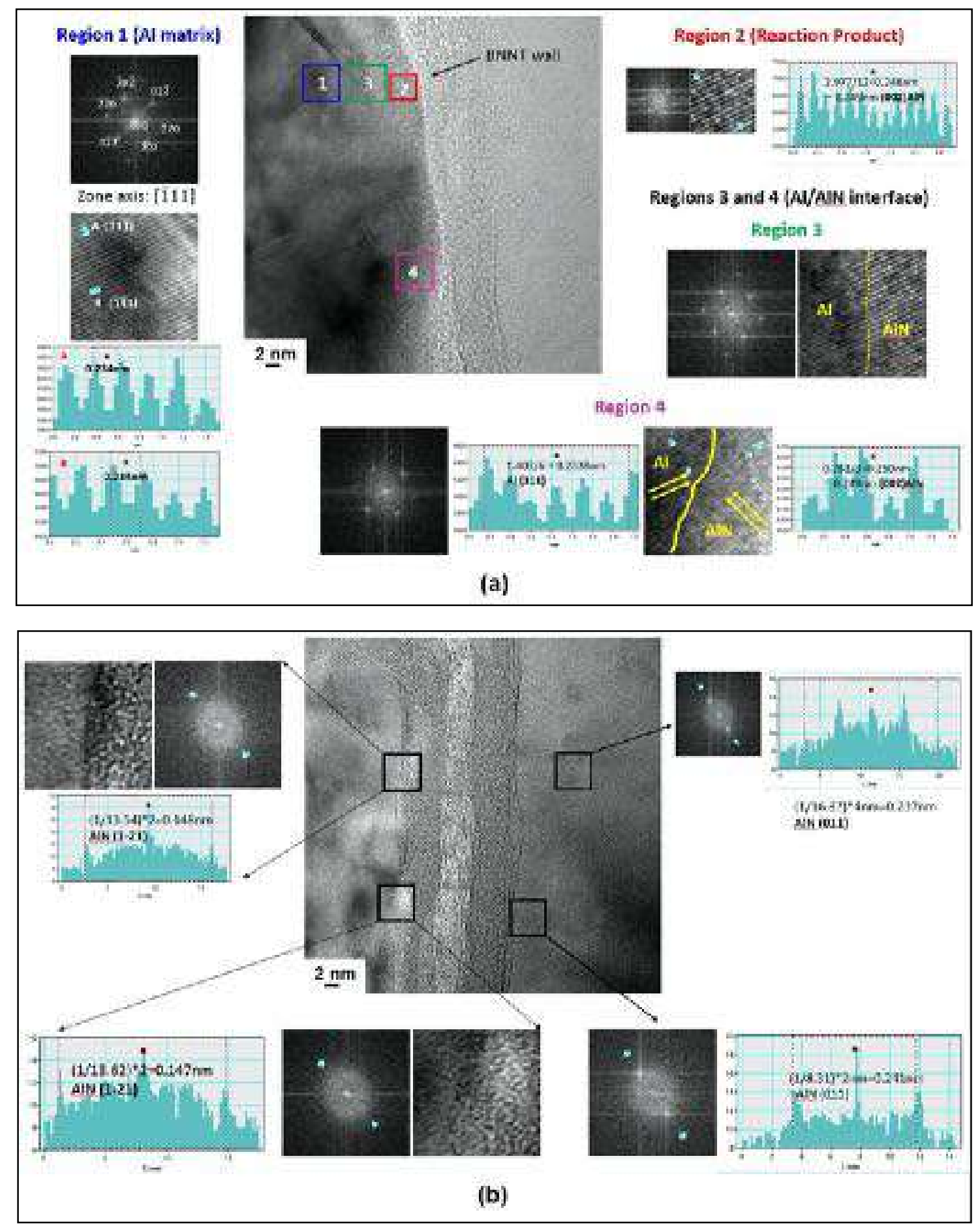

Figure 6.8. High resolution TEM and lattice images showing: (a) formation of AlN at AlBNNT interface, and (b) complete coverage of nanotube walls by AlN. 


\subsubsection{Reactive Wetting of BNNT by Molten Al}

Wettability of a solid surface by a liquid is determined by the equilibrium contact angle, which is related to interfacial tensions by the relation:

$$
\cos \theta=\frac{\gamma_{s}-\gamma_{s / l}}{\gamma_{l}}
$$

where $\gamma_{\mathrm{s}}$ is the surface energy of the solid phase being wetted, $\gamma_{1}$ is the surface tension of the liquid, and $\gamma_{\mathrm{s} / 1}$ is the solid-liquid interfacial tension. Based on Eq. (6.5), a high value of surface energy $\left(\gamma_{\mathrm{s}}\right)$ is desired for better wetting. The reported surface energy of BNNT is quite low $\sim 27 \mathrm{mN} / \mathrm{m}^{11}$, which would result in higher contact angle and hence poor wetting. The reported values of equilibrium contact angle for molten $\mathrm{Al}$ on h-BN vary from 140$160^{\circ 12,13}$, suggesting poor interfacial wetting and adhesion. As evidenced by HRTEM (Figure 6.8), the formation of reaction products takes place at the Al/BNNT interface. Therefore, the interfacial chemical reaction would alter the wetting behavior of the AlBNNT system. Since the AIN phase is the predominant product formed along the nanotube walls, the surface energies associated with AlN would play a key role in determining the wetting of nanotube by molten Al. AlN is reported to have a surface tension of about 660 $\mathrm{mN} / \mathrm{m}^{14}$, which is $\sim 24$ times larger than $\mathrm{BNNT}^{11}$. Based on Eq. (6.5), molten $\mathrm{Al}$ would favorably wet the AlN layer formed on BNNT. The reported stationary contact angles at the Al-AlN interface are in the range of $40-60^{\circ} \mathrm{C}$ in most of the studies ${ }^{15,16}$. Contact angle $<90^{\circ}$ signifies the good wettability of AlN coated nanotube. There are reports in the literature that attest that the formation of $\mathrm{AlN}$ at the $\mathrm{Al} / \mathrm{BN}$ interface promotes wetting ${ }^{17,18}$. The phenomenon of AlN induced interfacial wetting is schematically shown in Figure 6.9. 

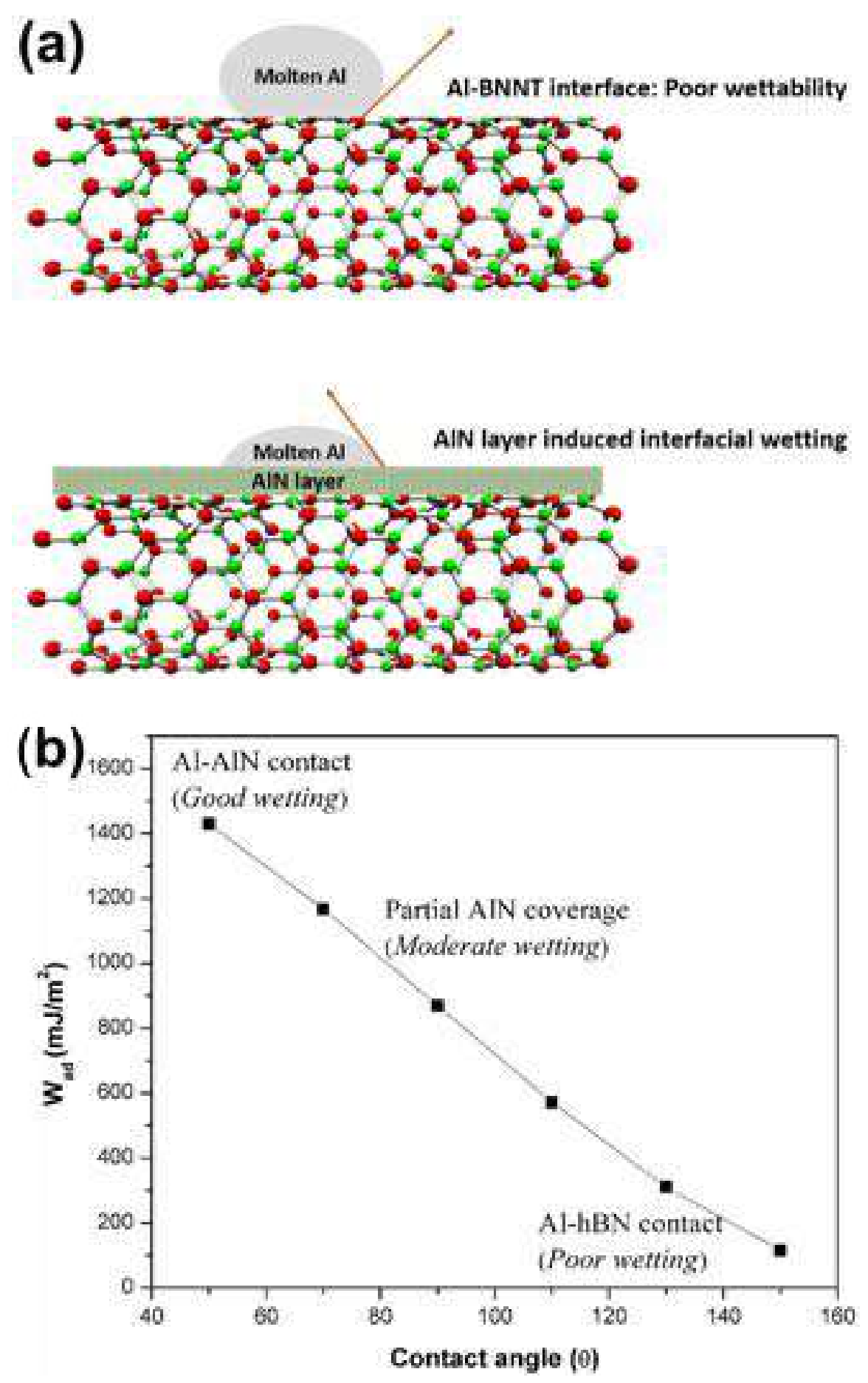

Figure 6.9. (a) Schematic representation of AlN induced wetting of BNNT by molten Al, and (b) theoretical calculations for the work of adhesion as a function of the extent of AlN formation at Al-BNNT interface. 


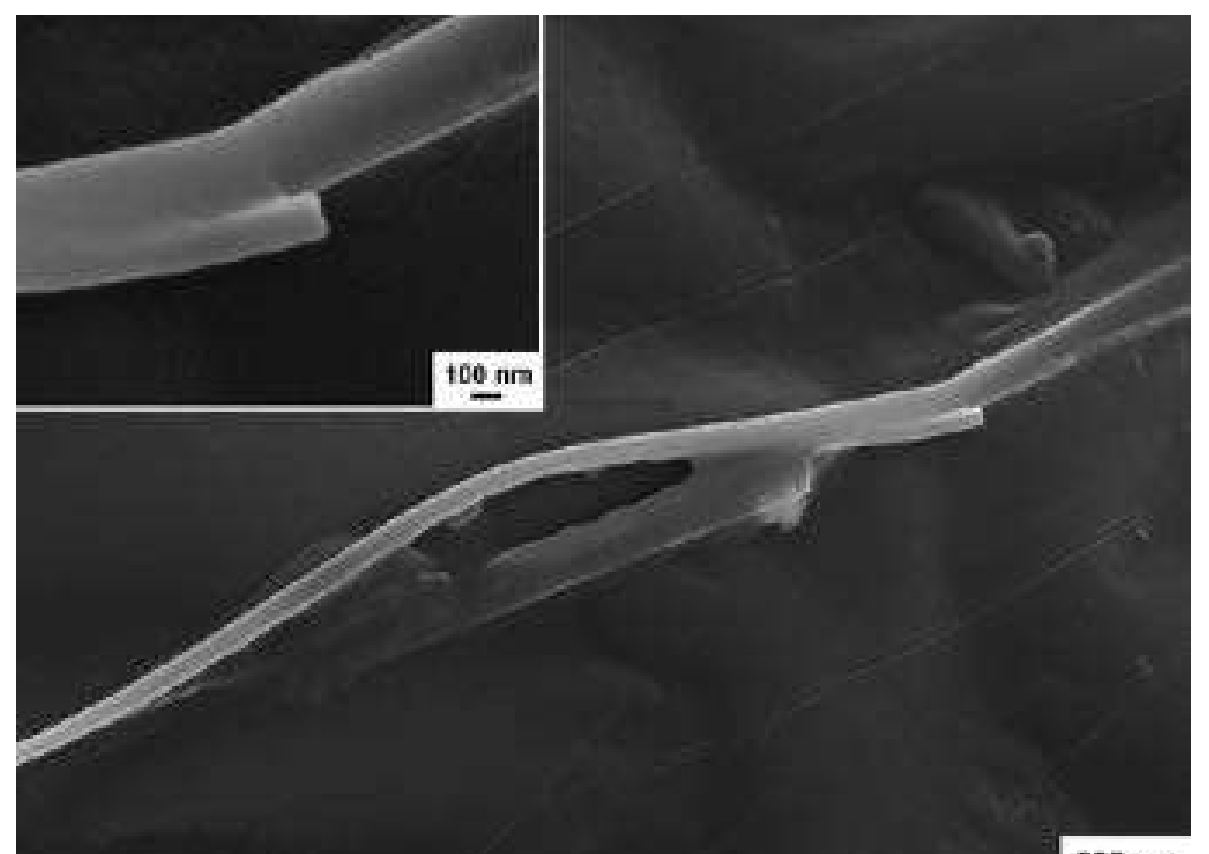

$500 \mathrm{~nm}$

Figure 6.10. SEM image showing BNNT completely wrapped by molten Al, confirming excellent interfacial wetting. Inset shows a high magnification image of nanotubes uniformly coated by $\mathrm{Al}$.

Energetics of interface in a composite system is characterized by two important thermodynamic parameters: work of adhesion $\left(\mathrm{W}_{\mathrm{ad}}\right)$ and interfacial energy $(\gamma)$. The work of adhesion is defined as the reversible work needed to separate an interface into two free surfaces. Therefore, the difference between final (two separate surfaces) and initial (bonded surfaces) energy states would give the adhesion work ${ }^{19-21}$ :

$$
W_{a d}=\frac{E_{A}+E_{B}-E_{A / B}}{A_{i}}
$$

where $E_{A}$ and $E_{B}$ are free surface energies of the two components of a composite system, $E_{A / B}$ is the interfacial energy and $A_{i}$ is the interfacial contact area. This equation can, 
therefore, be expressed in terms of surface energy. Since the system studied here comprises of liquid-solid interaction, the equation can be written as:

$$
W_{a d}=\gamma_{s}+\gamma_{l}-\gamma_{s / l}
$$

Work of adhesion in Al-BNNT composite system is related to the contact angle made by molten $\mathrm{Al}$ on the solid surface (i.e. BNNT/AlN), and on the surface tension of molten Al by the relation:

$$
W_{a d}=\gamma_{l}(1+\cos \theta)
$$

The surface tension of molten $\mathrm{Al}$ is temperature-dependent, and is expressed as ${ }^{22}$ :

$$
\gamma_{A l}=1043-0.18 T(\mathrm{mN} / \mathrm{m})
$$

At $700^{\circ} \mathrm{C}, \gamma_{A l}$ (which is $\gamma_{l}$ ) would be $\sim 870 \mathrm{mN} / \mathrm{m}$ (based on Eq. (6.9) above). An earlier study on Al-BNNT solid-state reaction showed that the kinetics of interfacial reaction product formation is very slow, due to the high chemical inertness of BNNT ${ }^{3}$. Therefore, AlN would not be covering the entire nanotube wall in the beginning, but would slowly form with time. The interfacial contact would progressively transition from $\mathrm{hBN}$ $\mathrm{Al}$ interactions to $\mathrm{AlN}-\mathrm{Al}$ interactions. Hence, the effective interfacial contact angle is expected to drop from $\sim 150^{\circ}$ (for $\mathrm{hBN}-\mathrm{Al}$ interface) to $\sim 50^{\circ}$ (for $\mathrm{AlN}-\mathrm{Al}$ interface) $[37,38,40-42]^{12,13,15,16}$. Due to this reactive wetting, the work of adhesion would increase from $117 \mathrm{~mJ} / \mathrm{m}^{2}$ for $\theta=150^{\circ}$ to $1430 \mathrm{~mJ} / \mathrm{m}^{2}$ for $\theta=50^{\circ}$ (Figure 6.9b). An increase in the work of adhesion implies that the amount of reversible work to separate $\mathrm{Al}$ and $\mathrm{AlN}$ is higher than $\mathrm{Al}$ and h-BN. In other words, the formation of AlN results in a stronger interface. 

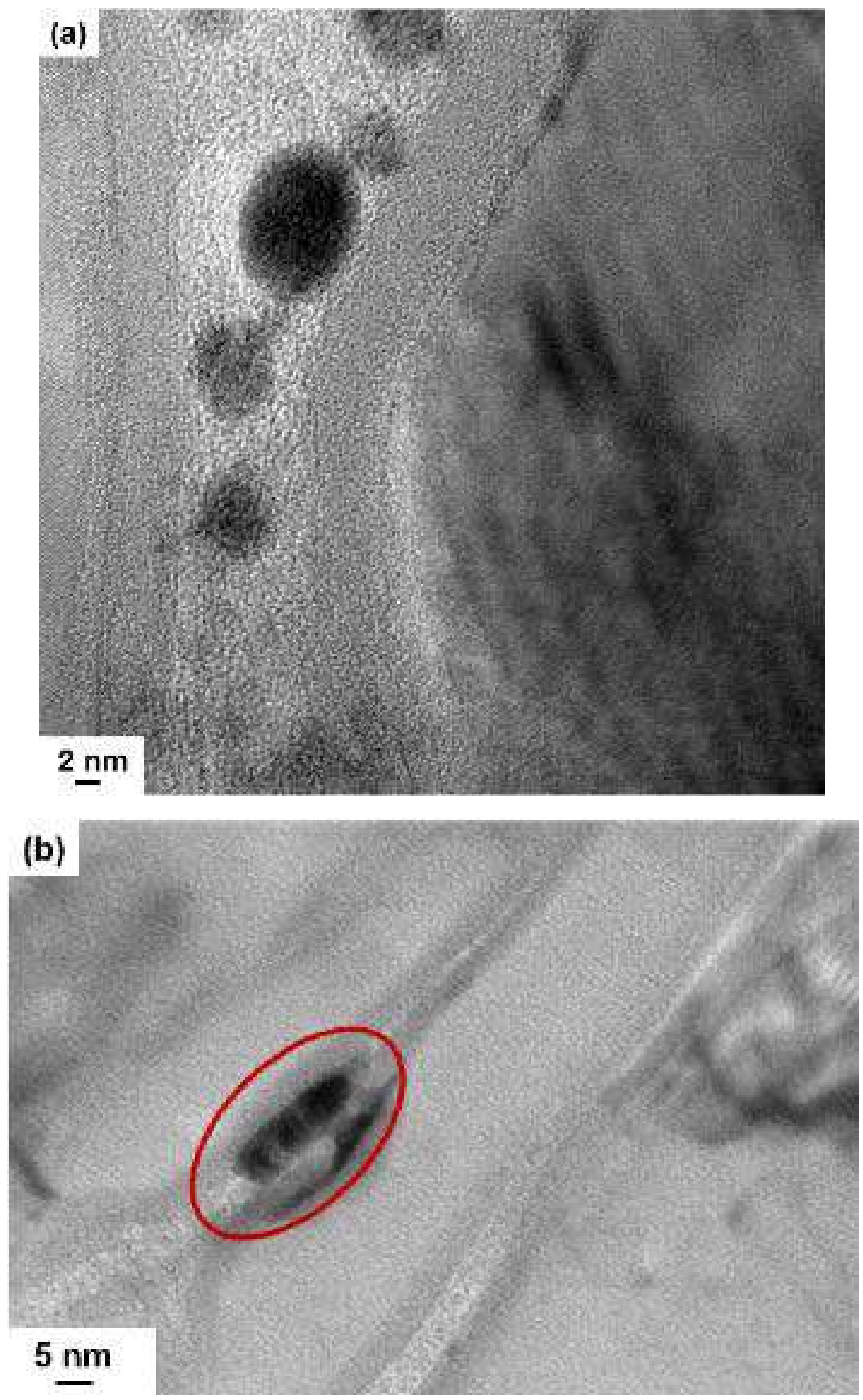

Figure 6.11. TEM images showing filling of BNNTs: (a) moderate capillarity represented by nano-droplets, and (b) strong capillary effect resulting in filling by larger rod-shaped solid. 
Based on Eq. (6.7), we obtain the Al/BNNT interfacial energy ( $\left.\gamma_{\mathrm{BNNT} / \mathrm{Al}}\right)$ to be $\sim 780$ $\mathrm{mJ} / \mathrm{m}^{2}$ (assuming $\gamma_{\mathrm{s}}=\gamma_{\mathrm{BNNT}}=27 \mathrm{~mJ} / \mathrm{m}$ ), corresponding to molten Al-BNNT interface when there is no formation of AlN. When AlN forms on the nanotube surface, the interfacial energy $\left(\gamma_{\mathrm{AlN} / \mathrm{Al}}\right)$ is obtained to be $\sim 100 \mathrm{~mJ} / \mathrm{m}^{2}$ (assuming $\gamma_{\mathrm{s}}=\gamma_{\mathrm{AlN}}=660 \mathrm{~mJ} / \mathrm{m}$ ). A drop in the value of interfacial energy suggests that the formation of the Al/AlN interface is thermodynamically favored. Reactive wetting of BNNT by molten Al results in improved metal matrix-nanotube interface. This is evident from the SEM image in Figure 6.10 showing nanotube fibrils uniformly coated by Al. While the phenomenon of reactive

wetting is well understood in a metal-ceramic system ${ }^{23-25}$, we demonstrate in this study a chemical reaction induced wetting at the nanoscale where the interfacial interactions can be much more complex. At the same time, it is important to note that the AlN formation is desirable only in trace amounts for effective stress transfer from the Al matrix to BNNT. Excess reactions would compromise the structure and properties of BNNT. Therefore, the interfacial reaction should be controlled for superior properties.

\subsubsection{Reactive Filling of BNNT by Molten Al}

Due to their hollow anatomy, the ultra-fine nanotubes can exhibit capillarity. Filling of BNNTs by metallic nanoparticles and nanowires like Fe, Ni, Fe-Ni, Mg, Co, Au, Pt, Pd, $\mathrm{Ag}$ and $\mathrm{In}^{26-32}$, molybdenum clusters ${ }^{33}$, crystals of potassium halides like $\mathrm{KCl}, \mathrm{KBr}$ and $\mathrm{KI}^{34}$, fullerene $\mathrm{C}_{60}{ }^{35}, \mathrm{CNTs}^{36}, \mathrm{ZrO}_{2}{ }^{37}, \mathrm{NiSi}_{2}{ }^{31}, \mathrm{MgO}^{32}$, boron carbide ${ }^{38}$, and $\mathrm{SiC}^{39}$ is reported in the literature by techniques such as wet chemistry, chemical vapor transport and in situ filling during BNNT synthesis. We report for the first time filling of BNNT 
immersed in a molten metal pool through capillarity. Capillary suction of molten Al is shown in Figures. 6.11a and 6.11b, where solidified particles can be seen inside BNNT.

Capillarity and wetting are inter-linked phenomena ${ }^{40}$. This is evident from the Laplace relation:

$$
\Delta P=\frac{2 \gamma_{l} \cos \theta}{r}
$$

where $\Delta P$ is the pressure difference across the liquid-vapor interface, $\theta$ is the contact angle, $\gamma_{l}$ is the surface tension of molten $\mathrm{Al}$ and $r$ is the nanotube diameter. This pressure difference is termed as Laplace pressure, which is the driving force for capillary action. From Eq. (6), $\theta>90^{\circ}$ would imply negative $\Delta \mathrm{P}$. Therefore, for capillary action to take place spontaneously, the contact angle should be less than $90^{\circ}$. Favorable wetting is essential for the capillary suction of molten Al by hollow BNNT. For contact angles greater than $90^{\circ}$, the pressure would have to be applied to fill the nanotubes with Al. These nanotubes are extremely fine ( $10 \mathrm{~nm}$ diameter) and the surface tension of molten $\mathrm{Al}$ at $700^{\circ} \mathrm{C}$ is also quite high $\sim 870 \mathrm{mN} / \mathrm{m}$. Therefore, for the h-BN/molten $\mathrm{Al}$ interface, $\Delta \mathrm{P}$ would have a large negative value of $\sim-300 \mathrm{MPa}$ (assuming the contact angle to be $\sim 150^{\circ}$ ). Therefore, BNNT is unlikely to exhibit capillarity. However, as AIN formation initiates and a final contact angle of $\sim 50^{\circ}$ is accomplished, $\Delta \mathrm{P}$ becomes positive with a value of 220 MPa which would result in capillary suction of Al. The variation of Laplace pressure as a function of contact angles is shown in Figure 6.12. Three different capillarity regimes are demarcated, with negative, moderately positive and highly positive Laplace pressures. These different regimes were observed in this study, which is linked to the variation in rate 
and extent of individual nanotube reactivity to form interfacial products. The reactivity and subsequent filling of individual nanotubes would vary based on the defect sites, clustering, length and diameter of the nanotubes. Because of this, both moderate as well as strong capillary effect were observed in TEM micrographs for different BNNTs. Solidified Al spherical nanoparticles inside BNNT shown in Figure 6.11a represent moderate capillarity associated with intermediate contact angles. On the other hand, larger rod-shaped solid filled inside the nanotube represents strong capillarity regime (Figure 6.11b). These regimes are schematically illustrated in Figure 6.12.

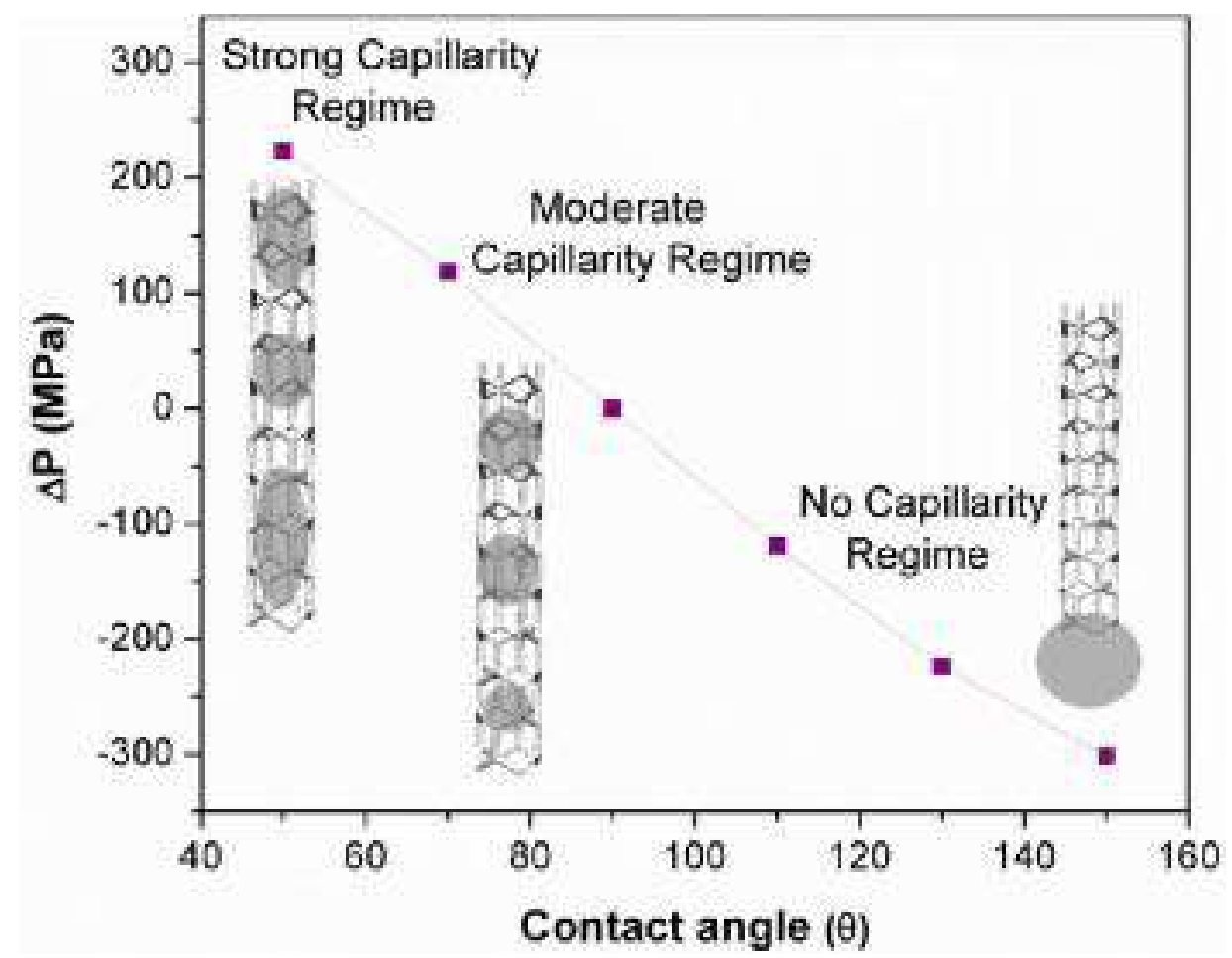

Figure 6.12. Variation of Laplace pressure with contact angle and the schematic representation of capillarity-induced filling.

An interesting feature from Figure 6.11a is the constriction of nanotube diameter. This can be related to the kinetics of capillary suction as the Al-BNNT reaction proceeds 
to form more AlN. Due to the progressive drop in contact angle with AlN formation on nanotube walls, the capillarity would also increase. As a result, for the rapid intake of molten $\mathrm{Al}$, the nanotube diameter starts constricting. This is explained by the equation of continuity for fluid in motion inside a tube, which states that the product of fluid velocity and cross-section area of the tube is constant:

$$
A_{1} V_{1}=A_{2} V_{2}
$$

Constriction of nanotube diameter would increase the velocity of molten Al being sucked inside the nanotube to satisfy Eq. (7). This enhanced velocity of suction results in a large intake of molten Al. The contraction of nanotubes, therefore, takes place to accommodate enhanced capillarity due to interfacial reaction. This also evidences the high structural flexibility of BNNT.

It is found that the Al sucked inside the nanotube reacts with the BNNT wall. The formation of $\mathrm{AlN}$ and $\mathrm{AlB}_{10}$ phases is revealed by lattice spacing measurements (Figure 6.13). This is quite an interesting observation, as the formation of $\mathrm{AlB}_{10}$ due to the AlBNNT reaction has hitherto not been reported. $\mathrm{AlB}_{10}$ has a tetragonal structure with a $\mathrm{Cmcm}$ space group. Unlike the exterior region, the volume of $\mathrm{Al}$ inside the nanotube is limited because of poor capillarity in the beginning, which tends to accelerate with time as AlN forms. As a result, the relative amount of $\mathrm{B}$ is higher than $\mathrm{Al}$ inside BNNT. This explains the preferential formation of the $\mathrm{B}$-rich $\mathrm{AlB}_{10}$ phase inside the nanotube due to the Al-BNNT reaction, contrary to the outer wall reaction where $\mathrm{AlB}_{2}$ forms due to sufficiently high Al concentration. 
While reactive infiltration of micron-sized pores is studied in past ${ }^{46,47}$, we report chemical reaction induced filling of a nanotube in this work. Capillary suction and subsequent reaction of the filled metal with the nanotubes will influence the mechanical behavior of the composite. Formation of ceramic phases inside the nanotubes will enhance the elastic modulus and strength of the composite (ceramics are typically stronger than pure Aluminum), but at the same time may limit the ductility (due to inherent brittle nature of ceramics). Therefore, the processing conditions and processing time need to be controlled in accordance with the mechanical characteristics desired in the cast metal matrix composite.

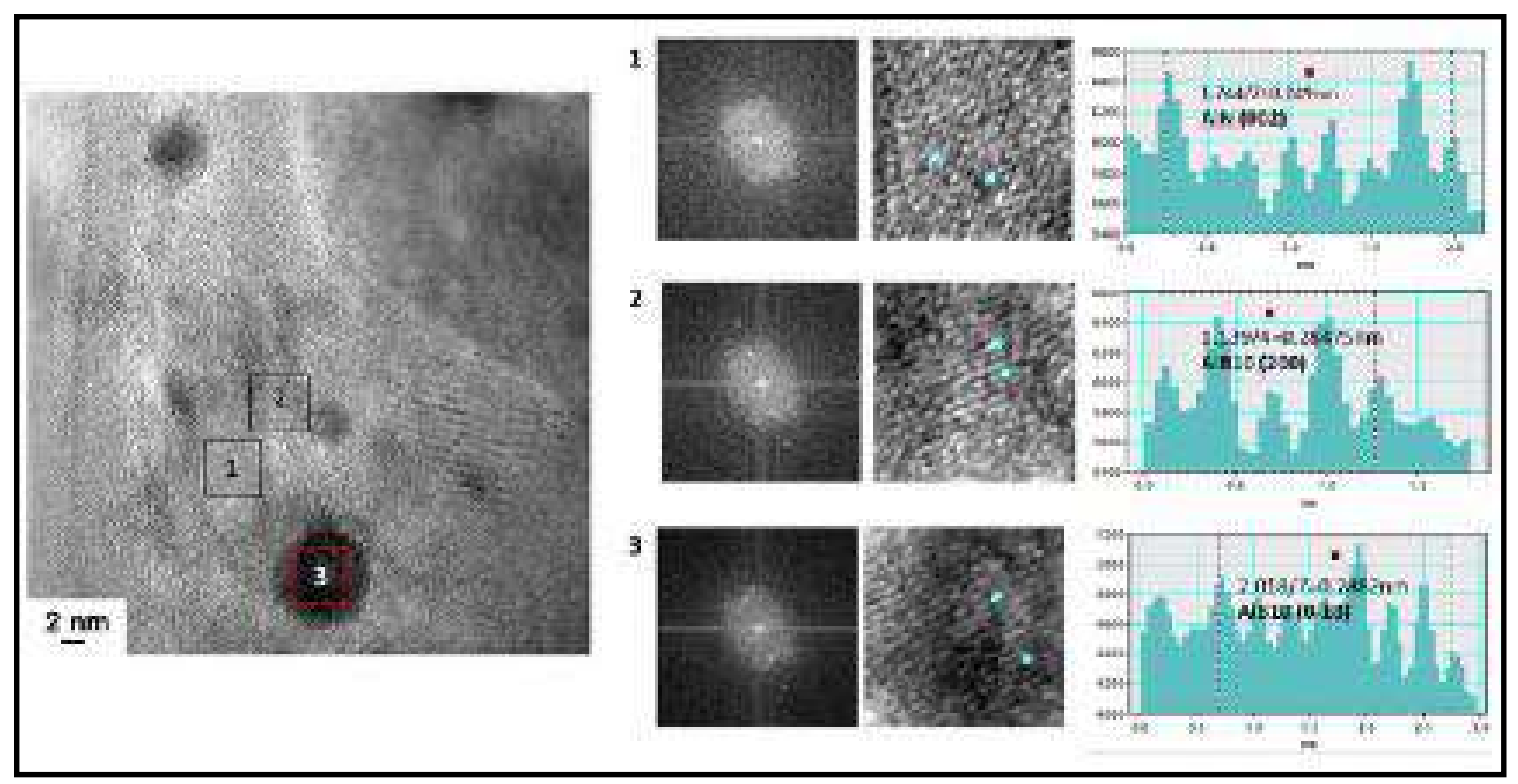

Figure 6.13. Analysis of reaction between BNNT and Al inside the nanotube.

\subsubsection{Mechanical Response}

The microstructure investigations discussed in the preceding sections revealed a highly hierarchical microstructure of Al-BNNT composites fabricated by solidification. The 
overall mechanical response of the composite will be influenced by the microstructure constituents, such as high aspect ratio nanotubes, interfacial reaction products, ductile Al matrix, as well as the interactions between these features. To assess the local, site-specific response from the microstructure, nanoindentation investigations were performed. As expected, there was a prominent scatter or inhomogeneity in the force-displacement response (Figure 6.14). The corresponding elastic modulus and hardness are included in Table 6.1. Indent 1 characterizes typical Al metal response, with elastic modulus and hardness values of $\sim 70 \mathrm{GPa}$ and $\sim 700 \mathrm{MPa}$, respectively. On the other end of the spectrum, Indent 4 demonstrates extraordinary hardening and stiffening with over $120 \%$ enhancement in modulus and over $1400 \%$ jump in nanohardness. This response captures the effect of BNNTs and hard ceramic phases in the microstructure. The intermediate modulus and hardness represent the response from the elastic-plastic zone comprising of a combination of BNNT, $\mathrm{Al}$ and/or interphases. These findings confirm that BNNT retains its reinforcement potential after solidification and extreme thermal conditions involved in casting do not seem to have any detrimental effect on its mechanical characteristics. Therefore, reactive interface bonding is promising for mechanical reinforcement of lightweight Al metal.

Table 6.1. Microstructure-sensitive mechanical signature from as-solidified Al-BNNT composite.

\begin{tabular}{lllll}
\hline & Indent 1 & Indent 2 & Indent 3 & Indent 4 \\
\hline $\mathbf{E}(\mathbf{G P a})$ & 70.43 & 113.8 & 126.3 & 158.35 \\
\hline H (GPa) & 0.69 & 0.92 & 2.5 & 10.86 \\
\hline
\end{tabular}




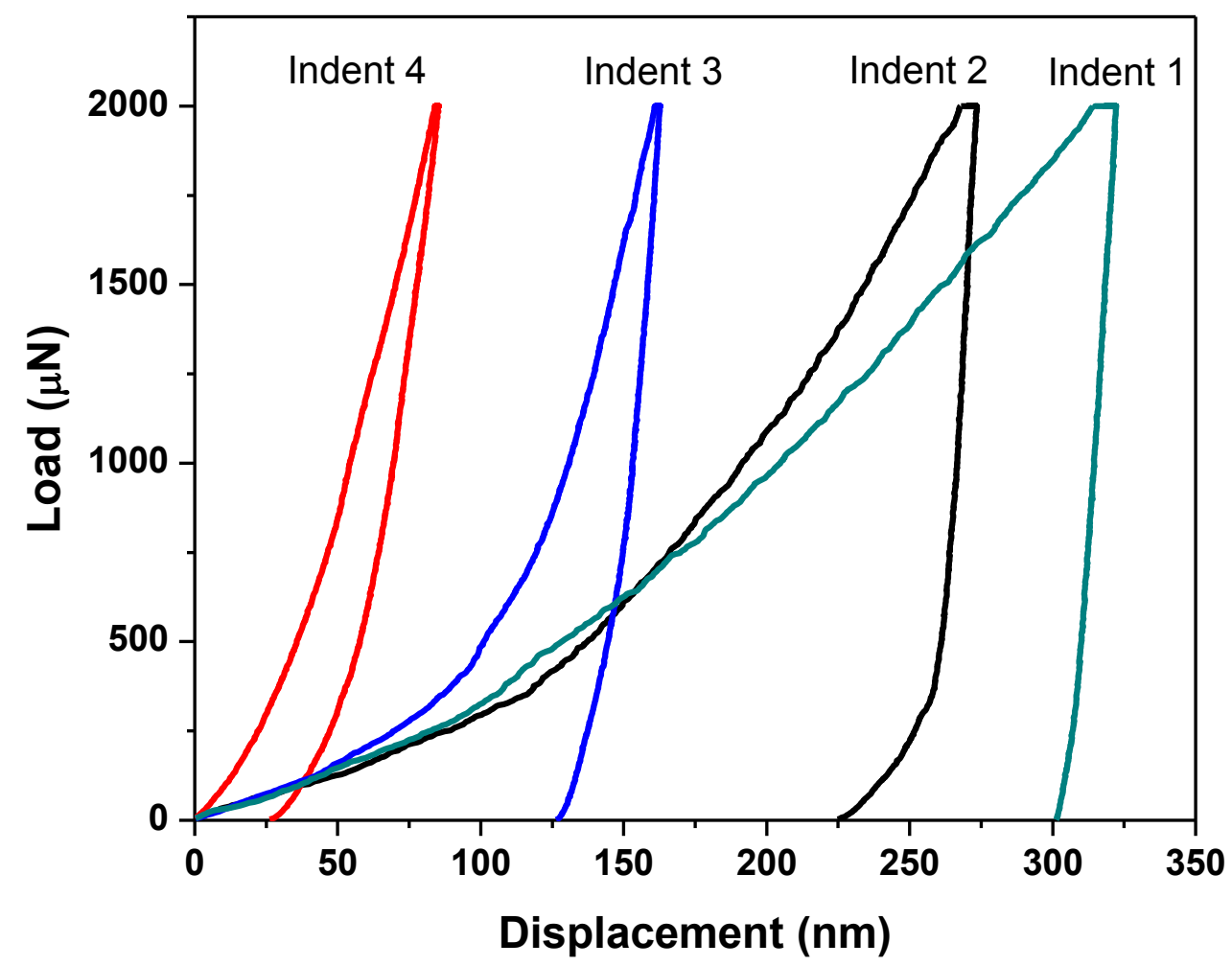

Figure 6.14. Microstructure-sensitive local response captured by nanoindentation of the as-solidified Al-BNNT composite.

\subsection{Layer-by-Layer Deposition of Al-BNNT by Plasma Spray}

\subsubsection{Survival and Integration of BNNT}

The obtained composite coating was $\sim 300 \mu \mathrm{m}$ in thickness and had $1 \mathrm{vol} . \%$ of BNNT. The plasma-sprayed deposit was sectioned, and the cross-section was metallographically polished. SEM micrograph of the polished cross-section revealed swirly features, which are Al splats (Figure 6.15a). Figure 6.15b shows the solidified microstructure, comprising of fused metal particles, some degree of porosity and tubular features. The nanotubes in the microstructure act as crack bridges during fracturing of the composite. The high magnification SEM micrograph shows the nanotube is well-embedded and wrapped by $\mathrm{Al}$ 
post-solidification (Figure 6.15c). X-ray diffraction (XRD) of the coating detected h-BN, $\mathrm{AlN}$ and $\mathrm{AlB}_{2}$ phases (shown in the inset of Figure 6.15d). This suggests the integration of the h-BN phase (associated with BNNT) in Al during plasma spray, along with minimal interfacial reactions. It should be noted that there was no prominent peak associated with $\mathrm{Al}_{2} \mathrm{O}_{3}$ detected in $\mathrm{XRD}$, indicating there is no pronounced oxidation during plasma spray.
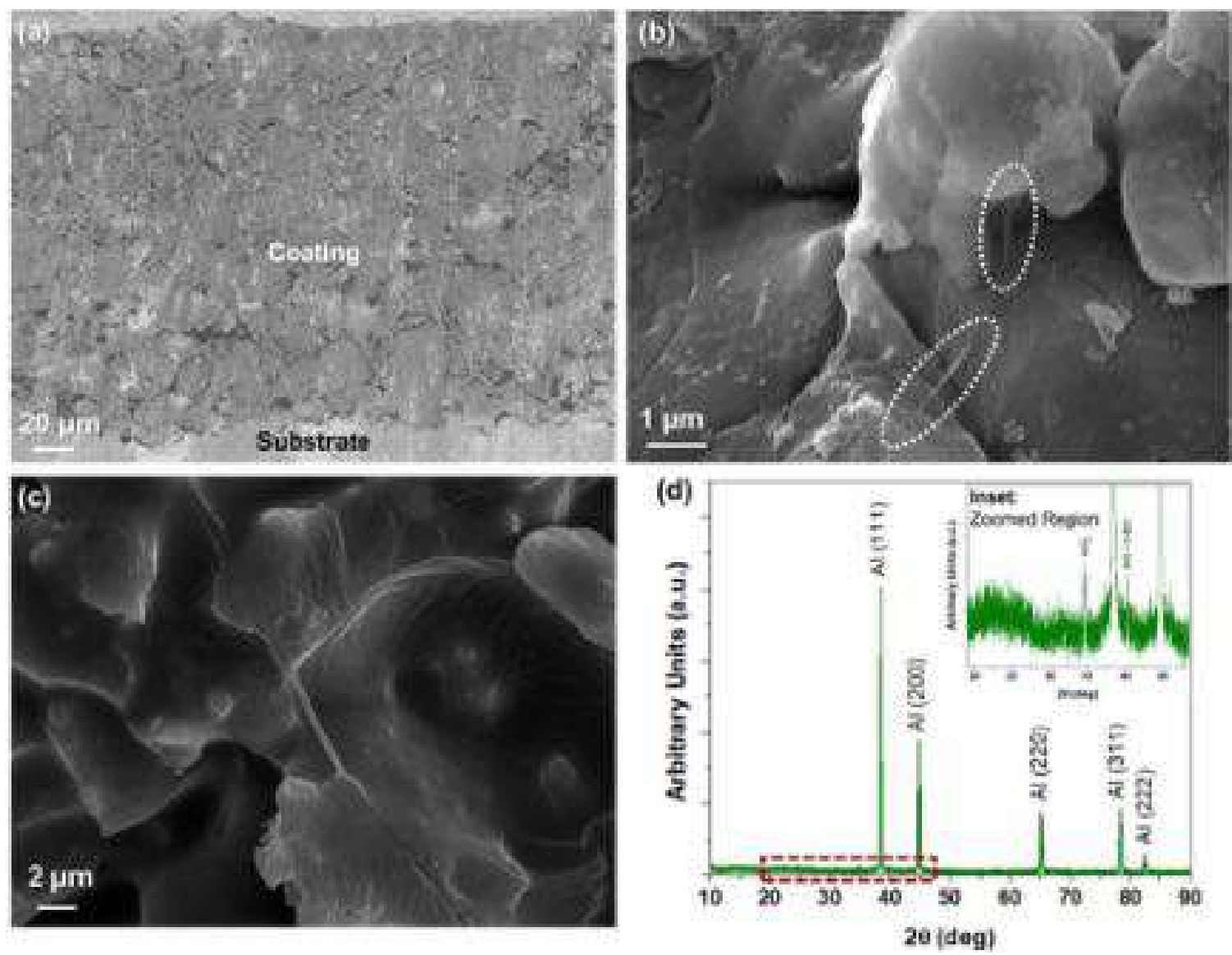

Figure 6.15. (a) SEM micrograph of the polished cross-section of plasma sprayed coating, showing swirly splats as the fundamental microstructural building blocks, (b) SEM micrograph showing long nanotubes in the microstructure, (c) high magnification SEM micrograph showing excellent wetting of BNNT during plasma spray, and (d) X-ray diffraction characterization of plasma-sprayed deposit confirms retention of h-BN phase in $\mathrm{Al}$, along with minimal interfacial reactions. 

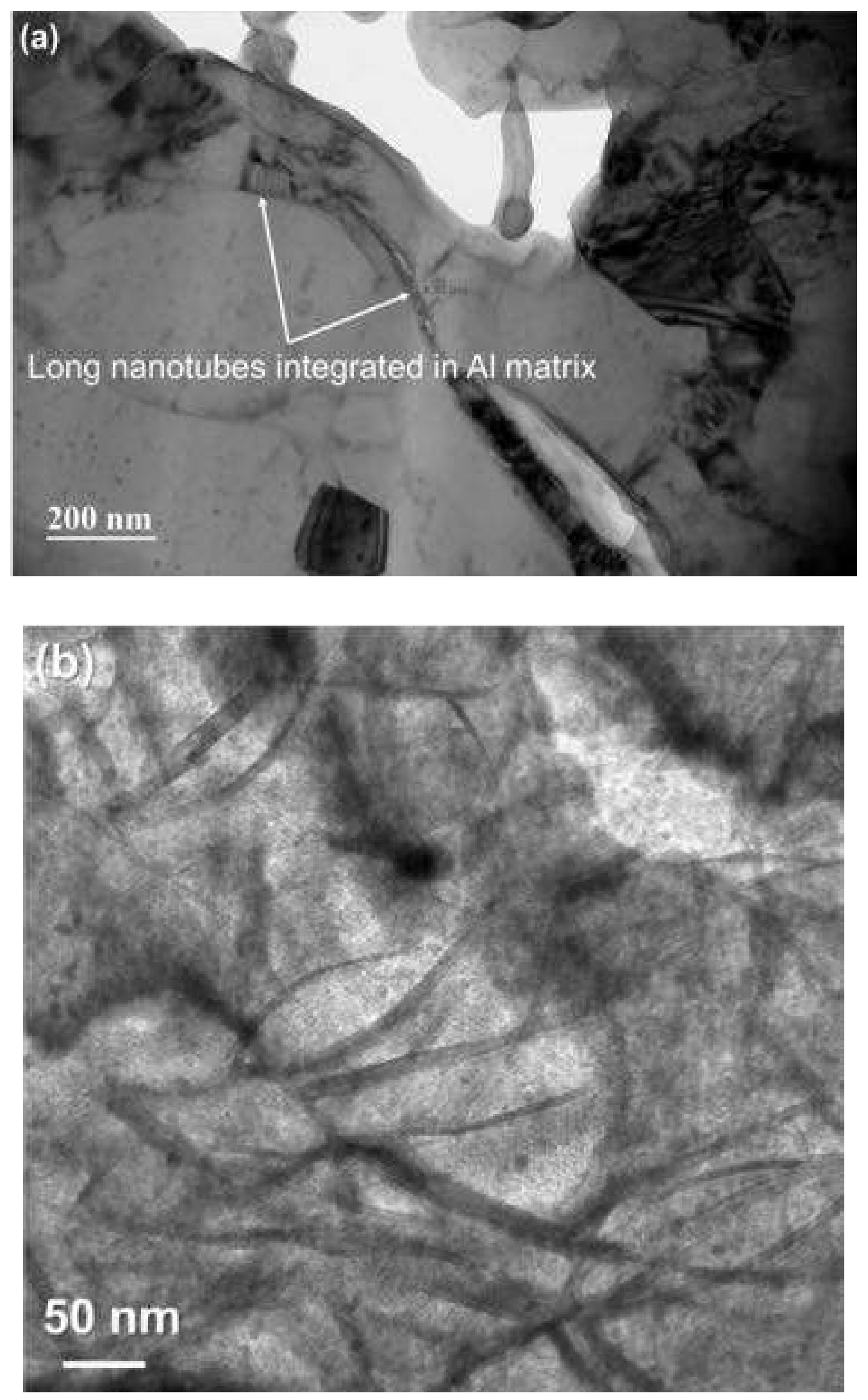


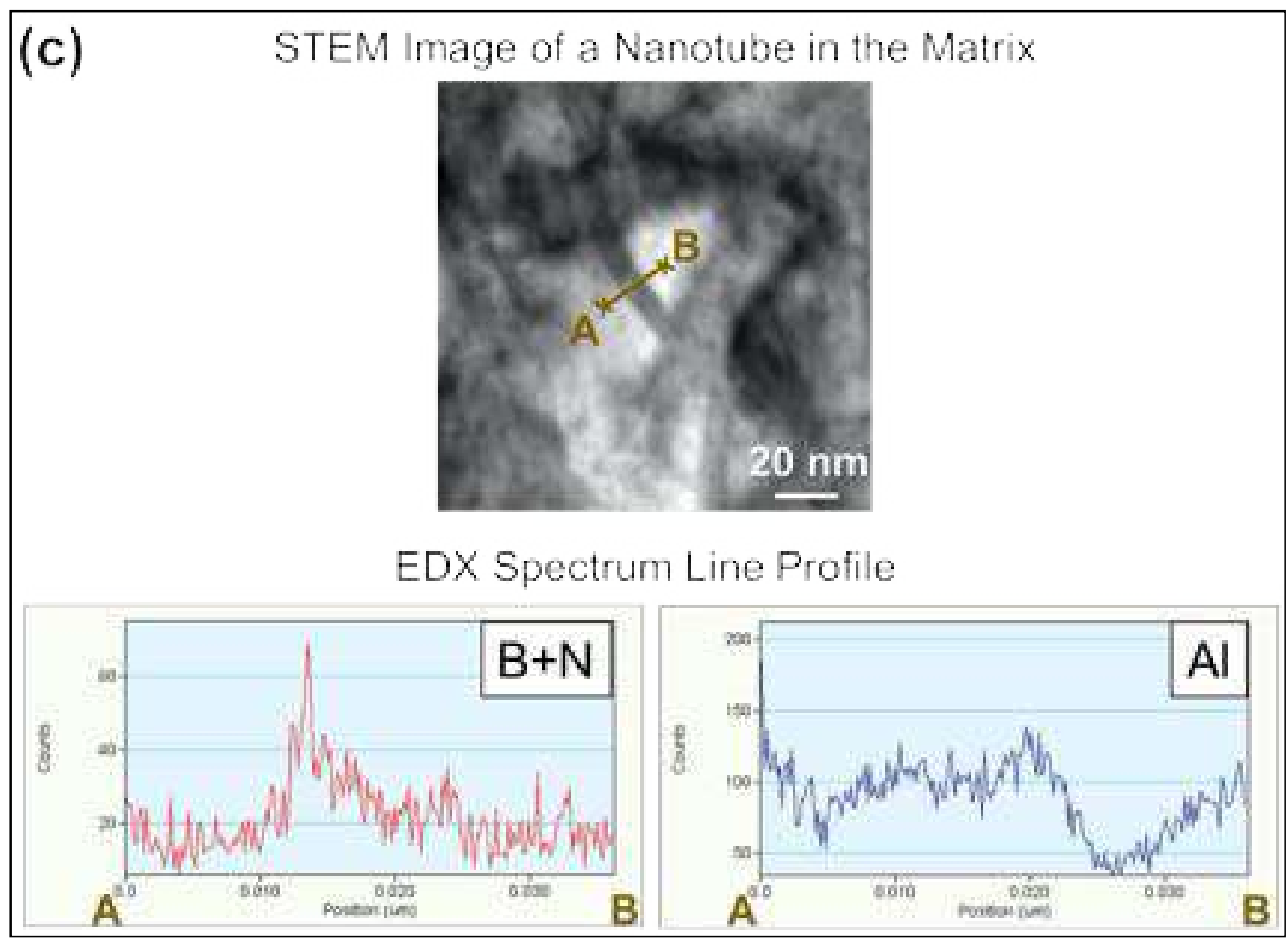

Figure 6.16. (a) Low magnification TEM micrograph showing integration of long nanotube in the matrix, (b) high magnification TEM micrograph showing a network of nanotubes in the matrix, and (c) STEM micrograph of the nanotube in the matrix and the corresponding EDX spectrum line profile confirming the survival of BN nanotube during plasma spray.

Transmission electron microscopy (TEM) was performed to gain insights into the microstructure evolution at Al/BNNT interface after plasma spray. Specimens for TEM were prepared using Focused Ion Beam machining (dual beam JEOL JIB-4500 focused ion beam/SEM, Tokyo, Japan). TEM investigation showed that the long nanotubes are integrated into the matrix (Figure 6.16a). A high magnification TEM revealed a network of multiple nanotubes forming a mesh in the composite microstructure (Figure 6.16b). It is evident from this micrograph that the chemical dispersion of nanotubes before plasma 
spray overcomes agglomeration and prevents clustering in the composite microstructure.

To analyze the chemical composition of the nanotubes in the matrix, scanning tunneling microscopy (STEM) was performed, and spectroscopic maps based on energy dispersive X-ray spectroscopy (EDX) were obtained. Figure 6.16c shows the STEM image of a region with a nanotube and the corresponding EDX line profile across the nanotube (line $\mathrm{AB}$ shown in the figure). $\mathrm{B}$ and $\mathrm{N}$ elements were detected along with $\mathrm{Al}$ during EDX. The line profile shows a spike/ peak in the $\mathrm{B}+\mathrm{N}$ profile corresponding to the nanotube region in the STEM image. The STEM/EDX study confirms that BNNT survives hightemperature conditions involved in plasma spray, and is efficiently integrated into Al to form a nanocomposite.

\subsubsection{Interfacial Interactions in Plasma}

The interfacial reactions between BNNT and Al during plasma spray were examined by high-resolution transmission electron microscopy. It was observed that the majority of the interface did not undergo a chemical reaction, but there were selective regions where $\mathrm{Al}$ and BNNT were found to react. Fast and inverse fast Fourier transform (FTT \& IFTT) analysis revealed traces of $\mathrm{AlB}_{2}$ and $\mathrm{AlN}$ along $\mathrm{Al} / \mathrm{BNNT}$ interface, as shown in Figure 6.17a. Selective area electron diffraction (SAED) was also performed on the nanotube. The pattern confirmed the presence of AIN on the nanotubes (Figure 6.17b). It is noteworthy that low chemical reactivity of $\mathrm{BNNT}^{3,41}$ and rapid solidification during plasma spray ${ }^{42}$ restricts the extent of reaction, resulting in localized product formation. This is in striking contrast to the equilibrium solidification process, where a continuous thin layer of AlN (4- 
$5 \mathrm{~nm}$ ) was formed throughout the length of the BNNT wall (Section 6.2.1). The trace amount of reaction products enhances the interfacial adhesion between the metal matrix and BNNT due to the formation of strong covalent bonds.

Effective capture of BNNT during solidification is a key challenge in the fabrication of metal matrix nanocomposites. Thermodynamically, the Gibbs free energy change associated with nanotube capture during solidification is expressed as ${ }^{43}$ :

$$
\Delta G=\pi r^{2} l\left(\sigma_{i S}-\sigma_{i L}\right)
$$

where $\sigma_{i s}$ and $\sigma_{i L}$ are the interfacial energies associated with $\mathrm{BNNT} /$ solidified $\mathrm{Al}$ interface and $\mathrm{BNNT} /$ molten $\mathrm{Al}$ interface, respectively. In the beginning, the interfacial contact takes place between $\mathrm{Al}$ and h-BN phase. Molten $\mathrm{Al}$ does not favorably wet $\mathrm{BN}$, with reported contact angles, $\theta>120^{\circ 13}$. During plasma spray, the interfacial chemical reaction takes place in selective regions, as revealed by TEM and SAED investigations (Figures 6.17a and b). It is reported that the contact angle made by molten $\mathrm{Al}$ with $\mathrm{AlN}$ is $<60^{\circ} 16,19$. Therefore, the formation of AlN during plasma spray improves the interfacial wetting, which results in the lowering of the interfacial energy, $\sigma_{i S}$ in the solidified composite, as compared to the beginning energy $\sigma_{i L}$. This will result in a negative $\Delta G$ (based on Eq. 6.12), suggesting that the nanotube capture during solidification is thermodynamically feasible. It is noteworthy that the total time of plasma exposure of particles is extremely short $(<1$ $\left.\mathrm{ms}^{44}\right)$ and the cooling rate is high $\left(10^{6}-10^{8} \mathrm{~K} / \mathrm{s}^{42}\right)$ in plasma spray. As a result, there are very limited interfacial reactions. Nevertheless, the even selective formation of reaction product will cause the lowering of Gibbs energy, attesting the thermodynamic feasibility of BNNT capture by Al. 

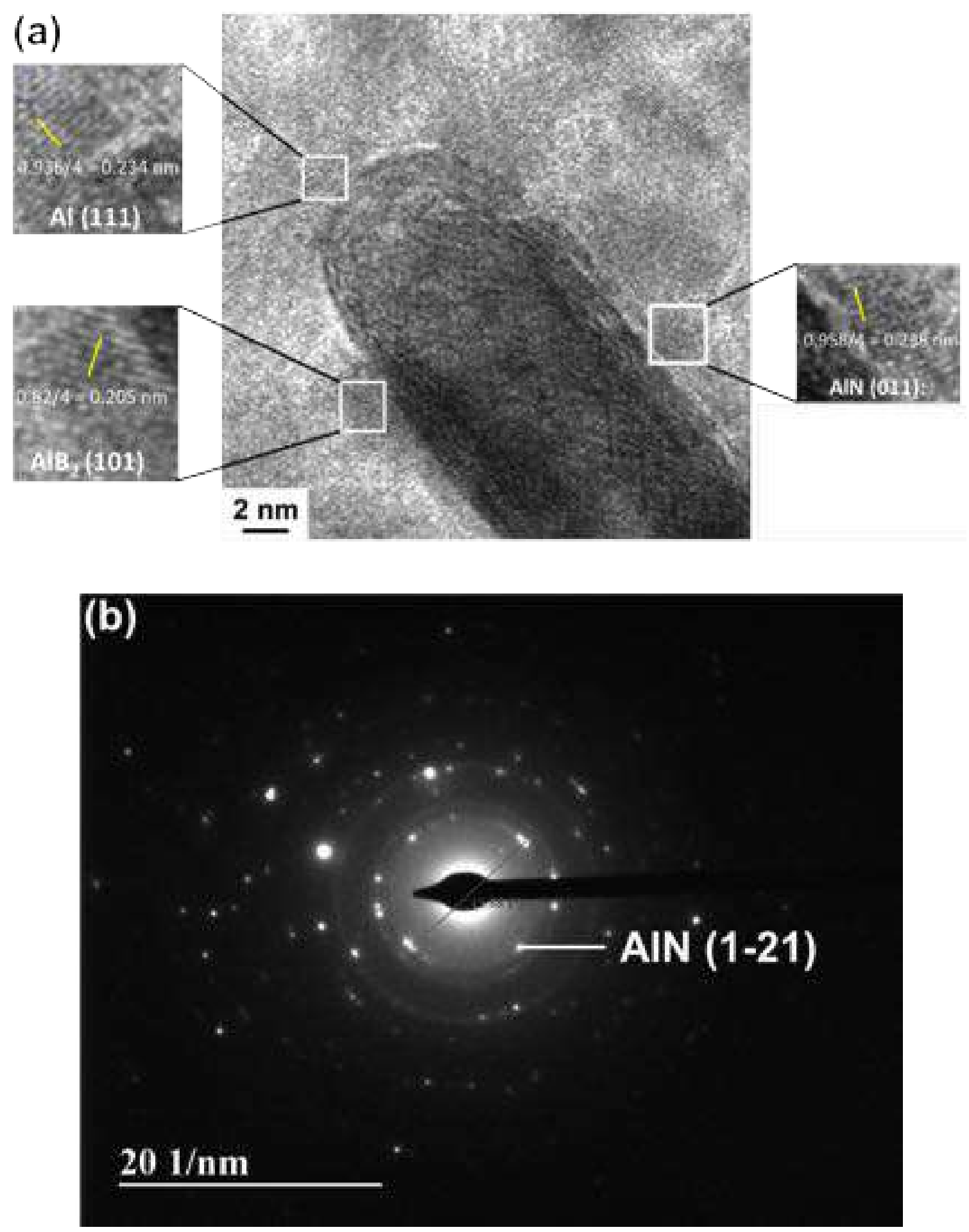

Figure 6.17. (a) HRTEM image and analysis showing reaction products at the A1/BNNT interface, and (b) SAED pattern obtained from a nanotube that confirms the formation of AlN during plasma spray. 
In addition to thermodynamic feasibility, overcoming the kinetic barrier to nanotube capture is a key challenge via solidification routes. The repulsive van der Waals forces tend to push the nanofillers as the solidification front advances. An opposing viscous drag due to molten metal slows down the pushing away of the nanoparticles. The velocity of the solidification front should be high enough to counter the van der Waals repulsion to be able to capture the BNNT in the melt. Theoretical calculations by Xu and co-workers show that critical velocity for capturing $\mathrm{Al}_{2} \mathrm{O}_{3}$ and $\mathrm{TiB}_{2}$ nanoparticles ( $20 \mathrm{~nm}$ in diameter) in $\mathrm{Al}$ is of the order of $10^{2}-10^{3} \mu \mathrm{m} / \mathrm{s}^{43}$. BNNT, with a diameter less than $6 \mathrm{~nm}$, will require much higher velocity for effective capture in Al. Unlike casting, plasma spray is a rapid solidification process, with particle residence time in plasma of the order of $10^{-3} \mathrm{~s}$. The solidification rate $(R)$ during plasma spray is obtained $b y^{45}$ :

$$
R=\frac{h\left(T_{t}-T_{S}\right)}{\rho L_{f}}
$$

where $h$ is the heat transfer coefficient, $T_{t}$ is the particle melting temperature, $T_{S}$ is the substrate temperature, $\rho$ is the particle density, and $L_{f}$ is the latent heat of fusion of the particle. The solidification rate for $\mathrm{Al}$ is calculated to be $\sim 1.5 \times 10^{5} \mu \mathrm{m} / \mathrm{s}^{45}$, which is at least 2 orders of magnitude higher than the critical, required velocity for capturing different nanoparticles in $\mathrm{Al}^{43}$. This attests to the feasibility of plasma spraying for efficiently integrating $\mathrm{BNNT}$ in $\mathrm{Al}$, and hence as a promising route for developing nanocomposites.

\subsubsection{Mechanical Reinforcement}

To ascertain the effect of BNNT addition on the mechanical properties of the plasma sprayed composite, 50 indents were made in different regions of the polished cross-section 


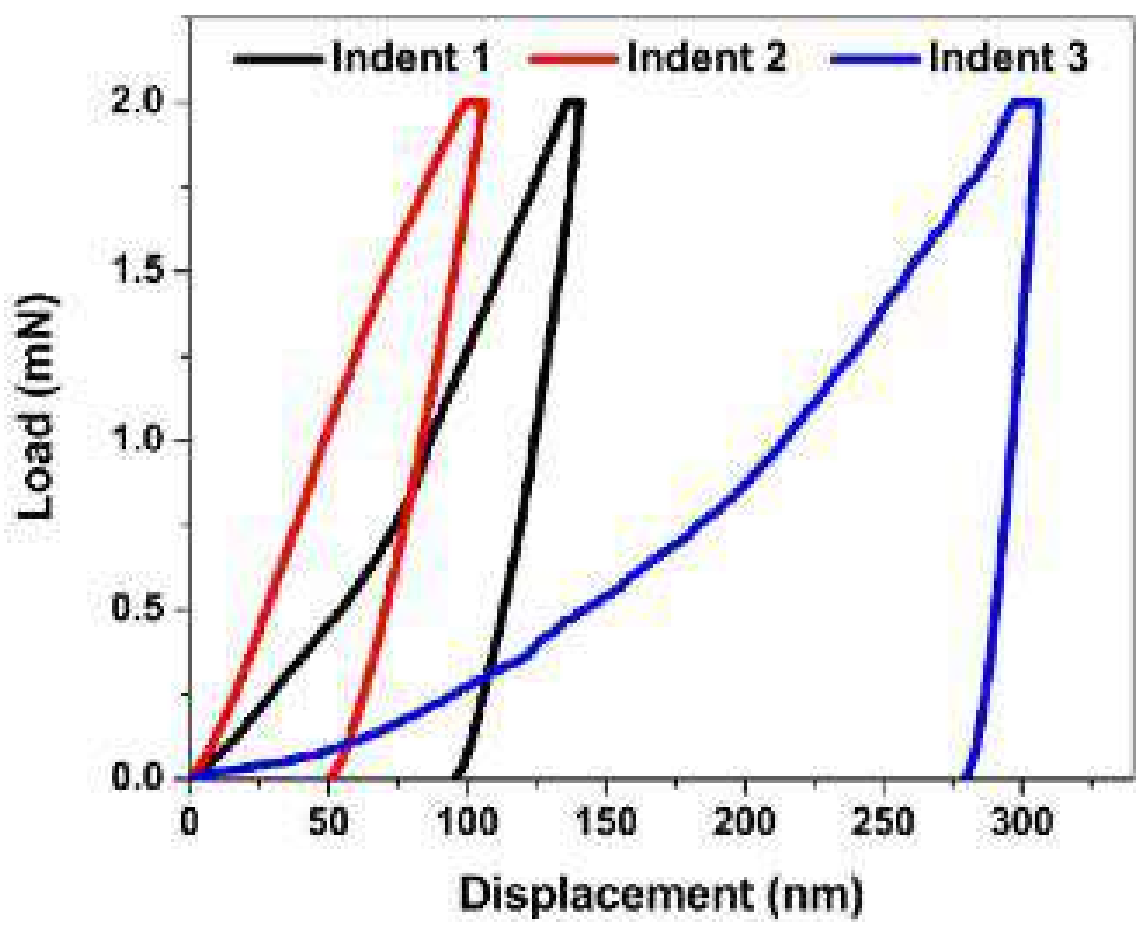

Figure 6.18. Nanoindentation load-displacement curves exhibiting the non-homogeneous mechanical response of the coating. Enhanced indentation-resistance in certain regions of the coating indicates BNNT reinforcement.

of the plasma sprayed Al-BNNT deposit. A heterogeneous mechanical response was obtained, as shown by three representative load-displacement curves shown in Figure 6.18. Indents 1 and 2 show higher resistance to plastic deformation, as compared to Indent 3. Indent 3 was found to exhibit modulus and hardness of $60.1 \mathrm{GPa}$ and $0.76 \mathrm{GPa}$, respectively, which are representative of the standard values for pure Al. About $80 \%$ of the indents were representative of Aluminum's properties. However, indents 1 and 2 demonstrate remarkably enhanced modulus and hardness: indent 1 corresponds to the elastic modulus and nanohardness of $87.73 \mathrm{GPa}$ and $8.61 \mathrm{GPa}$, respectively; and indent 2 is characterized by the modulus and hardness values of $83.57 \mathrm{GPa}$ and $3.83 \mathrm{GPa}$, respectively. About $20 \%$ of the indents made on the coating were characterized by higher 
modulus and hardness values. The localized enhancement in elastic modulus at BNNT-rich sites is $\sim 45 \%$ as compared to the $\mathrm{Al}$, whereas the jump in hardness is a whopping 10 times of pure $\mathrm{Al}$. These nanomechanical results evidence stiffening and hardening of $\mathrm{Al}$ due to BNNT and the ceramic reaction products formed during plasma spray.

\subsection{Summary Remarks}

Three different processing approaches were successfully employed to engineer Al-BNNT composites. The unique characteristics of each of these techniques were harnessed to design the MMC microstructure to augment the mechanical properties of lightweight Al.

The findings demonstrate the feasibility of employing widely accepted scalable manufacturing processes, with large volume processing capability, for developing lightweight and mechanically superior MMCs. This research provides critical insights for interface engineering in BNNT-MMCs and the reinforcement efficacy of integrated nanotubes.

\section{Output: Published Articles}

The contents of this chapter have been published as a series of journal articles on AlBNNT composites between 2016 and 2019:

(iii) P. Nautiyal, C. Rudolf, A. Loganathan, C. Zhang, B. Boesl, A. Agarwal, Directionally aligned ultra-long boron nitride nanotube induced strengthening of aluminum-based sandwich composite, Advanced Engineering Materials 18, 1747 (2016). 
(iv) P. Nautiyal, A. Gupta, S. Seal, B. Boesl, A. Agarwal, Reactive wetting and filling of boron nitride nanotubes by molten aluminum during equilibrium solidification, Acta Materialia 126, 124 (2017).

(v) P. Nautiyal, C. Zhang, B. Boesl, A. Agarwal, "Non-Equilibrium Wetting and Capture of Boron Nitride Nanotubes in Molten Aluminum During Plasma Spray”, Scripta Materialia 151, 71 (2018).

\section{References}

1. Lahiri, D. et al. Boron nitride nanotubes reinforced aluminum composites prepared by spark plasma sintering: Microstructure, mechanical properties and deformation behavior. Mater. Sci. Eng. A 574, 149-156 (2013).

2. Yamaguchi, M., Meng, F., Firestein, K., Tsuchiya, K. \& Golberg, D. Powder metallurgy routes toward aluminum boron nitride nanotube composites, their morphologies, structures and mechanical properties. Mater. Sci. Eng. A 604, 9-17 (2014).

3. Lahiri, D. et al. Insight into reactions and interface between boron nitride nanotube and aluminum. J. Mater. Res. 27, 2760-2770 (2012).

4. Polmear, I. Light alloys: metallurgy of the light metals. (Butterworth-Heinemann, 1995).

5. Kaufman, J. G. Properties of aluminum alloys: tensile, creep, and fatigue data at high and low temperatures. (The Aluminum Association, Washington DC and ASM International, 1999).

6. Wei, X., Wang, M.-S., Bando, Y. \& Golberg, D. Tensile tests on individual multiwalled boron nitride nanotubes. Adv. Mater. 22, 4895-4899 (2010).

7. Agarwal, A., Bakshi, S. R. \& Lahiri, D. Carbon Nanotubes: Reinforced Metal Matrix Composites. (CRC Press, Taylor \& Francis Group, 2011).

8. Kuzumaki, T., Miyazawa, K., Ichinose, H. \& Ito, K. Processing of Carbon Nanotube Reinforced Aluminum Composite. J. Mater. Res. 13, 2445-2449 (1998).

9. Chu, K., Wang, J., Liu, Y. \& Geng, Z. Graphene defect engineering for optimizing the interface and mechanical properties of graphene/copper composites. Carbon $N$. Y. 140, 112-123 (2018).

10. Ryu, H. J., Cha, S. I. \& Hong, S. H. Generalized shear-lag model for load transfer in SiC/Al metal-matrix composites. J. Mater. Res. 18, 2851-2858 (2003). 
11. Yum, K. \& Yu, M. F. Measurement of wetting properties of individual boron nitride nanotubes with the Wilhelmy method using a nanotube-based force sensor. Nano Lett. 6, 329-333 (2006).

12. Shen, P., Fujii, H. \& Nogi, K. Effect of temperature and surface roughness on the wettability of boron nitride by molten Al. J. Mater. Sci. 42, 3564-3568 (2007).

13. Xue, X. M., Wang, J. T. \& Quan, M. X. Wettability and spreading kinetics of liquid aluminium on boron nitride. J. Mater. Sci. 26, 6391-6395 (1991).

14. Martienssen, W. \& Warlimont, H. Springer handbook of condensed matter and materials data. (Springer, 2005).

15. Rosazza Prin, G., Baffie, T., Jeymond, M. \& Eustathopoulos, N. Contact angles and spreading kinetics of $\mathrm{Al}$ and Al-Cu alloys on sintered AlN. Mater. Sci. Eng. A 298, 34-43 (2001).

16. Naidich, Y. V. \& Taranets, N. Y. Wettability of aluminium nitride by tinaluminium melts. J. Mater. Sci. 33, 3993-3997 (1998).

17. Nicholas, M. G., Mortimer, D. A., Jones, L. M. \& Crispin, R. M. Some observations on the wetting and bonding of nitride ceramics. J. Mater. Sci. 25, 2679-2689 (1990).

18. Naidich, Y. Progress in Surface and Membrane Science. (Academic Press, 1981).

19. Smith, J. R. \& Zhang, W. Stoichiometric interfaces of Al and Ag with Al2O3. Acta Mater. 48, 4395-4403 (2000).

20. Finnis, M. W. The theory of metal-ceramic interfaces. J. Phys. Condens. Matter 8, 5811-5836 (1996).

21. Liu, W., Li, J. C., Zheng, W. T. \& Jiang, Q. NiAl (110) Cr (110) interface: A density functional theory study. Phys. Rev. B - Condens. Matter Mater. Phys. 73, 205421 (2006).

22. Mills, K. C. \& Su, Y. C. Review of Surface Tension Data for Metallic Elements and Alloys: Part 1-Pure Metals. Int. Mater. Rev. 51, 329-351 (2006).

23. Landry, K. \& Eustathopoulos, N. Dynamics of wetting in reactive metal/ceramic systems: Linear spreading. Acta Mater. 44, 3923-3932 (1996).

24. Landry, K., Rado, C., Voitovich, R. \& Eustathopoulos, N. Mechanisms of reactive wetting: The question of triple line configuration. Acta Mater. 45, 3079-3085 (1997). 
25. Iwamoto, C. \& Tanaka, S. I. Reactive wetting of Ag-Cu-Ti on SiC in HRTEM. Acta Mater. 46, 2381-2386 (1998).

26. Pham, T. et al. A Universal Wet-Chemistry Route to Metal Filling of Boron Nitride Nanotubes. Nano Lett. 16, 320-325 (2016).

27. Golberg, D., Xu, F. F. \& Bando, Y. Filling boron nitride nanotubes with metals. Appl. Phys. A Mater. Sci. Process. 76, 479-485 (2003).

28. Qian, Q. et al. Convenient synthesis of Fe-filled boron nitride nanotubes by SHS method. Mater. Lett. 65, 866-868 (2011).

29. Oku, T., Koi, N., Narita, I., Suganuma, K. \& Nishijima, M. Formation and atomic structures of boron nitride nanotubes with cup-stacked and Fe nanowire encapsulated structures. Mater. Trans. 48, 722-729 (2007).

30. Golberg, D. \& Bando, Y. Metal-filled boron nitride nanotubes. AIP Conf. Proc. 633, 145-150 (2002).

31. Tang, C., Bando, Y., Golberg, D., Ding, X. \& Qi, S. Boron nitride nanotubes filled with Ni and NiSi2 nanowires in situ. J. Phys. Chem. B 107, 6539-6543 (2003).

32. Golberg, D., Bando, Y., Mitome, M., Fushimi, K. \& Tang, C. Boron nitride nanotubes as nanocrucibles for morphology and phase transformations in encapsulated nanowires of the Mg-O system. Acta Mater. 52, 3295-3303 (2004).

33. Golberg, D., Bando, Y., Kurashima, K. \& Sato, T. Nanotubes of boron nitride filled with molybdenum clusters. J. Nanosci. Nanotechnol. 1, 49-54 (2001).

34. Man, W. Q., Chang, C. W. \& Zettl, A. Encapsulation of one-dimensional potassium halide crystals within BN nanotubes. Nano Lett. 4, 1355-1357 (2004).

35. Mickelson, W., Aloni, S., Han, W. Q., Cumings, J. \& Zettl, A. Packing C60 in boron nitride nanotubes. Science (80-. ). 300, 467-469 (2003).

36. Chen, L., Ye, H. \& Gogotsi, Y. Synthesis of boron nitride coating on carbon nanotubes. J. Am. Ceram. Soc. 87, 147-151 (2004).

37. Shen, Z. Q. et al. Boron nitride nanotubes filled with zirconium oxide nanorods. $J$. Mater. Res. 17, 2761-2764 (2002).

38. Ma, R., Bando, Y., Sato, T. \& Kurashima, K. Thin boron nitride nanotubes with unusual large diameters. Chem. Phys. Lett. 350, 434-440 (2001).

39. Han, W., Redlich, P., Ernst, F. \& Ruhle, M. Synthesizing boron nitride nanotubes filled with SiC nanowires by using carbon nanotubes as templates. Appl. Phys. Lett. 75, 1875-1877 (1999). 
40. Dujardin, E., Ebbesen, T. W., Hiura, H. \& Tanigaki, K. Capillarity and wetting of carbon nanotubes. Science (80-. ). 265, 1850-1852 (1994).

41. Golberg, D., Bando, Y., Kurashima, K. \& Sato, T. Synthesis and characterization of ropes made of BN multiwalled nanotubes. Scr. Mater. 44, 1561-1565 (2001).

42. Laha, T., Agarwal, A., McKechnie, T. \& Seal, S. Synthesis and characterization of plasma spray formed carbon nanotube reinforced aluminum composite. Mater. Sci. Eng. A 381, 249 (2004).

43. Xu, J. Q., Chen, L. Y., Choi, H. \& Li, X. C. Theoretical study and pathways for nanoparticle capture during solidification of metal melt. J. Phys. Condens. Matter 24, 255304 (2012).

44. Bakshi, S. R., Singh, V., Seal, S. \& Agarwal, A. Aluminum composite reinforced with multiwalled carbon nanotubes from plasma spraying of spray dried powders. Surf. Coatings Technol. 203, 1544-1554 (2009).

45. Sampath, S. \& Herman, H. Rapid solidification and microstructure development during plasma spray deposition. J. Therm. Spray Technol. 5, 445 (1996).

46. Voytovych, R., Bougiouri, V., Calderon, N. R., Narciso, J. \& Eustathopoulos, N. Reactive infiltration of porous graphite by NiSi alloys. Acta Mater. 56, 2237-2246 (2008).

47. Camel, D., Drevet, B. \& Eustathopoulos, N. Capillarity in the processing of photovoltaic silicon. J. Mater. Sci. 51, 1722-1737 (2016). 


\section{CHAPTER 7}

\section{APPLICATION OF BORON NITRIDE NANOTUBE FOR REINFORCING MAGNESIUM ALLOY}

Magnesium alloys, the lightest structural alloys (with a density of $1.74 \mathrm{~g} \mathrm{~cm}^{-3}$ ), are increasingly gaining popularity for aerospace and automotive applications. However, the stiffness, hardness, strength, and creep-resistance of commercial $\mathrm{Mg}$ alloys do not measure up for critical load-bearing applications where steel, titanium or high-strength aluminum alloys are used ${ }^{1,2}$. The addition of BNNT to $\mathrm{Al}$ and $\mathrm{Ti}$ matrices has been reported to improve their tensile strength ${ }^{3-5}$, compressive strength $^{6}$, elastic modulus ${ }^{7}$, hardness ${ }^{4-8}$, and high-temperature strength ${ }^{9}$. However, the integration of BNNT in Mg matrices has not been explored yet. This chapter presents the processing, microstructure evolution and strengthening mechanisms due to the addition of BNNT to AZ31 magnesium alloy (Mg-3 wt.\% Al-1 wt.\% Zn).

\subsection{Sandwich Architecture to Suppress Embrittlement}

A major challenge with $\mathrm{Mg}$ alloys is their low ductility ${ }^{10}$ since they have only one closepacked plane (basal plane) and slip is primarily confined to this plane at room temperature. The problem of limited ductility is further exacerbated by adding secondary fillers, which enhance strength and stiffness at the cost of ductility. This necessitates a careful composite design and processing to enhance the load-bearing capability of $\mathrm{Mg}$ alloys without compromising their plasticity. If the nanofillers are distributed throughout the metal matrix, they obstruct dislocation motion and can cause a build-up of dislocation forests, thereby 
hampering the plasticity of the metal. A sandwich design approach, where the composite consists of matrix/filler multi-layer architecture (Figure 7.1), can minimize the obstruction of dislocation motion. While the dislocation activity won't be severely restricted in the metallic layers, the intermediate reinforcement layer is expected to provide superior resistance to failure. High pressure spark plasma sintering technique was employed to ensure good bonding between AZ31 and BNNTs.

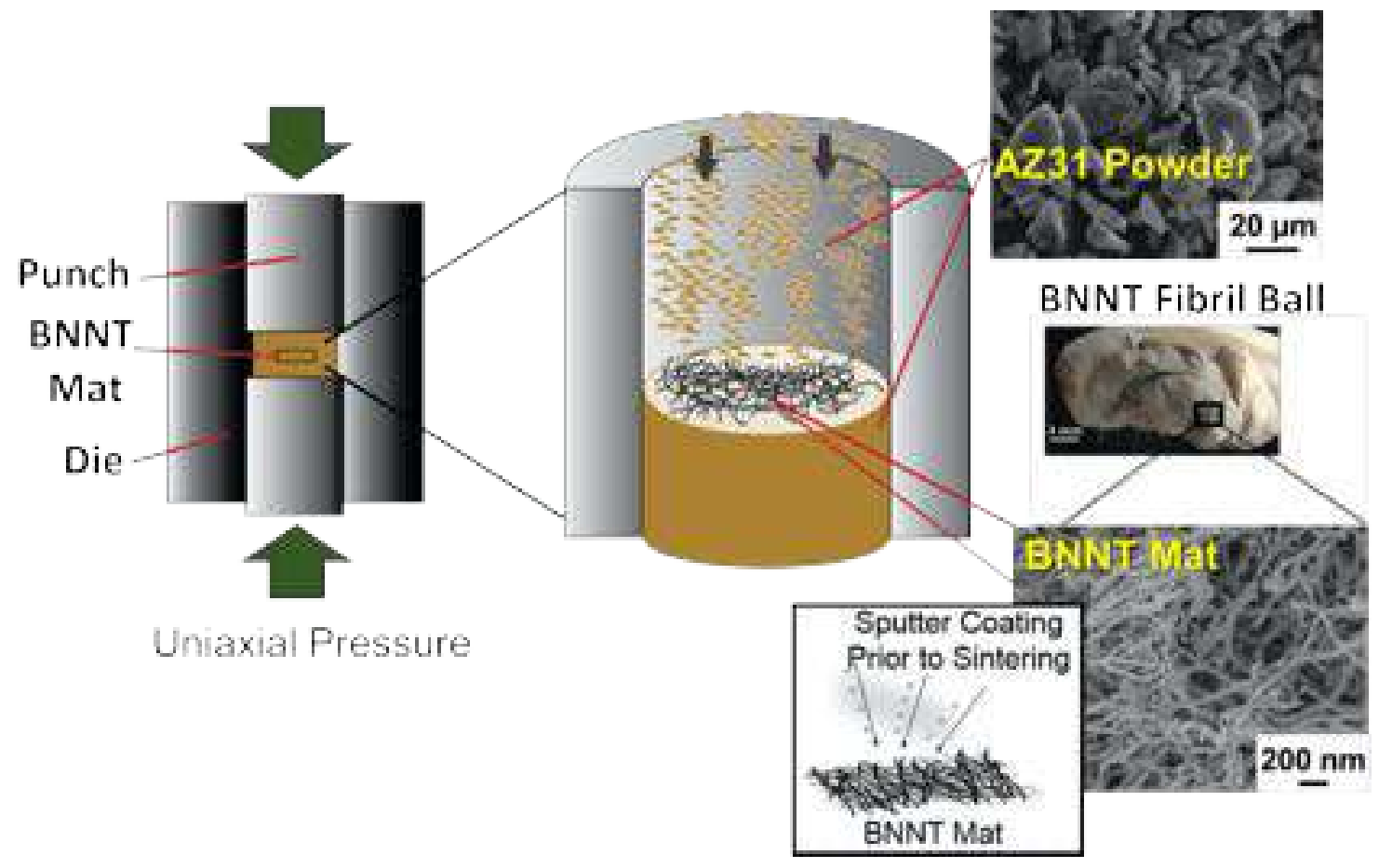

Figure 7.1. Processing scheme of AZ31-BNNT sandwich-structured composite by highpressure spark plasma sintering.

\subsection{Integration of BNNT in AZ31}

$\mathrm{X}$-ray diffraction characterization confirms the retention of the $\mathrm{h}-\mathrm{BN}$ phase after sintering (Figure 7.2a), indicating the nanotubes do not undergo significant phase transformation during processing. There was a minor peak corresponding to magnesium oxide, which is 

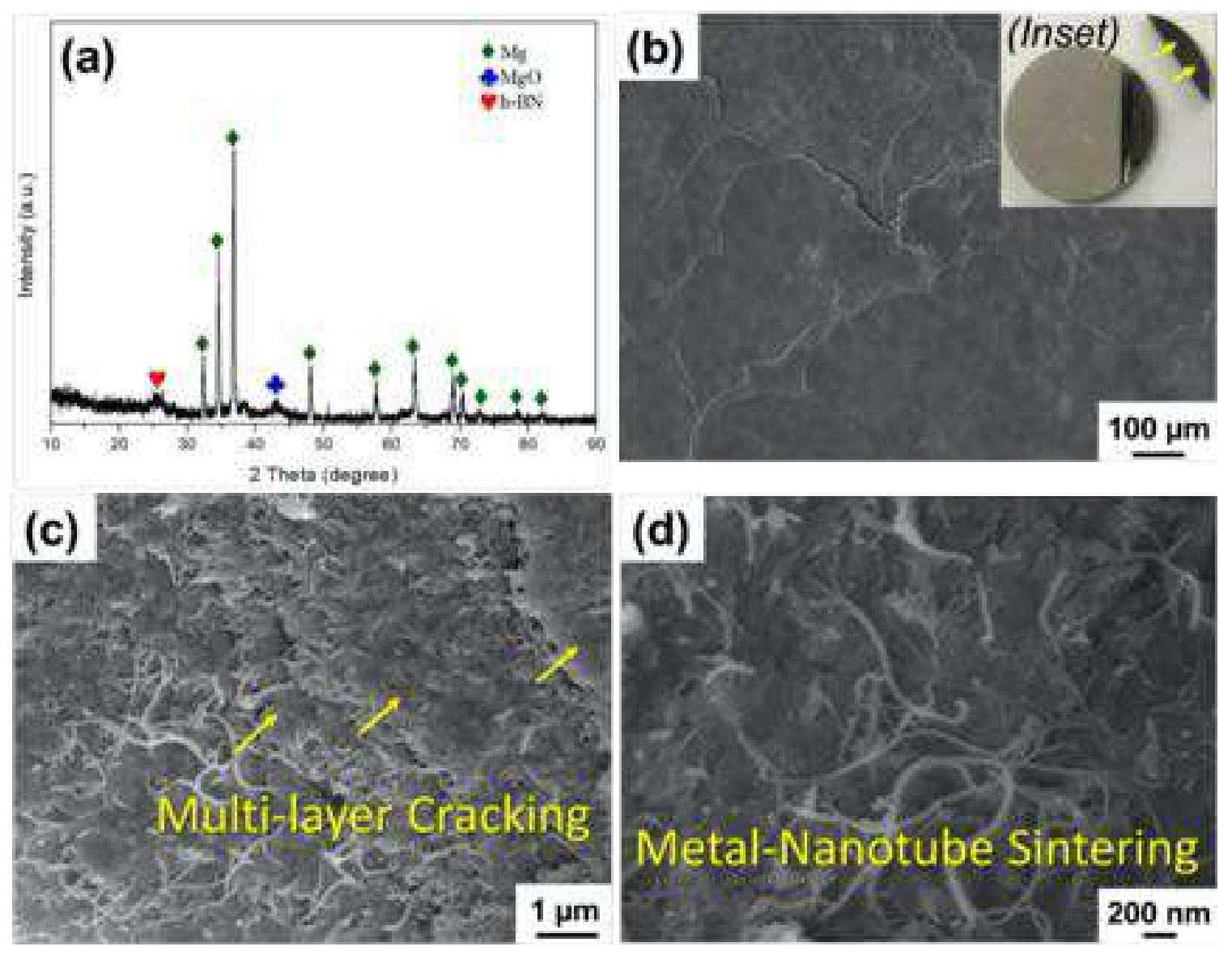

Figure 7.2. (a) X-ray diffraction plot showing h-BN, $\mathrm{Mg}$, and $\mathrm{MgO}$ in the sintered pellet, (b) low magnification SEM micrograph revealing multiple crack deflection events (inset: image of the sectioned and delaminated pellet used for imaging), (c) SEM micrograph showing a stepped surface and multi-layer cracking due to sectioning, and (d) high magnification SEM micrograph demonstrating impregnation and intimate bonding of alloy with the nanotubes.

expected due to the native oxide of the starting powder particles. In order to microscopically evaluate the microstructure and the integration of BNNT in the composite, the sintered pellet was sectioned and delaminated along the sandwiched layer. The sectioning was performed using a slow-speed diamond blade to limit undesirable plastic deformation. The inset in Figure 7.2b shows the sectioned sample. The exposed interface was then observed under a field emission scanning electron microscope. The low 
magnification image in Figure 7.2b shows multiple crack deflection events. The delamination was not smooth and a single plane event - instead, there seems to be a multilayer detachment. The higher magnification micrograph shown in Figure 7.2c reveals that a network of nanotubes along the interface prevents continuous cracking under cutting forces. This leads to extensive crack deflection, manifesting as a stepped surface post delamination. Strong bonding between BNNT and the metal matrix is essential for crack deflection to take place. This is evidenced from the micrograph shown in Figure 7.2d, where the signature of fusion between the alloy powder and the nanotubes are seen. Under the applied pressure during SPS, the powder particles can impregnate into the porous BNNT mat layer. The impregnated particles are then wrapped by the nanotubes during sintering, resulting in a high net contact area for interface bonding. Plastic deformation of the powder particles under the punch pressure aids in intimate alloy/BNNT attachment and the formation of dense nanocomposite clusters at the interface (as shown in Figure 7.2d).

\subsection{Metal-Nanotube Interface Analysis}

In addition to the mechanical forces, elevated temperature exposure during SPS promotes thermal diffusion. High-resolution transmission electron microscopy (HRTEM) investigations were performed to interrogate the metal-nanotube interactions. A TEM sample was prepared from the sandwiched interface region by focused ion beam machining. Figure 7.3a shows a scanning-TEM (STEM) micrograph of the interface region. An elemental line profile (by energy-dispersive X-ray spectroscopy) demonstrates the transition from a predominantly BNNT-rich region (near Pt. 1) to the Mg alloy layer 
(around Pt. 2). The elemental information was then utilized to zoom in and image the interfaces in the BNNT-rich regions. HRTEM imaging shows a dense network of BNNT with a sintered alloy occupying the inter-tube gaps (Figure 7.3b). This is indicative of prominent plastic flow or localized extrusion of AZ31 during SPS (400 MPa pressure), enabling effective interface consolidation.

Lattice spacing calculations by fast Fourier transform (FFT) analysis confirmed the h-BN phase of the nanotubes (Figure 7.4a), which establishes that the nanotubes are stable and withstand the processing conditions chosen in this work. HRTEM also revealed a minimal interfacial reaction between the nanotubes and the matrix. Figures $\mathbf{7 . 4 b}$, c demonstrate the presence of magnesium nitride and aluminum nitride in the compositeclusters, indicating there were some reactions during sintering. Thermodynamic calculations were performed using the Equilib module of FactSage thermochemical software $^{11}$ to gain insights into the interfacial reactions. The SPS processing temperature and pressure conditions were utilized for the analysis. First, the sandwiched BNNT is expected to react with the thin $(1 \mu \mathrm{m})$ sputtered coatings of pure $\mathrm{Mg}$ (deposited on both sides of the mat). The thermochemical analysis predicted the following reaction based on Gibbs energy minimization:

$$
\begin{aligned}
& 0.0124 g B N+0.000434 g M g=0.0122 g B N+0.00045 g g_{3} N_{2}+ \\
& 0.00021 g M g B_{2}, \Delta \mathrm{G}=-0.123 \mathrm{~J}
\end{aligned}
$$

Subsequently, BNNT interacts with the AZ31 alloy powder in the die, and the following reaction is thermodynamically expected: 
(a)
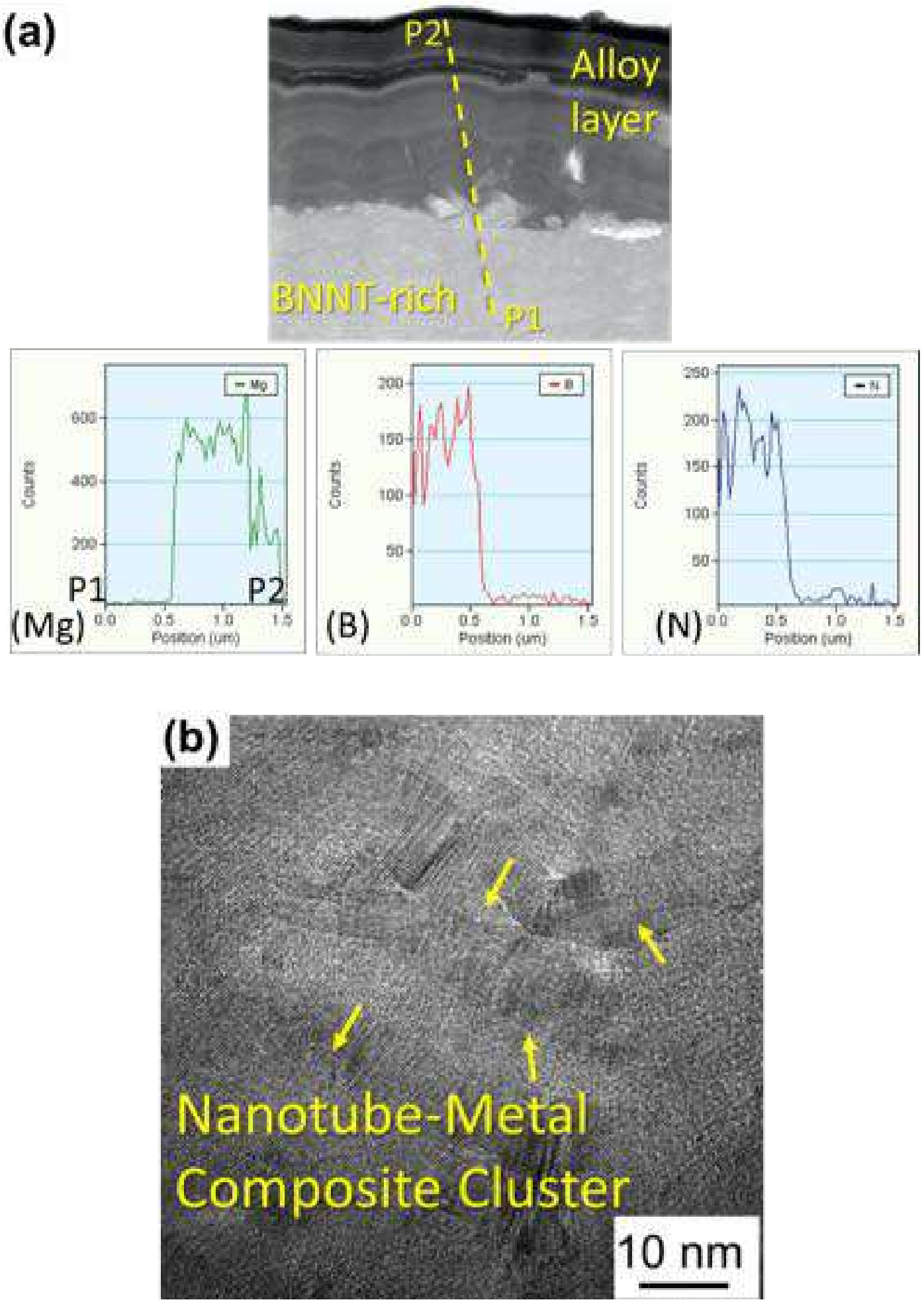

Figure 7.3. (a) STEM imaging and the corresponding elemental line profile along the alloy-BNNT interface region, and (b) HRTEM image showing a dense network of BNNTs in the composite. 

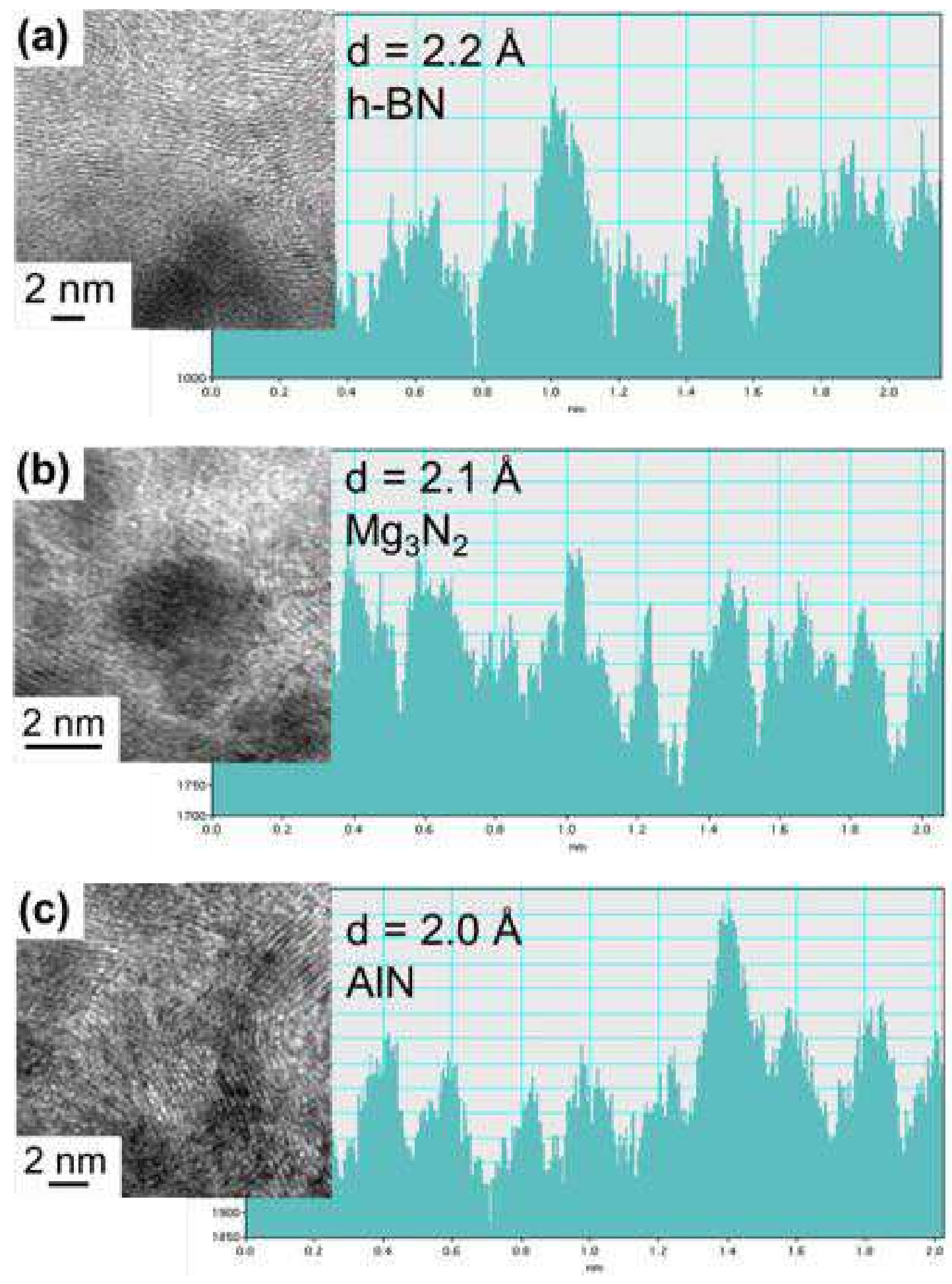

Figure 7.4. Lattice spacing calculations confirming: (a) h-BN phase of the nanotubes, (b) $\mathrm{Mg}_{3} \mathrm{~N}_{2}$ formation, and (c) AIN formation due to AZ31-BNNT reaction. 


$$
\begin{aligned}
& \quad 0.0122 g B N+1.181 g M g+0.0369 \mathrm{Al}+0.0123 g \mathrm{Zn}=1.159 \mathrm{gMg}+ \\
& 0.0391 g \mathrm{Al}_{29} \mathrm{Mg}_{21}+0.0201 \mathrm{gAlN}+0.0123 \mathrm{~g} \mathrm{Zn}+0.0113 g \mathrm{MgB} B_{2} \\
& \Delta \mathrm{G}=-53.473 \mathrm{~J}
\end{aligned}
$$

Negative $\Delta \mathrm{G}$ confirms the thermodynamic feasibility of the formation of $\mathrm{Mg}_{3} \mathrm{~N}_{2}$ and AlN products observed via TEM (Figures 7.4b, 3c). These reaction products were not homogeneously observed throughout the nanotube interface, rather they were scarce and scattered. The reaction kinetics can become a rate-determining step during SPS due to short processing times. This explains why the h-BN phase is retained in the composite (Figures 7.2a, 7.3b and 7.3c) even though the thermochemical calculations predict the complete consumption of $\mathrm{BN}$ (Reaction 7.2). Kinetics constraints are likely responsible for the absence/lack of detection of $\mathrm{MgB}_{2}$ and $\mathrm{Al}_{29} \mathrm{Mg}_{21}$ phases predicted by FactSage. Controlled reactions are desirable for strong metal/nanotube bonding due to the nano-interphase(s) which act as anchors between the matrix and the filler. The covalently bonded interfaces are intimate, firm, and ideal for matrix-to-filler stress-transfer during mechanical loading ${ }^{12}$. It is noteworthy, though, that excess interfacial reaction is undesirable as that can significantly degrade the morphology and mechanical properties of the nanotubes. Rapid consolidation during SPS is ideal for minimizing high-temperature exposure, and hence the chemical reactions are minimal.

\subsection{Strengthening Mechanisms}

An in-situ double cantilever experimental setup was used to examine the mechanisms associated with BNNT reinforcement in the sandwich composite. The test was performed 
inside the SEM chamber for high-resolution imaging of failure characteristics. Mechanical loading resulted in crack propagation and de-bonding along the composite interface. BNNTs resisted crack opening, evidenced by the stretching and fracture of the nanotubes (Figure 7.5a). The AZ31-BNNT composite clusters act as bridges or anchors at the interface, which is shown in Supplementary Video V7.1. The crack bridges were observed to undergo re-alignment - from being parallel to the interface before mechanical loading to becoming angled bridges - as the loading progress. The realignment is advantageous since the nanotubes display load-bearing ability along the axial direction. Further forces were expended on the snapping of these bridges before crack propagation (Figure 7.5b). The fracture strength of BNNT is remarkably high ( $30 \mathrm{GPa})$, which is effectively exploited in this sandwich composite to provide failure resistance. This is demonstrated in Supplementary Video V7.2 showing the breaking of metal-nanotube clusters under continued mechanical loading. The bridge failure was ductile, evidenced by the slow nanotube pull-out seen in the video.

After the conclusion of the test, the delaminated surfaces were imaged and extensive crack bridging was observed due to the high-aspect-ratio of the nanotubes (Figure 7.5c). Figure 7.5d shows the pull-out of nanotubes in the delaminated surface, confirming effective stress transfer from the (weaker) metal matrix to the (stronger) nanotubes. We are not presenting any quantitative stress-strain data here, which can be a subject of future investigations. The objective of the in-situ tests performed here is to qualitatively evaluate the strengthening mechanisms and efficacy of BNNTs in real-time. 

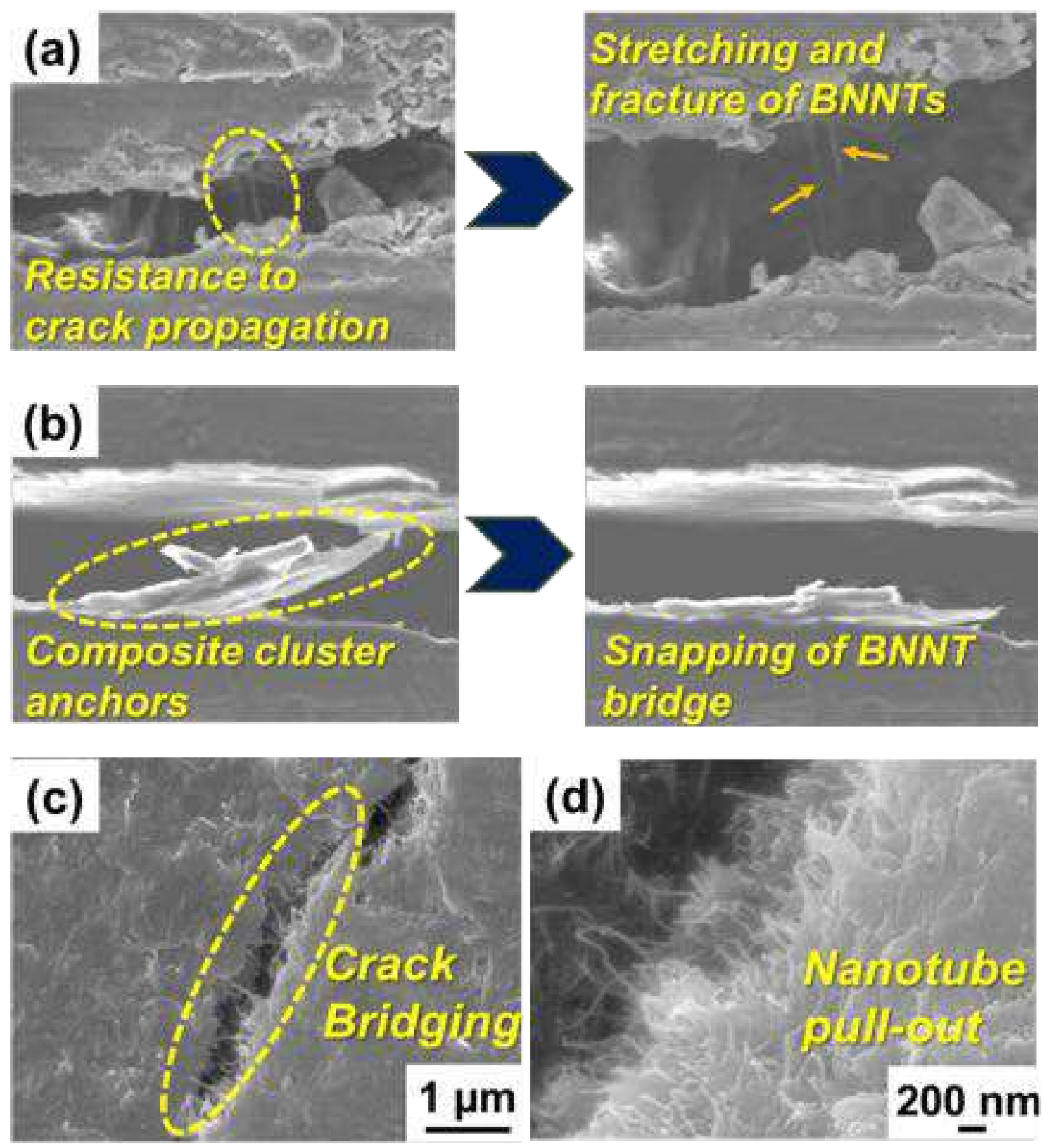

Figure 7.5. In-situ double cantilever testing of AZ31-BNNT composite with real-time SEM imaging: (a) stretching and fracture of BNNTs resisting crack opening, and (b) snapping of composite cluster acting as interface anchor. Post-delamination imaging showing (c) extensive crack bridging and (d) nanotube pull-out due to mechanical loading. 


\subsection{Summary}

SPS is an effective technique to engineer BNNT-reinforced $\mathrm{Mg}$ alloy sandwich composites. A combination of high pressures and elevated temperatures during processing allows rapid sintering and bonding of AZ31 and BNNT. Limited interfacial reactions are helpful for effective stress-transfer between the matrix and the nanotubes. The in-situ mechanical investigation highlights crack-bridging due to BNNT in a sandwich-structured composite, providing superior failure-resistance. These findings are promising, and we envision BNNT to be a highly effective reinforcement candidate for augmenting the mechanical properties of lightweight magnesium.

\section{Output: Published Article}

P. Nautiyal, N. Denis, T. Dolmetsch, C. Zhang, B. Boesl, A. Agarwal, Interface Engineering and Direct Observation of Strengthening Behavior in Field-Sintered Boron Nitride Nanotube-Magnesium Alloy Composite, Advanced Engineering Materials, DOI: 10.1002/adem.202000170 (2020).

\section{References}

1. Habibi, M. K., Joshi, S. P. \& Gupta, M. Hierarchical magnesium nano-composites for enhanced mechanical response. Acta Mater. 58, 6104-6114 (2010).

2. Zhong, X. L., Wong, W. L. E. \& Gupta, M. Enhancing strength and ductility of magnesium by integrating it with aluminum nanoparticles. Acta Mater. 55, 63386344 (2007).

3. Yamaguchi, M. et al. Utilization of multiwalled boron nitride nanotubes for the reinforcement of lightweight aluminum ribbons. Nanoscale Res. Lett. 8, 3 (2013).

4. Xue, Y. et al. Aluminum matrix composites reinforced with multi-walled boron nitride nanotubes fabricated by a high-pressure torsion technique. Mater. Des. 88, 451-460 (2015). 
5. Yamaguchi, M., Meng, F., Firestein, K., Tsuchiya, K. \& Golberg, D. Powder metallurgy routes toward aluminum boron nitride nanotube composites, their morphologies, structures and mechanical properties. Mater. Sci. Eng. A 604, 9-17 (2014).

6. Lahiri, D. et al. Boron nitride nanotubes reinforced aluminum composites prepared by spark plasma sintering : Microstructure, mechanical properties and deformation behavior. Mater. Sci. Eng. A 574, 149-156 (2013).

7. Antillon, M., Nautiyal, P., Loganathan, A., Boesl, B. \& Agarwal, A. Strengthening in Boron Nitride Nanotube Reinforced Aluminum Composites Prepared by Roll Bonding. Adv. Eng. Mater. 20, 1800122 (2018).

8. Singhal, S. K., Srivastava, A. K., Pasricha, R. \& Mathur, R. B. Fabrication of AlMatrix Composites Reinforced with Amino Functionalized Boron Nitride Nanotubes. J. Nanosci. Nanotechnol. 11, 5179-5186 (2011).

9. Bhuiyan, M. M. H. et al. Boron nitride nanotube reinforced titanium metal matrix composites with excellent high-temperature performance. J. Mater. Res. 32, 3744 3752 (2017).

10. Trang, T. T. T. et al. Designing a magnesium alloy with high strength and high formability. Nat. Commun. 9, 2522 (2018).

11. Bale, C. W. et al. Reprint of: FactSage thermochemical software and databases, 2010-2016. Calphad Comput. Coupling Phase Diagrams Thermochem. 55, 1-19 (2016).

12. Bakshi, S. R. \& Agarwal, A. An analysis of the factors affecting strengthening in carbon nanotube reinforced aluminum composites. Carbon N. Y. 49, 533-544 (2011). 


\section{CHAPTER 8}

\section{IN-SITU INVESTIGATION OF DEFORMATION MECHANISMS INDUCED BY BORON NITRIDE NANOTUBES AND NANO- INTERPHASES IN Ti-6Al-4V}

Unraveling the interface mechanics holds key to engineer MMCs with the predictable and desired mechanical response. The formation of additional interfacial products during processing can lead to multiple deformation mechanisms in the composite. These transformations are exacerbated in Titanium alloys because of higher processing temperatures $\left(>700^{\circ} \mathrm{C}\right)$, unlike $\mathrm{Al}$ and $\mathrm{Mg}$ alloys, which require modest sintering

temperatures $\left(<600^{\circ} \mathrm{C}\right)^{1-4}$. Therefore, this chapter of the dissertation delves into detailed high-resolution in-situ investigation of deformation mechanisms in Ti6Al4V-BNNT composites. Two composite specimens are investigated, one sintered at a relatively lower temperature $\left(750^{\circ} \mathrm{C}\right)$, and the other at a higher temperature $\left(950^{\circ} \mathrm{C}\right)$. These composites are henceforth referred to as LT and HT composites, respectively. The central aspiration of this work is to establish processing-interface chemistry-mechanics correlation in these highly hierarchical nanocomposites.

\subsection{Background: Sintering of BNNT-reinforced Ti6Al4VAlloy}

Sintering of Ti6Al4V alloy has been reported using a wide range of consolidation temperatures, varying from $\sim 800^{\circ} \mathrm{C}$ to $\sim 1400^{\circ} \mathrm{C}$. The upper end of the temperature spectrum is not suitable for fabricating BNNT-reinforced composites due to the enhanced reactivity of the nanotubes. Prior studies on high-temperature exposure of BNNT have 
revealed oxidative transformations seem to initiate in a temperature range of $750-1000^{\circ} \mathrm{C}^{5-}$ ${ }^{7}$. These thermal degradations can be arrested by vacuum or inert environment sintering. Nevertheless, the controlled environment will only limit oxidation. The interfacial reactions between BNNT and alloy are expected to be accentuated at higher sintering temperatures. In a recent study, Bustillos reported the formation of TiB and TiN phases during spark plasma sintering of Ti6Al4V-BNNT composite ${ }^{8}$. In order to control the reactions, the study explored low-temperature sintering of the composite $\left(650\right.$ and $\left.750^{\circ} \mathrm{C}\right)$. The reduction in temperature was compensated by raising the sintering pressure to achieve acceptable densification and bonding ${ }^{9}$. Additionally, short sintering time (10 minutes) was chosen to impose a kinetic barrier to chemical reactions. The work demonstrated arrested interfacial reaction products when low-temperature conditions were used. The findings are promising as they signal the ability to tune and tweak the interface chemistry in BNNTreinforced composites. It is well understood that interface chemistry greatly influences the mechanics of $\mathrm{MMCs}^{10,11}$. Bustillo's work attempted to establish this correlation by examining the nanoindentation and compression response of the composites sintered under different conditions ${ }^{8}$. The study showed remarkable variation in properties despite adding the same amount of BNNT filler, attributed to interfacial interactions and reaction products. There is an opportunity to tailor stress-transfer in the composite, but this also poses questions regarding the transitions in deformation characteristics in the composite as a function of the interface structure. 


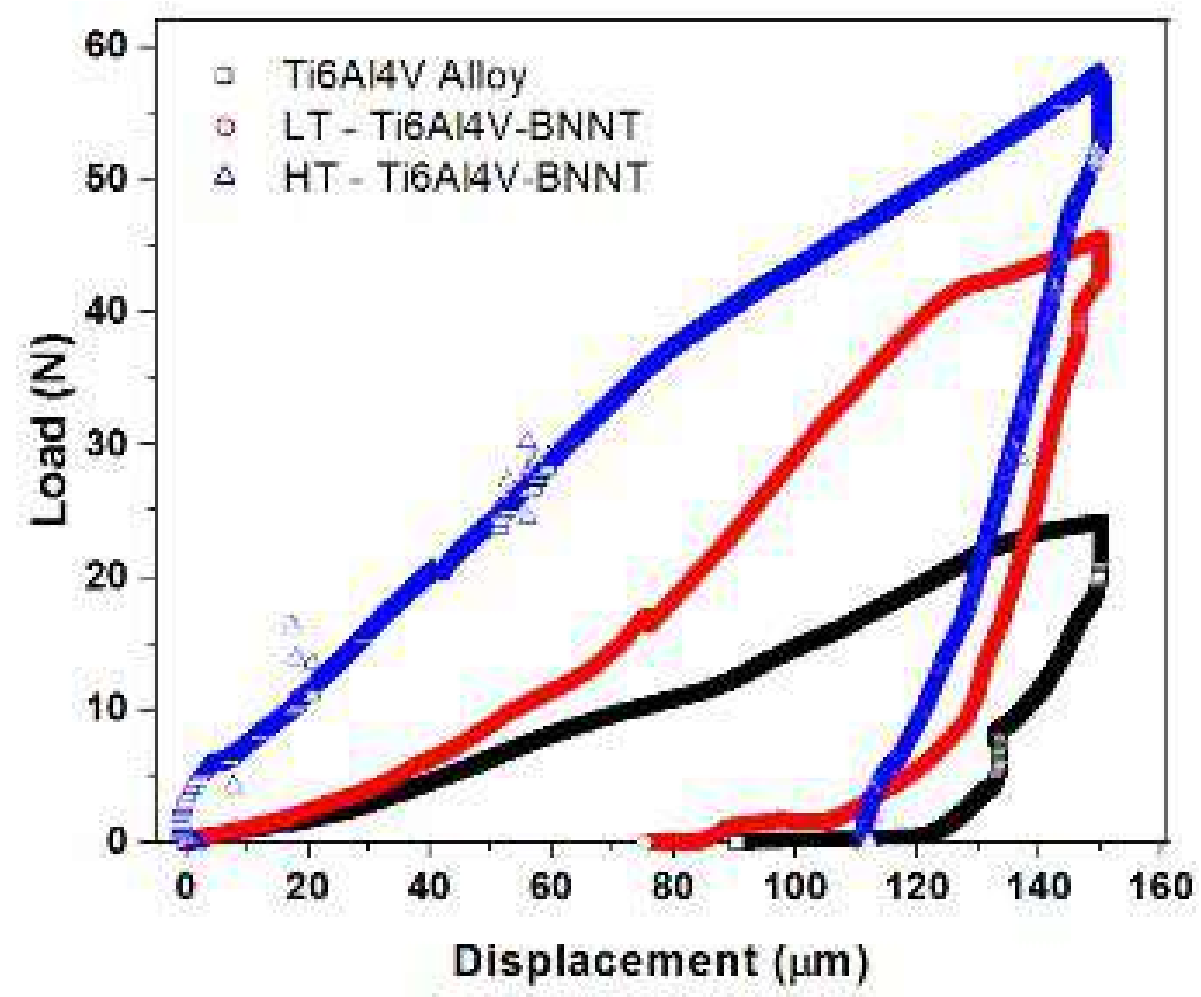

Figure 8.1. Comparison of meso-scale indentation curves for Ti6Al4V alloy and BNNTreinforced Ti6Al4V composites (LT and HT-sintering conditions).

\subsection{BNNT-induced Strengthening: Ex-situ Mechanical Investigations}

Overall mechanical properties of composites are determined by the interplay of mechanisms between multiple species in the microstructure, such as metal particles, nanotubes, and reaction products. The bonding of metal particles and the interface interactions between filler and matrix are as important as the intrinsic properties of the individual constituents. Therefore, we investigated meso-scale deformation of the composites by high load indentation (with peak forces in the order of $10^{1}$ to $10^{2} \mathrm{~N}$ ). Mesoscale testing captures the response of material volume intermediate between bulk-scale and highly localized nano-/micro-indentation. As a result, these tests capture the mechanical 
interactions between the constituents in the composite microstructure. Multiple indents were made in different regions of the samples, with penetration depths varying from 150 $\mu \mathrm{m}$ to $1,000 \mu \mathrm{m}$. Figure $\mathbf{8 . 1}$ shows the comparison of representative load-displacement curves comparing the composites against the pure alloy, all captured in displacement control mode at $150 \mu \mathrm{m}$ indentation depth. The plot confirms the reinforcement effect of BNNT added to Ti6Al4V alloy, as the penetration forces required to achieve the same indentation depth of $150 \mu \mathrm{m}$ are higher for composite samples. The LT composite requires an indentation force of $\sim 45.5 \mathrm{~N}$, which is approximately two times the forces required for the pristine alloy. Further enhancement in peak forces was recorded for HT composite ( $\sim 7$ $\mathrm{N}$ for $150 \mu \mathrm{m}$ displacement). The findings demonstrate superior load-bearing ability in HT composite.

In order to study the deformation mechanisms activated in the composites, the residual deformation regions were imaged after the indentation tests under SEM (Figure 8.2). The SEM images correspond to indents made at varying depths ( 150 to $1,000 \mu \mathrm{m})$ to decipher multi-scale mechanisms. Plastic deformation was the predominant mechanism exhibited by LT composite at relatively lower displacements $(<500 \mu \mathrm{m})$, shown in Figure 8.2a. Some of the indents were characterized by a nominal pile-up of material. Additionally, limited cracking was observed in the vicinity of the indent at higher indentation depths of $750-1,000 \mu \mathrm{m}$. The cracks were seen to propagate along the prior particle boundaries due to the coalescence of inter-particle pores seen in the micrographs. High magnification imaging of cracks revealed extensive bridging action due to high aspect ratio nanotubes. Bridge formation signifies the resistance to crack propagation and crack opening during indentation loading. Effective bridging (instead of pull-out or sliding) 
indicates strong bonding between BNNT and alloy. This finding supports the feasibility of low temperature sintering to integrate $\mathrm{BNNT}$ in the $\mathrm{Ti}$ matrix with strong interface adhesion.

\section{(a) Ti6Al4V-BNNT (LT)}
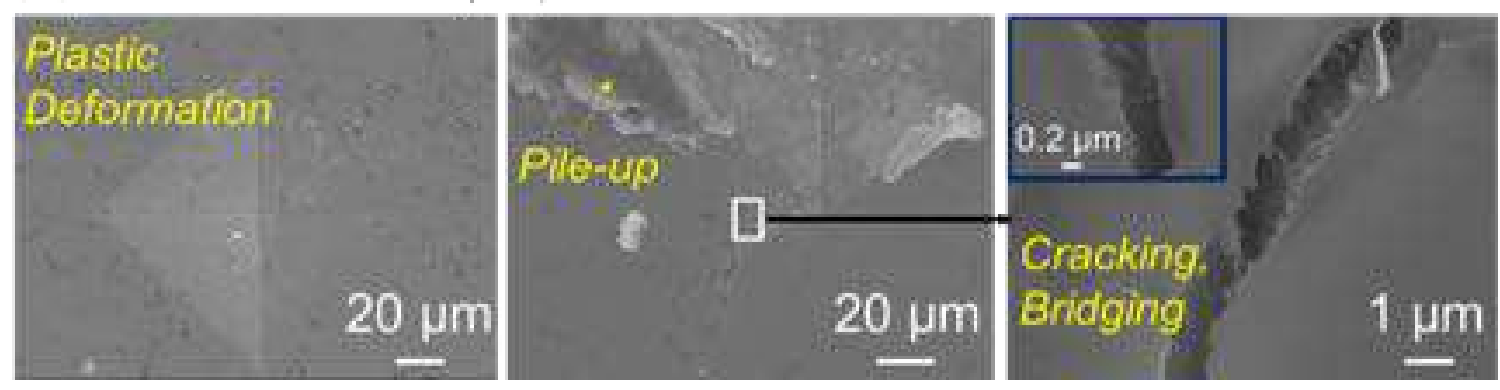

(b) Ti6Al4V-BNNT (HT)
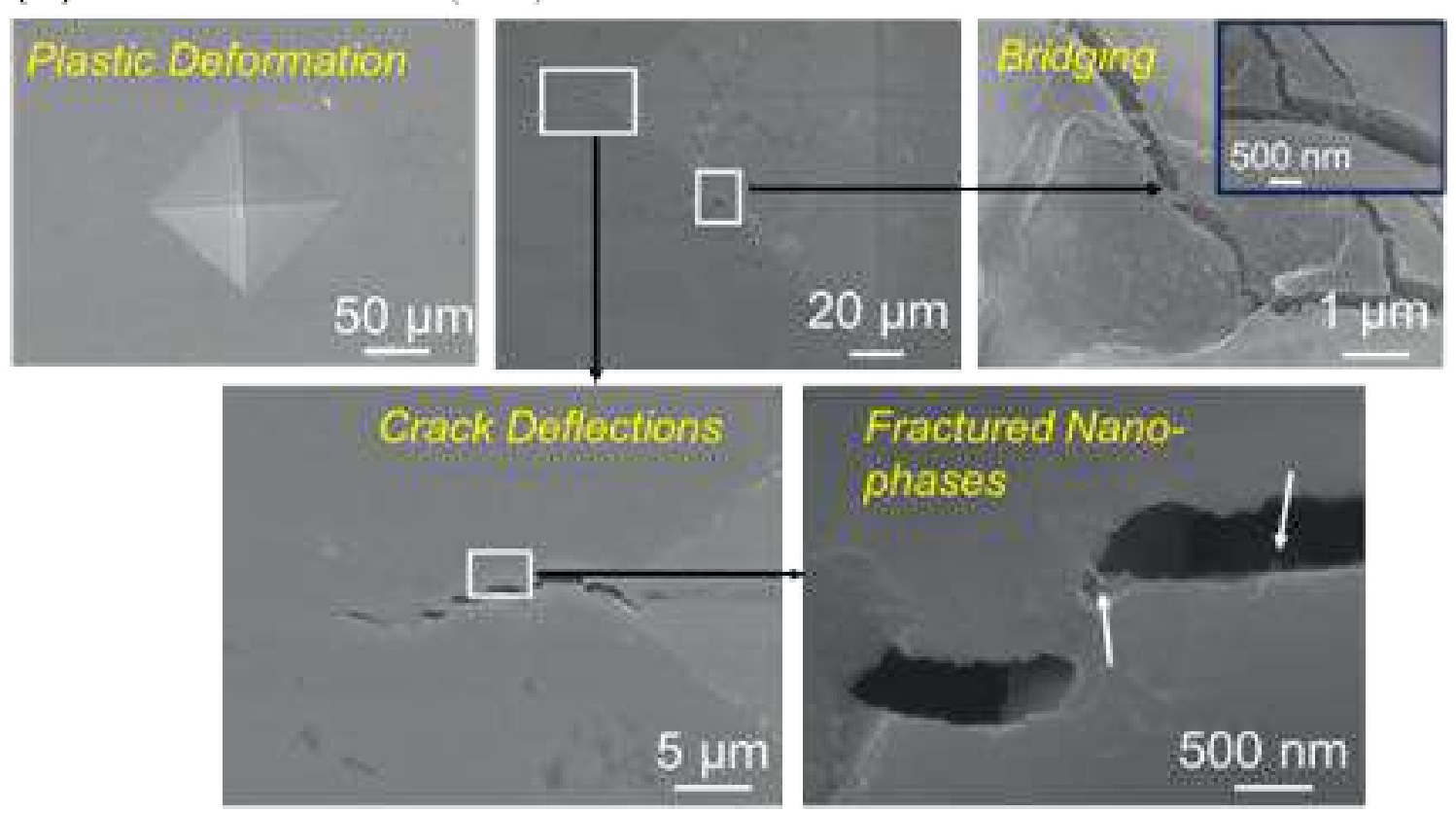

Figure 8.2. Post indentation FESEM imaging of deformation in (a) LT-sintered, and (b) HT-sintered Ti6Al4V-BNNT composites.

HT composite exhibited a combination of plastic deformation and extensive cracking under indentation loading (Figure 8.2b). Cracks were seen within the indentation 
zone as well as emanating from the indent edges (propagating away from the deformation region). Unlike LT composite, where cracks were seen along the porous particle interfaces; HT composite seems to display trans-granular cracking. BNNT bridges were observed by high magnification SEM imaging. Another notable observation is extensive crack deflection and needle-like fractured species protruding from the cracked regions. It has been reported that titanium boride (TiB) has needle-like morphology ${ }^{12}$. Therefore, it is likely that interphases in the microstructure resist crack propagation. The additional reinforcement effect of interfacial products explains the superior strength of the HT composite recorded in Figure 8.1. While post-mortem imaging is useful to examine deformation characteristics displayed by composites, they do not answer several critical questions, such as:

- What are the stresses required to activate specific mechanisms in the composite?

- What is the microstructure sensitivity of the mechanisms? Are these mechanisms activated at specific length scales, and are these location-specific due to microstructure heterogeneities?

- What is the reinforcement efficacy of each of these mechanisms? How much mechanical energy is absorbed due to the activation of the mechanisms?

Therefore, in-situ investigations were carried out to obtain deeper insights into the mechanics of the Ti-BNNT composites. 
(a) Ti6Al4V Alloy

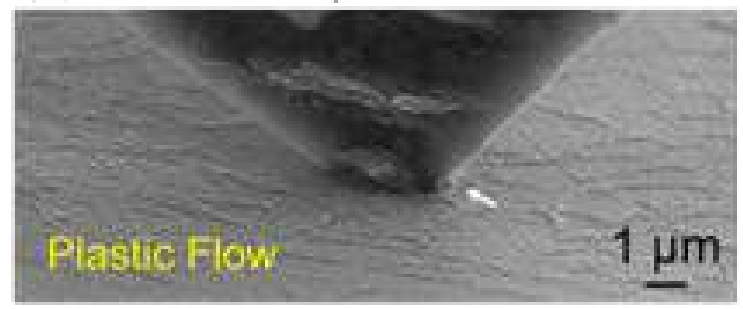

(c) Alloy-BNNT (HT) Bimodal

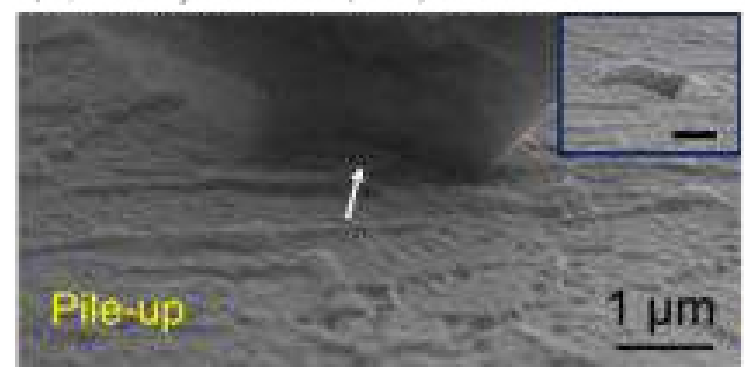

(b) Alloy-BNNT (LT)
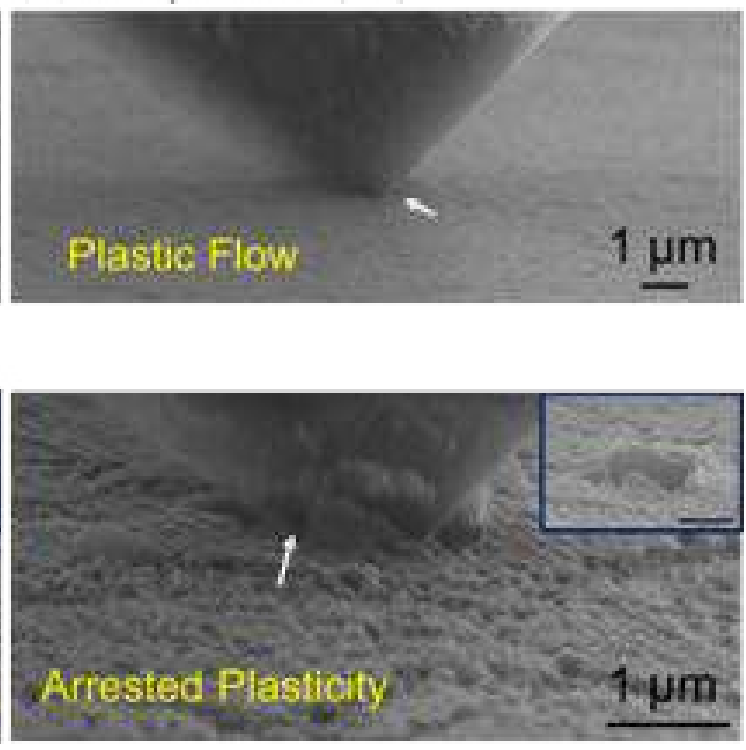

Figure 8.3. In-situ indentation for observing deformation mechanisms in Ti6Al4V alloy (a), LT Alloy-BNNT composite (b), and HT Alloy-BNNT composite (c).

\subsection{In-situ Indentation Investigation}

In-situ indentation investigations were carried out to examine the deformation mechanisms in real-time by SEM imaging. The tests were performed on polished cross-section surfaces. Indents were $1 \mu \mathrm{m}$ deep. Indentation loading of Ti6A14V alloy caused plastic flow in the vicinity of the tip, as shown in Figure 8.3a. The plastic flow was the dominant mechanism in LT composite too (Figure 8.3b and V8.1). However, the resistance to deformation was higher in the composite, evidenced by the measured indentation hardness of $10 \pm 2.1 \mathrm{GPa}$, as opposed to $7.8 \pm 0.4 \mathrm{GPa}$ recorded for the pristine alloy. BNNT addition also caused stiffening, with a jump in indentation modulus from $93.4 \pm 1.7 \mathrm{GPa}$ (alloy) to $96.6 \pm 6.7$ GPa (LT composite). In striking contrast, the mechanical response of the HT composite was rather complex. While some of the regions displayed plastic deformation and pile-up 
of material; there were other regions where the plastic flow was arrested and micro-cracks observed during tip penetration (Figure 8.3c, V8.2, and V8.3). The insets in Figure 8.3c show post-indentation SEM micrographs, emphasizing the microstructure sensitivity of the mechanisms. This manifests as bimodal distribution in mechanical measurements. Plasticity-dominated regions were characterized by hardness and indentation modulus of $11.8 \pm 2 \mathrm{GPa}$ and $95.3 \pm 2.5 \mathrm{GPa}$, respectively. These values are comparable to the measurements captured from LT composite. However, the regions with arrested plasticity displayed distinctly superior hardening $(15.5 \pm 1.9 \mathrm{GPa})$ and stiffening $(104 \pm 2.6 \mathrm{GPa})$. Non-plastic deformation mechanisms are indicative of the active role played by ceramic interphases present in HT composite.

\subsection{Unraveling Sub-Surface Deformation Mechanisms in Real Time}

It is noteworthy that in-situ indentation reveals surface-level mechanisms and much of the activity in the bulk (interior) of the sample remains unknown. These findings scratch the surface of the problem, revealing the microstructure-sensitivity of the mechanical response. It is vital to understand the internal mechanisms activated at multiple microstructural length scales in the composites for a holistic understanding. Therefore, we milled trenches in the composite samples to expose the internal structure (Figure 8.4a). We then performed indentation tests at the edge of the trench, so that the sub-surface deformation characteristics can be observed in real-time (Figure 8.4b). Multiple trenches were machined in different regions of the sample to capture distinct mechanisms arising due to microstructure heterogeneity in the composites. 

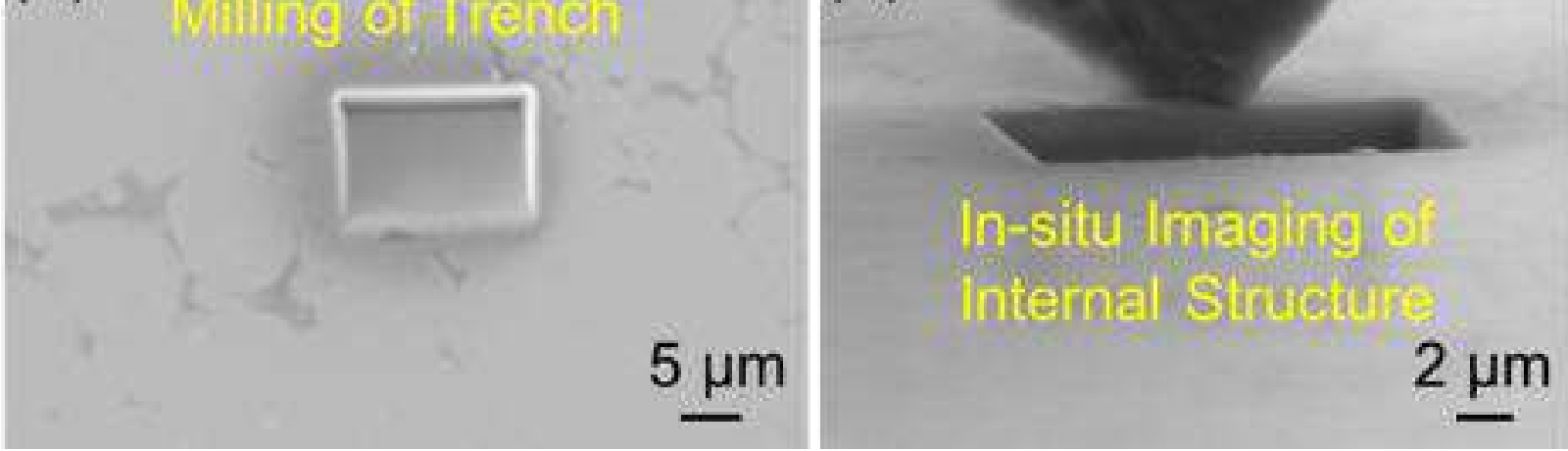

Figure 8.4. FIB milling of Ti6Al4V-BNNT composites for real time imaging of internal structure during indentation loading.

In order to capture and differentiate mechanisms arising at different load and length scales, cyclic indentation tests with incremental penetration depth were programmed. An example of the loading style is shown in Figure 8.5a. The testing was conducted in displacement control mode, and the peak indentation displacement progressively increased for each successive cycle. A total of 10 cycles were programmed, such that the first cycle involved tip penetration up to $500-750 \mathrm{~nm}$. The final cycle was usually programmed for a depth of 2,500-3,500 $\mathrm{nm}$. The loading-unloading times were relatively longer (15-30 seconds) to clearly observe the transitions in sub-surface deformation characteristics as the penetration depths increased. In addition to qualitative insights, in-situ testing is useful to extract quantitative information pertaining to the observed deformation modes/ mechanisms. Based on the load-displacement data, precise critical stresses $\left(\sigma_{\mathrm{cr}}\right)$ for the activation of specific mechanisms can be calculated. During indentation, effective stress is determined using force $(\mathrm{F})$ and the projected contact area $\left(A_{p}\right)$, using the following relationship ${ }^{13}$ : 

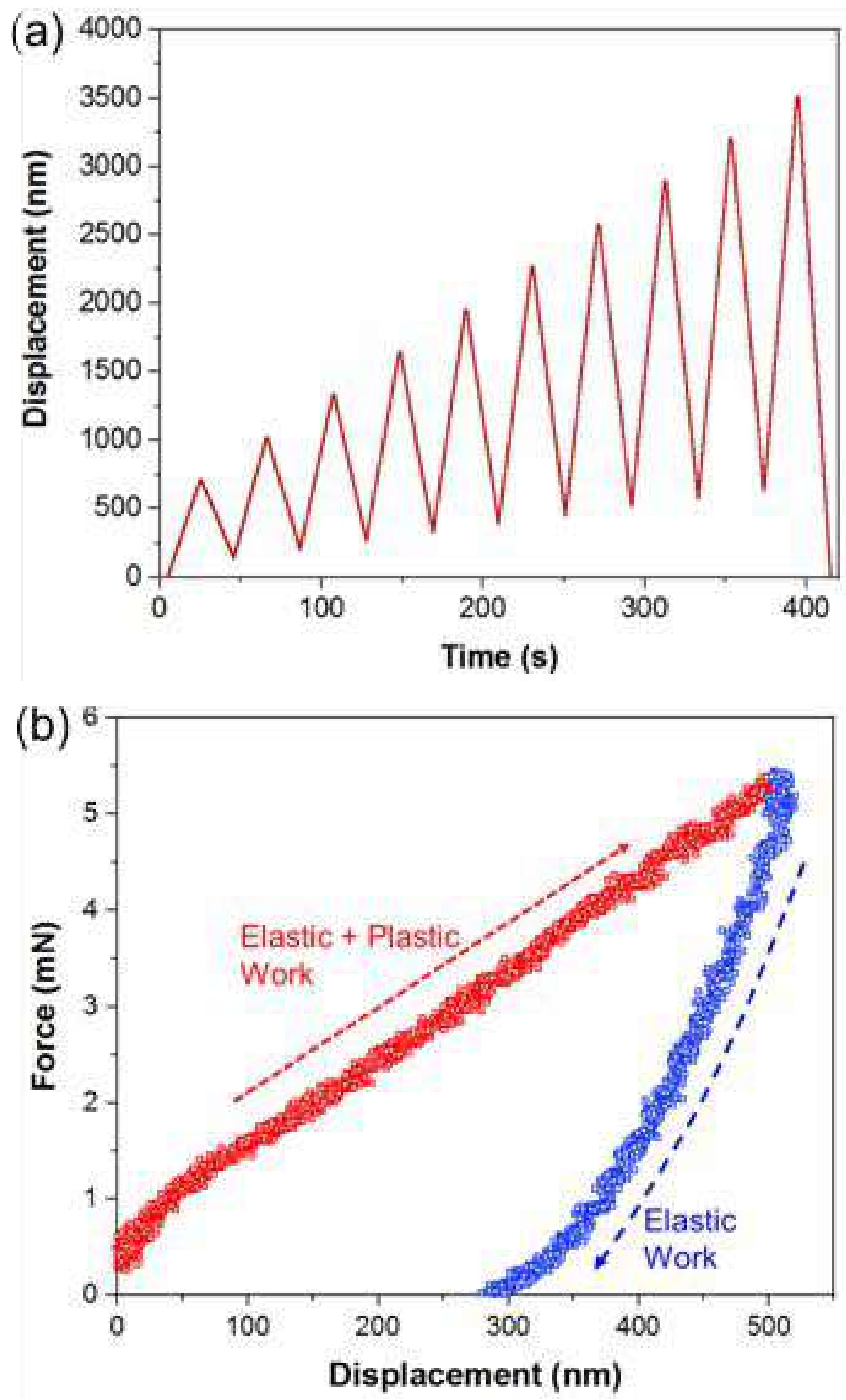

Figure 8.5. (a) Programmed loading cycles for in-situ testing of milled trenches in displacement-control mode, and (b) illustration of work expended and recovered in an indentation cycle. 


$$
\sigma=\frac{F}{A_{p}}
$$

where the projected contact area at a given indentation depth $(h)$ is equal to the crosssectional area of the indenter tip at that depth. For a pyramidal indenter geometry, the projected area of contact during conventional indentation test is expressed as ${ }^{14}$ :

$$
A_{p}=3 \sqrt{3} h^{2} \tan ^{2} \theta
$$

where $\theta$ is the face angle with the central axis of the indenter. In these investigations, a cube corner tip is used $\left(\theta=35.26^{\circ}\right)$. Further, the material removed during trench milling is accounted for by dividing the area equation above by a factor of 2 . The simplified form of the equation for $A_{p}$ is then obtained to be:

$$
A_{p}=1.3 h^{2}
$$

Therefore, critical stresses for specific deformation mechanisms activated during indentation loading of trenches are obtained using the simplified relationship:

$$
\sigma=\frac{F}{1.3 h^{2}}
$$

Cyclic tests with incrementally increasing displacements are highly informative as they can capture multiple mechanisms activated under different stress regimes, as the peak indentation displacements are varied from 500 to 3,500 nm (Figure 8.5a). The mechanisms discussed in Sections 8.2 and 8.3, such as pile-up, crack propagation, crack bridging and crack deflection involve non-recoverable dissipation of energy. Force-displacement data captured in conjunction with in-situ imaging allow for quantifying the mechanical work done for activating specific deformation modes in the hierarchical BNNT-MMC 
microstructures. The area under the force-displacement curve is a measure of mechanical work: while the area under the loading segment gives combined elastic-plastic work; the area under the unloading curve is a measure of elastic recovery or undissipated mechanical work $^{15}$ (Figure 8.5b). Therefore, the plastic work or the energy expended ${ }^{16}$ in a given indentation cycle $(i)$ can be determined by subtracting the elastic work from the total work:

$$
W_{i}=\int_{h_{\min , i}}^{h_{\max , i}} F d h-\left|\int_{h_{\max , i}}^{h_{r e s, i}} F d h\right|
$$

where $h_{\min }$ represents the indentation depth at the beginning of a loading cycle, $h_{\max }$ represents the peak penetration depth and $h_{\text {res }}$ represents the residual depth (permanent deformation) at the end of the given loading cycle, $i$.

It is noteworthy that the equation above is a measure of plastic work done in a single indentation cycle. Over multiple cycles (as shown in Figure 8.5a), the energy absorbed by the material will add up. Therefore, if a mechanism of interest (such as crack initiation or material pile-up) is seen in $n^{\text {th }}$ indentation cycle, the threshold energy absorption $\left(E_{T h}\right)$ for the activation of that mechanism will be equivalent to the cumulative work done up to that cycle:

$$
E_{T h}=\sum_{i=1}^{n} W_{i}
$$

The energy expended during the tests is a function of specimen volume being probed. To report representative energy dissipation values independent of material volume, we have defined a threshold specific energy parameter $\left(S E_{T h}\right)$, obtained by dividing $E_{T h}$ with the volume of the material displaced due to indentation loading. This volume $(V)$ can be determined from the projected area of contact and the indenter penetration depth: 


$$
V=A_{p} . h
$$

Substituting $A_{p}$ from Eq. (8.3) to Eq (8.7) above, the material volume removed can be expressed as a function of indentation depth, $h$ :

$$
V=1.3 h^{3}
$$

In order to determine the material removed at the end of a given indentation cycle, the value of residual depth $\left(h_{r e s}\right)$ for that cycle should be substituted in place of $h$ in Eq. (8.8). Therefore, the threshold specific energy $\left(S E_{T h}\right)$ can be calculated by the following relationship:

$$
S E_{T h}=\frac{\sum_{i=1}^{n} W_{i}}{1.3 h_{r e s, n}^{3}}
$$

The following sub-sections discuss different mechanisms observed during in-situ trench testing. The analytical framework discussed above is used for computing the critical stresses and the threshold specific energies required for activating these deformation mechanisms.

\subsubsection{Low-Temperature Sintered Composite}

\section{A. Inter-particle Crack Propagation}

In-situ imaging during indentation demonstrated crack initiation and propagation along the prior particle boundaries (Figure 8.6 and V8.4). This observation is in agreement with the mechanisms observed during meso-scale testing, where micro-porosities between the particles provided a preferred pathway for indentation-induced cracks (Figure 8.2a). The 

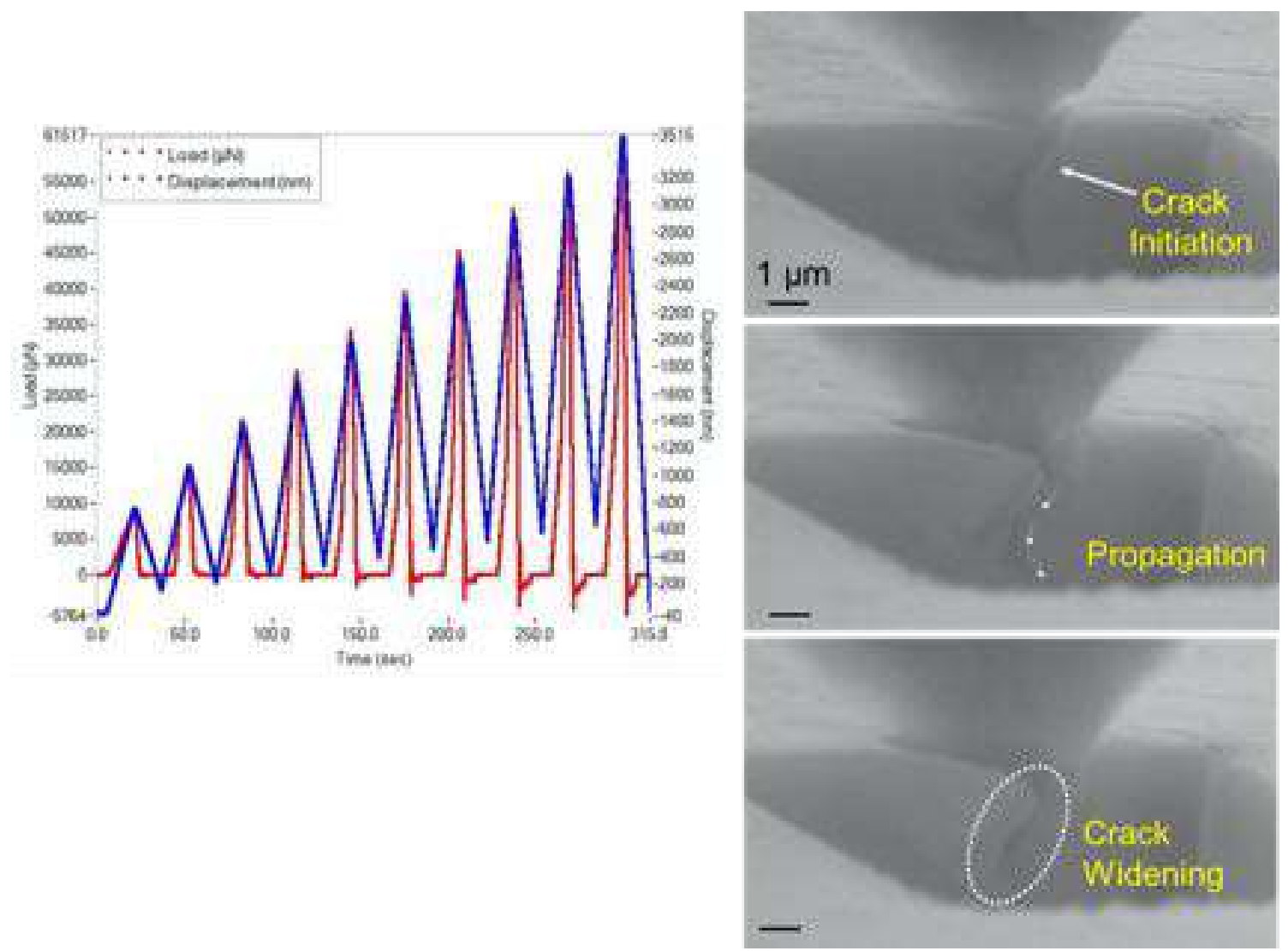

Figure 8.6. Force-displacement response and in-situ SEM images demonstrating crack initiation, propagation and widening along the alloy particle boundaries in LT composite.

crack initiation was observed in the $3^{\text {rd }}$ indentation cycle at $\sim 11.5 \mathrm{mN}$ applied load and a depth of $\sim 0.94 \mu \mathrm{m}$. This corresponds to the critical stress of $10 \mathrm{GPa}$ for inter-particle crack propagation. The specific energy expended for crack initiation was calculated to be $\sim 19.5$ $\mathrm{GJ} / \mathrm{m}^{3}$. In the subsequent loading cycles, the crack propagated along the particle boundaries, shown in Figure 8.6. Eventually, as the elastic-plastic deformation zone expanded due to deeper indentation, prominent widening or opening of cracks was observed $\left(8^{\text {th }}\right.$ cycle). The cumulative work done for crack widening was $\sim 33.3 \mathrm{~nJ}$, about 8 times the work expended for crack initiation. However, since the material removal volume 
is much higher for deeper indents, the net specific energy dissipation is lower for the crack opening mechanism $\left(\sim 4.4 \mathrm{GJ} / \mathrm{m}^{3}\right)$ as compared to crack initiation $\left(19.5 \mathrm{GJ} / \mathrm{m}^{3}\right)$. This is because significantly higher specific energy is required for breaking the bonds during crack initiation and propagation. Crack widening, on the other hand, is caused due to mechanical straining of the cracked region and does not require extensive bond breakage.

\section{B. Particle De-bonding and Push-out}

Some of the regions in the microstructure were susceptible to particle de-bonding due to indentation forces (V8.5). An SEM snapshot captured during the test shows the detachment of a particle under highly concentrated stresses exerted by the tip (Figure 8.7a). The critical stress for particle detachment is calculated to be $7.5 \mathrm{GPa}$. The de-bonding and detachment occurred in the third loading cycle, amounting to the total specific energy dissipation of $\sim 10.5 \mathrm{GJ} / \mathrm{m}^{3}$. This value is half the energy expended to initiate inter-particle cracks discussed in Figure 8.6. This suggests the particle de-bonding mechanism is different from inter-particle crack propagation. While the former arises due to weak or incomplete particle-particle bonding; the latter is driven by the enlargement and coalescence of micropores. The particle de-bonding was not as straightforward in some other cases. One of the tests revealed a progressive sliding of a particle due to indentation loading, as shown in Figure 8.7b. As the indentation depth increased from $\sim 1.4 \mu \mathrm{m}$ to $2.7 \mu \mathrm{m}$, one of the particles under the probe underwent re-alignment by almost $45^{\circ}$. Further penetration to 3 $\mu \mathrm{m}$ caused the particle to lift off and slide out of its original configuration (V8.6). These

findings suggest non-homogeneous bonding in LT-composite. Some of the regions in the 
(a)

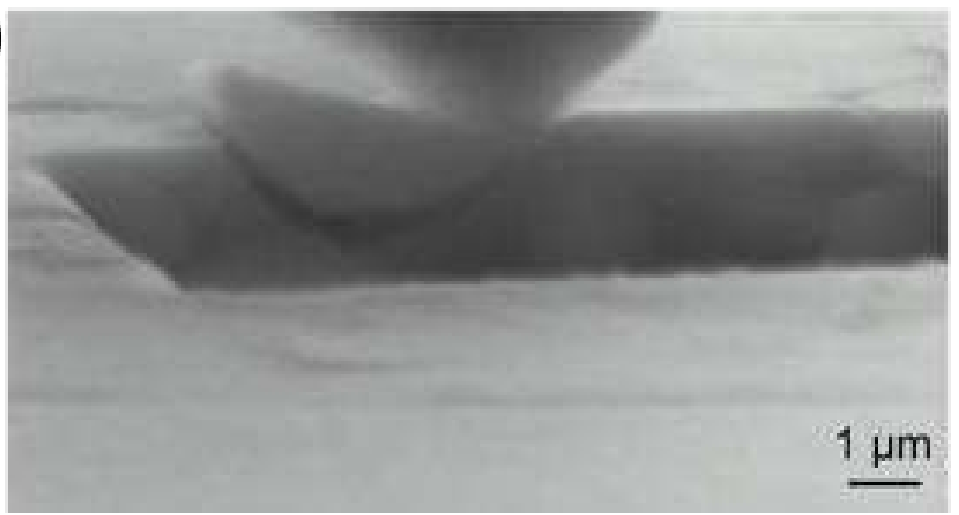

(b)

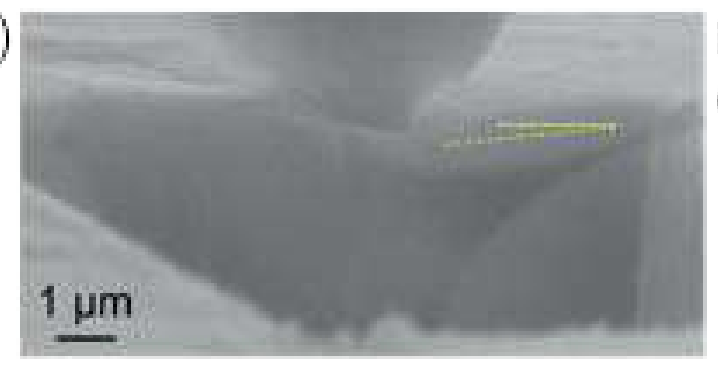

\section{Depth}

$(\mu \mathrm{m})$
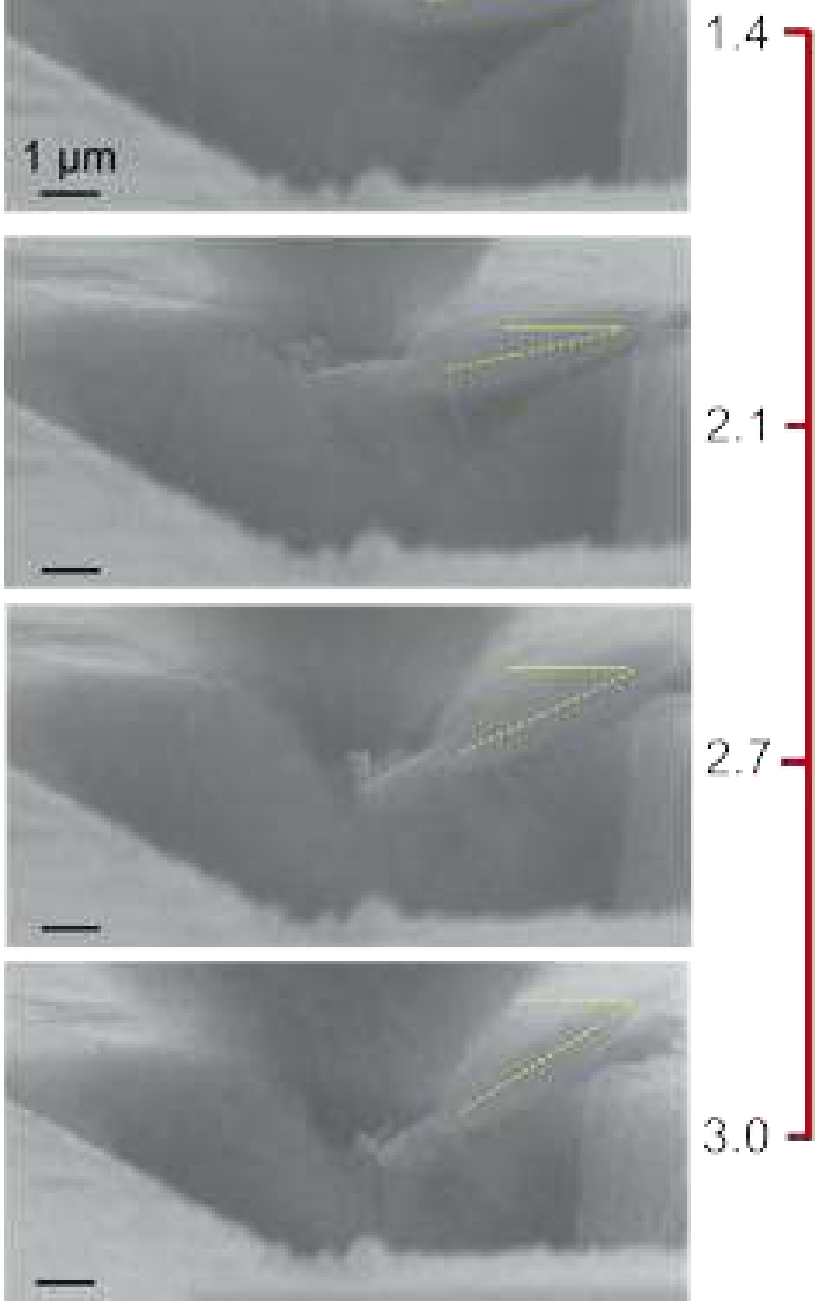

$3.0]$

Figure 8.7. (a) SEM micrograph showing particle detachment due to mechanical loading, and (b) imaging at multiple indentation depths reveal transition from intrinsic plasticity to inter-particle de-bonding deformation in LT-Ti6Al-BNNT. 
microstructure are susceptible to failure initiation. De-bonding can be either direct detachment or plasticity-driven sliding along particle interfaces.

\section{Plastic Flow}

Plastic deformation was the dominant deformation mechanism in LT composite (V8.7). The real-time SEM images during a cyclic indentation test at different loads and displacements are shown in Figure 8.8a. Localized loading drove the plastic flow of material underneath the indenter tip. The deformation zone was expanded with progressive

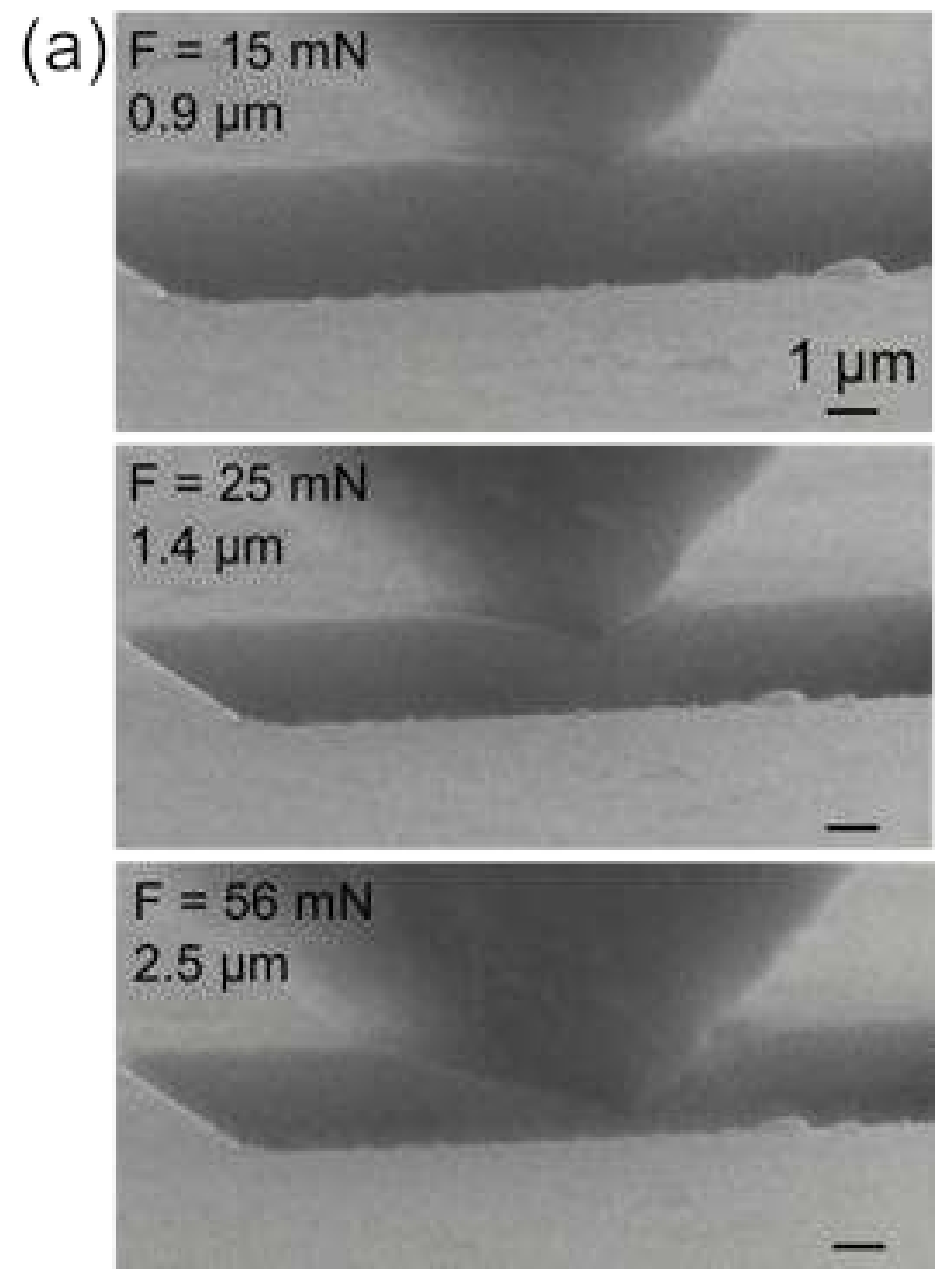




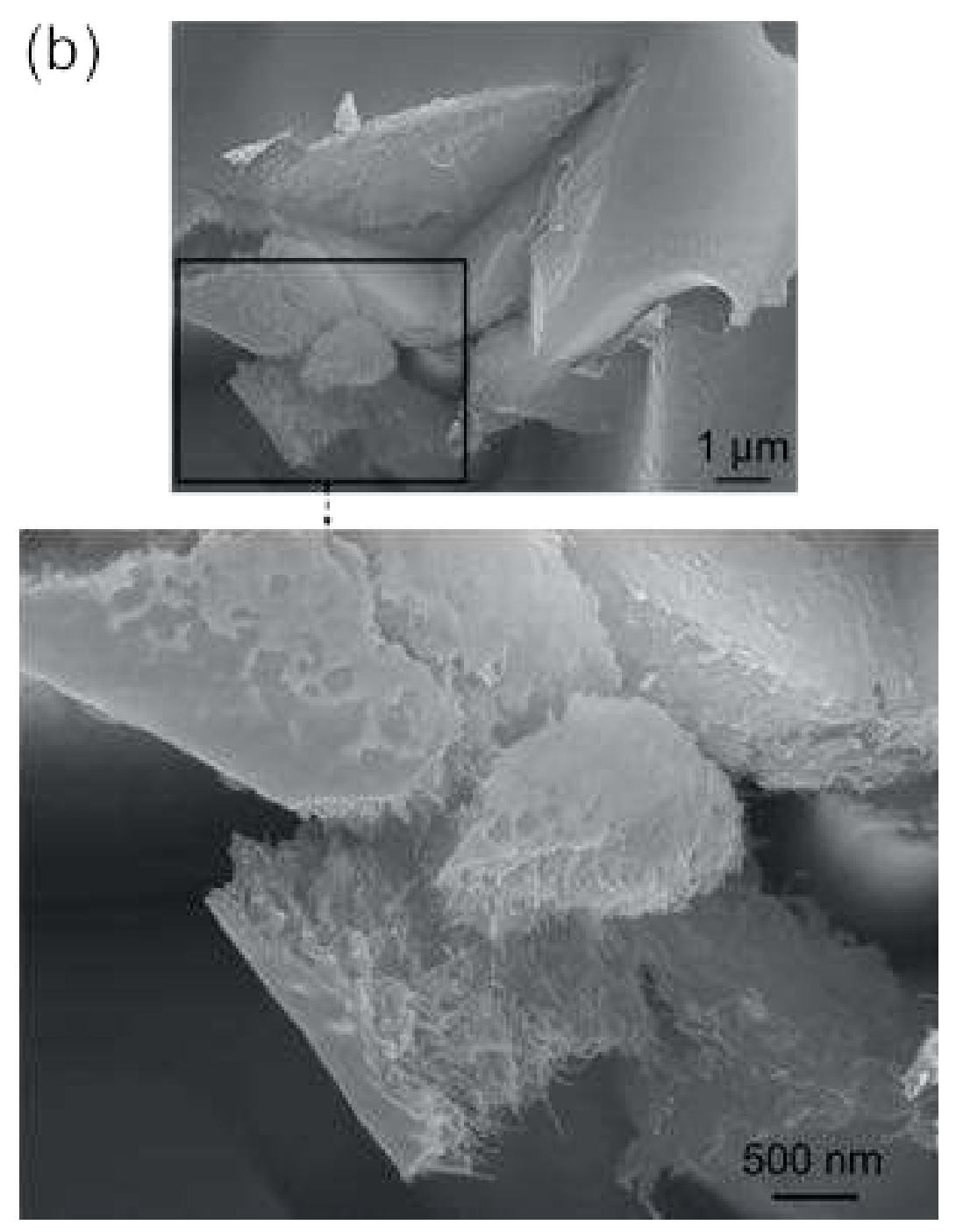

Figure 8.8. (a) Real-time SEM images reveal the plastic flow of material without cracks or particle de-bonding, and (b) SEM images of a separate region where excess clusters resulted in poor adhesion and catastrophic failure.

tip penetration. However, there were no local cracks observed or inter-particle sliding. This observation suggests that not all particle interfaces are susceptible to crack initiation and de-bonding events. The local variation in mechanisms is indicative of non-uniform bonding in the sintered composite. The degree of bonding is greatly affected by BNNTs. Excess nanotubes can hinder inter-particle adhesion during sintering. The regions with 
predominantly plastic behavior (as demonstrated in Figure 8.8a) are free of BNNT clusters. On the other side of the spectrum, BNNT accumulation can lead to catastrophic failure under indentation loading. Such an observation was made in one of the in-situ tests (post-failure images shown in Figure 8.8b). Therefore, the effective dispersion of nanotubes in the matrix seems to promote the plastic deformation mechanism in the composite.

\subsubsection{High-Temperature Sintered Composite}

\section{A. Shear Band Propagation}

Sub-surface imaging demonstrated indentation-induced shear banding in HT-composite (Figure 8.9 and V8.8). In the first loading cycle, the formation of the shear band was observed under the tip at a shallow indentation depth of $\sim 700 \mathrm{~nm}$ (Figure 8.9a). In subsequent cycles, as the penetration depth exceeded $1.5 \mu \mathrm{m}$, shear band propagation was observed. Figure 8.9b shows an SEM micrograph at the end of the $4^{\text {th }}$ loading cycle, and long-range propagation of shear bands emanating from the region underneath the probe can be seen. This long-range plastic flow is promising from the standpoint of retaining ductility despite the presence of ceramic nanotubes. The cumulative energy expended up to the onset of pronounced shear band propagation is calculated to be $33.9 \mathrm{~nJ}$. This corresponds to a specific energy dissipation of $\sim 8 \mathrm{GJ} / \mathrm{m}^{3}$. Energy absorption by shear band propagation will offset or minimize catastrophic cracking during the mechanical loading of the composites. 


\section{Indentation-induced Shear Banding

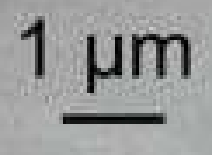

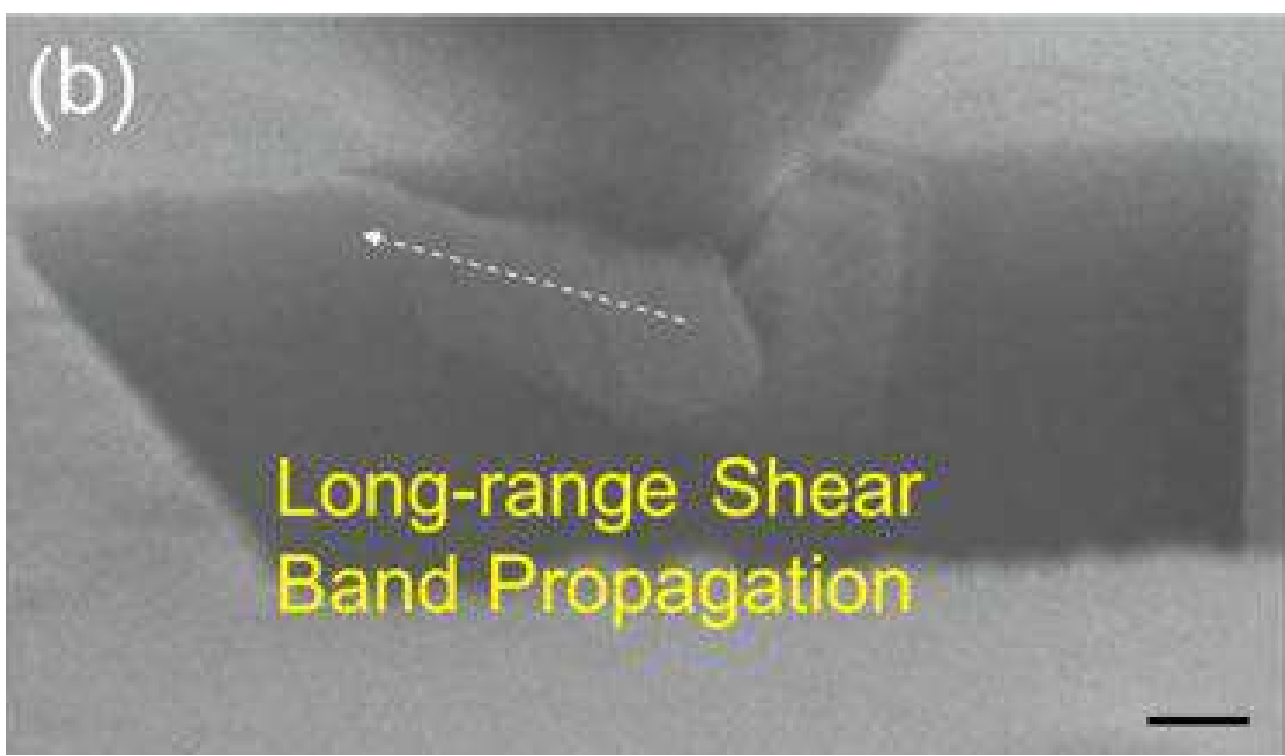

Figure 8.9. In-situ indentation showing onset of shear banding (a), and log-range shear band propagation with subsequent loading cycles (b).

\section{B. Crack Propagation}

In addition to plastic deformation, crack initiation and propagation was observed in the deformation zone beneath the tip (V8.9). Unlike LT composite where cracking was observed along the particle interfaces; here the propagation seems to be transgranular in 
nature (Figure 8.10). This is indicative of stronger particle-particle bonding during HTsintering. Transgranular cracking also suggests that ceramic phases play a prominent role during the deformation of HT-composite. The ceramic phases are susceptible to cracking. Nevertheless, the forces required to trigger cracking are much higher in HT composite. Figure 8.10 shows the cracking is initiated in the $7^{\text {th }}$ indentation cycle, and at a critical load and depth of $\sim 90 \mathrm{mN}$ and $2.2 \mu \mathrm{m}$, respectively. This corresponds to a critical stress value of $14.3 \mathrm{GPa}$, which is over $4 \mathrm{GPa}$ higher than the critical stress calculated for LT composite (Figure 8.6). A cumulative work of $71.97 \mathrm{~nJ}$ is expended up to crack initiation point, P1. Between P1 and P2, the crack is seen to propagate under continued indentation loading, requiring an additional $22.4 \mathrm{~nJ}$. The specific energy absorbed for crack initiation in HT composite is calculated to be $11.3 \mathrm{GJ} / \mathrm{m}^{3}$. It is noteworthy that the cracking mechanism in HT composite is observed in the $7^{\text {th }}$ loading cycle, as opposed to the $3^{\text {rd }}$ loading cycle in LT composite. As a result, the mechanical work is dissipated over a much larger volume of material in HT composite, yielding a relatively lower $S E_{T h} \approx 11.3 \mathrm{GJ} / \mathrm{m}^{3}$ in comparison to $\mathrm{LT}$ composite $\left(S E_{T h} \approx 19.5 \mathrm{GJ} / \mathrm{m}^{3}\right)$. Higher energy dissipation per unit volume in the LT composite can be ascribed to inter-particle sliding because of comparatively weaker bonding observed in Figure 8.7. These inter-particle sliding mechanisms are absent in HT composite, which explains the lower $S E_{T h}$ value. The elastic-plastic deformation zone expands as the indentation test progresses. This results in stress concentration in the periphery of the trench, triggering additional cracking at indentation depths exceeding 2.6 $\mu \mathrm{m}(\mathrm{P} 3)$. 

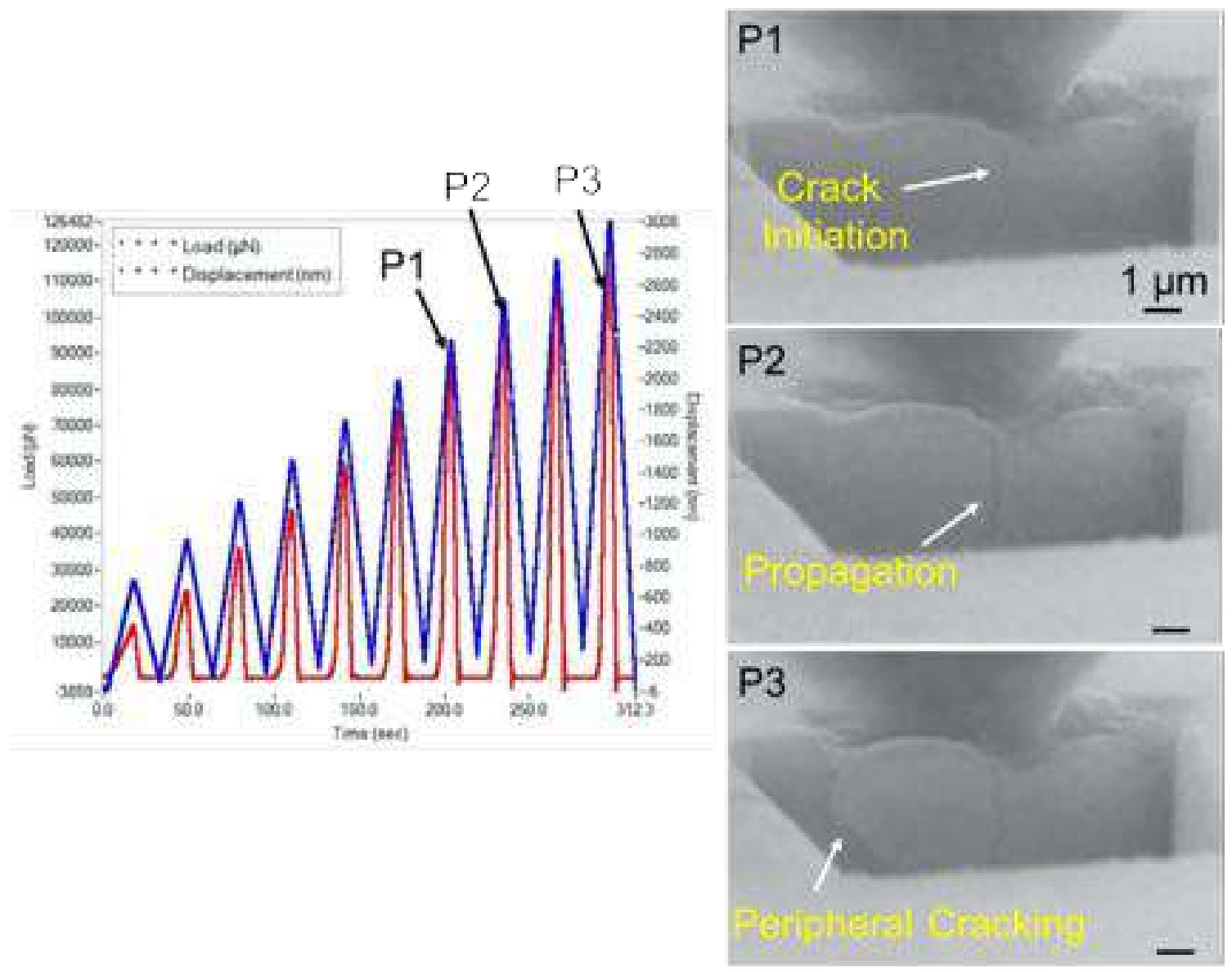

Figure 8.10. Force-displacement response and in-situ SEM images showing time-resolved crack initiation and propagation in HT-sintered Ti6Al4V-BNNT.

\section{Ductile Cracking}

There were some regions in the microstructure where cracking was accompanied by the plastic flow of material under indentation loading (V8.10). Figure 8.11a demonstrates a tortuous crack propagating due to indentation. Multiple deflection events are observed under the tip. This finding is in agreement with extensive deflection observed during mesoscale testing (Figure 8.2b). In subsequent indentation cycles, crack propagation induced local plasticity in a narrow zone under the tip (Figure 8.11b). This observation is in striking 

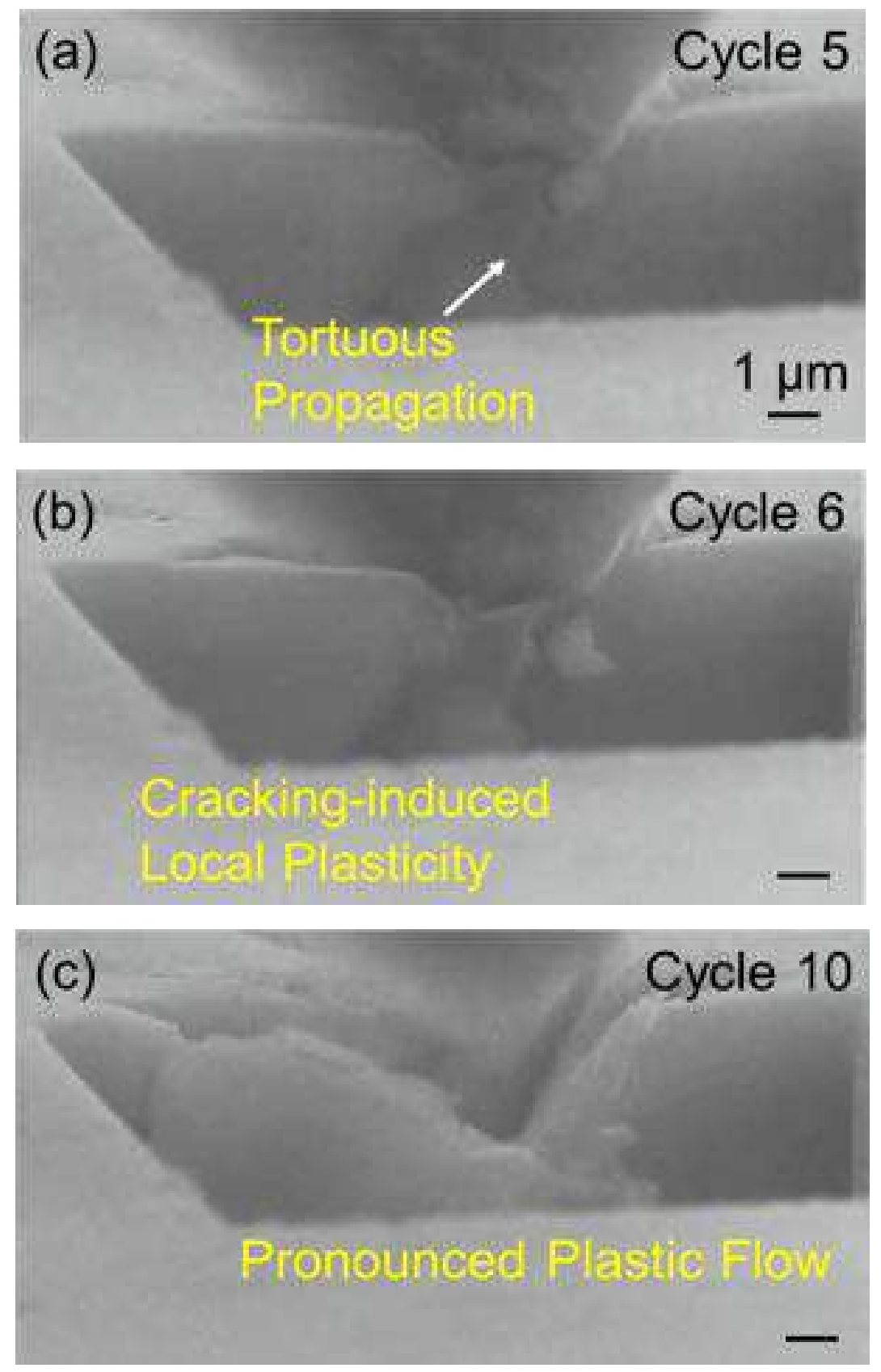

Figure 8.11. In-situ SEM images showing the transition from tortuous crack propagation (a), to local plasticity (a), to pronounced plastic flow of material (c).

contrast to brittle-style crack propagation seen in Figure 8.10. With further indentation loading, pronounced plastic deformation and material removal are observed under the 
probe (Figure 8.11c). The critical stress for ductile cracking is calculated to be $\sim 11 \mathrm{GPa}$, somewhat lower than brittle transgranular cracking $\left(\sigma_{\mathrm{cr}}=14.3 \mathrm{GPa}\right)$. The cumulative energy dissipation leading up to crack initiation (in the $5^{\text {th }}$ loading cycle) was found to be $40.3 \mathrm{~nJ}$, lower than the energy expended for brittle cracking (71.97 $\mathrm{nJ})$. Brittle cracks require relatively higher stresses and energies because the bond strength in ceramics is inherently higher. The energy per unit volume for ductile cracking is $\sim 6.5 \mathrm{GJ} / \mathrm{m}^{3}$, which is comparable to the shear banding mechanism shown in Figure 8.9. The ductile crack propagation is likely to be prominent in the regions where ceramic phases are discretely distributed in the alloy. Intermittent deflection events occur when a crack propagating in the alloy encounters these nanoscale ceramic species. Ceramic phases are typically stronger and require higher energy for bond cleavage, causing the crack to deflect.

\subsubsection{Reinforcement Efficacy of Deformation Mechanisms}

The critical stresses, threshold energies and specific energies for each of the mechanisms discussed above are summarized in Table 8.1. In order to evaluate the reinforcement efficacy of nanotubes and interphases, the critical stresses $\left(\sigma_{c r}\right)$ for the activation of the aforementioned mechanisms are divided by the hardness $(H)$ of pristine, unreinforced Ti6Al4V. The reason for choosing hardness as a reference parameter is because of identical stress-states during hardness measurements and in-situ trench tests carried out in this work. The value of $H$ was determined to be 7.8 GPa by indentation experiments in Section 8.3. A comparison of $\left(\sigma_{c r} / H\right)$ for different mechanisms in Table 8.1 shows that inter-particle cracking, transgranular cracking as well as ductile cracking are activated at stresses 
exceeding the hardness of the alloy. These calculations indicate that regions with poor inter-particle bonding are deeply concerning, as they can detach under stresses lower than the hardness of the pure alloy $\left(\sigma_{c r} / H<1\right)$. This will forfeit the purpose of adding BNNTs, as de-bonding events may initiate premature failure in the composite. Local in-situ investigations suggest that the poorly bonded sites in the microstructure are limited, and it's not a pervasive issue throughout the microstructure. The remedy to overcome the issue is to prevent BNNT clustering so that the powder particles can effectively bond during sintering. Another noteworthy observation is that the specific energy absorption value is lowest for plastic deformation mechanisms, like shear band propagation and ductile cracking. Therefore, plastic deformation will be energetically favored over other mechanisms when a composite is subjected to bulk- or macro-scale loading.

Table 8.1. Comparison of stress and energy parameters associated with deformation mechanisms observed via in-situ investigations.

\begin{tabular}{lllll}
\hline Mechanism & $\begin{array}{l}\text { Critical } \\
\text { Stress } \\
(\mathrm{GPa})\end{array}$ & $\begin{array}{l}\text { Reinforcement } \\
\text { Efficacy, } \sigma_{c r} / H\end{array}$ & $\begin{array}{l}\text { Threshold } \\
\text { Energy } \\
(\mathrm{nJ})\end{array}$ & $\begin{array}{l}\text { Specific } \\
\text { Energy (GJ } \\
\left.\mathrm{m}^{-3}\right)\end{array}$ \\
\hline Inter-particle & 10 & 1.3 & 4.48 & 19.5 \\
Cracking & 14.3 & 1.8 & 71.97 & 11.3 \\
\hline Transgranular & & & & \\
Cracking & 11 & 1.4 & 40.3 & 6.5 \\
\hline Ductile Cracking & 7.5 & 0.96 & 4.51 & 10.5 \\
\hline Particle De-bonding & & - & 33.9 & 8 \\
\hline Shear Band & - & & & \\
Propagation & & & & \\
\hline
\end{tabular}




\subsection{Correlation between Deformation Mechanisms and Interface Chemistry}

In-situ trench tests discussed in Section 8.4 provide useful insights about sub-surface mechanisms. The imaging magnifications are chosen to ensure the field of view is broad enough to capture crack propagation and plastic flow (shear banding) mechanisms. These mechanisms are active in micrometer length scales. Therefore, the magnification is chosen such that the image frames are $\sim 5-15 \mu \mathrm{m}$ wide (Figures 8.6-8.10). It is not possible to resolve individual nanotubes or nano-phases in the microstructure at these magnifications. Therefore, high-magnification SEM imaging was performed after the tests to decipher the role of nanotubes and interphases in influencing the mechanical properties. Figure 8.12 shows SEM images of the indentation deformation zone. One of the salient observations was extensive pull-out and bridging action due to long nanotubes in LT-composite (Figure 8.12a). The images highlight that long BNNTs resist material removal and plastic flow during the test. In the case of HT composite, the bridging action was not as prominent and fewer long BNNTs are observed via SEM imaging (Figure 8.12b). Instead, the pull-out of shorter needle-like features is seen in the deformed regions. The needle-like features have

been identified as titanium boride in previous studies on Ti-BNNT composites ${ }^{8,12}$. This observation suggests the strengthening behavior is nanotube-dominated in LT-composite and interphase-dominated in HT-composite. 

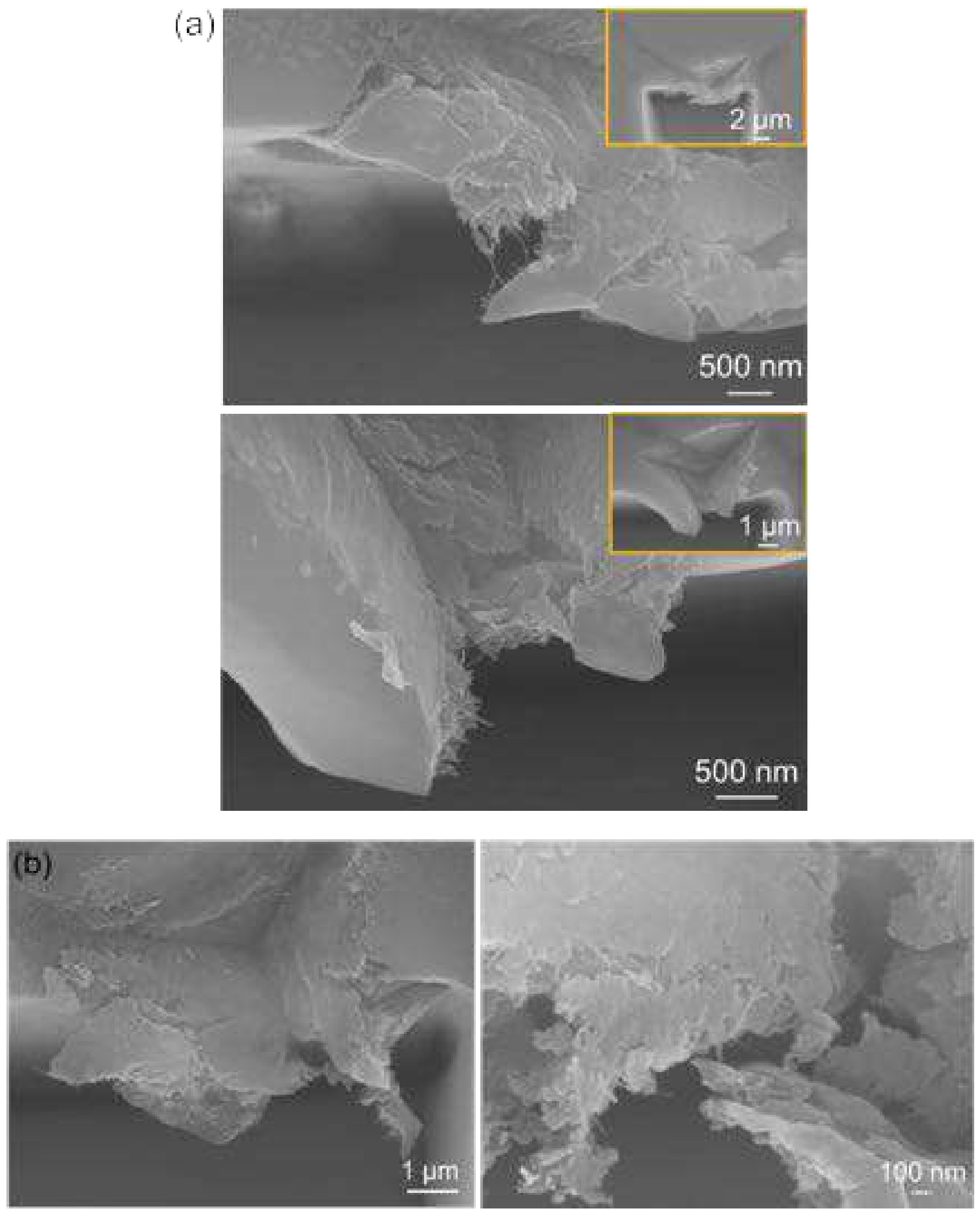

Figure 8.12. SEM imaging after indentation tests reveals crack bridging and pull-out of long nanotubes in LT-sintered composite (a) and needle-like nano-species in HT-sintered composite (b). 


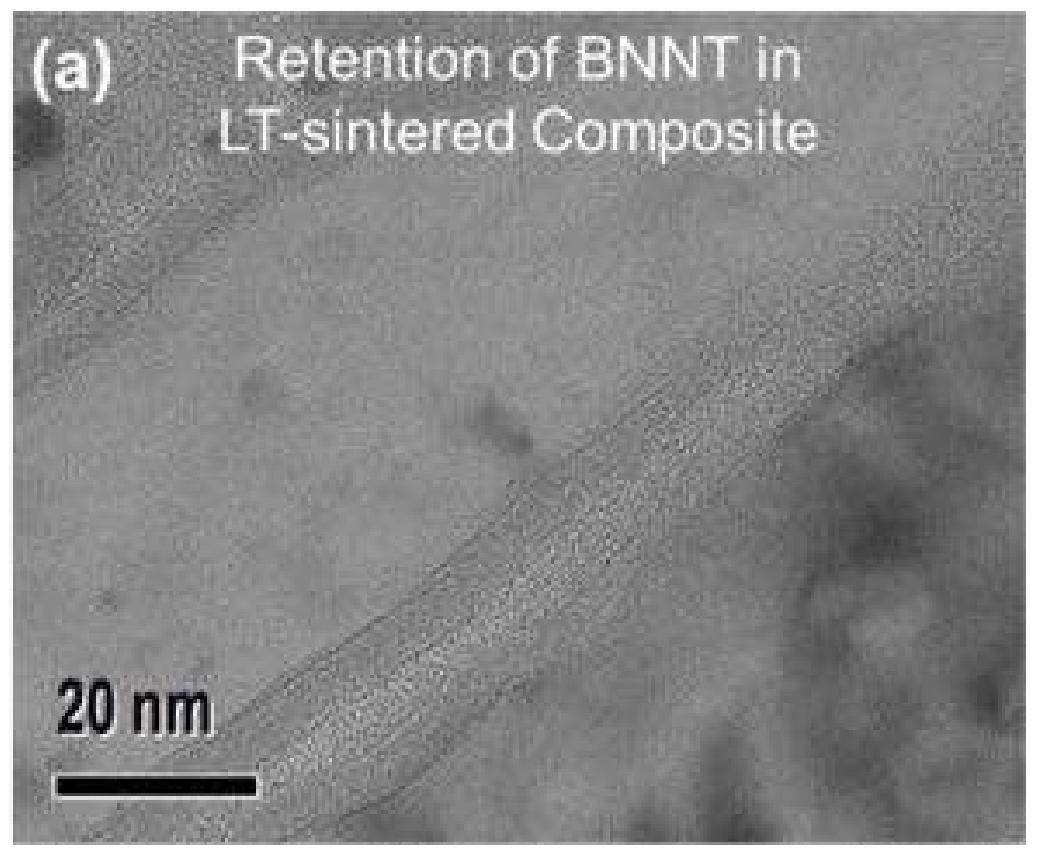

(b) Interface Chemistry in LT-sintered Ti6Al4V-BNNT
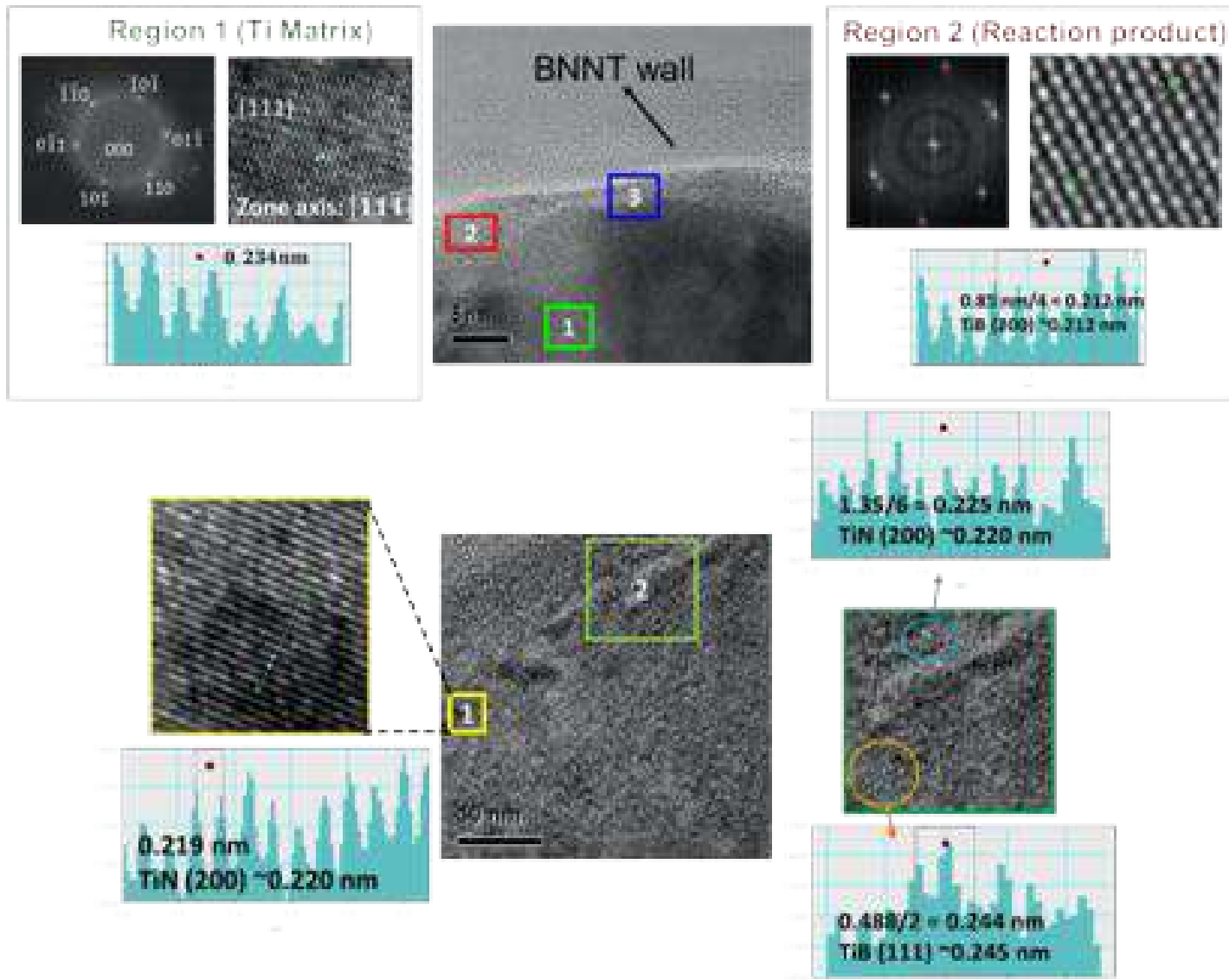


\section{(c) Interface Chemistry in HT-sintered Ti6Al4V-BNNT}

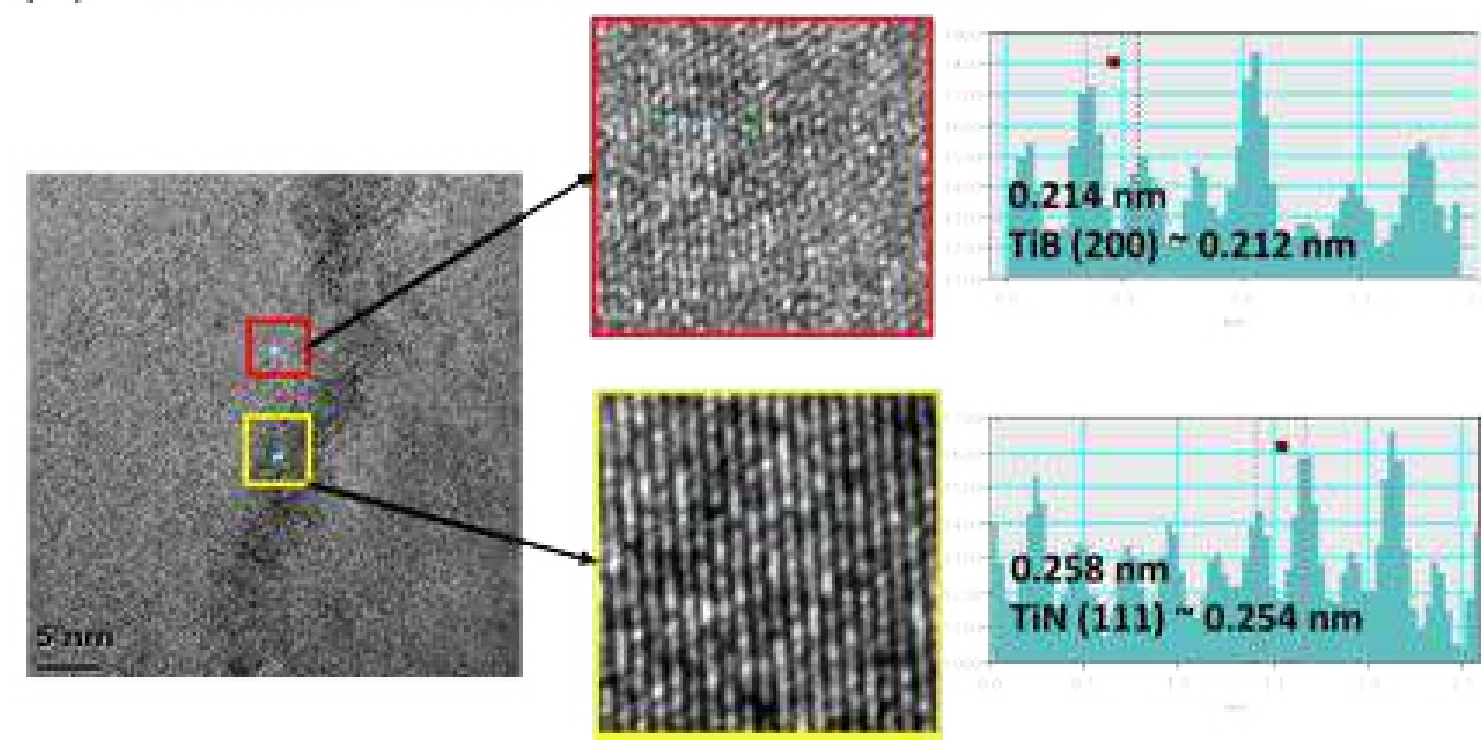

Figure 8.13. HRTEM investigation of Ti6Al4V-BNNT composites showing retention of long nanotubes in LT-sintered composite (a), co-existence of BNNT with TiN and TiN interphases in LT-sintered composite (b), and significant reactions/ phase transformations in HT-sintered composite (c).

These differences in the deformation mode exhibited by LT and HT composites were correlated with interface chemistry by TEM analysis. HRTEM imaging confirmed the retention of long BNNTs in the composite (Figure 8.13a). Fast Fourier transform (FFT) analysis at the BNNT-Ti alloy interface revealed the formation of $\mathrm{TiN}$ and $\mathrm{TiB}$ in the LT composite (Figure 8.13b). The reactions are controlled and the nanotube structure is retained, as seen from the micrograph. Contrary to this, the presence of reaction products was more prominent in HT composite. Figure 8.13c shows the formation of $\mathrm{TiB}$ and $\mathrm{TiN}$ nano-phases in the microstructure. The retention of long, pristine and undamaged BNNTs could not be confirmed by TEM analysis. This finding supports the prevalence of nanotube-dominated strengthening in LT composite (Figure 8.12a) and interphase- 
dominated strengthening in HT composite (Figure 8.12b). It is expected that while long nanotubes provide crack-bridging ability; ceramic interphases promote matrix-to-filler load transfer ${ }^{11,17}$ and result in stiffening and hardening due to the presence of hard phases in the microstructure ${ }^{4}$. The HRTEM findings explain the meso-scale and micro-scale indentation measurements discussed in Sections 8.2 and 8.3, where HT composite exhibited higher hardness and stiffness than the LT composite.

TEM analysis is highly informative to resolve individual phases, study their morphologies with extraordinary resolution, identify the atomic specific planes and investigate the interface between the matrix and the nanotube. However, it's not feasible to statistically comment on the extent of chemical transformations because the micrographs barely capture a few nanotubes at best. To analyze the distribution of the nano-phases in the microstructure, XPS analysis of a relatively larger area $(100 \mu \mathrm{m}$ by $100 \mu \mathrm{m})$ was performed. Fitting of N1s and B1s spectra confirmed the formation of $\mathrm{TiN}, \mathrm{TiB}$ and $\mathrm{TiB}_{2}$ in both LT and HT composites (Figure 8.14a). Retention of BN was also confirmed in both the composites, suggesting not all the nanotubes undergo reactive transformations during sintering. Additionally, B-N-O bonding interaction was identified, indicative of the attachment of oxygen-based functional groups to BNNT during the purification step.

In addition to the identification of bonding interactions, atomic percentage profiles were created to visualize the spatial distribution of the nano-species (Figure 8.14b). The images were superimposed to clearly compare the fraction and distribution of interfacial products (borides and nitrides) with respect to $\mathrm{BN}$. The overlaid images corresponding to B1s show limited formation of borides (green colored species) in LT composite. However, 

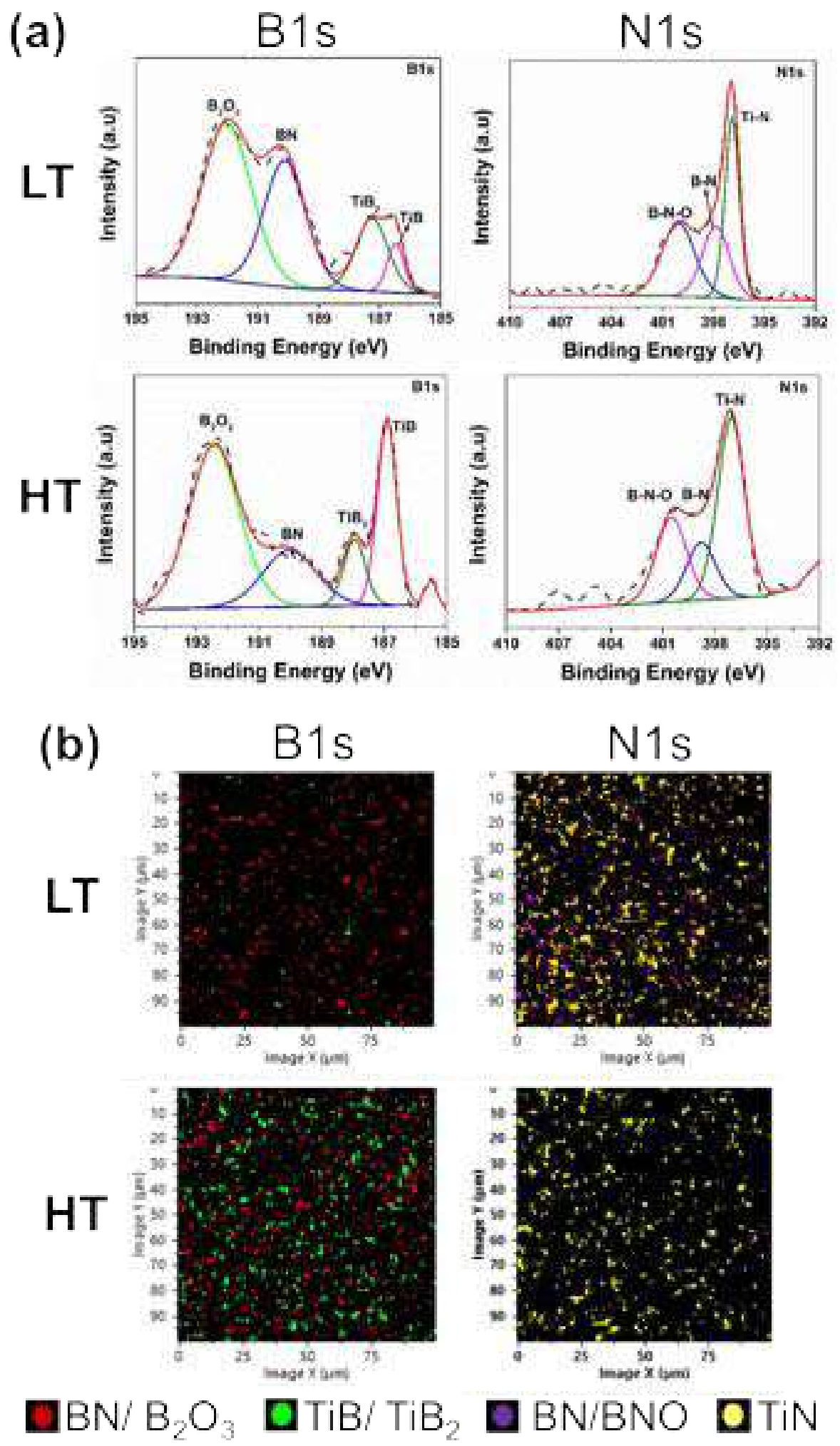

Figure 8.14. (a) XPS B1s and N1s spectra for LT and HT composites, and (b) micro-scale chemical mapping of the composite microstructure with superimposed images comparing the relative atomic fraction of $\mathrm{BN}$ and interphases. 
the boride phases are markedly increased in HT composite. Image analysis revealed a jump in area fraction occupied by boride species from $\sim 2.9 \%$ in LT composite to $~ 16.3 \%$ in HT composite. The superimposed images for N1s spectra facilitate the comparison between $\mathrm{BN}$ and TiN phases. The area fraction of $\mathrm{BN}$ (purple colored species) was calculated to drop from $\sim 7.3 \%$ in LT composite to $\sim 3.2 \%$ in HT composite. These findings provide statistical evidence of enhanced phase transformations during HT sintering. In order to quantify the extent of reactions between the matrix and the nanotubes, we have defined an interface reaction index $\left(I_{R}\right)$, which compares the area fraction of interfacial products $\left(f_{I P}\right)$ with respect to the original filler material $\left(f_{F}\right)$ :

$$
I_{R}=\frac{f_{I P}}{f_{F}}
$$

Based on the images shown in Figure 8.14b, the interface reaction indices were computed separately for borides $\left(I_{R, B}\right)$ and nitride $\left(I_{R, N}\right)$ interphases. The value of $I_{R, B}$ shot up from 0.25 for LT composite to 0.65 for HT composite. $I_{R, N}$ also leaped from 3.65 (LT composite) to 5.09 (HT composite). These calculations indicate TiN is the major interphase in the composites studied in this work. The difference in the fraction of borides and nitrides is most striking in LT composite, based on the spatial distribution images in Figure 8.14b. However, the formation of borides is accentuated during HT sintering and the maps shown in Figure 8.14b demonstrate a comparable fraction of borides and nitride in the composite microstructure.

High-resolution chemical evaluation is extremely informative in understanding the in-situ mechanisms at the microstructure length scales discussed in this chapter. It can be concluded that the interphases observed in the composite microstructure positively 
contribute towards the stiffening and hardening of Ti6Al4V. Future studies should explore the role of interphases on the tensile deformation of this composite.

\subsection{Summary}

Interfacial reactions during the sintering of Ti6Al4V-BNNT composites can be controlled by tweaking the processing temperature. In-situ mechanical investigations revealed plastic flow, shear band propagation, cracking and crack-bridging as the prominent deformation mechanisms activated in the composite. Theoretical calculations suggest plastic deformation mechanisms are energetically favored, with the least specific threshold energy requirement of $6.5-8 \mathrm{GJ} \mathrm{m}^{-3}$, as opposed to brittle ceramic cracking which requires threshold energies exceeding $11 \mathrm{GJ} \mathrm{m}^{-3}$. This finding is promising to engineer BNNTMMCs with reasonable ductility. High-resolution imaging demonstrated crack bridging and crack deflection events due to nanotubes and interphases. Critical stresses required to initiate cracks in the composites are 1.3 to 1.8 times the hardness of the pristine alloy, confirming the reinforcement potential of ceramic nanophases. However, poor bonding and local clustering in some regions of the microstructure can lead to failure initiation at lower stresses. These issues can be remedied by improving nanotube dispersion and modifying sintering conditions. The extent of chemical reactions during sintering is quantified by high-resolution chemical mapping by XPS, confirming that the deformation behavior is BNNT-dominated in LT composite, whereas it is interphase dominated in HT-composite. The fundamental mechanistic insights obtained from this chapter will be helpful in guiding the processing and microstructure design of BNNT-MMCs. 


\section{References}

1. Lahiri, D. et al. Boron nitride nanotubes reinforced aluminum composites prepared by spark plasma sintering: Microstructure, mechanical properties and deformation behavior. Mater. Sci. Eng. A 574, 149-156 (2013).

2. Yamaguchi, M., Meng, F., Firestein, K., Tsuchiya, K. \& Golberg, D. Powder metallurgy routes toward aluminum boron nitride nanotube composites, their morphologies, structures and mechanical properties. Mater. Sci. Eng. A 604, 9-17 (2014).

3. Xue, Y. et al. Aluminum matrix composites reinforced with multi-walled boron nitride nanotubes fabricated by a high-pressure torsion technique. Mater. Des. 88, 451-460 (2015).

4. Singhal, S. K., Srivastava, A. K., Pasricha, R. \& Mathur, R. B. Fabrication of AlMatrix Composites Reinforced with Amino Functionalized Boron Nitride Nanotubes. J. Nanosci. Nanotechnol. 11, 5179-5186 (2011).

5. Nautiyal, P. et al. Oxidative Unzipping and Transformation of High Aspect Ratio Boron Nitride Nanotubes into 'white Graphene Oxide' Platelets. Sci. Rep. 6, 29498 (2016).

6. Golberg, D., Bando, Y., Kurashima, K. \& Sato, T. Synthesis and characterization of ropes made of BN multiwalled nanotubes. Scr. Mater. 44, 1561-1565 (2001).

7. Chen, Y., Zou, J., Campbell, S. J. \& Caer, G. Le. Boron nitride nanotubes: Pronounced resistance to oxidation. Appl. Phys. Lett. 84, 2430-2432 (2004).

8. Bustillos, J. Boron Nitride Nanotube Reinforced Titanium Composite with Controlled Interfacial Reactions by Spark Plasma Sintering. (Florida International University, 2019).

9. Bustillos, J., Zhang, C., Loganathan, A., Boesl, B. \& Agarwal, A. Ultralow Temperature Densification of a Titanium Alloy by Spark Plasma Sintering. $A d v$. Eng. Mater. 2000076 (2020). doi:10.1002/adem.202000076

10. Zhou, W., Yamaguchi, T., Kikuchi, K., Nomura, N. \& Kawasaki, A. Effectively enhanced load transfer by interfacial reactions in multi-walled carbon nanotube reinforced Al matrix composites. Acta Mater. 125, 369-376 (2017).

11. Bakshi, S. R. \& Agarwal, A. An analysis of the factors affecting strengthening in carbon nanotube reinforced aluminum composites. Carbon N. Y. 49, 533-544 (2011). 
12. Bhuiyan, M. M. H., Li, L. H., Wang, J., Hodgson, P. \& Chen, Y. Interfacial reactions between titanium and boron nitride nanotubes. Scr. Mater. 127, 108-112 (2017).

13. Goodall, R. \& Clyne, T. W. A critical appraisal of the extraction of creep parameters from nanoindentation data obtained at room temperature. Acta Mater. 54, 5489-5499 (2006).

14. C. Fisher-Cripps, A. Nanoindentation. (Springer, 2005). doi:10.1007/978-1-44199872-9

15. Nautiyal, P., Mujawar, M., Boesl, B. \& Agarwal, A. In-situ mechanics of 3D graphene foam based ultra-stiff and flexible metallic metamaterial. Carbon N. $Y$. 137, 502-510 (2018).

16. Jha, K. K., Zhang, S., Suksawang, N., Wang, T. Lo \& Agarwal, A. Work-ofindentation as a means to characterize indenter geometry and load-displacement response of a material. J. Phys. D. Appl. Phys. 46, 415501 (2013).

17. Agarwal, A., Bakshi, S. R. \& Lahiri, D. Carbon Nanotubes: Reinforced Metal Matrix Composites. (CRC Press, Taylor \& Francis Group, 2011). 


\section{CHAPTER 9}

\section{SUMMARY, CRITICAL APPRAISAL AND FUTURE OUTLOOK}

This dissertation establishes chemical and structural stability of BNNTs at elevated temperatures $\left(>700^{\circ} \mathrm{C}\right)^{1,2}$, which is vital to engineer metal matrix composites. There were two key foci of the dissertation: (i) scalable processing and (ii) mechanical behavior of lightweight BNNT-MMCs. The work explored the reinforcement of $\mathrm{Al}, \mathrm{Mg}$, and $\mathrm{Ti}$ matrices using high-aspect-ratio nanotubes. While the investigations on Al were intended to probe microstructure evolution in an array of processing techniques ${ }^{3-5}$ for composite synthesis; the dissertation also examined $\mathrm{AZ} 31^{6}$ and Ti6Al4V matrices to explore the reinforcement potential of BNNT for other low-density structural metals.

Three different classes of processing techniques were used in this work: powder metallurgy (spark plasma sintering-solid state), equilibrium solidification (casting-liquid state), and additive manufacturing (plasma spray-semi solid state). These approaches, in conjunction with pre- and post-processing steps, were harnessed to design nanocomposite microstructures with desirable features, such as aligned nanotubes, good wetting, strong metal-BNNT interfaces through chemical reactions, and homogeneous dispersion of nanotubes in the matrix. BNNTs survived the high temperature and/or extreme pressure conditions involved in these processes. The chosen manufacturing approaches provided the opportunity to unravel a vast processing space, such as state of metal (solid versus liquid), the timescale of interactions $\left(10^{-3}\right.$ to $\left.10^{+3} \mathrm{~s}\right)$, range of temperatures $\left(10^{2}\right.$ to $\left.10^{3 \circ} \mathrm{C}\right)$ and processing pressures $\left(10^{-1}\right.$ to $\left.10^{+1} \mathrm{MPa}\right)$. 


\section{Key Findings}

\section{THRERMAL STABILITY}

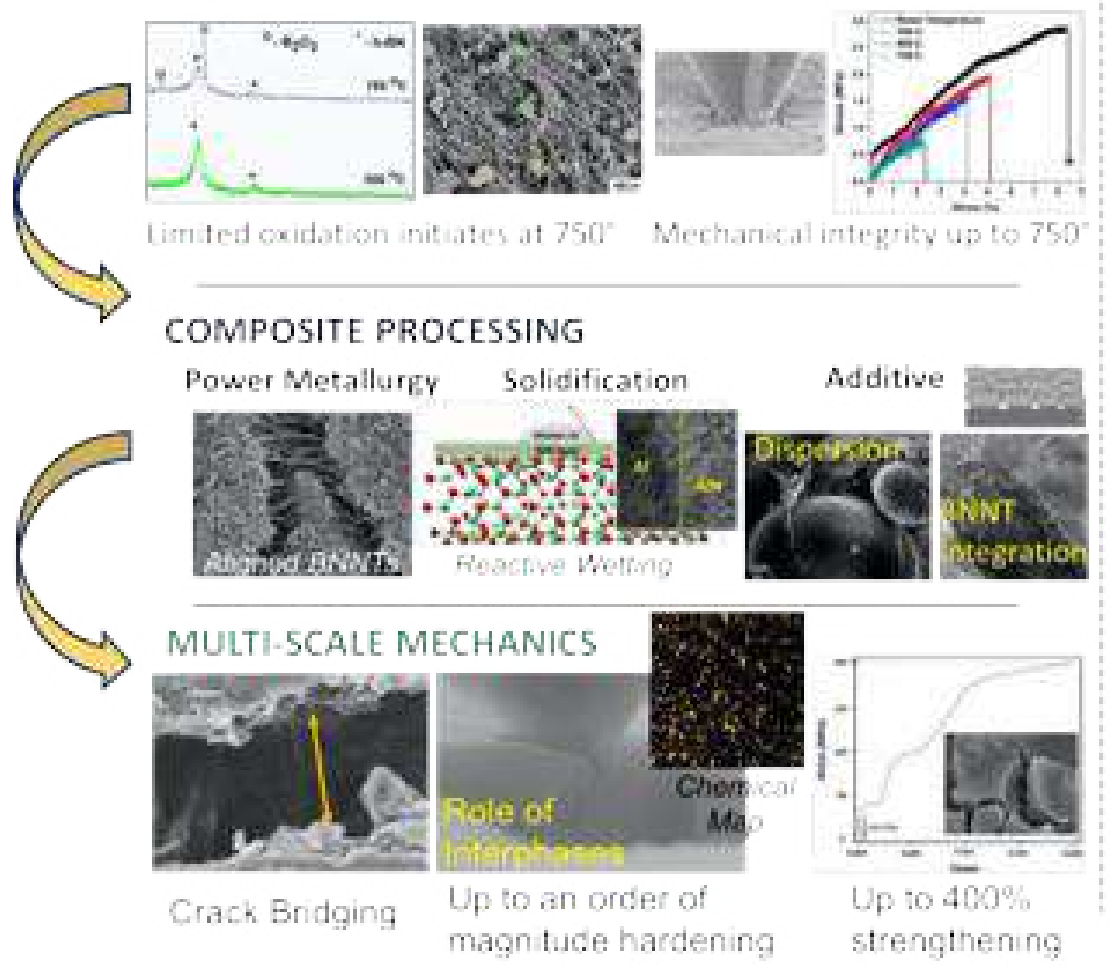

$\times$ Limitations

\& Possible Solutions

Non-Uniform

Themal Stability
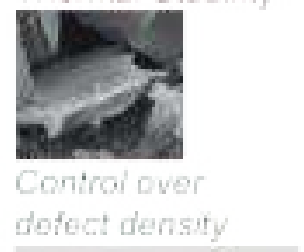

Local Bonding

Issues.

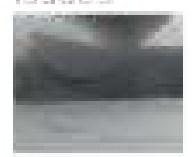

Post-grocessing

themat treatment

Arrested

Pasticity

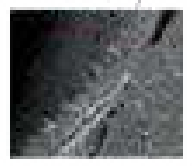

Dptimize BNivi

blume fraction

Figure 9.1. Schematic summarizing the focus areas of this dissertation and key conclusions derived from the investigations.

For developing insights into reinforcement mechanisms, in-situ investigation of deformation behavior was carried out in this work. Real-time and high-resolution electron microscopy revealed crack-bridging and crack-deflection by BNNTs. The role of interfacial reaction products was evaluated by combining mechanical studies with highresolution chemical mapping, using a variety of microscopic and spectroscopic analysis techniques. Interphases, in synergy with BNNT, improved the load-bearing ability of the metal matrices. Mechanical properties, such as tensile strength, elastic modulus, and 
hardness were characterized. Highly impressive improvements, in some cases by an order of magnitude, were recorded.

This research advances the fundamental understanding of microstructure evolution and mechanical response necessary to engineer BNNT-MMCs with desired and predictable mechanical properties (Figure 9.1). The subsequent sections summarize the findings of this research in detail. The lingering challenges and limitations are highlighted for each of the sections. The chapter ends with a future outlook for BNNT-MMCs, listing opportunities that lie ahead and recommending some exciting research directions that can be pursued. This dissertation unravels critical processing and mechanistic information for the effective utilization of this emerging class of structural composite.

\subsection{Chemical Stability and Structural Integrity of BNNT at Elevated Temperatures}

\subsubsection{Key Findings}

BNNT displayed physical and chemical stability up to temperatures exceeding $750^{\circ} \mathrm{C}$. Around $750^{\circ} \mathrm{C}$, some degree of oxidation and unzipping of nanotubes was observed. Microscopic and spectroscopic characterizations indicate the formation of 2D BN oxide platelets, which have an analogous structure to graphene oxide. However, not all the nanotubes underwent thermal transformations. In fact, a significant proportion of BNNTs was seen to retain their chemical and morphological make-up even up to temperatures as high as $1000^{\circ} \mathrm{C}$. A major challenge associated with the integration of nanofillers within 
metal matrices is their tendency to degrade or react under elevated processing temperatures. The fact that BNNT is stable at temperatures exceeding the melting point of lightweight structural metals, like $\mathrm{Al}$ and $\mathrm{Mg}$, makes it a promising choice for mechanical reinforcement.

Encouraged by its excellent thermal stability, we examined the load-bearing ability of BNNTs at elevated temperatures. The high-temperature mechanical properties of a freestanding BNNT buckypaper were probed by multi-scale in situ characterization techniques. High-resolution microscopy, in conjunction with digital image correlation analysis provided insights into temperature-dependent mechanisms in a BNNT macro-assembly. The buckypaper displayed excellent flexibility and resistance to failure for local compressive stresses as high as $60 \mathrm{MPa}$ even at $500^{\circ} \mathrm{C}$. The cyclic loading-unloadingreloading indentation of the buckypaper demonstrated excellent damage-tolerance over a long period of time as well as at elevated temperatures $\left(250\right.$ and $\left.500^{\circ} \mathrm{C}\right)$. Deformation for 50 cycles and local stresses up to $60 \mathrm{MPa}$ resulted in a minimal residual strain $\sim 1 \%$. The free-standing buckypaper is capable of bearing tensile stresses up to $750^{\circ} \mathrm{C}$, although the failure strength was found to drop from $\sim 14 \mathrm{MPa}$ at room temperature to $\sim 4 \mathrm{MPa}$ at $750^{\circ} \mathrm{C}$. BNNT buckypaper demonstrated viscoelastic response upon dynamic tensile loading. The loss tangent $(\tan \delta)$ was recorded to be as high as 0.5 , suggesting excellent energy dissipation/ impact-resistance ability. The viscoelastic characteristics were observed at elevated temperatures too (up to $750^{\circ} \mathrm{C}$ ). A wide range of service temperatures in which BNNT demonstrates structural integrity and load-bearing ability is exciting for hightemperature applications. 


\subsubsection{Considerations}

BNNT resists oxidation up to temperatures that are twice the degradation point for $\mathrm{CNT}^{7,8}$, establishing its edge for engineering advanced MMCs. Nevertheless, transformations seen around $750^{\circ} \mathrm{C}$ suggest an upper ceiling that needs to be considered while choosing processing temperatures. For instance, the selection of temperature for the casting of AlBNNT should preferably not exceed $700^{\circ} \mathrm{C}$. Integration of BNNTs in metals with a higher melting point, like Copper or Titanium, will be more complicated than lower melting point metals like Magnesium and Aluminum. Another point worthy of attention is that oxidation and transformations observed in the temperature range of 750 to $1000^{\circ} \mathrm{C}$ are not uniform, as noted above. A sizeable fraction of nanotubes can resist oxidative unzipping. The nonuniformity in thermal stability is believed to be due to Stone Wales defects in BNNTs, which are energetically favored sites for oxidation. The nanotubes that degrade earlier likely have higher defect concentration. Fourier transform infrared spectroscopy confirmed the presence of the homonuclear N-N and B-B defect sites used in this research project. This can have implications in MMC processing, as BNNTs may display different degrees of reactivity and stability, yielding a non-homogeneous microstructure.

\subsection{BNNT Alignment to Maximize Mechanical Strengthening of Aluminum}

\subsubsection{Key Findings}

Owing to its high aspect ratio, BNNT displays excellent mechanical strength along the axial direction. Therefore, an Al/BNNT/Al layered composite was fabricated by spark plasma sintering and cold rolling. Rolling of the composite resulted in perfectly aligned 
BNNTs along the deformation direction. A strong Al-BNNT interface was engineered by sputter-coating $\mathrm{Al}$ on BNNT mat prior to sandwiching. These long BNNTs survived the high temperature, high pressure, and severe plastic deformation conditions involved in SPS and cold rolling. Some key findings of the study are summarized below:

1. Highly impressive tensile strength ( $200 \mathrm{MPa})$ was obtained, with a $400 \%$ increase as compared to the standard value of strength for pure $\mathrm{Al}$ ( $40 \mathrm{MPa})$. Young's modulus was computed to be $134 \mathrm{GPa}$, which is double the standard modulus value for pure $\mathrm{Al}$ $(\sim 70 \mathrm{GPa})$.

2. The stress-strain curve exhibited three-stage yielding, associated with plastic deformation of the Al matrix, de-bonding of interfacial products, and failure of BNNTs.

3. Highly effective crack bridging was exhibited by directionally aligned long BNNTs.

4. Strengthening in the hybrid composite is believed to be occurring due to effective load transfer to directionally aligned ultra-long BNNTs. Formation of Al-BNNT clusters and reaction products ( $\mathrm{AlN}$ and $\mathrm{AlB}_{2}$ ) at the interface of $\mathrm{Al}$ and BNNT layer is a signature of strong interfacial interaction between the matrix and the secondary reinforcement.

\subsubsection{Limitations and Considerations}

While the composite fabricated by this approach demonstrated brilliant strength, the strainto-failure was rather low (2\%). This poses a challenge for applications requiring superior ductility. There are a few factors responsible for arrested ductility:

1. BNNT has an inherently low failure strain of less than $4 \%^{9}$. 
2. The formation of ceramic interphases can enhance the susceptibility to cracking at low tensile strains ${ }^{10}$.

3. Cold working of $\mathrm{Al}$ during the rolling step can cause high dislocation density in the matrix, inhibiting plasticity.

4. Poorly infiltrated Al-BNNT clusters can act as stress concentrators in the microstructure, and experience premature failure ${ }^{10}$.

Strategies such as heat treatment and tailoring of BNNT weight fraction can be helpful in raising the failure strain of the composite. These aspects have not been explored in the thesis.

\subsection{Integration of BNNT in Aluminum by Equilibrium Solidification}

\subsubsection{Key Findings}

Conventionally, casting has been at the center stage of the metallurgical processes due to its capability of fabricating complex shapes easily and economically. This work explored the feasibility of processing long boron nitride nanotube fibrils reinforced aluminum composites by the solidification route. BNNTs were found to survive high-temperature reactive conditions involved in the melting of $\mathrm{Al}$. AlN was obtained as the major interfacial product and was found to form a continuous film on the outer BNNT wall. AlN formation resulted in improved wetting of BNNT by molten Al. Calculations based on surface energies showed improved matrix-nanotube adherence due to AlN formation. Capillarity induced filling of BNNT by molten Al was also observed. The filling behavior is dependent on nanotube wetting which improves as Al-BNNT reaction progresses to form AlN. The 
sucked molten $\mathrm{Al}$ inside the nanotube was found to react with the BNNT wall to form AlN and a Boron-rich $\mathrm{AlB}_{10}$ phase, possibly due to the relative scarcity of $\mathrm{Al}$ inside the nanotube. The study demonstrated excellent wettability of BNNT by molten Al, supporting the possibility of developing high strength $\mathrm{BNNT}$ reinforced $\mathrm{Al}$ composites by casting route.

\subsubsection{Considerations}

While the formation of AlN at the interface is conducive to wetting, it is important to note that excess reactions can lead to compromised mechanical properties. Zhou et al. reported the formation of $\mathrm{Al}_{4} \mathrm{C}_{3}$ in $\mathrm{Al}-\mathrm{CNT}$ composite was helpful to promote tensile strength, but only up to $\sim 11 \%$ interphase content ${ }^{11}$. A similar effect is anticipated in Al-BNNT composites. Therefore, improved wetting due to AlN may not necessarily translate into brilliant mechanical properties. It is critical that the reactions are minimal and a sweet spot for maximum reinforcement is determined. Processing parameters, such as casting temperature and time, can be tweaked to tailor the fraction of interphases in the microstructure. These studies have not been undertaken in this dissertation, but merit a close evaluation in the future.

Another aspect worthy of attention is the capillary filling of BNNT by molten Al, which is interesting and provides an opportunity to increase the overall interfacial contact between the matrix and the nanotubes. However, the capillary effect is non-homogeneous in nature. Not all the nanotubes are filled with Al during processing, and different nanotubes are filled with metal to varying degrees. These microstructural variations can 
lead to non-uniform reinforcement efficacy. Variation in nanotube diameter and length is likely to be the most important factor responsible for inconsistent capillary filling. Raising the processing temperature can promote the filling action because the viscosity of molten $\mathrm{Al}$ goes down with temperature.

\subsection{Layer-by-Layer Build-up of Al-BNNT by Plasma Spray}

\subsubsection{Key Findings}

Additive manufacturing approaches have gained prominence due to the ease of fabricating near net-shaped structures. Therefore, plasma spray deposition of Al-BNNT composites was studied. Due to scalability and rapid processing, plasma spray is one of the most widely accepted solidification routes for developing metallic materials. A wet chemistry route was adopted to de-agglomerate BNNT clusters and obtain a uniformly dispersed mixture of metal particles and nanotubes. This feedstock was then plasma sprayed for layer-by-layer deposition of the metal matrix nanocomposite. BNNT survived the high temperature and reactive conditions in a plasma arc. Trace amounts of $\mathrm{AlB}_{2}$ and $\mathrm{AlN}$ were formed as the reaction products, which act as interfacial anchors in the microstructure due to covalent bond formation. Theoretical calculations revealed that rapid solidification in plasma spray enables the capture of BNNT in the molten Al by viscous drag mechanism. Effective wetting and capture of nanotubes by $\mathrm{Al}$ resulted in an order of magnitude improvement in nanohardness, attesting the suitability of BNNT as a reinforcement candidate using a nonequilibrium manufacturing technique. 


\subsubsection{Concerns and Considerations}

Plasma spray produces highly hierarchical microstructures, consisting of highly deformed powder particles solidified and bonded with each other. These particles are known as splats, which are the building blocks of the material. It has been reported in the past that the intersplat boundaries are susceptible to de-bonding and sliding under external mechanical

forces, a phenomenon known as Splat Sliding ${ }^{12}$. Therefore, the choice of processing parameters is important for ensuring effective inter-splat bonding. The addition of BNNT is likely to affect the bonding between the particles during spray. Keshri et al. reported improved splat-substrate bonding due to the addition of $\mathrm{CNT}$ to $\mathrm{Al}_{2} \mathrm{O}_{3}$. It is not obvious if this observation will also translate to a metallic coating. Furthermore, excess nanotubes or local clustering can deteriorate the bonding between metal particles. In the event of weak bonding, the composite is likely to fail along the splat interfaces and the inherent strength of BNNT won't be utilized to its full potential. The current work did not explore the available processing space to maximize bonding or to alter metal-nanotube interfaces. These processing considerations are important for engineering high-performance MMCs by plasma spray.

\subsection{Integrating BNNT in a Matrix with Low Ductility (AZ31 Magnesium Alloy)}

\subsubsection{Key Findings}

A major challenge with Mg alloys is their low ductility since they have only one closepacked plane (basal plane) and slip is primarily confined to this plane at room temperature. 
Adding a secondary filler (like nanotubes) can further lower the ductility because the filler phases tend to obstruct dislocation motion in the matrix. Therefore, we fabricated a multilayer sandwich-structured composite. In a sandwich composite, BNNT is not dispersed throughout the metal matrix, but it is incorporated in a layered fashion. It is noteworthy that Magnesium alloys have limited formability. Therefore, rolling, forging, or extruding them into desired shapes and forms is challenging. This inspired us to adopt a powder metallurgy (sintering) route. Sintering approach can be useful for near-net-shape manufacturing of composite components (with simple geometries) by designing dies in desired shape and dimensions. Another key consideration during the processing was the presence of native magnesium oxide in AZ31 powders. The oxide can hinder bonding between AZ31 and BNNT. Therefore, we chose a high-pressure sintering strategy (with an applied pressure of $400 \mathrm{MPa}$ ) to overcome the hindrance to bonding. High-pressure SPS proved to be an effective technique to engineer BNNT-reinforced Mg composites. Direct current-assisted consolidation allowed rapid sintering and bonding of AZ31 and BNNT. Interfacial reaction products $\left(\mathrm{Mg}_{3} \mathrm{~N}_{2}\right.$ and $\left.\mathrm{AlN}\right)$ were confirmed by TEM analysis, which proved to be helpful for effective stress-transfer between the matrix and the nanotubes. This was evidenced by the in-situ mechanical investigation, demonstrating crack-bridging due to BNNT in a sandwich-structured composite, providing superior failure-resistance. These findings are promising, and we envision BNNT to be a highly effective reinforcement candidate for augmenting the mechanical properties of lightweight magnesium. 


\subsubsection{Considerations}

The formation of interfacial products is helpful for BNNT-alloy adherence, but ceramic phases in the microstructure can compromise plasticity in an already low-ductility material system, like Mg. In fact, our attempts to roll the AZ31-BNNT pellet into composite sheets were unsuccessful. The composite fragmented into multiple pieces under rolling forces. Hot rolling or annealing of samples also failed to enhance the formability of the composite. This highlights a prominent plasticity issue that needs to be addressed. Some of the possible solutions can be:

1. Careful selection of alloy powder, to take into consideration grain sizes and phase make-up. While fine-grained powders provide strengthening, coarser-grained powders will display superior plasticity. It may be prudent to select a combination of powders for achieving a strength-ductility balance ${ }^{13}$.

2. SEM micrographs revealed a high density of BNNTs in the core of the sandwich composite. One of the classical approaches to resolve the issue can be reducing the filler content in the composite.

3. It may be useful to tailor the interphase content in the microstructure to balance strengthening with the retention of reasonable plasticity. This can be accomplished by careful selection of processing temperature and time. 


\subsection{In-situ Study of Mechanical Interactions between Matrix, Nanotubes and Interphases}

\subsubsection{Key Findings}

High-resolution, in-situ investigations were carried out to decipher the effect of BNNT addition as well as the formation of ceramic products in the Ti6Al4V matrix. Composites with two different processing temperatures were fabricated: a low temperature (LT) composite sintered at $750^{\circ} \mathrm{C}$ and a high temperature (HT) composite sintered at $950^{\circ} \mathrm{C}$. Mechanics of these composites were studied by indentation testing and real-time subsurface imaging by milling trenches in the regions of interest. Plastic flow, shear band propagation, cracking and crack-bridging were identified as the prominent mechanisms activated in the composite. Imaging along with testing allowed precise quantification of critical stresses and threshold energies required to activate these mechanisms. These calculations revealed plastic deformation mechanisms, such as shear band propagation and ductile cracking, are energetically favored to be activated first. The threshold specific energy expended to initiate each of the mechanisms are mentioned below (in increasing order):

- Ductile cracking: $6.5 \mathrm{GJ} \mathrm{m}^{-3}$

- Shear band propagation: $8 \mathrm{GJ}^{-3}$

- Particle de-bonding: $10.5 \mathrm{GJ} \mathrm{m}^{-3}$

- Transgranular cracking: $11.3 \mathrm{GJ}^{-3}$

- Inter-particle cracking: $19.5 \mathrm{GJ}^{-3}$ 
The effect of nanotubes and interphases was evaluated by calculating a reinforcement efficacy parameter $\left(\sigma_{c r} / H\right)$. The reason for choosing hardness (of pristine alloy) as a reference parameter is because of identical stress-states during hardness measurements and in-situ trench tests carried out in this work. Critical stresses required to initiate cracks in the composites were computed to be 1.3 to 1.8 times the hardness of the pristine alloy, which is encouraging.

Further, the degree of reactivity or the extent of interphases in the microstructure was computed by the XPS-spatial mapping of chemical species. An interface reaction index $\left(I_{R}\right)$, was defined to compare the area fraction of reaction products with respect to the original filler material (BNNT). This confirmed a rise in reactivity (by a factor of $\sim 1.4$ to 2.6) as the processing temperature was increased from 750 to $950^{\circ} \mathrm{C}$. This resulted in distinct deformation behavior in LT and HT composites: while the strengthening in LT composite was nanotube-dominated; the deformation behavior was interphase-dominated in HT composite.

\subsubsection{Concerns and Considerations}

Some of the particle interfaces were susceptible to interface cracking, de-bonding, and detachment. These regions in the microstructure are deeply concerning, as they can detach under stresses lower than the hardness of the pure alloy $\left(\sigma_{c r} / H<1\right)$. Poor bonding was primarily observed in LT composite, signifying $750^{\circ} \mathrm{C}$ is not sufficient for effective sintering. The role of time and pressure parameters requires further scrutiny to be able to consolidate the composites at lower temperatures. In addition to sintering issues, BNNT 
clustering in some regions of the microstructure was also responsible for catastrophic debonding under indentation loading. Excessive concentration of nanotubes at interfaces hinders effective particle-particle bonding. Therefore, there is a need to improvise the homogeneity of BNNT dispersion in the matrix.

Table 9.1. Summary of interfacial reaction products formed in three different metal matrices and three different processing approaches explored in this dissertation.

\begin{tabular}{lll}
\hline Composite System & Processing Approach & Interphases Formed \\
\hline Al-BNNT & Spark Plasma Sintering & $\mathrm{AlN}, \mathrm{AlB}_{2}$ \\
\hline Al-BNNT & Equilibrium Solidification & $\mathrm{AlN}, \mathrm{AlB}_{2}, \mathrm{AlB}_{10}$ \\
& (Casting) & \\
\hline Al-BNNT & Plasma Spray & $\mathrm{AlN} \mathrm{AlB}_{2}$ \\
\hline AZ31-BNNT & Spark Plasma Sintering & $\mathrm{Mg}_{3} \mathrm{~N}_{2}, \mathrm{AlN}$ \\
\hline Ti6Al4V-BNNT & Spark Plasma Sintering & $\mathrm{TiN}^{\mathrm{TiB}}, \mathrm{TiB}_{2}$ \\
\hline
\end{tabular}

\subsection{Future Directions}

\subsubsection{Interfacial Reactions vis-à-vis Mechanical Properties}

This dissertation does not delve into a precise correlation between the extent of interfacial chemical reactions and its effect on the load-bearing characteristics of BNNT-MMCs. Lahiri et al. ${ }^{14}$ and Bhuiyan et al. ${ }^{15}$ have explored the reactivity of BNNT with $\mathrm{Al}$ and Ti, respectively. These studies evaluated temperature and time effects on metal-BNNT 
reactivity. However, there is no parametric study correlating interphase(s) fraction with mechanical properties. Moreover, it has been noticed that there can be a combination of interphases formed during processing. For instance, this dissertation reported the formation of 2 or more reaction products in all the three metal matrices (summarized in Table 9.1). Some of the phases are expected to be stronger than the others. So far, the scientific studies reported in the literature have not delved into the mechanics of individual interphases and their effect on the load-bearing ability of BNNT-MMCs. Future efforts should be directed towards establishing this correlation to be able to engineer microstructures with desired mechanical characteristics.

\subsubsection{Exploration of Post-Processing Design Space}

This work primarily focused on exploring and exploiting processing parameters space to design BNNT-MMCs. However, there is tremendous scope to tailor composite properties by means of post-processing treatments, such as heat-treatment and mechanical working.

Xue et al. reported some degree of improvement in the tensile strength of Al-BNNT by heat-treatment ${ }^{16}$. This dissertation exploited the cold rolling technique to achieve BNNT alignment in the microstructure. However, there is a limited understanding of composite microstructure evolution and mechanical properties as a function of heat-treatment temperature or mechanical working forces. Thermal treatment holds promise to augment the ductility of the composites, if done correctly. Prolonged annealing is also likely to cause additional metal-BNNT reactions, an important consideration while selecting treatment parameters. The ability to tweak interphase fraction in the microstructure by heat-treatment will be a remarkable achievement for programming desired stress-transfer characteristics. 


\subsubsection{Mechanical Evaluation under Diverse Stress-States}

The current understanding of the mechanics of BNNT-MMCs is largely limited to tensile, compressive, and indentation response. This dissertation delved into in-situ characterization by indentation and double cantilever loading inside high-resolution microscopes for analyzing mechanisms in real-time. However, there is a multitude of unanswered questions pertaining to the deformation characteristics of BNNT-MMCs. Some of the important, yet unexplored mechanical properties are listed below:

- Flexural characterization

- Shear

- Impact-resistance

- Fatigue performance

- Wear or tribology

- Multiaxial deformation behavior

For a comprehensive assessment of BNNT's reinforcement potential, future mechanical investigations should look into these uncharted territories.

\subsubsection{Alternative Manufacturing Approaches}

One of the critical issues in MMC processing is nanomaterial agglomeration. The application of acoustic energy to disintegrate nanotube clusters and promote uniform dispersion has been studied for CNT and several ceramic nanoparticles. This technique, known as ultrasonic cavitation, can be promising to engineer BNNT-MMCs with 
homogeneous microstructures. The cavitation events during processing have been shown to break dendrites and promote globular microstructure, which is desirable for ductility. Therefore, the adoption of this technique can help offset plasticity challenges underscored in earlier sections.

There has been a great interest in the additive manufacturing of metals by selective laser sintering, electron beam melting, laser metal deposition, laser engineered net shaping and cold spray. There are no studies that report the fabrication of BNNT-MMCs by these techniques. Ultrashort processing times in additive manufacturing produces nonequilibrium solidification conditions, providing a limited window for controlling microstructure evolution and the integration of nanotubes. Detailed investigations will be required for developing process maps for effective wetting and capture of BNNT in metals using these manufacturing approaches.

\subsubsection{Multifunctional Properties}

In addition to exceptional mechanical properties, BNNT also displays radiation shielding ${ }^{17}$, high thermal conductivity ${ }^{18}$, and piezoelectric behavior ${ }^{19}$. These properties can be exploited to engineer multifunctional nanocomposites ${ }^{20}$. BNNTs can store hydrogen due to high surface area and high hydrogen binding energies ${ }^{17}$. This stored hydrogen can provide highly effective shielding against galactic cosmic radiation (GCR) because of its high charge-to-mass ratio. Considering the importance of thermally stable and lightweight composites in space applications, radiation shielding is of great interest. Future efforts 
should interrogate the effect of BNNT weight fraction, morphologies, and interfaces to achieve optimal radiation shielding by metal matrix composites.

\section{References}

1. Nautiyal, P. et al. Oxidative Unzipping and Transformation of High Aspect Ratio Boron Nitride Nanotubes into 'white Graphene Oxide' Platelets. Sci. Rep. 6, 29498 (2016).

2. Nautiyal, P., Zhang, C., Loganathan, A., Boesl, B. \& Agarwal, A. HighTemperature Mechanics of Boron Nitride Nanotube "Buckypaper" for Engineering Advanced Structural Materials. ACS Appl. Nano Mater. 2, 4402-4416 (2019).

3. Nautiyal, P. et al. Directionally Aligned Ultra-Long Boron Nitride Nanotube Induced Strengthening of Aluminum-Based Sandwich Composite. Adv. Eng. Mater. 18, 1747-1754 (2016).

4. Nautiyal, P., Gupta, A., Seal, S., Boesl, B. \& Agarwal, A. Reactive wetting and filling of boron nitride nanotubes by molten aluminum during equilibrium solidification. Acta Mater. 126, 124-131 (2017).

5. Nautiyal, P., Zhang, C., Boesl, B. \& Agarwal, A. Non-equilibrium wetting and capture of boron nitride nanotubes in molten aluminum during plasma spray. Scr. Mater. 151, 71-75 (2018).

6. Nautiyal, P. et al. Interface Engineering and Direct Observation of Strengthening Behavior in Field-Sintered Boron Nitride Nanotube - Magnesium Alloy Composite. Adv. Eng. Mater. 2000170, (2020).

7. Golberg, D., Bando, Y., Kurashima, K. \& Sato, T. Synthesis and characterization of ropes made of BN multiwalled nanotubes. Scr. Mater. 44, 1561-1565 (2001).

8. Chen, Y., Zou, J., Campbell, S. J. \& Caer, G. Le. Boron nitride nanotubes: Pronounced resistance to oxidation. Appl. Phys. Lett. 84, 2430-2432 (2004).

9. Wei, X., Wang, M.-S., Bando, Y. \& Golberg, D. Tensile tests on individual multiwalled boron nitride nanotubes. Adv. Mater. 22, 4895-4899 (2010).

10. Agarwal, A., Bakshi, S. R. \& Lahiri, D. Carbon Nanotubes: Reinforced Metal Matrix Composites. (CRC Press, Taylor \& Francis Group, 2011).

11. Zhou, W., Yamaguchi, T., Kikuchi, K., Nomura, N. \& Kawasaki, A. Effectively 
enhanced load transfer by interfacial reactions in multi-walled carbon nanotube reinforced Al matrix composites. Acta Mater. 125, 369-376 (2017).

12. Chen, Y., Bakshi, S. R., Agarwal, A. \& Street, W. F. Intersplat Friction Force and Splat Sliding in a Plasma-Sprayed Aluminum Alloy Coating during Nanoindentation and. ACS Appl. Mater. Interfaces 1, 235-238 (2009).

13. Luo, X., Huang, T., Wang, Y., Xin, Y. \& Wu, G. Strong and ductile AZ31 Mg alloy with a layered bimodal structure. Sci. Rep. 9, 5428 (2019).

14. Lahiri, D. et al. Insight into reactions and interface between boron nitride nanotube and aluminum. J. Mater. Res. 27, 2760-2770 (2012).

15. Bhuiyan, M. M. H., Li, L. H., Wang, J., Hodgson, P. \& Chen, Y. Interfacial reactions between titanium and boron nitride nanotubes. Scr. Mater. 127, 108-112 (2017).

16. Xue, Y. et al. Aluminum matrix composites reinforced with multi-walled boron nitride nanotubes fabricated by a high-pressure torsion technique. Mater. Des. 88, 451-460 (2015).

17. Thibeault, A. A. et al. Nanomaterials for radiation shielding. MRS Bulletin 40, 836-841 (2015).

18. Belkerk, B. E. et al. Thermal conductivity of vertically aligned boron nitride nanotubes. Appl. Phys. Express 9, 075002 (2016).

19. Bai, X. et al. Deformation-driven electrical transport of individual boron nitride nanotubes. Nano Lett. 7, 632-637 (2007).

20. Kang, J. H. et al. Multifunctional Electroactive Nanocomposites Based on Piezoelectric Boron Nitride Nanotubes. ACS Nano 9, 11942-11950 (2015). 


\section{APPENDIX}

\section{List of Videos Cited in the Thesis}

These real-time microscopy videos are an integral and important part of this dissertation. In-situ mechanical investigations were central to obtain critical insights into the deformation behavior of the nanocomposites engineered as a part of this research effort. The files are available for download.

\section{Chapter 5}

V 5.1: In-situ indentation of BNNT buckypaper at room temperature, inside SEM

V 5.2: In-situ indentation of BNNT buckypaper at $250^{\circ} \mathrm{C}$, inside SEM

V 5.3: In-situ indentation of BNNT buckypaper at $500^{\circ} \mathrm{C}$, inside SEM

V 5.4: In-situ cyclic indentation loading-unloading-reloading (50 cycles) of BNNT buckypaper at room temperature, inside SEM

V 5.5: In-situ cyclic indentation loading-unloading-reloading (50 cycles) of BNNT buckypaper at $250^{\circ} \mathrm{C}$, inside SEM

V 5.6: In-situ cyclic indentation loading-unloading-reloading (50 cycles) of BNNT buckypaper at $500^{\circ} \mathrm{C}$, inside SEM

V 5.7: High speed camera video of tensile failure of BNNT buckypaper at room temperature (captured at 5000 frames per second)

\section{Chapter 7}

V 7.1: BNNT anchors resisting crack opening in AZ31-BNNT (double cantilever testing)

V 7.2: Breaking of BNNT-AZ31 clusters before crack propagation in AZ31-BNNT (double cantilever testing)

\section{Chapter 8}

V 8.1: In-situ indentation of LT-Ti6Al4V-BNNT composite inside SEM, showing plastic flow

V 8.2: In-situ indentation of HT-Ti6Al4V-BNNT composite inside SEM, showing material pile-up 
V 8.3: In-situ indentation of HT-Ti6Al4V-BNNT composite inside SEM, showing microcracks around the indenter tip

V 8.4: In-situ indentation of LT-Ti6Al4V-BNNT composite inside SEM, showing interparticle crack propagation underneath the tip

V 8.5: In-situ indentation of LT-Ti6Al4V-BNNT composite inside SEM, showing particle detachment underneath the tip

V 8.6: In-situ indentation of LT-Ti6Al4V-BNNT composite inside SEM, showing particle sliding and liftoff underneath the probe

V 8.7: In-situ indentation of LT-Ti6Al4V-BNNT composite inside SEM, showing plastic flow of material underneath the tip

V 8.8: In-situ indentation of HT-Ti6Al4V-BNNT composite inside SEM, showing long range shear band propagation underneath the tip

V 8.9: In-situ indentation of HT-Ti6Al4V-BNNT composite inside SEM, showing crack initiation and propagation underneath the tip

V 8.10: In-situ indentation of HT-Ti6Al4V-BNNT composite inside SEM, showing ductile cracking underneath the indenter probe 
VITA

\section{PRANJAL NAUTIYAL}

2015-2020

Doctoral Candidate

Graduate Research Assistant and Presidential Fellow

Florida International University

Miami, Florida

2011-2015

B. Tech., PDPM Indian Institute of Information

Technology Design and Manufacturing Jabalpur

Madhya Pradesh, India

\section{SELECTED AWARDS AND HONORS}

1. Dissertation Year Fellowship, University Graduate School, Florida International University, Miami, Florida, 2020 (for 2 semesters).

2. Presidential Award, Outstanding Student Life Awards, Division of Academic and Student Affairs, Florida International University, Miami, Florida, April 2019.

3. TMS Best Paper Contest - Graduate Division - First Place, TMS Awards Ceremony, San Antonio, Texas, March 2019 (Award title: Real Time Observation of Splat Sliding in Cold Sprayed Metallic Coating by In-situ Mechanical Investigations).

4. Henry DeWitt Smith Scholarship, American Institute of Mining, Metallurgical, and Petroleum Engineers, TMS Awards Ceremony, San Antonio, Texas, March 2019.

5. Graduate Excellence in Materials Science (GEMS) Award, The American Ceramic Society - Basic Science Division, MS\&T Technical Meeting, Columbus, Ohio, October 2018.

6. TechConnect Defense Innovation Award, Defense TechConnect Summit, Tampa, Florida, October 2018 (Technology title: Boron Nitride Nanotube Based Advanced Metal Matrix Nanocomposites for Aerospace Applications).

7. First position, Poster Presentation (Engineering Category), Florida Statewide Graduate Student Research Symposium, University of South Florida, Tampa, Florida, April 2017 (Poster title: Unzipping of boron nitride nanotubes at elevated temperatures).

8. Presidential Fellowship, University Graduate School, Florida International University, Miami, Florida, 2015 (for 3 years).

\section{SELECTED JOURNAL PUBLICATIONS AND PRESENTATIONS}

P. Nautiyal, B. Boesl, A. Agarwal, In-situ Mechanics of Materials: Principles, Tools, Techniques and Applications, Springer International Publishing, ISBN 978-3-030-433192 (2020). 
1. P. Nautiyal, N. Denis, T. Dolmetsch, C. Zhang, B. Boesl, A. Agarwal, "Interface Engineering and Direct Observation of Strengthening Behavior in Field-Sintered Boron Nitride Nanotube-Magnesium Alloy Composite", Advanced Engineering Materials, In Press (2020).

2. P. Nautiyal, C. Zhang, V. K. Champagne, B. Boesl, A. Agarwal, "In-situ creep deformation of cold-sprayed aluminum splats at elevated temperatures", Surface \& Coatings Technology 372, 353-360 (2019).

3. P. Nautiyal, C. Zhang, B. Boesl, A. Agarwal, "Non-Equilibrium Wetting and Capture of Boron Nitride Nanotubes in Molten Aluminum During Plasma Spray", Scripta Materialia 151, 71 (2018).

4. P. Nautiyal, M. Mujawar, B. Boesl, A. Agarwal, "In-situ Mechanics of 3D Graphene Foam Based Ultra-stiff and Flexible Metallic Metamaterial”, Carbon 137, 502 (2018).

5. P. Nautiyal, C. Zhang, V. K. Champagne, B. Boesl, A. Agarwal, "In-situ Mechanical Investigation of the Deformation of Splat Interfaces in Cold-Sprayed Aluminum Alloy”, Materials Science \& Engineering A 737, 297 (2018).

6. P. Nautiyal, B. Boesl, A. Agarwal, "The Mechanics of Energy Dissipation in a ThreeDimensional Graphene Foam with Macroporous Architecture", Carbon 132, 59 (2018).

7. P. Nautiyal, B. Boesl, A. Agarwal, "Harnessing three dimensional anatomy of graphene foam to induce superior damping in hierarchical polyimide nanostructures", Small 13, 1603473 (2017).

8. P. Nautiyal, A. Gupta, S. Seal, B. Boesl, A. Agarwal, "Reactive wetting and filling of boron nitride nanotubes by molten aluminum during equilibrium solidification", Acta Materialia 126, 124 (2017).

9. P. Nautiyal, A. Loganathan, R. Agrawal, B. Boesl, C. Wang, A. Agarwal, "Oxidative unzipping and transformation of high aspect ratio boron nitride nanotubes into white graphene oxide platelets", Scientific Reports 6, 29498 (2016).

10. P. Nautiyal, C. Rudolf, A. Loganathan, C. Zhang, B. Boesl, A. Agarwal, "Directionally aligned ultra-long boron nitride nanotube induced strengthening of aluminum-based sandwich composite", Advanced Engineering Materials 18, 1747 (2016).

11. Real Time Imaging of Deformation Mechanisms in Boron Nitride Nanotube-Metal Matrix Composites at Multiple Length Scales, TMS Annual Meeting \& Exhibition, San Diego, California, USA (February 2020).

12. Scalable Manufacturing of Boron Nitride Nanotube Based Metal Matrix Composites, 14th International Ceramics Congress, Perugia, Umbria, Italy (June 2018).

13. In situ Mechanical Investigation of Splat Sliding in Cold Sprayed 6061 Al Coatings, International Thermal Spray Conference 2018, Orlando, Florida, USA (May 2018). 